\title{
PRODUCTION CORRELATIONS OF HADRONICALLY PRODUCED CHARMED PARTICLES
}

\author{
A DISSERTATION \\ SIBMITTED TO THE DEPARTMENT OF PHYSICS \\ AND THE COMMITTEE ON GRADUATE STUDIES \\ of C'arnegie Mellon University \\ IN PARTIAL FULFILLMENT OF THE REQUIREMENTS \\ POR THE DEGREE OF \\ DOCTOR OF PHILOSOPHY
}

$\mathrm{By}$

Anne P. Freyberger

August. 1990

FERMILAB LIBRARY 


\title{
Production Correlations of Hadronically Produced Charmed Particles
}

\begin{abstract}
.
Charm pair production results are presented on the 37 charm pairs collected during the 1985 run of experiment E653 at Fermilab. The pair differential cross section is fitted the functional form $\frac{d \sigma}{d X_{f} d P_{t}^{2}} \sim\left(1-\left|X_{f}\right|\right)^{n} e^{-b P_{t}^{2}}$. The maximum likelihood fit yields $n=5.0 \pm 1.5$ and $b=0.65_{-0.08}^{+0.10}(\mathrm{GeV} / \mathrm{c})^{-2}$. The charm pair mass distribution is fitted to an exponential, $\mathrm{e}^{-a N S(D D)}$, with $a=0.75 \pm 0.15\left(\mathrm{GeV} / \mathrm{c}^{2}\right)^{-1}$. The charm pair $\mathrm{X}_{f}, \mathrm{P}_{t}^{2}$, rapidity gap, mass and azimuthal opening angle distributions are presented and compared with distributions from previously published results. The azimuthal opening angle and rapidity gap of the charm pair exhibit correlations when compared to charm pairs produced uncorrelated by a Monte Carlo. The $\mathrm{P}_{t}^{2}$ distribution has an excess of events at $P_{t}^{2}>3.0(\mathrm{GeV} / \mathrm{c})^{2}$. Comparisons are made to previously published results on Drell-Yan di-lepton production. The data slow similarities to the di-lepton results in the pair $\left\langle\mathrm{P}_{t}^{2}\right\rangle$ and pair mass distributions. The analysis method is described in detail, including the development and performance of a momentum estimator used for the charm parent momentum.
\end{abstract}


In memory of Mom and Dad... 


\section{Contents}

1 Introduction $\quad 1$

2 Hadro-Production of Charm: A Review 5

2.1 Introduction ........................ 5

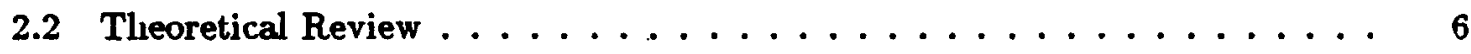

2.2.1 Total Charm Cross Section . . . . . . . . . . . . . 6

2.2.2 Charm Correlations $\ldots \ldots \ldots \ldots \ldots$. . . . . . . .

2.3 Hadronic Charm Production Results to Date . . . . . . . . . . 10

2.3.1 Total Charm Cross Section ... . . . . . . . . . . 12

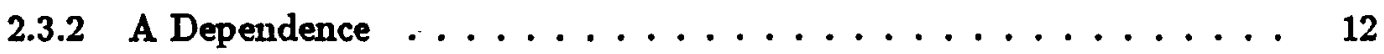

2.3.3 Differential Cross Section .................. 13

2.3.4 Charm Pair Results . . . . . . . . . . . . . . . . 15

2.4 Drell-Yan Di-Lepton Production . . . . . . . . . . . . 20

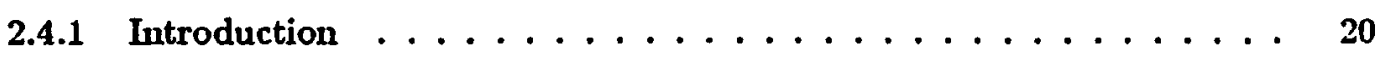

2.4.2 The Drell-Yan-Charm connection .............. 20 
2.4 .3 Drell-Yan Scaling ... . . . . . . . . . . . . . 22

2.4 .4 Structure Functions $\ldots \ldots \ldots \ldots \ldots \ldots$

3 The Hardware $\quad 26$

3.1 Introduction ...................... 26

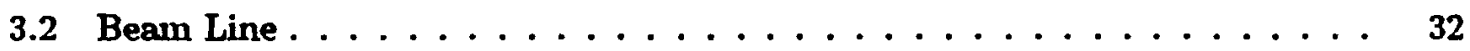

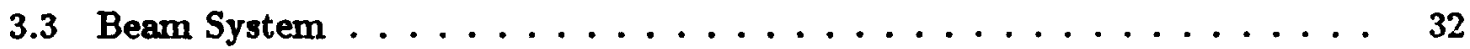

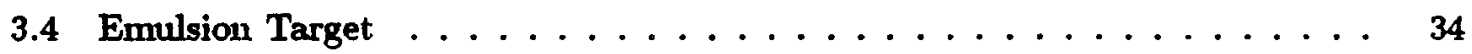

3.4.1 Vertical Emulsion Module .................. 37

3.4.2 Horizontal Emulsion Module $\ldots \ldots \ldots \ldots$. . . . . . . . . 37

3.4.3 Emulsion Alignment ..................... 40

3.5 Charged Particle Spectrometer . . . . . . . . . . . . . . . 41

3.5.1 Vertex Silicon Strip Detectors (VSSD's) . . . . . . . . . . . 41

3.5.2 Analyzing Magnet . . . . . . . . . . . . . . . . . 42

3.5.3 Spectrometer Drift Chambers . . . . . . . . . . . . . 46

3.5.4 Momentum ....................... 49

3.6 Muon System . . . . . . . . . . . . . . . . . . . . 49

3.6.1 Muon Spectrometer .................... 51

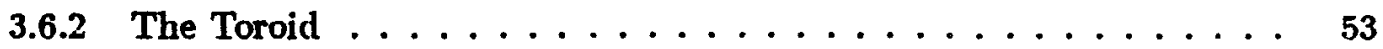

3.6.3 Muon Hodoscope . . . . . . . . . . . . . . . . . 54

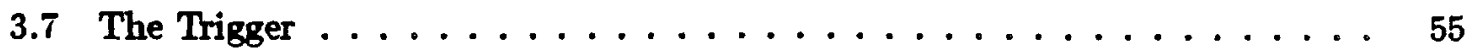

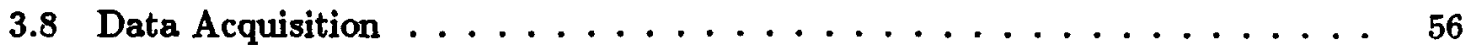


3.8.1 Fastbus Hardware .................... 59

3.9 The 1985 Run and Overall Performance ................ 61

4 Charm Selection $\quad 65$

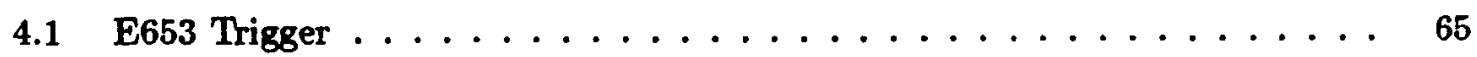

4.2 Event Reconstruction and Emulsion Predictions . . . . . . . . . 66

4.3 Emulsion Scanning . . . . . . . . . . . . . . . . 69

4.3.1 Automated Scanning . . . . . . . . . . . . . . 70

4.3.2 Graphic Analysis . . . . . . . . . . . . . . . 72

4.3.3 Decay Search ...................... 72

4.3.4 Interaction Background ................. 73

4.4 Emulsion Charm Selection . . . . . . . . . . . . . 73

4.4 .1 Decay Topologies ...................... 74

4.5 Refitting ............................. 75

4.6 Final Charm Pair Sample . . . . . . . . . . . . . . 76

4.6.1 Level 1: A Muonic Vertex ................... 77

4.6.2 Level 2: Two or more decay vertices . . . . . . . . . . . 77

4.6.3 Level 3: Complete tracking . . . . . . . . . . . . 78

4.6.4 Level 4: $\mathbf{M}_{\min }$ Cuts $\ldots \ldots \ldots \ldots \ldots \ldots$

4.6.5 Level 5: Multiprong-Multiprong Charm pairs . . . . . . . . . 84

$\begin{array}{llr}5 & \text { Backgrounds } & 87\end{array}$

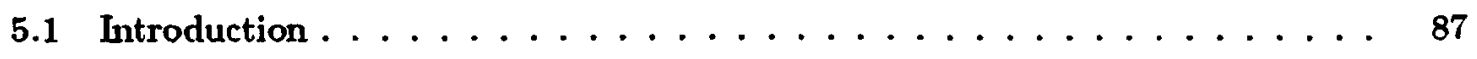


5.2 Diffractive Background ..................... 88

5.3 Wrong Sign Charged Background . . . . . . . . . . . . . . 90

5.3.1 Hadronic Kinks (C-1's) . . . . . . . . . . . . . 90

5.3.2 Hadronic Tridents $(\mathrm{C}-3$ 's $) \ldots \ldots \ldots \ldots . \ldots \ldots$

5.3.3 Muonic Tridents $(\mathrm{C}-3 \mu \mathrm{s} s) \ldots \ldots \ldots \ldots$

5.4 Wrong charge vertices $\ldots \ldots \ldots \ldots \ldots \ldots$

5.5 Final Background Determination . . . . . . . . . . . . . 97

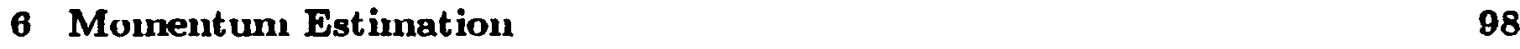

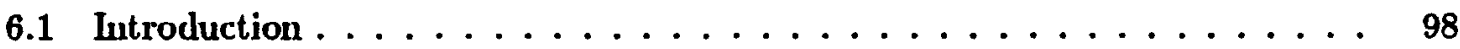

6.2 Kinematics . . . . . . . . . . . . . . . . . . . . . . 99

6.2.1 Mass Assignments . . . . . . . . . . . . . . . . 99

6.3 The Resolution Function and Monte Carlo Studies . . . . . . . . . . . 103

6.4 The $0-C$ Analogy . . . . . . . . . . . . . . . . 106

6.5 Other Charm Decay Modes . . . . . . . . . . . . . . . . 108

7 Maximum Likelihood Technique and Efficiencies 119

T.1 Introduction . . . . . . . . . . . . . . . . . . 119

7.2 Probabilistic Motivation . . . . . . . . . . . . . . 120

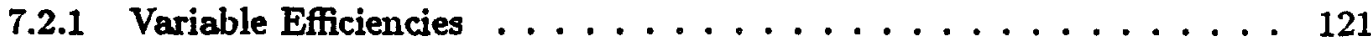

7.2.2 Resolution Functions . . . . . . . . . . . . . . . . 121

7.2.3 Maximum Likelihood Implementation in E653 . . . . . . . . . 123

7.3 Topological Efficiencies . . . . . . . . . . . . . . . . 123 
7.3.1 Single Charm Efficiencies . . . . . . . . . . . . . 124

7.4 Charm Pair Efficiencies $\ldots \ldots \ldots \ldots \ldots \ldots \ldots \ldots$

7.5 Validity of the Maximum Likelihood Technique . . . . . . . . . . . 132

8 Charm Pair Production and Correlation Results 136

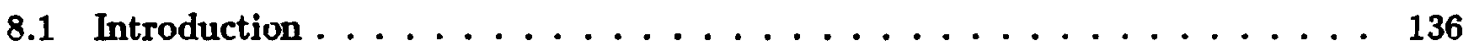

8.2 Charm Pair Monte Carlo . . . . . . . . . . . . . . 137

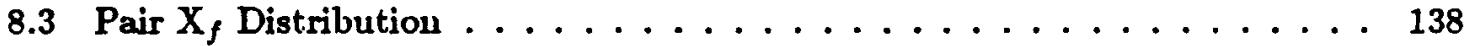

8.4 Pair $\mathrm{P}_{t}^{2}$ Distribution . . . . . . . . . . . . . . . 140

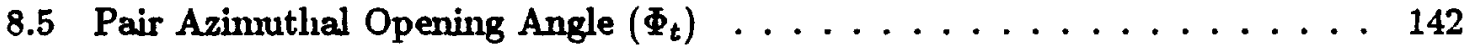

8.6 Charn Pair Mass . . . . . . . . . . . . . . . . . . . . . . 142

8.7 Chann Pair Rapidity Gap $(\Delta \mathrm{Y}) \ldots \ldots \ldots \ldots$

8.8 Comparison to previous results . . . . . . . . . . . . . 145

$\begin{array}{llr}\theta & \text { Conclusions } & 149\end{array}$

A The Lund Monte Carlo

$\begin{array}{lr}\text { B Event listings } & \mathbf{1 6 0}\end{array}$ 


\section{List of Tables}

1 List of fixed target charm experiments with hadron beams. . . . . . . . 11

$2 \quad$ Measured values for $\alpha$ from different fixed target experiments. . . . . . . 13

3 References for the E653 spectrometer and its components. . . . . . . 31

4 Chemical composition of Fuji ET-7B nuclear emulsion. The value for $\langle A\rangle$ was calculated assuming a linear dependence on A. . . . . . . . 36

5 The individual detector resolutions for the E653 spectrometer. . . . . . . . 64

6 Listing of the four different classes of cuts used in selecting events for emulsion scanning. ........................... 69

7 Typical emulsion scanning predictions for a vertical emulsion module. . . 70

8 Event through put for the different emulsion scanning stages. . . . . . . . 73

9 Allowed range for $M_{\pi \pi}$ of neutral V-2 decays. . . . . . . . . . 84

$10 M_{\min }$ cut values for the different topologies. The sigma is the measured error on the $M_{\min }$ for the decay. $\ldots \ldots \ldots \ldots \ldots$

11 Succession of cuts appli t to the charm candidate sample. . . . . . . 85

12 Emulsion-emulsion charm pair topologies in the final charm pair sample. . 86 
13 Eunulsion-counter charm pair topologies in the final charm pair sample. . . 86

14 The number of non-diffractive vertices with all light shower tracks match to the spectrometer, scaled to yield an estimate of the diffractive background in the complete data set. $\ldots \ldots \ldots \ldots \ldots \ldots$

15 Diffractive background estimates in the complete data set and the charm pair data set at the last two stages of charm pair selection. . . . . . . . . 89

16 Sign correlations in hadronic kinks with respect to the $\mu$ in the event. Right sign kinks had the opposite sign of the muon sign in the event. . . . . . . . 94

17 Sign correlation for hadrowic tridents (C-3's) with respect to the $\mu$ in the event. Right sign tridents have the opposite charge sum of the muon charge

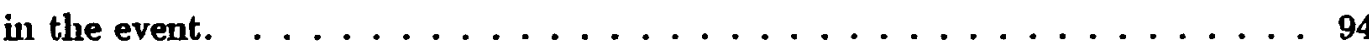

18 Sign correlations for muonic tridents. Right sign means the sign of the muon equals the charge sum of the vertex. . . . . . . . . . . 95

19 Wrong charge vertices in the complete data set. $\ldots \ldots \ldots \ldots$

20 Wrong charge vertices in the Level 4 charm pairs. . . . . . . . . . . . 96

21 Wrong charge vertices in the Level 5 charm pairs. . . . . . . . . . . 96

22 Total Background for the charm pairs. The diffractive interaction background is lower than measured due to additional emulsion measurements. . . . . . . 97

23 Branching Ratios used in the Monte Carlo development of the momentum estimator and charm selection. . . . . . . . . . . . . . . . . . 104

24 Comparison of weighted average values measured in two previous charm pair experiments and the values presented in this thesis. $\ldots \ldots \ldots \ldots \ldots$. . . 147 
25 Event listings for the 37 charm pairs. . . . . . . . . . . . . 163

26 Event listings for the 37 charm pairs continued from the previous page. . . 164

27 Event listings for the 37 charn pairs continued from the previous page. . . 165

28 Event listings for the 37 charm pairs continued from the previous page. . . 166 


\section{List of Figures}

1 The quark (left) and lepton (right) families and their measured masses ${ }^{1}$. . 2

2 Leading order parton diagrams for heavy quark production. . . . . . . 7

3 Next to leading order parton diagrams for heavy quark production. . . . 8

$4 \mathrm{~K}$-factor for the total charm cross section as a function of beam energy. . . 9

5 Total charm cross section measurements from different fixed target charm experiments as a function of center of mass energy. $\ldots \ldots \ldots \ldots$

6 The measured values for the exponent $n$ for the different fixed target exper-

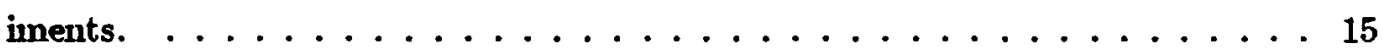

7 The measured values for the $\left\langle P_{t}^{2}\right\rangle$ from the different fixed target experi-

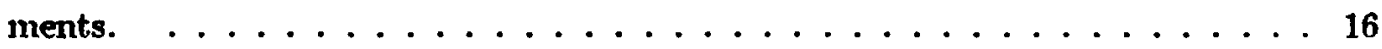

8 The $\Phi_{t}$ distribution measured by LEBC-PP. There are 107 geometrically reconstructed pairs in the plot. $\ldots \ldots \ldots \ldots \ldots$

9 The weighted $\mathrm{X}_{f}$, mass, $\mathrm{P}_{t}^{2}$ and $\Delta \mathrm{Y}$ distributions for the 17 LEBC-PP charm pairs. The dashed curve is from the LUND MC with a $\delta$ fri-mentation function and the solid curve is the LUND MC with LUND fragmentation. $\quad 19$ 
10 Leading order production diagram for di-lepton production in hadronic in-

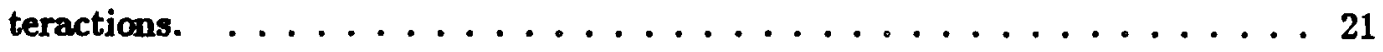

11 Plan view of the compact E653 spectrometer. . . . . . . . . . . 28

12 The E653 coordinate system. . . . . . . . . . . . . . . . . 29

13 The Fermilab accelerator complex with the fix target beam lines. . . . . . 33

14 The integrated beam spot of the $800 \mathrm{GeV}$ proton beam measured by the

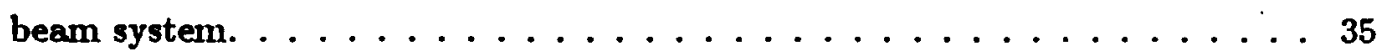

15 The configuration of a vertical emulsion module. . . . . . . . . 38

16 Horizontal emulsion target module configuration. . . . . . . . . . . 39

17 Read out pattern for the vertex silicon strip detectors. . . . . . . . . 43

18 Residuals in the vertex silicon strip detectors. . . . . . . . . . . . 44

19 The poles of the analyzing magnet, SCM-104. . . . . . . . . . 45

20 Wiring diagram for the fine and coarse cells in the spectrometer drift cham-

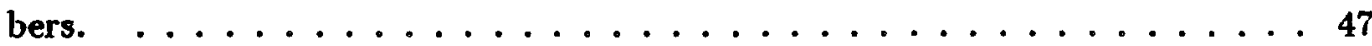

21 Position residuals for the spectrometer drift chambers. . . . . . . . . 48

22 Plan view of the muon system. . . . . . . . . . . . . 50

23 Position residuals for four of the cells in the muon drift chambers. . . . . 52

24 The beam and interactions counters of the E653 trigger system. . . . . . 57

25 Logical schematic of the E653 trigger. . . . . . . . . . . . 58

26 The event data path from the the FASTBUS crates to the magnetic tape. 62

27 The integrated number of data triggers written to tape during the 1985 run

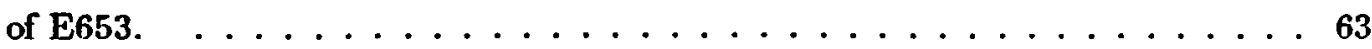


28 Cut variables applied the muon track to select events for emulsion scanning.

29 Impact paramter for tracks coming from the primary vertex. . . . . . . 71

$30 M_{\min }$ distributions for muonic decays of $D^{\circ}$ and $D^{+}$mesons. The solid curve is for Monte Carlo and the dashed represents the data before any cuts. The arrows point to the upper and lower bounds on $M_{\min }$ required for a decay to be considered chann. . . . . . . . . . . . . . . . 80

$31 M_{m i n}$ distributions for non-muonic decays of $D^{\circ}$ and $D^{+}$mesons. The solid curve is for Monte Carlo and the dashed represents the data before any cuts. The arrows point to the upper and lower bounds on $M_{\min }$ required for a decay to be considered charm. . . . . . . . . . . . . 81

32 Wrong sign $\mu$ events used to estimate cliarge background. . . . . . . . . . 91

$33 \quad P_{t}$ distribution for right sign C-1's found in the emulsion. $\ldots \ldots \ldots$

$34 P_{t}$ distribution for wrong sign C-1's found in the emulsion. . . . . . . 93

35 Momentum vectors in the laboratory frame for a neutral decay. . . . . . . 100

36 Momentum vectors in the center-of-mass (COM) frame for a neutral decay. Note that the ouly momentum in the center-of-mass frame in perpendicular to the parent direction in the LAB frame. . . . . . . . . . . 100

37 Mass assignments for decays with a tagged muon. . . . . . . . . . . . 101

38 Mass assignments for decays without a tagged muon. . . . . . . . . . . . . 102

39 The momentum solutions for the $0-\mathrm{C}$ equation as a function of parent mass. 108

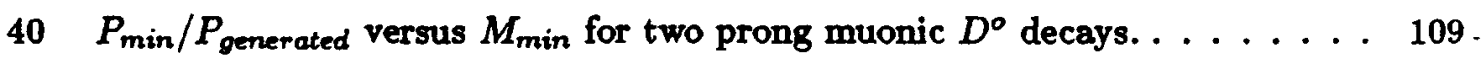




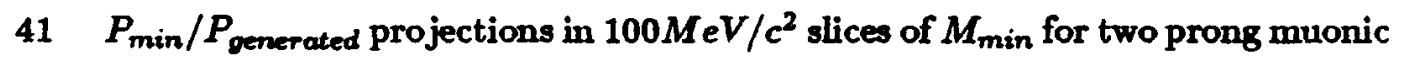

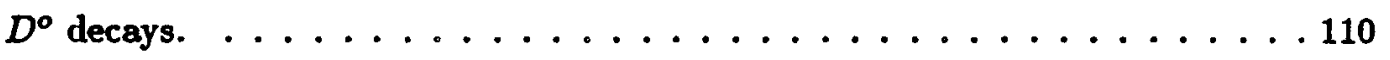

$42 P_{\min } / P_{\text {generated }}$ versus $M_{\min }$ for two prong hadronic $D^{\circ}$ decays. . . . . 111

$43 P_{\min } / P_{\text {generated }}$ projections $100 \mathrm{MeV} / \mathrm{c}^{2}$ slices of $M_{\min }$ for two prong hadronic

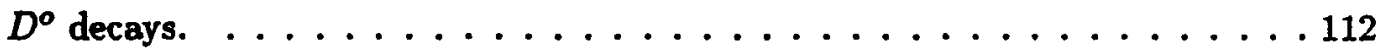

$44 P_{\min } / P_{\text {generated }}$ versus $M_{\min }$ for three prong muonic $D^{+}$decays. . . . . 113

$45 P_{\min } / P_{\text {generated }}$ projections in $100 \mathrm{MeV} / \mathrm{c}^{2}$ slices of $M_{\min }$ for three prong muonic $D^{+}$decays. . . . . . . . . . . . . . . . . 114

$46 P_{\min } / P_{\text {penerated }}$ versus $M_{\min }$ for three prong hadronic $D^{+}$decays. . . . . 115

$47 P_{\min } / P_{\text {generated }}$ projections in $100 \mathrm{MeV} / \mathrm{c}^{2}$ slices of $M_{\min }$ for three prong hadronic $D^{+}$decays. ......................... 116

$48 P_{\min } / P_{\text {penerated }}$ versus $M_{\min }$ for four prong hadronic $D^{\circ}$ decays. . . . . 117

$49 P_{\min } / P_{\text {generated }}$ projections in $100 \mathrm{MeV} / \mathrm{c}^{2}$ slices of $M_{\min }$ for four prong hadronic $D^{\circ}$ decays. . . . . . . . . . . . . . . . 118

50 Generic resolution function $R\left(x_{i}, x_{g}\right)$ as function of $x_{g} \ldots \ldots \ldots$

$51 X_{f}$ efficiencies for muonic decays in the emulsion block. . . . . . . 126

$52 X_{f}$ efficiencies for hadronic decays in the emulsion block. . . . . . . . 12t

$53 \quad P_{t}^{2}$ efficiencies for muonic decays in the emulsion block. . . . . . . . 128

$54 P_{t}^{2}$ efficiencies for hadronic decays in the emulsion block. . . . . . . . 129

55 Average efficiency as a function of $X_{f}$ for the 37 charm pairs. . . . . . 130

56 Average efficiency as a function of $\mathrm{P}_{t}^{2}$ for the 37 charm pairs. . . . . . 131

57 Average efficiency distribution as a function of charm pair mass. . . . . . 133 
58 Average efficiency distribution as a function of charm pair rapidity gap. . . 133

59 Comparison of the input Monte Carlo $n$ value with that returned by the maximum likelihood method for charm pairs. . . . . . . . . . 135

60 Comparison of the input Monte Carlo $b$ value with that returned by the maximum likelihood method for charm pairs. . . . . . . . . . . 135

61 The $\mathrm{X}_{f}$ distribution for the 37 charn pairs. The solid line is the result of a maximum likelihood fit to $\left(1-\mathrm{X}_{f}\right)^{n}$. The dashed histogram is from $\mathrm{MC} \Phi_{t}$ correlated charm pairs. . . . . . . . . . . . . . . . . . 139

62 The weighted $\mathrm{P}_{t}^{2}$ distribution for the charm pairs. The solid line is the result of a maximum likelihood fit to $e^{-b P_{t}^{2}}$. The dashed histogram is for $\Phi_{t}$ correlated charm pairs. . . . . . . . . . . . . . . . 141

63 The $\left\langle\mathrm{P}_{t}^{2}\right\rangle$ versus $\mathrm{s}$ for E653 and LEBC-PP charm pairs. The curve is a least squares fit to $\left\langle\mathrm{P}_{t}^{2}\right\rangle=\mathbf{k}_{t}^{2}+\mathbf{s} \cdot$ constant. $\ldots \ldots \ldots \ldots \ldots$

$64 \Phi_{t}$ (Azimuthal opening angle of the charm pair) distribution for the 37 charm

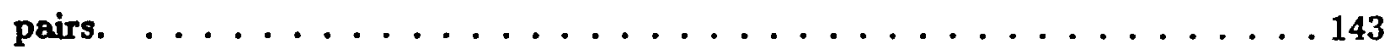

65 Charm pair mass distribution. The solid line is the result of a fit to $e^{-a M}$. The dotted histogram is the mass distribution obtained from the $\Phi_{t}$ correlated

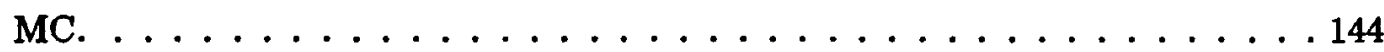

66 The rapidity gap distribution of the charm pairs. The solid histogram is for the data. The dashed histogram is for $\Phi_{t}$ correlated MC charm pairs. . . . 146

67 The $\Phi_{t}$ distribution for the 37 charm pairs (solid) and the LUND MC (dots). 155 
68 The weighted $\mathrm{X}_{f}$ distribution for the 37 charm pairs (solid) and the LUND

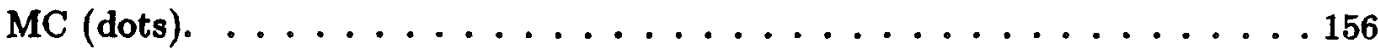

69 The weighted charm pair mass distribution. Data (solid). LUND MC (dots). 157

70 The weighted rapidity gap distribution for the 37 charm pairs (solid) and that obtained from the LUND MC (dots). . . . . . . . . . 158

71 The weighted $\mathrm{P}_{t}^{2}$ distribution for the 37 charm pairs (solid) and the LUND MC charm pairs (dots) $\ldots \ldots \ldots \ldots \ldots \ldots$ 


\section{Chapter 1}

\section{Introduction}

The last decade has seen some remarkable achievements in particle physics. The discovery of the intermediate vector boson, $W^{ \pm}[1,2]$, in 1983 unified the electromagnetic and weak forces. This unification continues the tradition of representing what appear to be different interactions by one encompassing interaction. The subsequent discovery of the $Z^{\circ}$, partner to the $W^{ \pm}$, and the detailed study of its mass and width have restricted the number of lepton families to three $[3,4,6,7]$.

The constituents within the standard model are divided into two distinct groups. Figure 1 depicts the three generations of quarks and leptons. Lepton masses and limits are from [38], $d, u, s, c$ and $b$ quark masses are from [5] and the $t$ quark mass limit is from [8]. The $\mathrm{Z}^{\circ}$ mass and width only limit the lepton sector. However, symmetry suggests that the numbers of lepton and quark generations are the same. The discovery of the tau neutrino and top quark would complete the constituents needed within the standard model framework. Exhaustive searches at $p \bar{p}$ colliders have yielded no clear evidence for the top quark. 


$$
\left(\begin{array}{ccc}
u & c & t \\
(\sim 0.350) & (\sim 1.5) & (>77) \\
d & s & b \\
(\sim 0.350) & (\sim 0.55) & (\sim 4.5)
\end{array}\right)\left(\begin{array}{ccc}
e & \mu & \tau \\
(0.000511) & (0.1056) & (1.870) \\
& & \\
\nu_{e} & \nu_{\mu} & \nu_{\tau} \\
(<18 e v) & (<0.25 \mathrm{MeV}) & (<35 \mathrm{MeV})
\end{array}\right)
$$

Figure 1: The quark (left) and lepton (right) families and their measured masses ${ }^{1}$.

If it exists its mass must be greater than $77 \mathrm{GeV} / \mathrm{c}^{2}[8]$.

The quarks interact via the strong, electroweak and gravitational forces. The leptons interact via the electroweak and gravitational forces. The strong force is described by the theory of quantum chromodynamics (QCD). QCD is an extension of the highly successful theory of the electromagnetic force, quantum electrodynamics (QED), to the strong force. The electric field of QED is analogous to the color field of QCD, with the photon mediator of QED replaced by the mediator of the strong force, the gluon. QED has been tested to less than one part per billion by studying the electron magnetic moment. QCD has not undergone such strenuous testing. One of the processes described by QCD is heavy quark (charm, bottom or top) production in hadronic interactions.

The study of charm production provides a test of QCD and aids in understanding bottom and top production within the QCD framework. QCD calculations of charm production have been restricted by the questionable validity of the perturbation expansion of the calculation. Experimental results of charm production provide crucial information linking the

\footnotetext{
${ }^{1}$ Charge conjugate particles are implied throughout this thesis, unless stated otherwise.
} 
perturbative regime of $\mathrm{QCD}$ to the non-perturbative regime.

As the search for the top quark intensifies, the understanding of heavy quark production becomes important. Charm and bottom decays contribute background leptons to the top signal and this background must be accurately subtracted in order to reveal new signals. Knowledge of the procluction characteristics, kinematic and dynamic, of the top quark will aid in its discovery.

Fermulab experment E653 was proposed in 1981 [63] to study the production and decay properties of particles containing heavy quarks $(c, b)$. E653 used an $800 \mathrm{GeV}$ proton beam. incident on an emulsion target followed by 18 planes of $50 \mu$ pitch silicon strip detectors. This unique combination of a ligh resolution electronic spectrometer and the even higher resolution of the target emulsion provided E653 with a large acceptance for charm and beauty pairs. The visual technique of the emulsion allowed charm decays to be identified topologically with a low background. This topological identification in addition to a momentum estimate of the parent charm momentum allowed inclusive production studies of single charm and charm pair distributions to be performed.

The goal of this thesis is present a complete analysis on the production properties of 37 cliarm pair events. The charm pairs were collected during the 1985 run of experiment E653 at the Fermi National Accelerator Laboratory (Fermilab).

Chapter Two of this thesis will briefly review the current theoretical status of charm production, followed by a review of the experimental measurements. The apparatus and its performance will be described in Chapter Three. The process of selecting charm, with minimal particle identification, will be discussed in Chapter Four. Chapter Five will present 
the background determinations. Chapter Six will describe the momentum estimator used in E653 production analysis and Chapter Seven will incorporate the momentum estimator into the Maximum Likelihood Fitting procedure used in this analysis. The final results are presented in Chapter Eight; followed by some concluding remarks in Chapter Nine. 


\section{Chapter 2}

\section{Hadro-Production of Charm: A}

\section{Review}

\subsection{Introduction}

Theoretical and experimental results on hadro-production of charmed particles have been converging on the same parton description of the fundamental interaction responsible for heavy quark production in hadronic interactions. This chapter will present a brief review of the theoretical aspects of heavy quark production with an emphasis on charmed particles. Following the theory will be a review of previous fixed target charm production results with an emphasis on charm pair results. More extensive reviews of heavy quark production, with an emphasis on charm production, can be found elsewhere $[39,40,41]$. 


\subsection{Theoretical Review}

\subsubsection{Total Charm Cross Section}

Heavy quarks produced in hadronic collisions are created by an interaction between the partons inside the interacting hadrons. The heavy quark cross section is modeled within the parton framework and is written as follows:

$$
\sigma\left(P_{1}, P_{2}\right)=\sum_{i j} \int d x_{i} d x_{j} \sigma_{i j}\left(\alpha_{8}(\mu), x_{1} P_{1}, x_{2} P_{2}\right) f_{i}\left(x_{1}, \mu\right) f_{j}\left(x_{2}, \mu\right)
$$

where $\sigma_{i j}$ represents the parton cross section and $f_{j}\left(x_{1}, \mu\right)$ represents the probability distribution of the $j^{\text {th }}$ parton at momentum fraction $x_{1}$. By using the factorization theorem unwanted singularities can be "factored" away [11.]. The short order cross section is calculated through a perturbative expansion of $\sigma_{i j}$ in powers of $\alpha_{\imath}(\mu)$. Nason, Dawson and Ellis [9] have used this method to calculate the charm cross section. The calculation was carried out to the next to leading order $\left(\alpha_{,}^{3}(\mu)\right)$. The leading order parton level diagrams are shown in Figure 2 and the next to leading order diagrams are shown in Figure 3. Berger has compared the next to leading order cross section to the leading order cross section by defining the $K$ factor as [13]:

$$
K=\frac{\sigma\left(\alpha_{a}^{2}(\mu)+\alpha_{s}^{3}(\mu)\right)}{\sigma\left(\alpha_{s}^{2}(\mu)\right)}
$$

The graph obtained from this calculation is shown in Figure 4. Previous leading order calculations ha' returned charm cross sections that were consistently lower than the measured values. This next to leading order calculation yields total cross section values that 

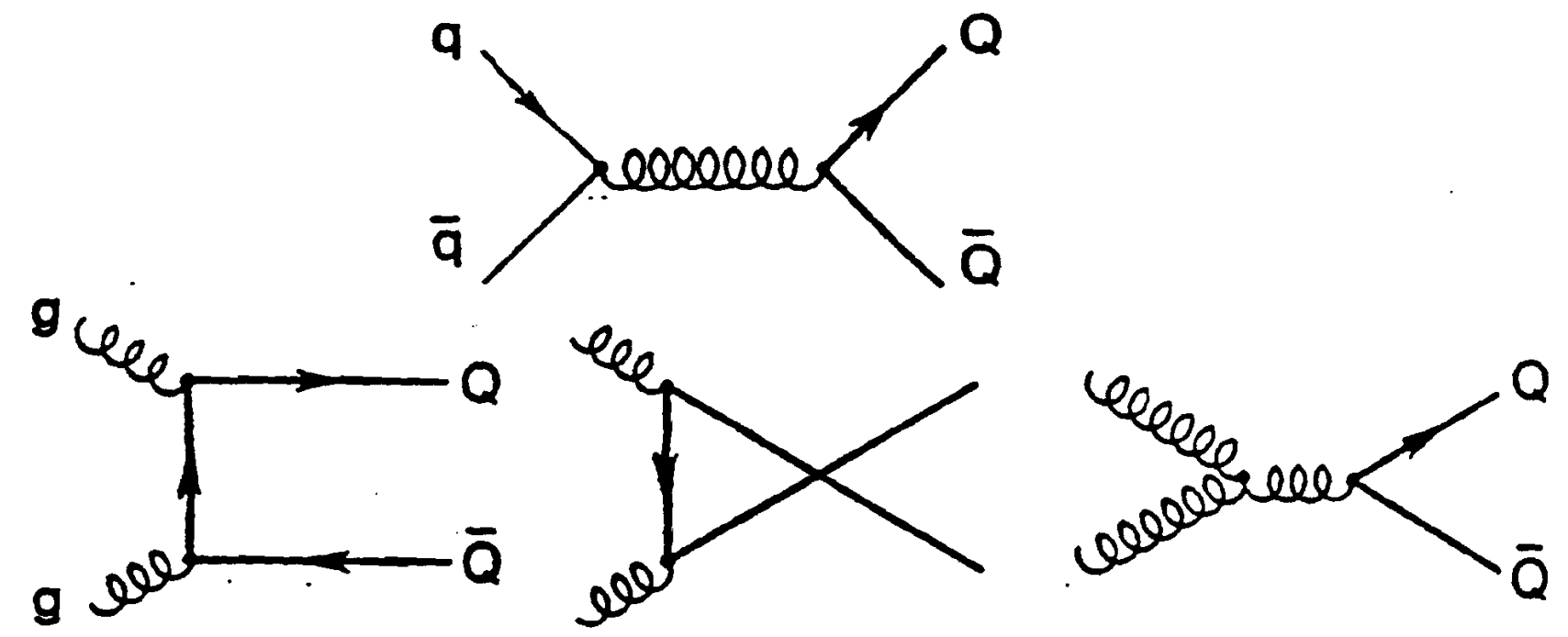

Figure 2: Leading order parton diagrams for heavy quark production.

are consistent with the measured cross sections without invoking a low charm quark mass or intrinsic charm component of the hadrons. Charm cross sections calculated through learling order diagrams required a very low charm mass or an intrinsic charm component in the incident hadrons.

The fact that the next to leading order diagram contribute heavily to the total cross section came as a surprise. However, Nason, Dawson and Ellis showed that some of the next. to leading order diagrams can contribute to the cross section at the same level as leading order diagrams. For example the cross section for $g g \rightarrow g g$ is approximately two orders of magnitude greater than the cross section for $g g \rightarrow q \tilde{q}$. This $g g \rightarrow g g$ process produces a heavy quark pair through gluon splitting (Figure 3c). 


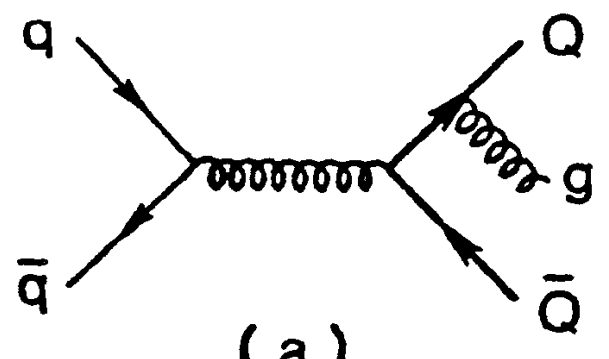

(a)
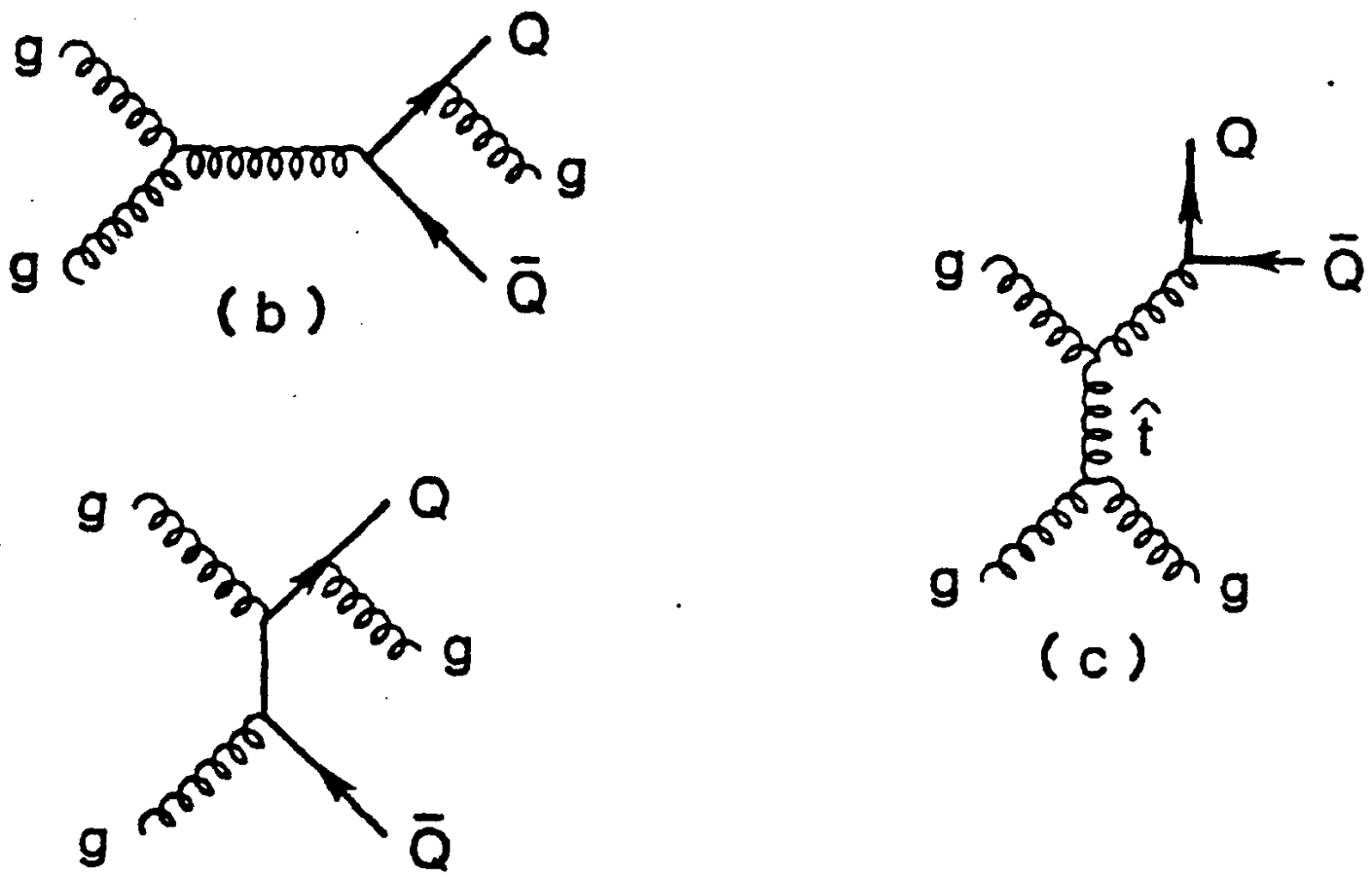

(d)

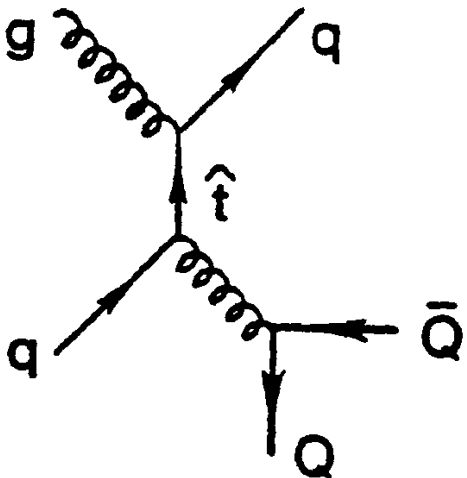

(e)

Figure 3: Ner to leading order pa: : nn diagrams for heavy quark production. 


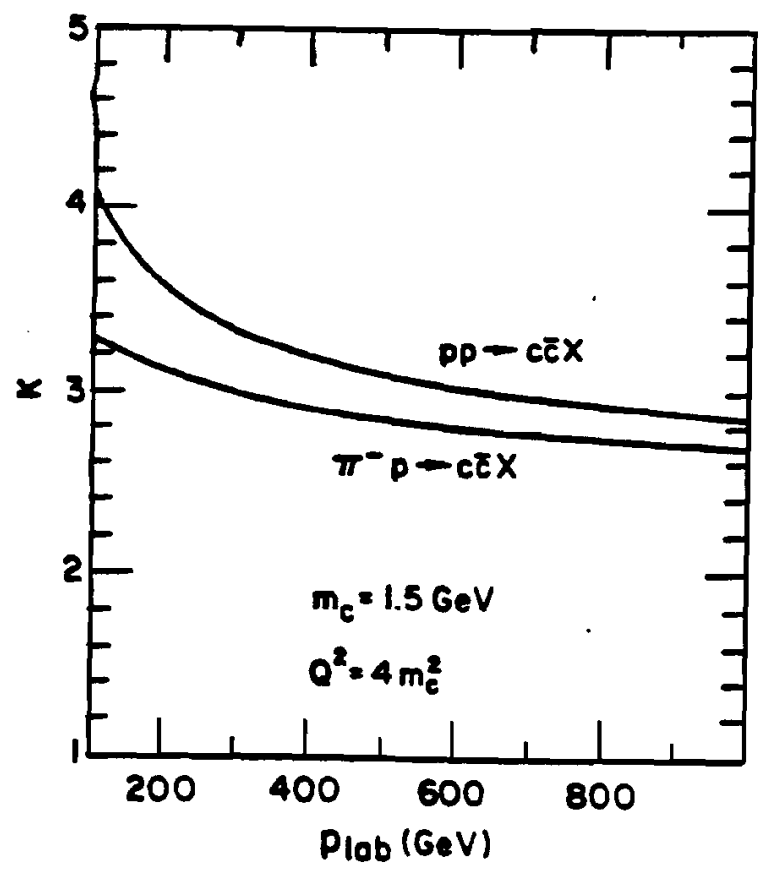

Figure 4: K-factor for the total charm cross section as a function of beam energy.

\subsubsection{Charm Correlations}

The cross section calculation provides a fundamental check of the theoretical basis of the parton model. In addition to the cross section there have also been predictions involving correlations between the $q$ and $\Phi$ produced in the interaction. Berger has shown that for quarks at least as massive of the beauty quark the rapidity gap ${ }^{1}(\Delta Y)$ of the quark pair has two distinct distributions depending on which diagram is contributing to the cross section [12]. For $q \bar{q} \rightarrow Q \bar{Q}$ the rapidity gap, $(|\Delta Y|)$, should be very small (full width at half maximum 1.3 for $|\Delta Y|)$. whereas for $g g \rightarrow Q \bar{Q}$ will be broad.(full width at half maximum of 3.0). Although these predictions are for beauty quarks they point out that the rapidity gap reflects the parton process responsible for heavy quark production. The

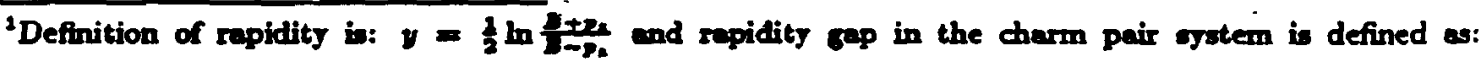
$\Delta Y=\left|Y_{0}-Y_{0}\right|$.
} 
differential heavy quark pair cross section was also shown to depend only on $\Delta \mathrm{Y}$ and not on the individual heavy quark rapidities, $y_{b}$ or $y_{6}$.

Another important charm pair variable is the square of the average charm pair momentum perpendicular to the beam direction, $\left\langle P_{t}^{2}\right\rangle[11]$ : Collins, Soper and Sterman predict that the measured value of $\left\langle P_{t}^{2}\right\rangle$ for charm pairs should be the same as that measured in Drell-Yan di-lepton production at the equivalent di-lepton mass. It. would also be interesting to see if the same type of $\left\langle P_{t}^{2}>\right.$ scaling that occurs in Drell-Yan production holds for charm pairs. The average $P_{t}^{2}$ scaling seen in Drell-Yan di-lepton production is:

$$
<P_{t}^{2}>=k_{t}^{2}+s \cdot \text { constant }
$$

The $k_{t}^{2}$ is a measure of the intrinsic transverse momentum of the partons involved in the process. Thus as Drell-Yan di-lepton production analysis was able to measure the intrinsic transverse momentum of the quarks involved in di-lepton production, charm pair $\left\langle P_{t}^{2}\right\rangle$ will.reflect the intrinsic transverse momentum of the gluons responsible for charm production.

\subsection{Hadronic Charm Production Results to Date}

Hadronic charm production experiments have been done with different beam and target particles, at different center of mass energies, and with different experimental tecluiques. This review will concentrate on fixed target hadro production charm experiments that have vertex resolution capabilities necessary to reconstruct chann decays. Table 1 lists the 


\begin{tabular}{lcccl}
\hline Experiment & $\begin{array}{c}\text { Beam } \\
\text { Momentum } \\
(\mathrm{GeV} / \mathrm{c})\end{array}$ & $\begin{array}{c}\text { Beam } \\
\text { Particle }\end{array}$ & $\begin{array}{c}\text { Target } \\
\text { Particle }\end{array}$ & $\begin{array}{l}\text { Vertex } \\
\text { Detector }\end{array}$ \\
\hline NA-32 & 200 & $\pi^{-}, \mathbf{K}, \mathbf{P}$ & $\mathrm{Si}$ & CCD's and silicon strips \\
E769 & 250 & $\pi^{-}, \mathbf{K}, \mathrm{P}$ & $\mathrm{Be}, \mathrm{Al}, \mathrm{Cu}, \mathrm{W}$ & Silicon strips \\
WA-82 & 340 & $\pi^{-}$ & $\mathrm{Si}, \mathrm{W}$ & Silicon Strips \\
NA-27 & 360 & $\pi^{-}$ & Proton & Bubble Chamber \\
NA-27 & 400 & Proton & Proton & Bubble Chamber \\
E743 & 800 & Proton & Proton & Bubble Chamber \\
E653 & 800 & Proton & Emulsion & Emulsion and silicon strips \\
\hline \hline
\end{tabular}

Table 1: List of fixed target charm experiments with hadron beams.

experiments in this review.

There are two experimental techniques used to obtain a sample of charm particles for production studies:

- Bubble chamber and muclear emulsion experiments where the event topology can be determined through visual methods $[36,49,50,53]$.

- High resolution silicon strip detectors that have sufficient resolution to reconstruct the decay vertices $[36,47,46]$.

The visual technique of the bubble chamber and emulsion yield small samples of charm decays with low backgrounds. The electronic experiments obtain large samples of charm decays with large combinatoric backgrounds that must be properly subtracted.

The production results from these experiments can be divided into four measurements, total charm cross section measurements, nuclear dependencs of the charm cross section, differential cross section distributions and charm pair distributions. The current status of these four measurements is presented in the following sections. 


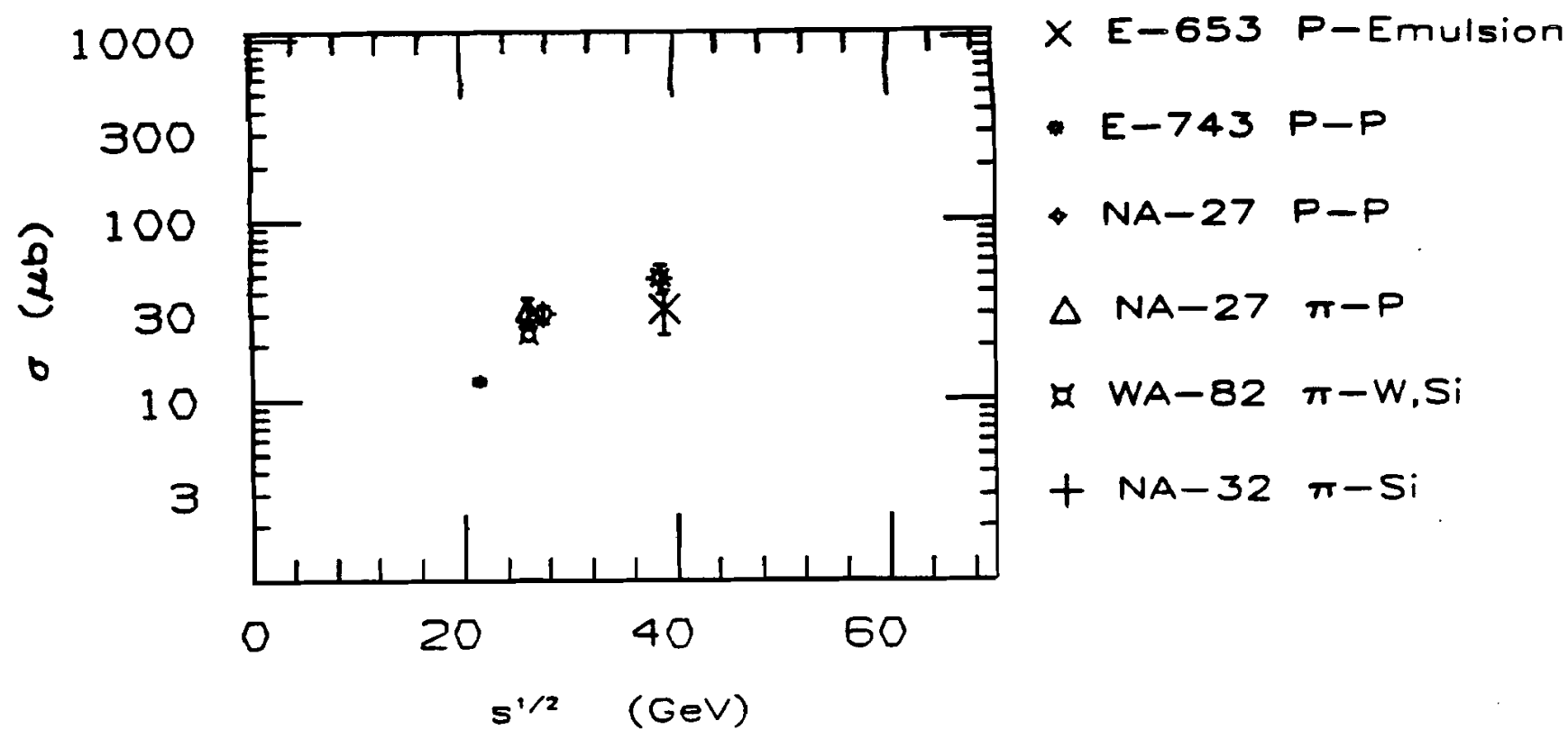

Figure 5: Total charm cross section measurements from different fixed target charm experiments as a function of center of mass energy.

\subsubsection{Total Charn Cross Section}

Until the theoretical calculation of the total cross section by Nason, Dawson and Ellis [9] the experimental cross sections did not aqree with those predicted by the leading order calculations. The measured cross sections are plotted in Figure 5 as a function of the center of mass energy $(\sqrt{s})[35,47,49,50,53,58]$. Cross sections for non-hydrogen targets were calculated assuming a linear A dependence of the cross section (see Section 2.3.2). The rise of the charm cross section with $\sqrt{s}$ agrees with the calculation of Nason, Dawson and Ellis, assuming a reasonable value for the charm quark mass $\left(m_{c}=1.5 \mathrm{GeV} / \mathrm{c}^{2}\right)$.

\subsubsection{A Dependence}

The factorization of the total cross section requires that the charm production process be described at the parton level and that the cross section be independent of the target nuclei. 


\begin{tabular}{lcccc} 
Experiments & Beam & Targets & $\alpha$ & Technique \\
\hline E613 & Proton & Be,Cu,W & $0.75 \pm 0.05$ & Beam Dump \\
WA78 & $\pi^{-}$ & Al,Fe,U & $0.80 \pm 0.05$ & Beam Dump \\
WA78 & Proton & Al,Fe,U & $0.78 \pm 0.09$ & Beam Dump \\
WA82 & $\pi^{-}$ & Si,W & $0.89 \pm 0.05 \pm 0.05$ & Reconstructed charm \\
E769 & $\pi^{-}$ & W,Cu,Si & $0.95 \pm 0.05$ & Reconstructed charm \\
\hline
\end{tabular}

Table 2: Measured values for $\alpha$ from different fixed target experiments.

This dependence on the target nuclei is usually parameterized as:

$$
\sigma=\sigma_{o} A^{\alpha}
$$

Early measurements of $\alpha$ done by studying the lepton spectrum from different beam dump targets, reported an $\alpha$ that was inconsistent with a parton description $[55,56,57]$. Recent results from fixed target experiments with fully reconstructed charm decays are consistent with $\alpha \sim 1.0[46,58]$. Table 2 list the measured values of $\alpha$. The A-dependence controversy will be settled when one experiment measures the parameter $\alpha$ for different regions of $\mathrm{X}_{f}$, since the beam dump experiments had acceptance only at large $\mathrm{X}_{f}$ and the reconstructed charm experiments are dominated by low $\mathrm{X}_{f}$ events.

\subsubsection{Differential Cross Section}

The charm differential cross section is parameterized by:

$$
\frac{d \sigma}{d X_{f} d P_{t}^{2}} \sim\left(1-\left|X_{f}\right|\right)^{n} e^{-b P_{t}^{2}}
$$


where:

$$
X_{f}=P_{\|} / P_{\| \max }[48]
$$

with:

$$
P_{\| \max }=\sqrt{\left(\frac{M_{i}^{2}-M_{r}^{2}+s}{2 \cdot \sqrt{s}}\right)^{2}-M_{i}^{2}}
$$

and:

- $\mathbf{P}_{\|}=$momentum of the charmed particle parallel to the beam direction

- $P_{t}=$ momentum of the charmed particle transverse to the beam direction

- $M_{i}=$ mass of the particle the for which $\mathrm{X}_{f}$ is being calculated

- $M_{r}=$ mass of the recoil particles in the reaction

Experiments quote results for $\mathrm{n}$ and $\mathrm{b}$ from their fits to the differential cross section. Figure 6 plots the measured value of $n$ versus the center mass energy for the fixed target experiments. The results from experiments with $\pi$ beams are all consistent with each other $[46,47,53]$. The $\mathrm{X}_{f}$ distribution from the LEBC-PP data is much flatter than the extremely central $\mathrm{X}_{f}$ distribution observed by E743 and E653 at higher $\sqrt{s}$. The measured value for the exponent $\mathrm{n}$ shows an increase in value at larger values of $\sqrt{s}$. As the energy of the incident proton beam increases a larger fraction of the gluon structure function is used in heavy quark production. The distribution observed by LEBC-PP is not as central as seen at higher $\sqrt{s}$ because only gluons with a large fraction of the proton momentum had enough energy to produce a charm pair. Comparing the $\pi$ beam data to the proton beam data shows that 


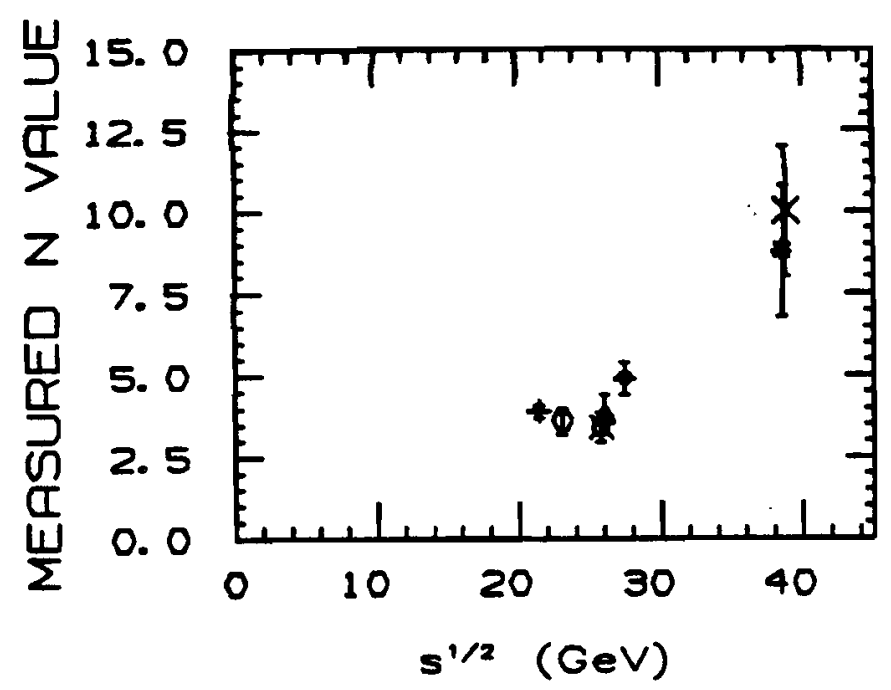

$\times$ E-653 P-Emulsion

- $E-743$ P-P

- NA-27 P-P

$\triangle N A-27 \pi-P$

A WA-82 $\pi-W, S i$

O $E-769 \pi-S i$

$+N A-32 \pi-S i$

Figure 6: The measured values for the exponent $n$ for the different fixed target experiments.

the $\mathrm{X}_{f}$ distribution is not as central for the $\pi$ data than for the proton data. This is due to the larger fractional momentun carried by the gluons distribution found in the $\pi$ compared to that found in the proton.

Theoretically the measured $\left\langle\mathrm{P}_{t}^{2}\right\rangle$ of the charmed particles should be approximately equal to the charm quark mass $\left(m_{c}^{2}\right)$. This prediction is borne out by the fixed target measurements. The $\left\langle\mathbf{P}_{t}^{2}\right\rangle$ from the different fixed target experiments is shown in Figure 7 . The measured values are all consistent with $\sim(1.0 \mathrm{GeV} / \mathrm{c})^{2}$, and do not show any trend with $\sqrt{s}$ or incident beam type.

\subsubsection{Charm Pair Results}

Single charm production studies have yielded important results on the A-dependence and the total charm production cross section, but it is difficult to determine the details of 


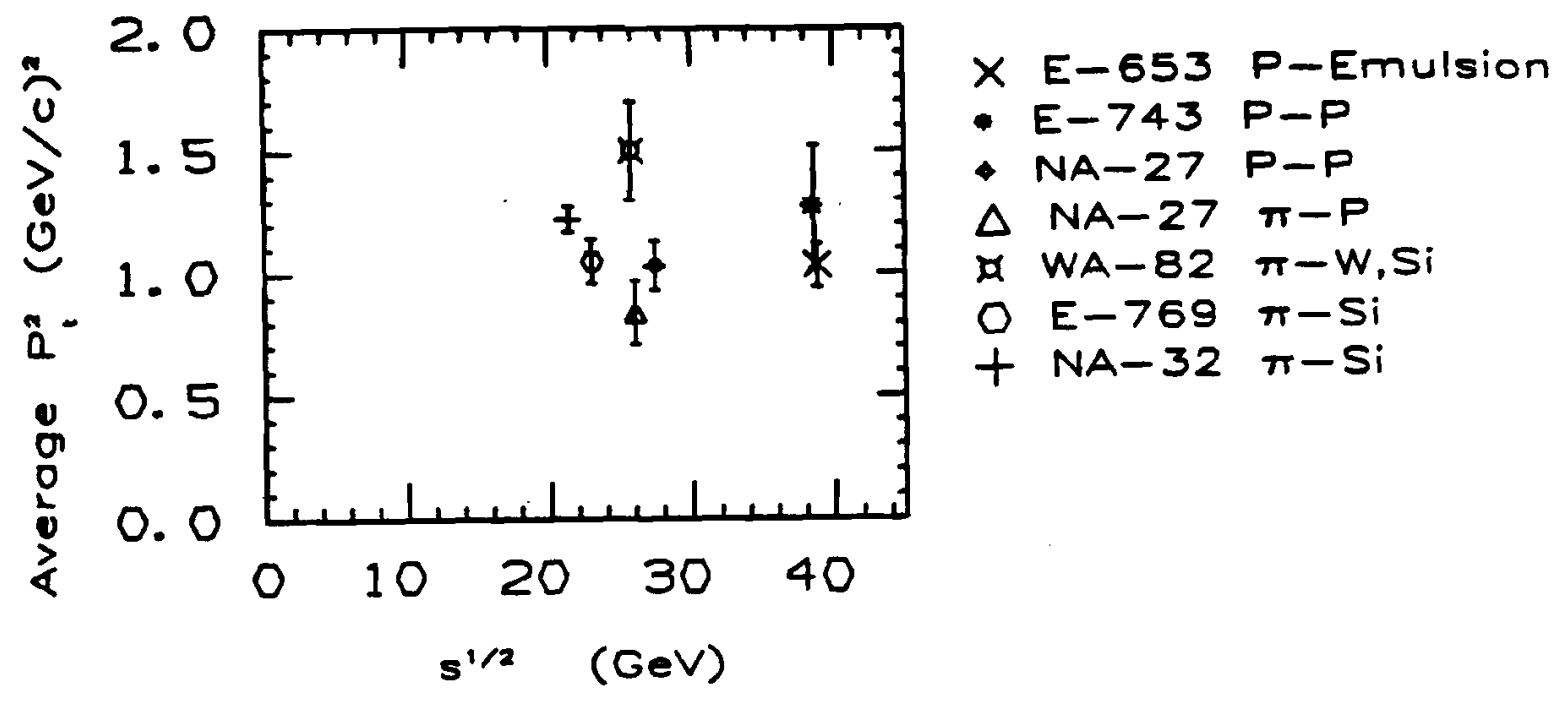

Figure 7: The mearured values for the $\left\langle P_{t}^{2}\right\rangle$ from the different fixed target experiments.

charm production from single charm production distributions. It is hoped that by studying the kinematics and dynamics within the charm pair system the parton (gluon) structure functions, fragmentation effects and contributions from specific production diagrams can be determined. The analysis would follow the Drell-Yan analysis of di-lepton production in hadron interactions. The Drell-Yan di-lepton analysis and results will be discussed in the next. section, but let me remind the reader that the quark and anti-quark structure functions for protons and $\pi$ 's, and the intrinsic transverse momentum $\left(k_{t}\right)$ of the quarks was determined through Drell-Yan analysis. A sample of charm pairs with sufficient statistics and momentum resolution is required for this analysis and the two published samples of charm pairs do not have enough events for such elaborate analysis.

The LEBC experiment collected two sets of charm pairs $[51,52]$. One set of 12 charm pairs was collected using a $360 \mathrm{GeV} / \mathrm{c} \pi$ - beam incident on the bubble chamber filled with 
liquid hydrogen. (These results will be referred to as LEBC- $\pi$ P throughout this thesis.) A second run with a $400 \mathrm{GeV} / \mathrm{c}$ proton beam collected 17 charm pair events. (Referred to hereafter as LEBC-PP.)

The LEBC- $\pi \mathrm{P}$ and LEBC-PP analyses compared the weighted charm pair $\mathrm{X}_{f}, \mathrm{P}_{t}^{2}$, mass, azimuthal opening angle $\left(\Phi_{t}\right)$ and rapidity gap $(\Delta Y)$ distributions with the distributions from charm pairs generated by the LUND Monte Carlo (LUND MC). The LUND MC is a Monte Carlo of high $P_{t}$ physics in various interactions. The program allows the user to define the incident particles and energies, structure functions of hadrons and the fragmentation functions used to add light quarks to the bare charm quarks to make charmed mesons and baryons. The LUND MC, however, only produces charm pairs via the leading order diagrams.

The LEBC- $\pi$ P and LEBC-PP pairs distributions agreed quite well with the pairs generated via the LUND MC. The LEBC results agreed best when the intrinsic transverse parton momentum was parameterized by;

$$
\frac{d N}{d k_{t}^{2}}=e^{\left.-k_{i}^{2} /<k_{1}^{2}\right\rangle}
$$

with:

$$
<k_{t}^{2}>=0.64(\mathrm{GeV} / \mathrm{c})^{2}
$$

This value for $<k_{t}^{2}>$ was taken from Drell-Yan studies [21]. The parton structure functions of Duke and Owens and the LUND fragmentation scheme were seen by the LEBC collaboration to be superior to other sets of structure functions and fragmentation schemes 


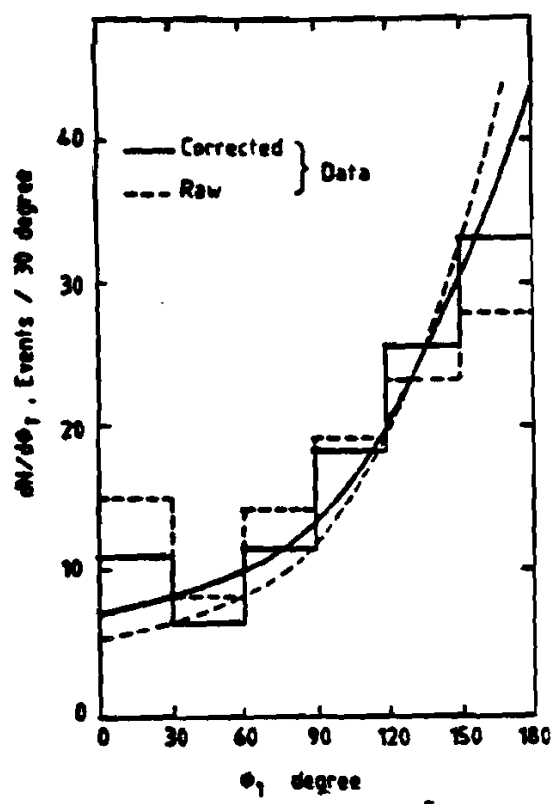

Figure 8: The $\Phi_{t}$ distribution measured by LEBC-PP. There are 107 geometrically reconstructed pairs in the plot.

$[15,51]$.

The IEBC-PP results are shown in Figures 8 and 9. The agreement between the data and the LUND MC charm pairs is quite good. The aximuthal opening angle, $\Phi_{t}$, of the charn pairs shows the expected peaking at $180^{\circ}$. This peaking reflects the back-to-back nature of the leading order production diagrams. For the LUND MC $\Phi_{t}$ distribution to agree with their data the $\left\langle k_{t}^{2}\right\rangle$ of the partons was set to the maximum value consistent with Drell-Yan di-lepton results. The charm pair mass, $X_{f}$ and $\Delta Y$ distributions agree quite well with the distributions generated by the LUND MC. The $P_{t}^{2}$ distribution shows a elight tail that is not reproduced by the LUND MC.

The agreement between LUND MC and the LEBC-PP data is hard to resolve in light of the Nason, Dawson and Ellis cross section calculation. The theoretical charm cross section did not reproduce the measured cross sections until the next-to-leading order production 

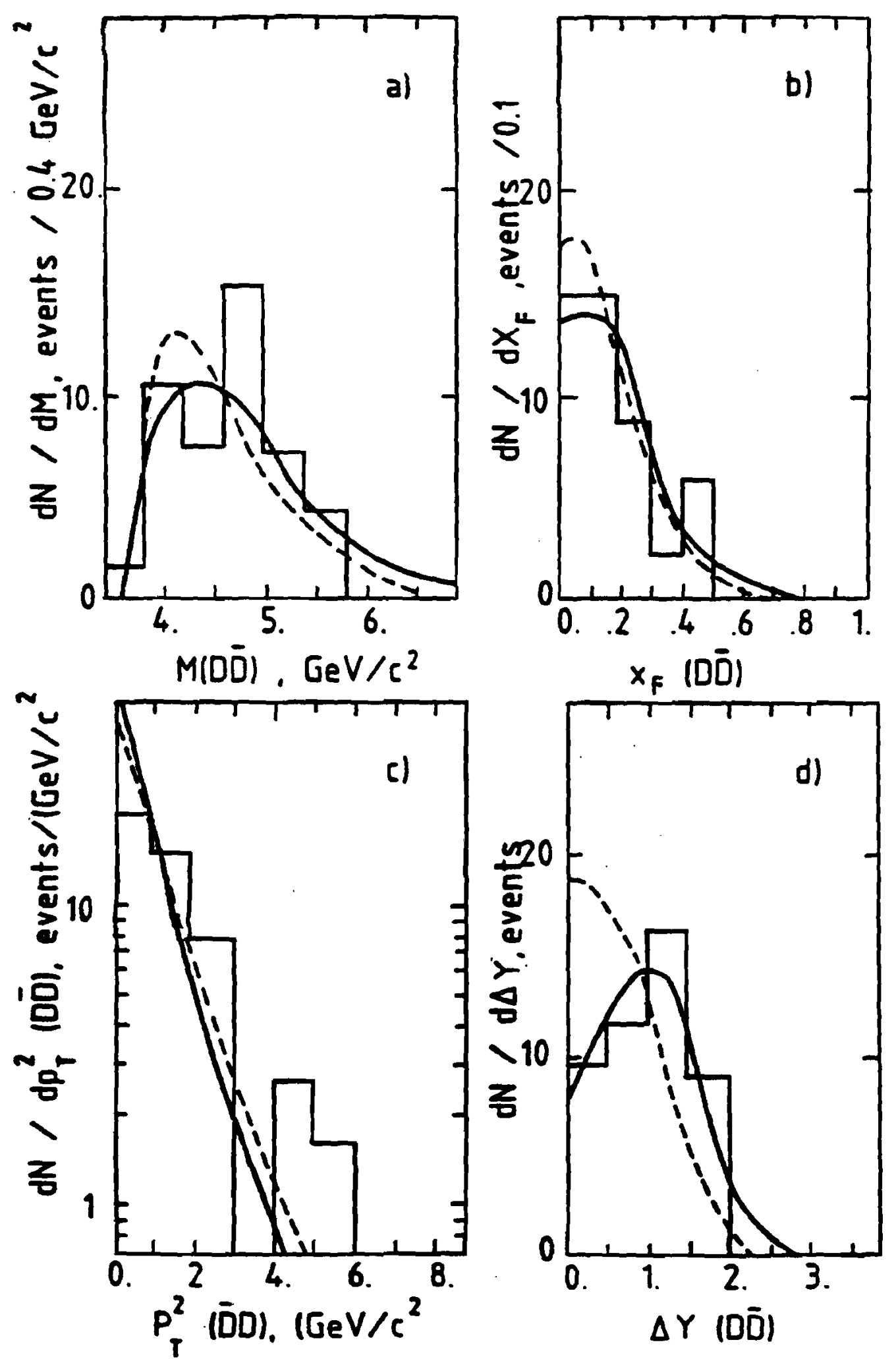

Figure 9: The weighted $X_{f}$, mass, $P_{t}^{2}$ and $\Delta Y$ distributions for the 17 LEBC-PP charm pairs. The dashed curve is from the LUND MC with a $\delta$ fragmentation function and the solid curve is the LUND MC with I,IIND fragmentation. 
diagrams were included in the calculation. It could be that the next-to-leading order diagrams contribute only at large $Q^{2}$ or mass and the LEBC- $\pi$ P or LEBC-PP data has not probed that region. A high statistics charm pair experiment with large mass charm pair events is needed to deternine the exact role of the production diagrams for leading and next to leading orders.

\subsection{Drell-Yan Di-Lepton Production}

\subsubsection{Introduction}

The purpose of this section is not to give an extensive review of Drell-Yan results, but. to briefly state some of the scaling observed in the Drell-Yan experiments. Complete and detailed reviews can be found elsewhere $[17,20]$.

\subsubsection{The Drell-Yan - Charm connection}

The mechanism first proposed by S. Drell and T. Yan for the continuum di-lepton production in hadronic interactions is a quark-antiquark annihilation into a virtual photon that splits into a lepton and anti-lepton pair (Figure 10) [19]. This simple parton description has been very successful in describing the experimental results. The factorization theorem is complete for the Drell-Yan process [11] and the theoretical understanding of di-lepton production is on firm ground. 


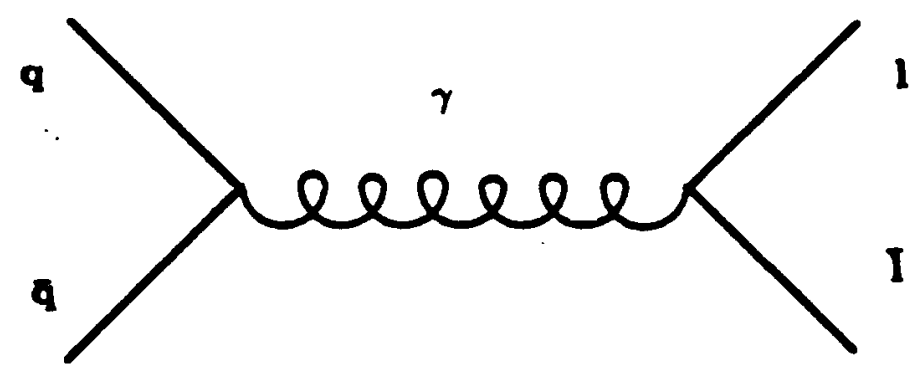

Figure 10: Leading order production diagram for di-lepton production in hadronic interactions. 


\section{Total Cross section}

The initial total cross section calculations for di-lepton production were lower than the experimental cross sections by more than a factor of two. This discrepancy was accounted for when the next to leading order production diagrams were included in the cross section calculation. This is very analogous to the recent results on the theoretical calculation of the charm cross section. The equation for the prodution cross section is similar to that written for heavy quark production with the number of partons summed over reduced to the quark and anti-quark partons. The perturbative expansion is done through powers of $\ln Q^{2} / \mu^{2}$ where $Q^{2}$ represents the di-lepton mass squared.

\section{A-dependence}

Since di-lepton production in a hadronic environment is perceived as a parton-parton interaction, the total cross section should be independent of the target composition. The $\alpha$ value measured with $\pi$ and proton beams is consistent with $\alpha=1.0$. The recent measurements from charm production experiments are also consistent with $\alpha=1.0$ and the premise of $a$ short order parton-parton interaction being responsible for both di-lepton production and heavy quark production appears to be valid.

\subsubsection{Drell-Yan Scaling}

The parton description of the di-lepton process simplified the theoretical calculation and allowed for detail predictions of how measu-ed quantities shoula scale. The prominent scaling observed in di-lepton experiments will be described here along with some of the 
important results.

\section{Di-Lepton Mass Scaling}

By introducing the dimensionless parameter:

$$
\tau=\frac{m_{l}^{2}}{s}=x_{i} \bar{x}_{j}
$$

the differential cross section was seen to scale as:

$$
s \frac{d^{2} \sigma}{d y d \sqrt{\tau}}=\text { const } \cdot e^{-a \sqrt{\tau}}
$$

This scaling allowed experiments performed at different center of mass energies to compare results by comparing their respective values of $a$. The experimental results were seen to scale over almost two orders of magnitude of $\sqrt{\tau}$. If this scaling holds for charm pairs it will provide a way for different charm pair experiments at different energies to compare results. The value for the exponent for $400 \mathrm{GeV} / \mathrm{c}$ proton-platinum interactions is $a=27.04 \pm 0.17$ [22]. This scaling also reflects the probability distribution of the product of the momentum fractions, $x_{i} \bar{x}_{j}$.

\section{A verage $\mathbf{P}_{t}^{2}$}

The average $\mathbf{P}_{t}^{2}$ of the di-lepton pair was predicted to scale as:

$$
<P_{t}^{2}>=<k_{t}^{2}>+\alpha_{\bullet}\left(Q^{2}\right) f\left(\tau, X_{f}, \ln Q^{2}\right) \cdot s
$$


where $k_{t}^{2}$ represents the intrinsic transverse momentum of the partons and $\mathrm{X}_{f}$ is the dilepton $\mathrm{X}_{f}$. Thus at constant $\tau, \mathrm{X}_{f}$ and $\mathrm{Q}^{2}$ the relationship is linear:

$$
<P_{t}^{2}>_{\tau, X_{f}, Q^{2}}=<k_{t}^{2}>+C_{s l} \cdot s
$$

This analysis has been done [21] and has yielded:

$$
\begin{gathered}
<k_{t}^{2}>=0.52 \pm 0.12(\mathrm{GeV} / \mathrm{c})^{2} \\
C_{s l}=0.0014 \pm 0.0002\left(\mathrm{GeV} / \mathrm{c}^{2}\right)
\end{gathered}
$$

for protons and

$$
\begin{gathered}
\left\langle k_{t}^{2}>=0.59 \pm 0.05(\mathrm{GeV} / \mathrm{c})^{2}\right. \\
C_{s l}=0.0028 \pm 0.0002\left(\mathrm{GeV} / \mathrm{c}^{2}\right)
\end{gathered}
$$

for $\pi$ 's.

Since Drell-Yan production proceeds through quark-antiquark annihilation the $\left\langle k_{t}^{2}\right\rangle$ measured is that of the quarks and antiquarks. Charm pair analysis would measure the intrinsic $k_{t}^{2}$ of the gluons that dominate the charm pair production process.

\subsubsection{Structure Functions}

Large statistic di-lepton experiments were able to determine the structure functions of the quark and anti-quarks inside the incident proton and pions. This was done by studying 
di-lepton mass distributions at constant pair rapidity. For $\pi p$ interactions the cross section is dominated at large $x_{p}$ and $x_{\pi}$ by the product of the structure functions, $f_{a}^{\pi}\left(x_{\pi}\right)$. The structure functions of the sea quarks determined in deep inelastic lepton scattering for the proton, $f_{u}^{p}\left(x_{p}\right)$, are used to unfold the structure function of the incident pion, $f_{\tilde{u}}^{p}\left(x_{\pi}\right)$. In the case where the structure functions of both particles are unknown the structure functions can be extracted by studying the di-lepton mass distribution at $\mathrm{X}_{f}(l \bar{l})=0$.

The same analysis can be applied to high statistics charm pair data and the structure functions of the gluons in protons, pions and kaons can be measured. This might be the only way to directly measure these distributions.

The di-lepton production analysis has made important measurements of the quark distributions inside the incident hadrons. It has also provided a test of perturbative QCD. Charm pair production holds the same promising future however both the experimental and theoretical aspects of charm pair production are much more complicated that those for di-lepton production. 


\section{Chapter 3}

\section{The Hardware}

\subsection{Introduction}

E653 was designed to study the properties of charm production in hadronic interactions and their subsequent decays. The known characteristics of hadronic interactions and charm decays were used to optimize the expected charm yield.

These considerations were:

- The ratio of the charm cross section to the total cross section in hadronic:photon:neutrino: $\mathrm{e}^{+} \mathrm{e}^{-}$interactions is approximately $0.001: 0.01: 0.1: 1$.

- Typical charm meson lifetimes were then measured at:

$$
\begin{aligned}
& \operatorname{cr}\left(D^{ \pm}\right)=0.320 \pm 0.010 \mathrm{~mm} \\
& \operatorname{cr}\left(D^{\circ}\right)=0.1283 \pm 0.0033 \mathrm{~mm}
\end{aligned}
$$


- The semi-leptonic branching ratios for heavy quarks were measured to be large and roughly $10 \%$ [25].

- Average charged track multiplicity in hadronic interactions is large, approximately 15 tracks per event.

The solution to these hard technical problems was the following:

- An emulsion target with $1 \mu \mathrm{m}$ spatial resolution could resolve the short decay lengths characteristic of charmed particles and handle the high charged track density.

- High resolution silicon strip detectors would aid in selecting charm events and predicting their location in the emulsion.

- A Muon trigger to select events with a high momentum muon, thereby reducing the non-charm background.

- Reconstruct this trigger muon throughout the spectrometer and use the resolution of the silicon strip detectors to determine if the muon is consistent with coming from a decay.

These were the primary ideas behind the E653 hybrid emulsion-spectrometer technique. The plan view of the complete experiment is shown in Figure 11. Each detector had its own internal geometry and coordinate system, which had to be converted to the chosen E653 coordinate system shown in Figure 12. The E653 coordinate system was a right handed system with the $\mathrm{Z}$-axis along the beam direction, see Figure 12 . The hardware that was used consisted of the following: 


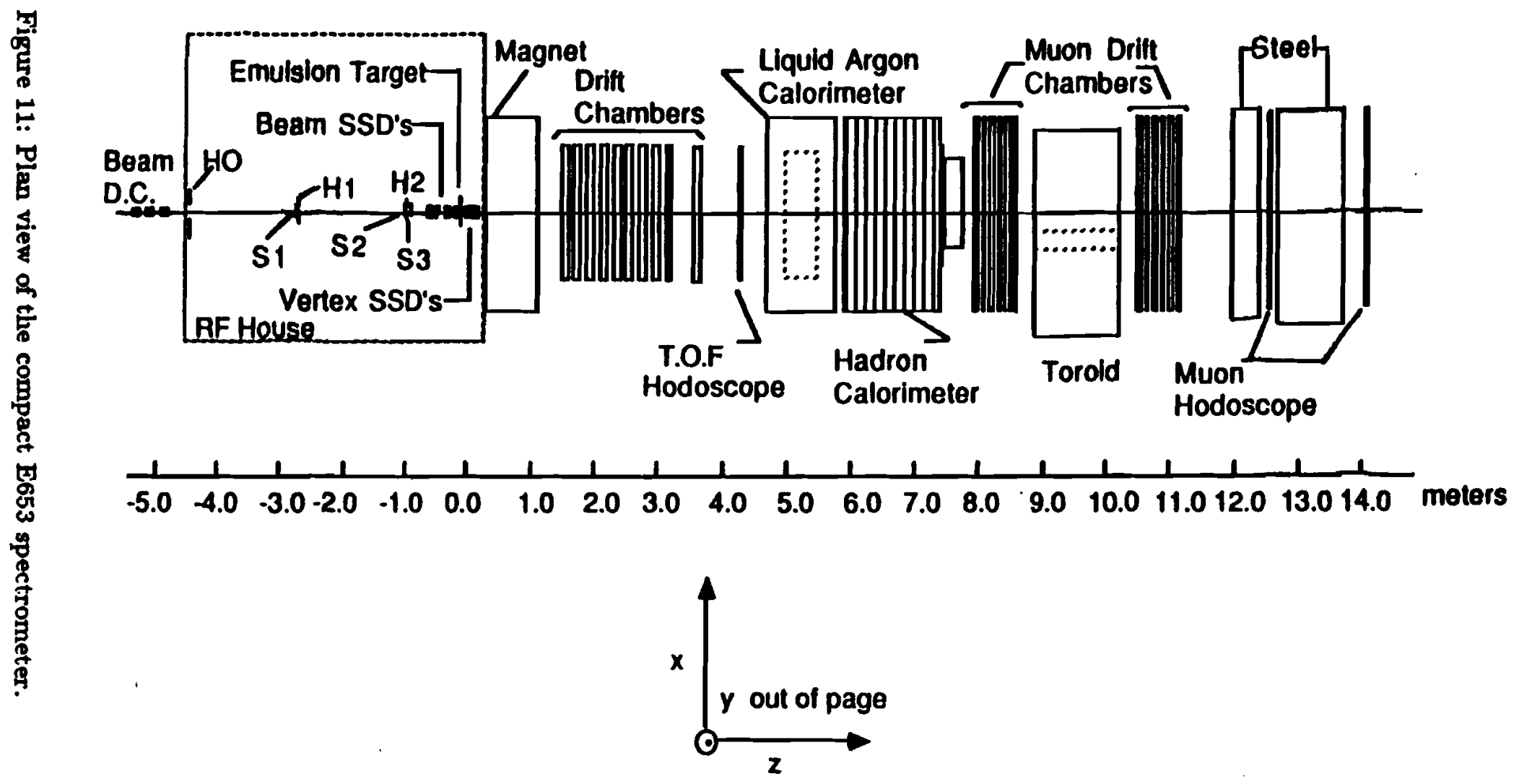




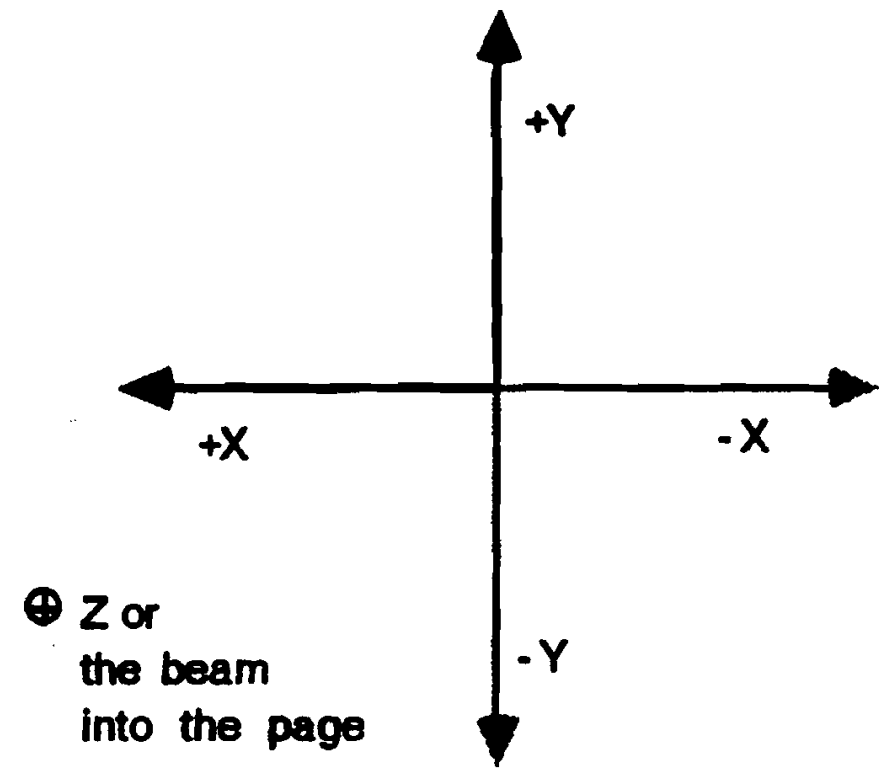

Figure 12: The E653 coordinate system.

- Beam system: The beam proton trajectory was determined before it enter the emulsion by a drift chamber system coupled with 9 planes of $20 \mu \mathrm{m}$ pitch silicon strips. The beam track provided an important starting point in reconstructing the primary vertex.

- Emulsion target: There were two types of emulsion targets, vertical and horizontal, with $14 \mathrm{~mm}$ and $20 \mathrm{~mm}$ thickness, respectively. The emulsion targets were uniformly exposed by moving the emulsion during data taking.

- Vertex silicon strip detectors: Following the emulsion were 18 planes of $50 \mu \mathrm{m}$ pitch silicon strip detectors. The vertex silicon strip detectors (VSSD's) measured the charged track trajectories upon leaving the emulsion target.

- Analyzing magnet and vector drift chambers: The charged tracks were then bent by 
the magnetic field of the analyzing magnet and the new trajectories were measured by 10 vector drift chambers (SDC's). Track linking between the VSSD's and SDC's provided the momentum measurements for the charged tracks.

- Time of flight, liquid argon calorimeter, and hadron calorimeter: Low momentum particles were identified by the time of flight (TOF) system immediately downstream of the SDC's. Followiug the TOF system was the liquid argon calorimeter (LAC) that provided electron identification, $\gamma$ tagging and $\pi^{\circ}$ reconstruction. Long lived hadrons deposited their energy into the hadron calorimeter that followed the LAC.

- Muon spectrometer and trigger: Following the hadron calorimeter was the muon spectrometer which consisted of ranging steel, 12 drift chambers, a toroidal magnet, and the muon hodoscope. The muon spectrometer provided a completely independent measurement of the muon momentum crucial in linking this track to the upstream spectrometer. The muon trigger was accomplished by requiring a coincidence between the front and back sections of the muon hodoscope- that followed the muon spectrometer.

Of course there are always sacrifices to be made when designing an experiment and this case the notable compromises in the design were (hindsight is a wonderful thing):

- The multiple scattering in the thick emulsion target would reduce the vertex resolution of the VSSD's.

- For the muon trigger to have a large acceptance and to reduce the number of $\mu$ 's from $\pi$ clecay the spectrometer must be short (Total length from target to muon hodoscope 


\begin{tabular}{|l|c|}
\hline Subsystem & Reference \\
\hline Overall & {$[28,30,36]$} \\
\hline Emulsion & {$[34]$} \\
\hline VSSD's & {$[28]$} \\
\hline SDC's & {$[27]$} \\
\hline LAC & {$[26,31]$} \\
\hline Hadron calorimeter & {$[32]$} \\
\hline Muon Spectrometer & {$[29,33]$} \\
\hline Data Acquisition & {$[30]$} \\
\hline
\end{tabular}

Table 3: References for the E653 spectrometer and its components.

was $15 \mathrm{~m}$ ). This short spectrometer does not allow the use of Cerenkov detectors for particle identification.

- The muon trigger forces one of the decay particles to be semi-leptonic. This decay due to associated neutrino daughter cannot have a precise momentum determination.

The muon trigger has turned out to be an exceptional advantage in studying of the semi-leptonic decays of charn mesons.

It is the purpose of this chapter to describe the apparatus needed for the charm pair production analysis (Beam system, emulsion target, charged particle spectrometer, and the muon spectrometer). The E653 apparatus has been described in detailed elsewhere, and I would at this time direct the reader to the theses and papers in Table 3 for complete detailed information on the E653 einulsion-spectrometer. 


\subsection{Beam Line}

E653 ran with the highest possible proton beam energy available to take advantage of the dependence of the charm cross section on the center of mass energy (see Figure 5). In 1985 this meant using the $800 \mathrm{GeV}$ proton beam available from the Fermilab accelerator. The intensity of the proton beam was controlled by a triangular cross section shaped tungsten attenuator and ranged between $10^{4}$ and $10^{7}$ protons/sec. The beam spot was required to be less than $3 \mathrm{~mm} \times 3 \mathrm{~mm}$ in area, to ensure that the region of highest track density passed through the central region of the electronic spectrometer. The beam halo, integrated over $10 \mathrm{~cm}^{2}$, was required to be less than $7 \%$ of the beam intensity. The small halo was necessary to ensure that the emulsion did not become blackened by uninteresting tracks. The beam line chosen for E653 was the NE beam line shown in Fig. 13 of the Fermilab accelerator and beam line complex.

\subsection{Beam System}

The beam proton's trajectory was determined by the E653 beam system. The beam system consisted of two independent detectors, the beam drift chambers (BDC's) $500 \mathrm{~cm}$ upstream of the emulsion and the beam silicon strip detectors (BSSD's) situation $27 \mathrm{~cm}$ upstream of the emulsion target. The beam track was an important part of the primary vertex reconstruction since it limited the window transverse to the beam direction in which the primary interaction occurred.

The upstream (BDC) element of the beam system was a conventional drift chamber 
要

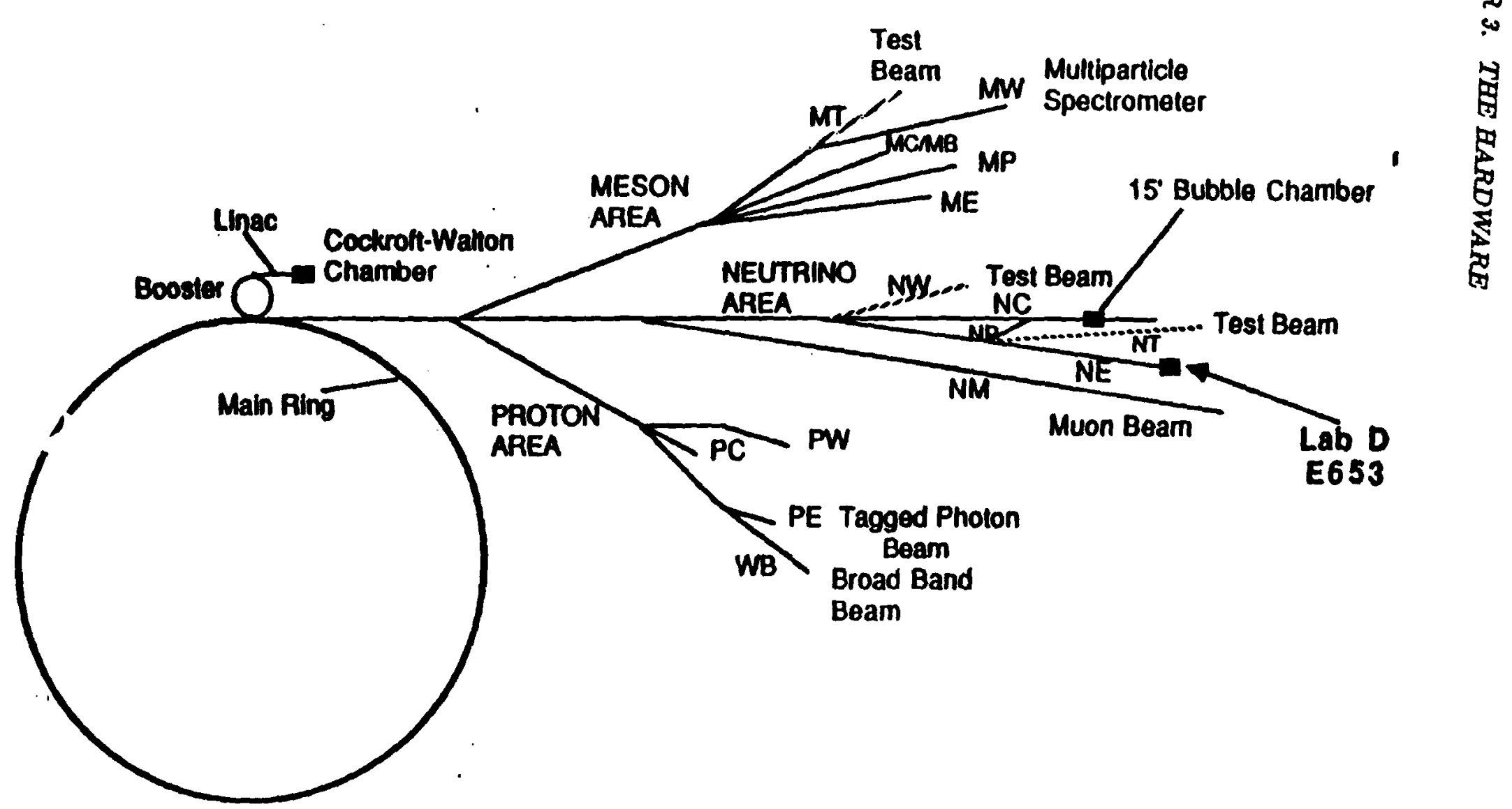


composed of 18 planes in three different views, $X, U$ and $V$, with the $U$ and $V$ views making a $\pm 60^{\circ}$ angle with the $X$ axis. Each plane had two sense wires per plane. The planes were grouped in pairs with the same orientation but with the wires offset within the pair to aid in unfolding ambiguous tracks. The resolution of the BDC was $150 \mu \mathrm{m}$ (microns)/plane.

The BSSD's located $27 \mathrm{~cm}$ in front of the target provided another measurement of the beam position. There were 9 planes of silicon strip detectors with 20 micron pitch in the BSSD system. The planes were arranged in the same XUV projections as the BDC's. Only the center $1.24 \mathrm{~cm}$ region of the $3.4 \mathrm{~cm} \times 3.6 \mathrm{~cm}$ detectors was implemented since the beam was confined to a $3 \mathrm{~mm} \times 3 \mathrm{~mm}$ spot size. This central region of the silicon strips had a position resolution of 10 microns.

The complete beam system had an overall angular resolution of $20 \mu \mathrm{rad}$ and a position resolution of 10 microns. Figure 14 shows the beam spot and slope as measured by the beam system.

\subsection{Emulsion Target}

The emulsion target provided the target nuclei for the incident proton and served as a detector for the outgoing charged tracks. These two uses for the emulsion defined its design criteria. The emulsion target had to be thick enough to provide a reasonable rate of primary interactions yet thin enough so that the products of the primary interaction could escape the emulsion without considerable multiple scattering. A thin emulsion would also reduce the probability of a track from the primary interaction interacting with an emulsion nucleus 


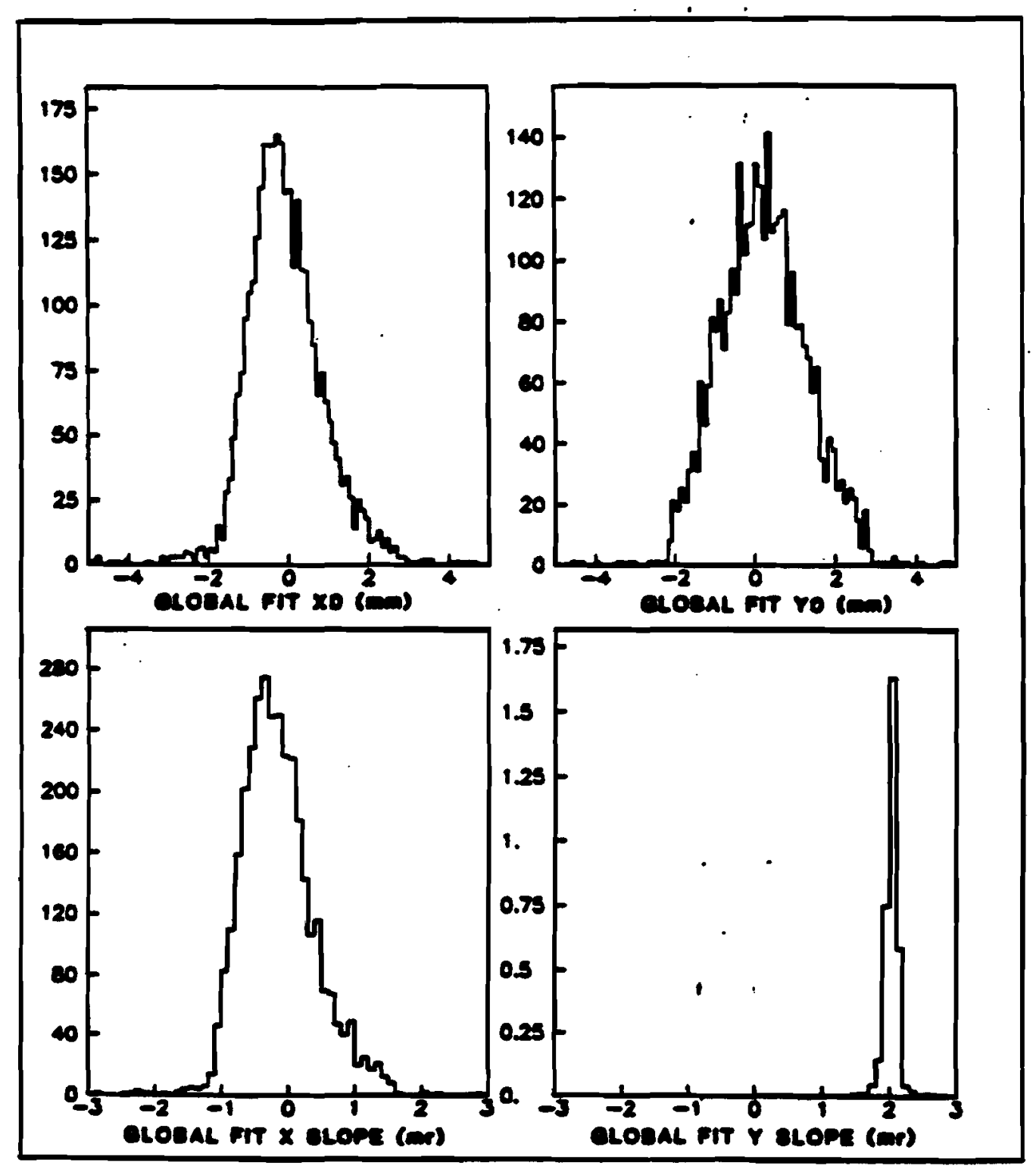

Figure 14: The integrated beam spot of the $800 \mathrm{GeV}$ proton beam measured by the beam system. 


\begin{tabular}{|l|r|r|r|r|}
\hline \hline Element & Weight \% & Atomic Weight & Atomic Number & Number \% \\
\hline$(\mathrm{i})$ & $\left(\mathrm{W}_{i}\right)$ & $\left(\mathrm{A}_{i}\right)$ & $\left(\mathrm{Z}_{i}\right)$ & $\left(\mathrm{N}_{i}\right)$ \\
\hline $\mathrm{I}$ & 0.3 & 126.90 & 53 & 0.1 \\
\hline $\mathrm{Ag}$ & 45.4 & 107.87 & 47 & 11.7 \\
\hline $\mathrm{Br}$ & 33.4 & 79.90 & 35 & 11.1 \\
\hline $\mathrm{S}$ & 0.2 & 32.06 & 16 & 0.2 \\
\hline $\mathrm{O}$ & 6.8 & 16.00 & 8 & 11.3 \\
\hline $\mathrm{N}$ & 3.1 & 14.01 & 7 & 5.9 \\
\hline $\mathrm{C}$ & 9.3 & 12.01 & 6 & 20.6 \\
\hline $\mathrm{H}$ & 1.5 & 1.01 & 1 & 39.6 \\
\hline \hline Totals & 100.0 & $\langle\mathrm{~A}\rangle=26.64$ & $\langle\mathrm{Z}\rangle=12.42$ & 100.0 \\
\hline \hline
\end{tabular}

Talble 4: Chemical composition of Fuji ET-7B nuclear emulsion. The value for $\langle A\rangle$ was calculated assuming a linear dependence on $A$.

(secondary interactions), and the number of ganuna conversions.

Nuclear emulsion is always active. This meant that the amount of stray (out of BSSD, VSSD acceptance) protons or halo in the beam had to be kept to a minimum (less than $7 \%$ of the total intensity). The emulsion was moved throughout the beam spill at a constant rate to insure uniform exposure of the enulsion block.

The nuclear emulsion used in E653 was Fuji E7- 7 B. The chemical composition is given in Table 4 taken from [30] along with the calculation for the average effective atomic weight. This calculation was done assuming a linear $\mathrm{A}$ dependence.

During the Run I (1985) 32 liters of enulsion were exposed in four months of running. This corresponded to 49 target modules. The target modules came in two different configurations, vertical and horizontal. 


\subsubsection{Vertical Emulsion Module}

Most (71\%) of the data was taken with vertical emulsion targets. The vertical targets consisted of a closely spaced region, the main emulsion block, of emulsion sheets (called pellicles) and followed by a region of spaced out pellicles, the analyzing region. The pellicles were oriented perpendicular to the beam. Figure 15 shows the configuration of the pellicles for a vertical module. The vertical modules represented 0.043 interaction lengths and 0.473 radiation lengths of material. The analyzing region allowed the emulsion scanners to follow tracks far from the primary interaction and locate daughter tracks from decays from long lived particles. The density of beam proton tracks for the vertical modules was $1.5 \times 10^{5}$ protons $/ \mathrm{cm}^{2}$ this corresponds to an average incoming beam track separation of $26 \mu \mathrm{m}$. This separation was enough for the enulsion scanners to distinguish separate events.

\subsubsection{Horizontal Emulsion Module}

Figure 16 shows the configuration of the horizontal emulsion modules. The beam was incident on the edge of the pellicles. The main horizontal emulsion block was also followed by a analyzing region of spaced out emulsion sheets. A horizontal module represented 0.064 interaction lengths and 0.719 radiation lengths of material. The density of beam proton tracks was $0.5 \times 10^{5}$ protons $/ \mathrm{cm}^{2}$ for the horizontal module. This track density corresponds to an average incoming beam track separation between $77 \mu \mathrm{m}$. 


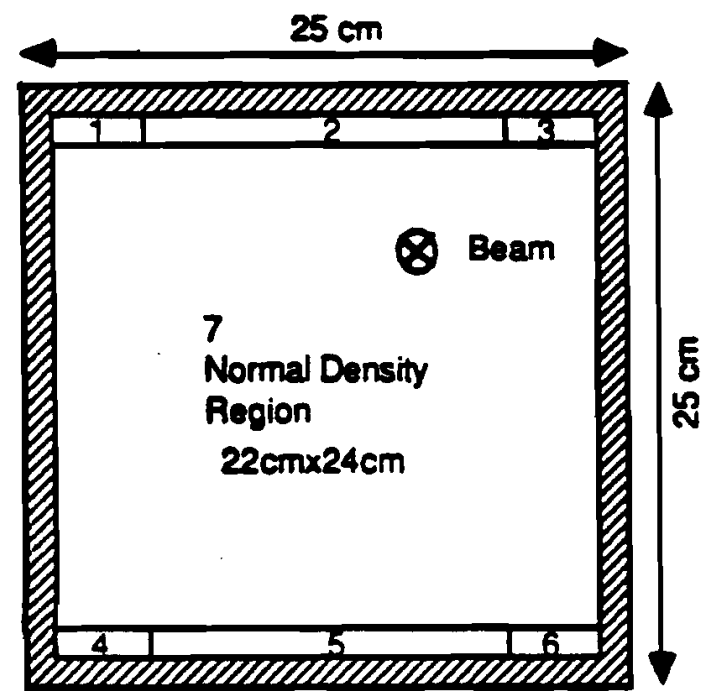

2, 5: Low Density Regions

$1,3,4,6:$ Spot

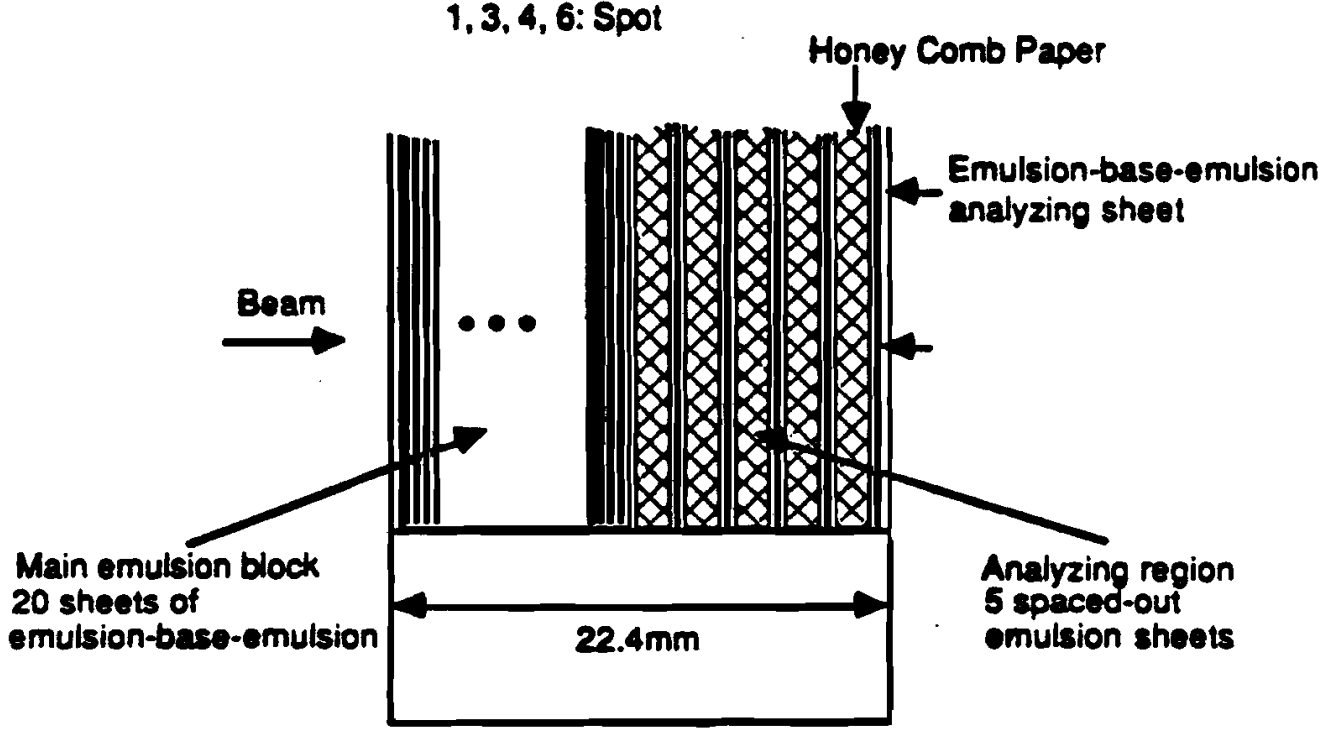

Figure 15: The configuration of a vertical emulsion module. 

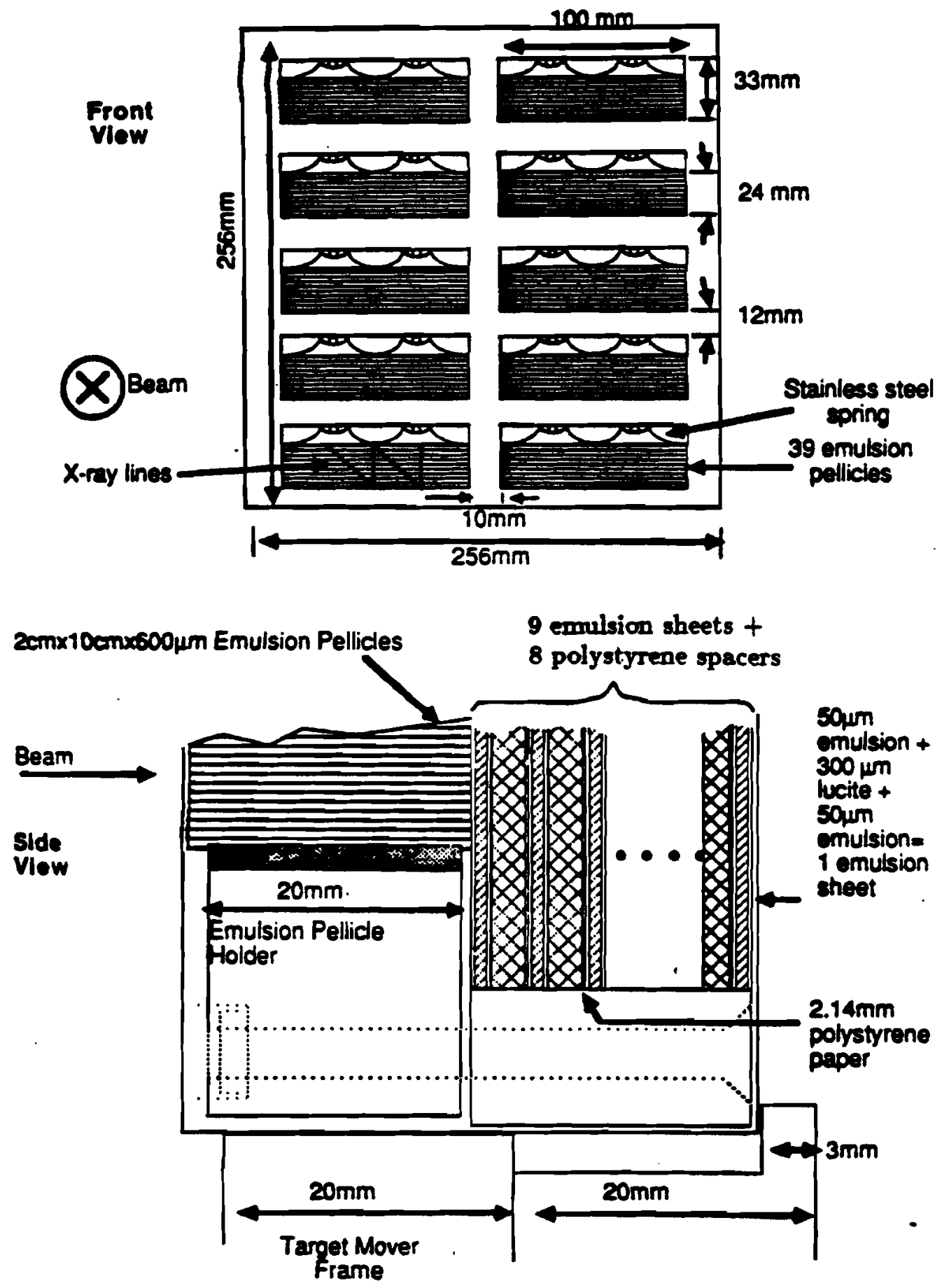

Figure 16: Horizont.al emulsion target module configiration. 


\subsubsection{Emulsion Alignment}

The exposure density in the emulsion was kept constant by moving the enulsion transverse to the beam during the beam spill. The target mover coordinates were latched and incorporated into the event information upon triggering. These coordinates were accurate to 1 micron and determined the event location.

Internal calibration of the emulsion modules and alignment to the electronic spectrometer was an important and difficult task. Upon developing the emulsion module shrinks and distorts. By controlling the developing process (uniform drying temperature) the shrinkage is kept as uniform as possible. Internal calibration was accomplished by beam spots and x-ray marks. Beam spots were exposures to the bean at known positions, by comparing the distance between beam spots before and after developing the shrinkage can be accounted for. The horizontal modules used an x-ray gun to etch lines into the pellicles. By aligning $\mathrm{x}$-ray lines the horizontal pellicles could be calibrated relatively.

Alignment to the electronic spectrometer was done by aligning beam track trajectories from the beam system to tracks in the emulsion. To aid this alignment each module was framed with a low track density region to allow this matching to proceed in a relatively clean environment. This aligmment had a precision of $\sim 10$ microns and allowed event location in the enulsion to proceed quickly. 


\subsection{Charged Particle Spectrometer}

Upon leaving the emulsion module the particles entered the first arm of the charged particle spectrometer, the vertex silicon strip detectors(VSSD's). The charged particles were bent by the magnet field of the analyzing magnet, SCM-104, before entering the spectrometer drift chambers (SDC's) that followed the magnet. This system not only provided momentum measurements for the charged tracks, it also provided vertex reconstruction. The most important being the position of the primary nuclear interaction.

The spectrometer was designed to cope with the high track multiplicity and high density of tracks in the highly collimated central region. The VSSD's achieved a two track separation of 60 microns, and the spectrometer drift chambers had two track separation of 600 microns.

\subsubsection{Vertex Silicon Strip Detectors (VSSD's)}

The VSSD's were composed of 18 planes of $5 \mathrm{~cm} \times 5 \mathrm{~cm}$ silicon strip detectors. The center to center strip distance (pitch) was 50 microns. The 18 planes were stacked $7 \mathrm{~mm}$ apart in $\mathrm{Z}$ and strips were positioned in there different projections $\mathrm{X}, \mathrm{U}$ and $\mathrm{V}$ with the $\mathrm{U}$, and $\mathrm{V}$ projections at an angle of $\pm 60^{\circ}$ with the $X$ axis. The entire stack was positioned 7 linm from the upstream face of the target emulsion and was approximately $150 \mathrm{~mm}$ in length. The $5 \mathrm{~cm} \times 5 \mathrm{~cm}$ detector furthest from the target had an acceptance of $110 \mathrm{mrad} \times 110 \mathrm{mrad}$. However, tracks with larger angles could be reconstructed in the planes closer to the target.

The 50 micron pitch and $5 \mathrm{~cm}$ wilth translates to 1000 lines per detector. To economize electronic costs and cooling needs not every line was read out. Figure 17 shows the read-out 
structure of the VSSD's. The central 7.95mm of the VSSD's had every line instrumented, every other line was read out in the next $3.6 \mathrm{~mm}$. Every third line was read out $7.575 \mathrm{~mm}$ from the center strip to $9.975 \mathrm{~mm}$. The outer $15 \mathrm{~mm}$ had every fifth line instrumented. Capacitive coupling between adjacent strips permitted a charge sharing algorithm to achieve a spatial resolution better than the instrumented strip separation alone would give. The amount of charge deposited in each strip was recorded and the amount of charge determined how far from the instrumented strip the particle hit the detector. The residuals for the four different read out regions are shown in Figure 18 and the resolution for the central region was measured to be $8.8 \mu \mathrm{m}$ rms. The decision to read out the pulse height from each strip, instead of just recording whether or not the strip had activity $(0 / 1)$ meant that 7000 lines of VSSD information had to be digitally converted and read out by the data acquisition system.

The detectors had $96 \%$ efficiency during the run and required very little maintenance.

\subsubsection{Analyzing Magnet}

The charged particles were bent by the magnet field of the SCM-104 analyzing magnet. The gap separation was $50.8 \mathrm{~cm}$ with shaped pole pieces to increase the magnetic field without losing any acceptance. This asymmetric shape, shown in Figure 19, resulted in a non-uniform field that had symmetry in the $\mathrm{Y}-\mathrm{Z}$ plane only.

The field was mapped out in intervals of $12.7 \mathrm{~mm}$ in $\Delta \mathrm{Z}$ and $25.4 \mathrm{~mm}$ in $\Delta X$ and $\Delta Y$. 


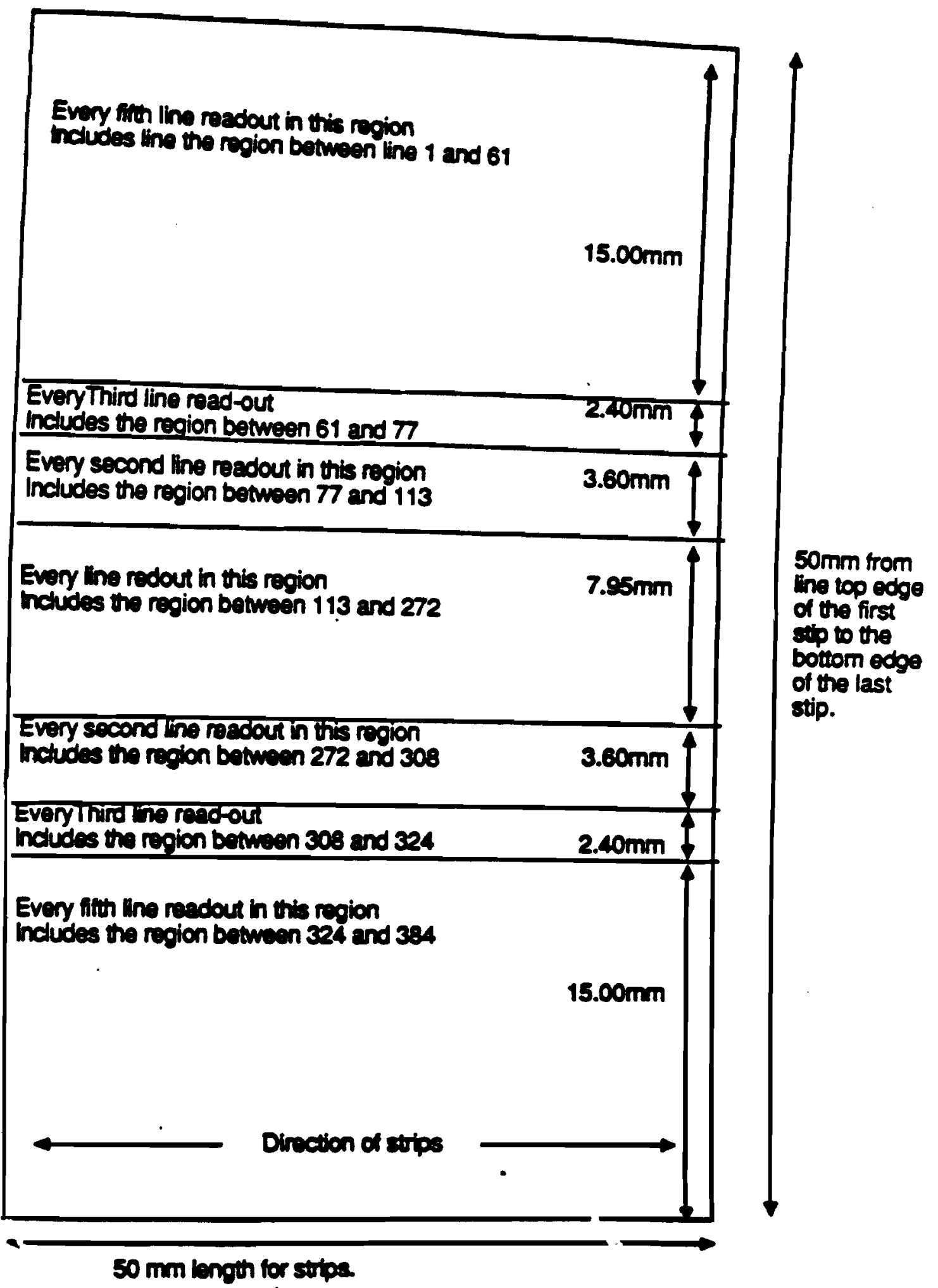

Figure 17: Read out pattern for the vertex silicon strip detectors. 


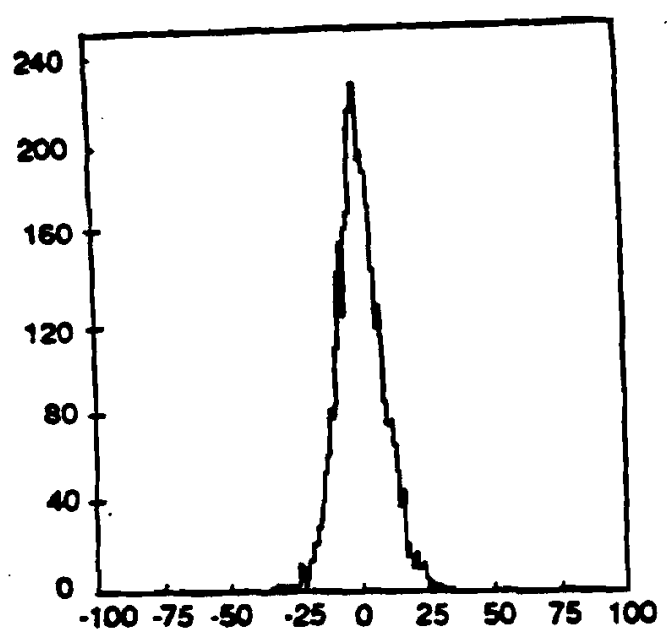

Every line readout region

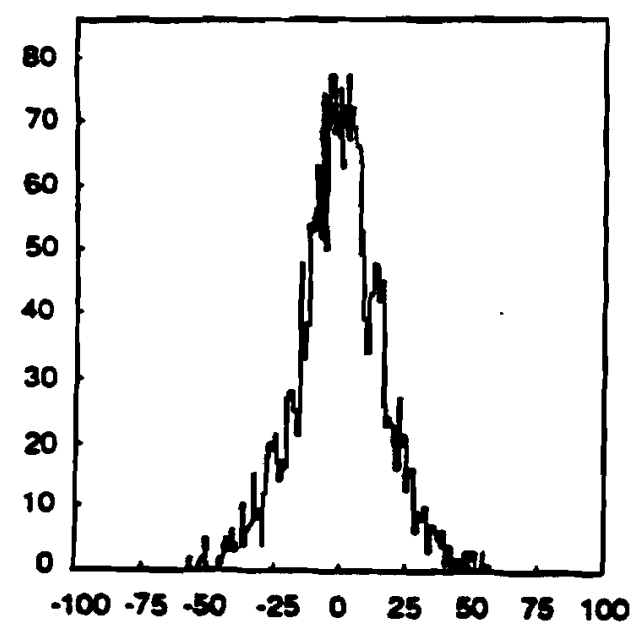

Every third line readout region

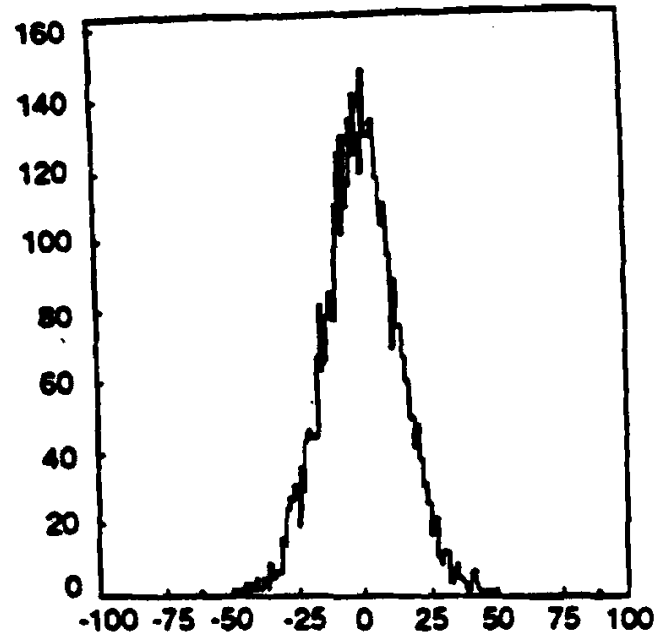

Every two line readout region

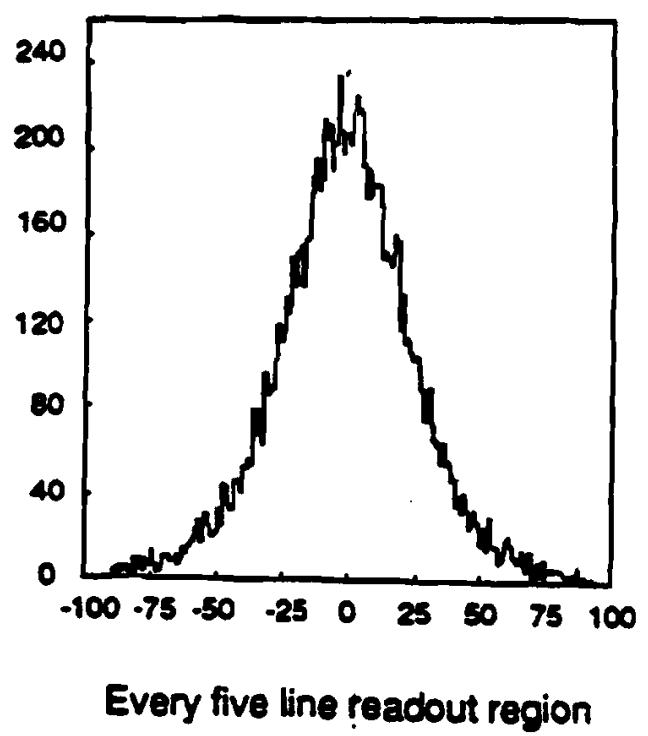

Figure 18: Residunls in the vertex silicon strip detectors. 


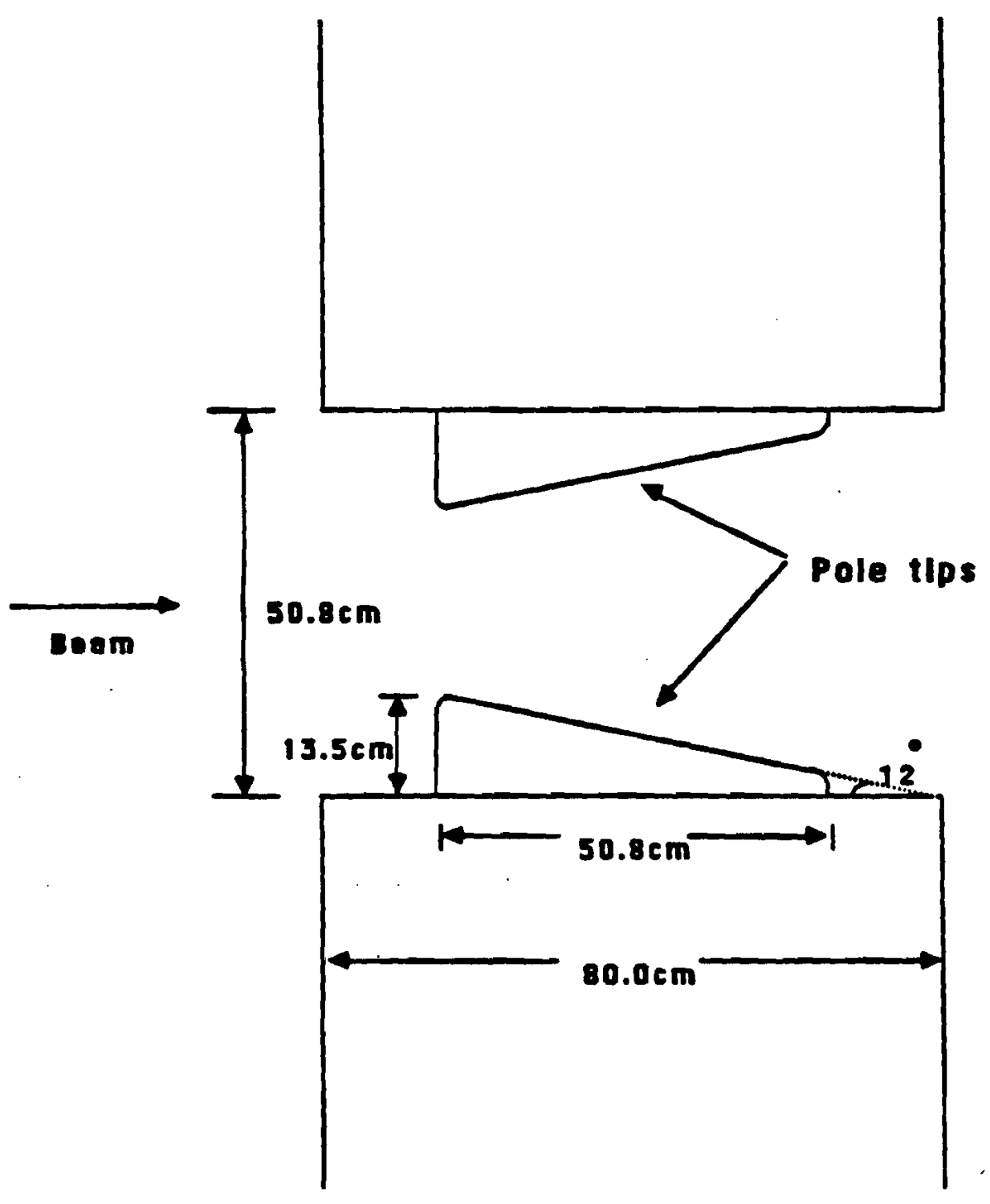

Seale: $1 / 8$

Figure 19: The poles of the analyzing magnet, SCM-104. 
The integrate field strength at 2400 amps was

$$
\int B \cdot d l=11.2 \text { kilogauss } \cdot \text { meters }
$$

The magnet imparted a transverse kick of $0.336 \mathrm{GeV} / \mathrm{c}$ to the charged particles.

\subsubsection{Spectrometer Drift Chambers}

Completing the charged particle system were 10 multi-sampling "vector" drift chambers located down stream of the analyzing magnet. The chambers covered a $1.7 \times 1.7 \mathrm{~m}^{2}$ area transverse to the beam and were in there different projections $X, U$ and $V$, with the $U$ and $\mathrm{V}$ projections rotated $\pm 30^{\circ}$ with respect to the $\mathrm{X}$ axis. The term multisampling comes from the 5 position samplings (at $1.02 \mathrm{~cm}$ spacings) along the $\mathrm{Z}$-axis per cliamber. Transverse to the beam axis the chambers were divided into 22 cells. The central 10 cells were called 'fine' cells and had a drift distance of $2.54 \mathrm{~cm}$ with sense wires every $5.1 \mathrm{~mm}$ along the $z$ direction. Surrounding the fine cells were 6 'coarse' cells. The coarse cell were iclentical to the fine cell except the sense wire spacing was twice that of the fine cells. Figure 20 shows the wire positions for the two types of cells. The cliambers were operated a 1 atmospliere of pressure and a $50 \%$ argon, $49.5 \%$ ethane and $0.5 \%$ ethanol gas nuxture.

Figure 21 shows the position residuals for the SDC's. When a single beam track passed through the chambers, the resolution was 110 microns rms. For typical secondary tracks the resolution was 140 microns. 

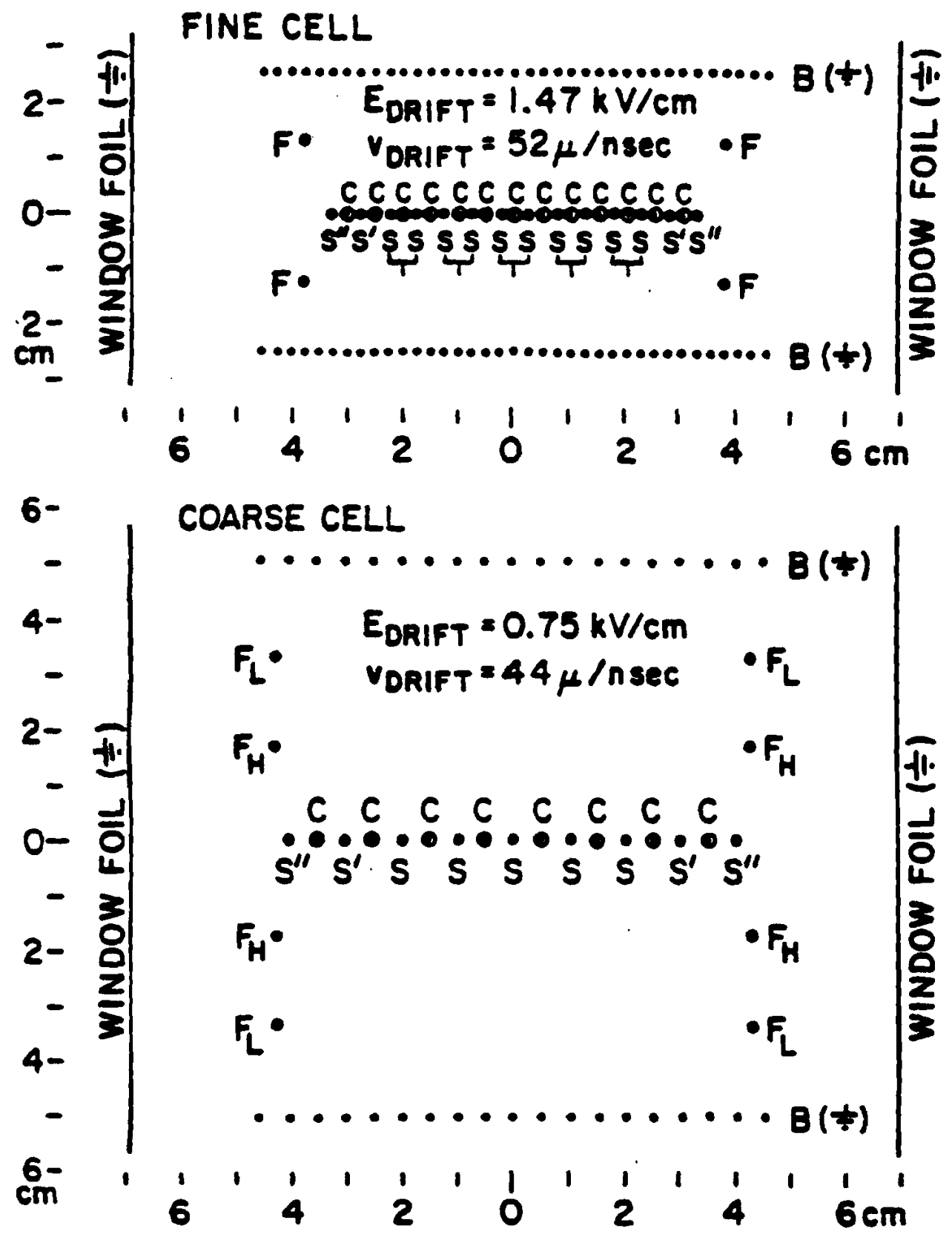

Figure 20: Wiring diagram for the fine and coarse cells in the spectrometer drift chambers. 


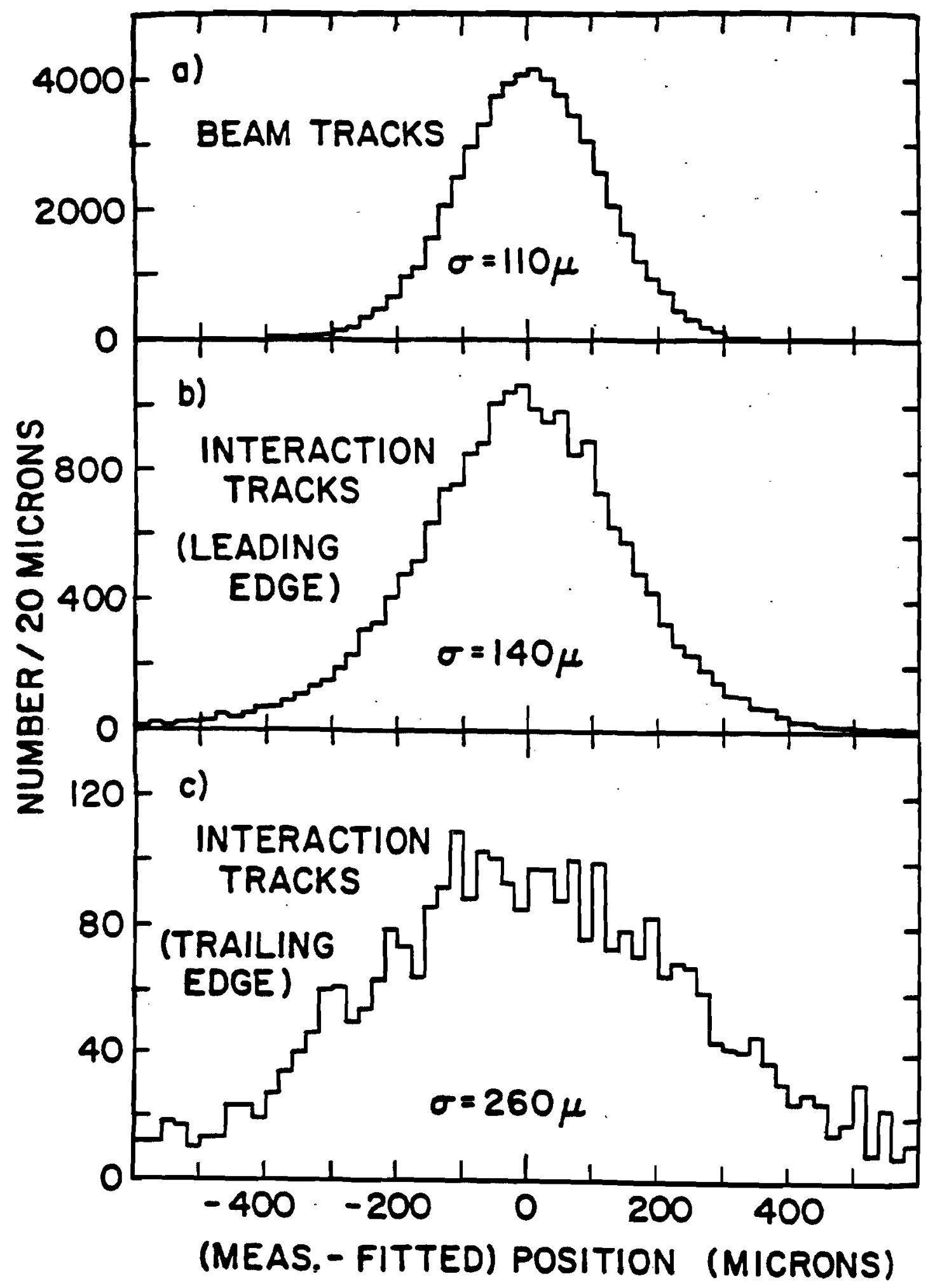

Figure 21: Position resirluals for the spectrometer drift chambers. 


\subsubsection{Momentum}

The charged particle spectrometer provided momentum measurements for tracks that were linked between the VSSD's and SDC's. For charm selection a simple thin lens approximation was used to determine the momentum. The error in this calculation was:

$$
\delta p / p=\sqrt{(0.01)^{2}+(0.00023 p)^{2}} \quad p \text { in } G e V / c
$$

The first term is from multiple scattering and second term is the contribution from slope measurement errors. An attempt to incorporate a Runge-Kutta iterative integrated fit to the track trajectories through the magnetic field found no significant improvement over the thin lens approximation [31]. The thin lens momentum estimate was used throughout this analysis.

\subsection{Muon System}

The muon system provided the only particle identification used in this analysis. Muons were tagged by scintillation counters placed down stream of 26 interaction lengths of material. The muon hodoscope was preceded by the muon spectrometer which provided an independent measurement of the muon trajectory and momentum. The muon spectrometer consisted of two sets of. 6 drift chambers and a toroidal magnet. Figure 22 shows the plan view of the complete muon system. 

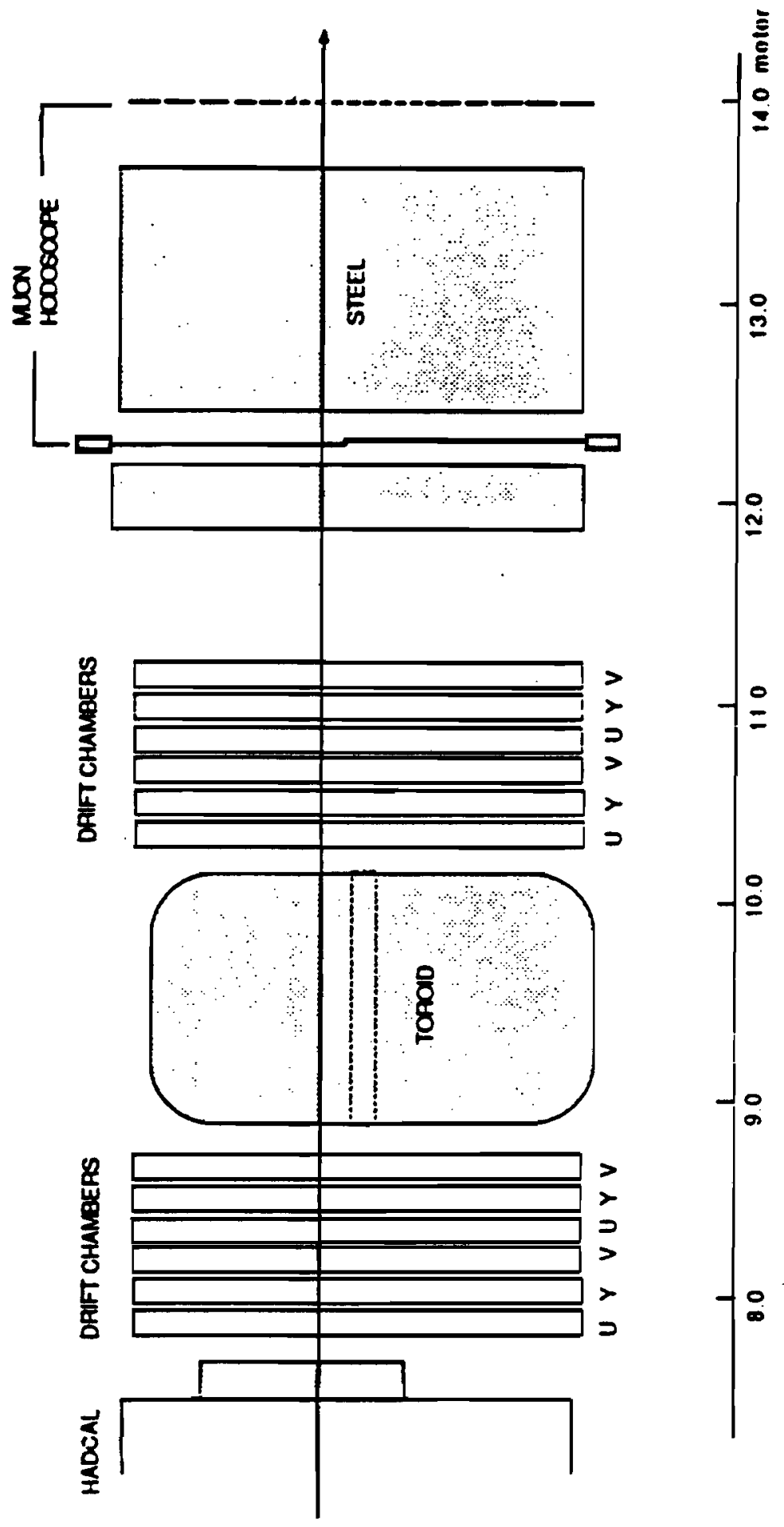

Figure 22: Plan view of the muon system. 


\subsubsection{Muon Spectrometer}

After leaving the SDC's the particles encountered the time of flight counters, liquid argon calorimeter, hadron calorimeter, and ranging steel. The information provided by these systems was not used in this analysis. Particles that managed to get through the 8.77 interaction lengths of material in the calorimeters entered the muon spectrometer.

The muon spectrometer consisted of 12 drift chambers with a $3 \mathrm{~m} \times 3 \mathrm{~m}$ area transverse to the beam axis. The chambers were arranged in three views, $Y, U$ and $V$. The $U$ and $\mathrm{V}$ views were set at $\pm 12^{\circ}$ with respect to the $\mathrm{X}$-axis. Each chamber had two $1.6 \mathrm{~cm}$ wide gaps, and the sense wires in the two gaps were offset to eliminate left/right ambiguity. Six chambers preceded the steel toroid magnet, representing 8.03 interaction lengths, and six chambers followed the magnet. The last drift chamber was just $11 \mathrm{~m}$ from the emulsion target.

The chambers were operated initially with $50 \%$ argon $49.5 \%$ ethane and $0.5 \%$ ethanol gas mixture, for the later runs a $50 \%$ argon $50 \%$ ethane gas mixture was used.

Unexpected non-uniform electric fields caused by floating some of the shaping strips caused a non-linear drift velocity. The non-linear effects were determined by using a high momentum muon calibration beam. The final overall position resolution of 400 microns was achieved when properly accounting for the non-linear velocity on a cell by cell basis. Figure 23 plots the residuals for four of the muon drift chambers. 

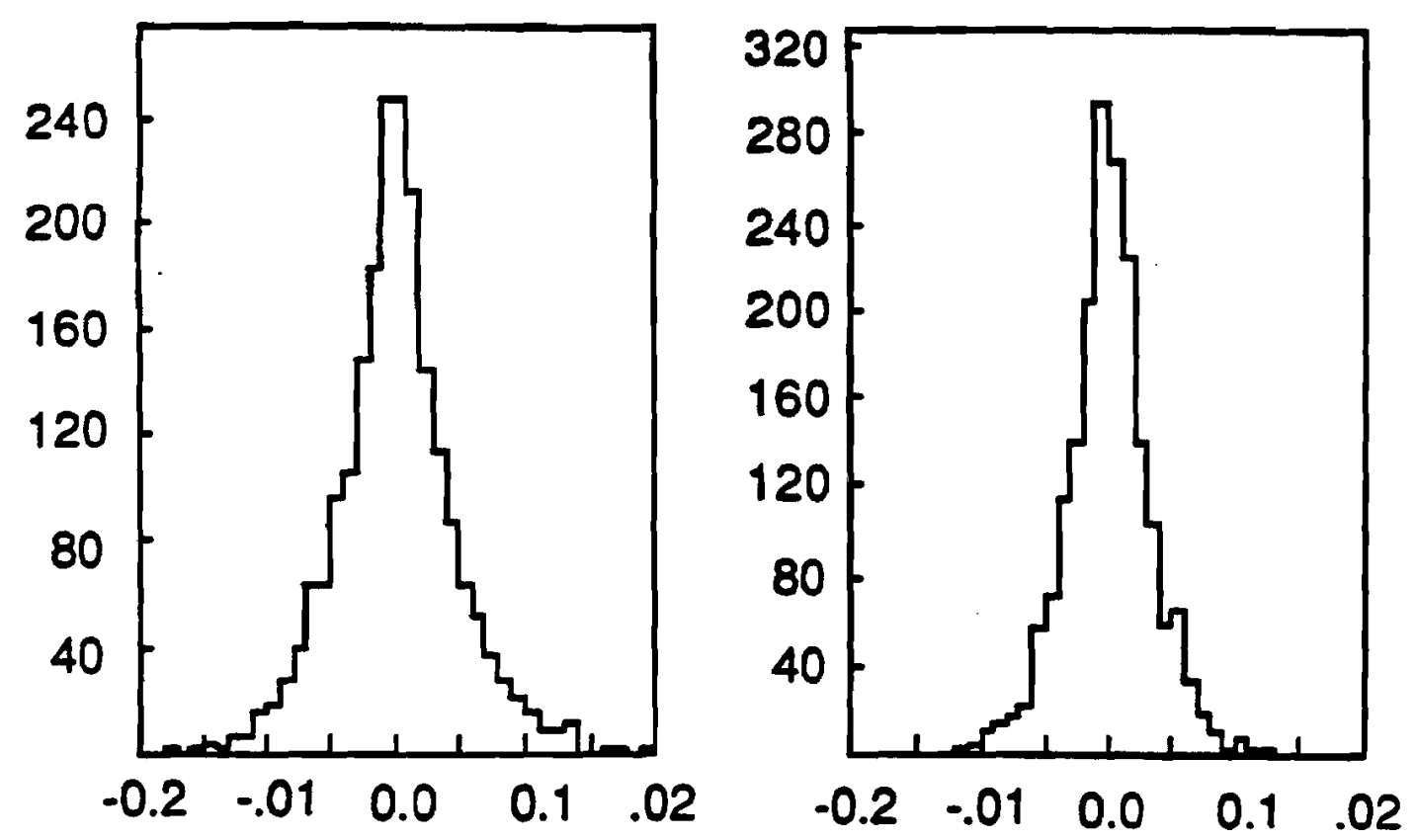

CHAMBER 1 FRONT RESIDUALS
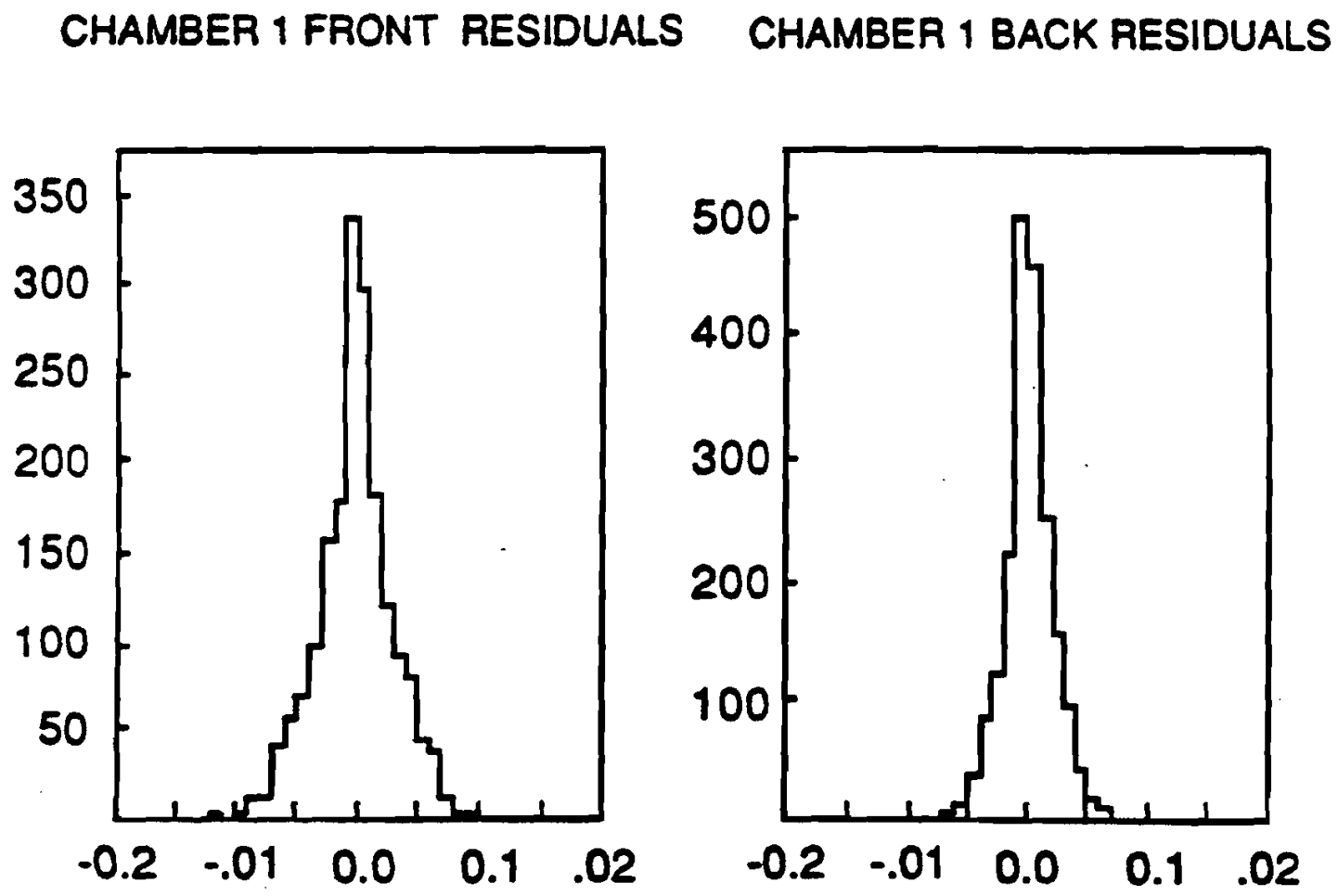

CHAMBER 2 FRONT RESIDUALS CHAMBER 2 BACK RESIDUALS

Figure 23: Position residuals for four of the cells in the muon drift chambers. 


\subsubsection{The Toroid}

Sandwiched between the muon drift chambers was a toroidal steel magnet, $130 \mathrm{~cm}$ thick and a radius of $180 \mathrm{~cm}$. The toroid consisted 8.03 interaction lengths of steel. In conjunction with tracking information provided by the muon drift chambers, the toroid provided an independent momentum measurement of the muon momentum. This momentum provided another constraint on the linkage of the muon track reconstructed by the muon system to its upstrean track in the spectrometer drift chambers.

The field map of the toroid was generated with the POISSON program in the CERN program library. The results of the program agreed within $2 \%$ with hall probe measurements. The equation for the magnetic field of the toriod is:

$$
\begin{gathered}
B(r)=22.196-0.040049 r \quad 12<r<86 \\
B(r)=21.037-0.024904 r 86<r<775
\end{gathered}
$$

The momentum equation is:

$$
P=0.03 B(r) L / \theta
$$

The resolution is:

$$
\delta P / P=\sqrt{(0.19)^{2}+(0.007 p)^{2}}
$$

- $r$ = radius from center of magiet $(\mathrm{cm})$ 
- $\mathrm{L}=$ thickness traversed $(\mathrm{cm})$

- $\theta=$ angle deflection of the track (mradians)

- $\mathrm{P}=$ momentum in $\mathrm{GeV} / \mathrm{c}$

The magnetic field in the toroid was $19.5 \pm 2.5$ kilogauss and imparted a $0.76 \mathrm{GeV} / \mathrm{c}$ momentum kick to the muons.

The amount of ranging material preceding the muon system was not quite enough to contain the hadronic cascade caused by the incident hadrons. This hadron punch through swamped the six upstream muon drift chambers. The muon drift chambers were designed to operate in a low multiplicity environment. The front muon chambers were incapable of resolving the muon track in events with large hadron punch through.

Because of the hadronic punch through the events were divided into three types:

- Up-Down Muons: The muon track was successfully reconstructed in both sets of muon drift chambers. $57 \%$ of the events.

- Down Only Muons: Muon track information is available from the downstream chambers only. $38 \%$ of the events.

- No Muon: No muon track in the downstream chambers, $5 \%$ of the events. These events were later rejected by the offline analysis.

\subsubsection{Muon Hodoscope}

At the very end of the E653 spectrometer, $15 \mathrm{~m}$ from the emulsion target, was the muon hodoscope. The muon hodoscope consisted of two sets of scintillation counters seperated 
by ranging steel. The effective area of the counters was $360 \mathrm{~cm} \times 304 \mathrm{~cm}$ and had an angular acceptance of +99 to -157 milliradians in $d x / d z$. And +142 to -99 milliradians in $d y / d z$. The front scintillators, $\mathrm{MU}_{\text {Front }}$ consisted of 36 horizontal counters logically 'or'ed together. The 40 back paddles were oriented vertical behind another 6.67 interaction lengths of steel and were also logically 'or'ed together. A particle was tagged as a muon if both $\mathrm{MU}_{\text {Front }}$ and $\mathrm{MU}_{\text {Back }}$ arrays fired in coincidence.

$$
M U=\Sigma M U_{\text {Front }} \cdot \Sigma M U_{\text {Back }}
$$

A particle had to traverse 26 interaction lengths of material to reach the $M U_{B \text { ack }}$ paddles.

The signal in the MU hodoscope was used to complete the trigger requirements discussed in the next section.

The efficiency of the muon hodoscope was $94 \%$. This was determined by removing the muon requirement from the trigger. The number of events with both a muon track reconstructed in the MDC and a hit registering in the hodoscope was compared to the number of events with a reconstructed muon track.

\subsection{The Trigger}

The E653 trigger was the logical 'and' of three scintillator counter systems, the beam trigger counters, the interaction counter and the muon hodoscope. The beam trigger counters and the interaction counter are shown in Figure 24. The beam cuunters forced the incoming proton to be incident on the central regions of the silicon strip detectors and required that 
the proton was not associated with any halo particles. The counter $S_{3}$ which was $5 \mathrm{~mm}$ in diameter defined the beam position and also provided the start time for the time to digital converters discussed in the next chapter. The interaction counter was placed immediately after the emulsion stack and was $6.35 \mathrm{~mm}$ thick. The signal from this counter was sent to a discriminator with the threshold set at the equivalent of 3 minimum ionizing particles. This counter required that an interaction had occurred in the emulsion, and produced at least three charged tracks.

The muon hodoscope, described in the previous section, completed the trigger.

The logical definition of the E653 trigger was

$$
\text { Trigger }=\text { Beam } \cdot \text { Interaction } \cdot \text { Muon }
$$

This was referred to as the $B \cdot I \cdot \mu$ data trigger.

The data trigger was vetoed by the data acquisition system if it was busy reading out a previous event or if the nuemory had insufficient space available for another event. The dead time was 25 to $30 \%$. Figure 25 shows the logical schematic of the Run I E653 trigger.

\subsection{Data Acquisition}

The jub of the data acquisition system was to convert the analog information into binary numbers, digitization. This information must then be "packed" into a readable format and transcrilsed to a recording medium, magnetic tape or hard disk. The data acquisition 


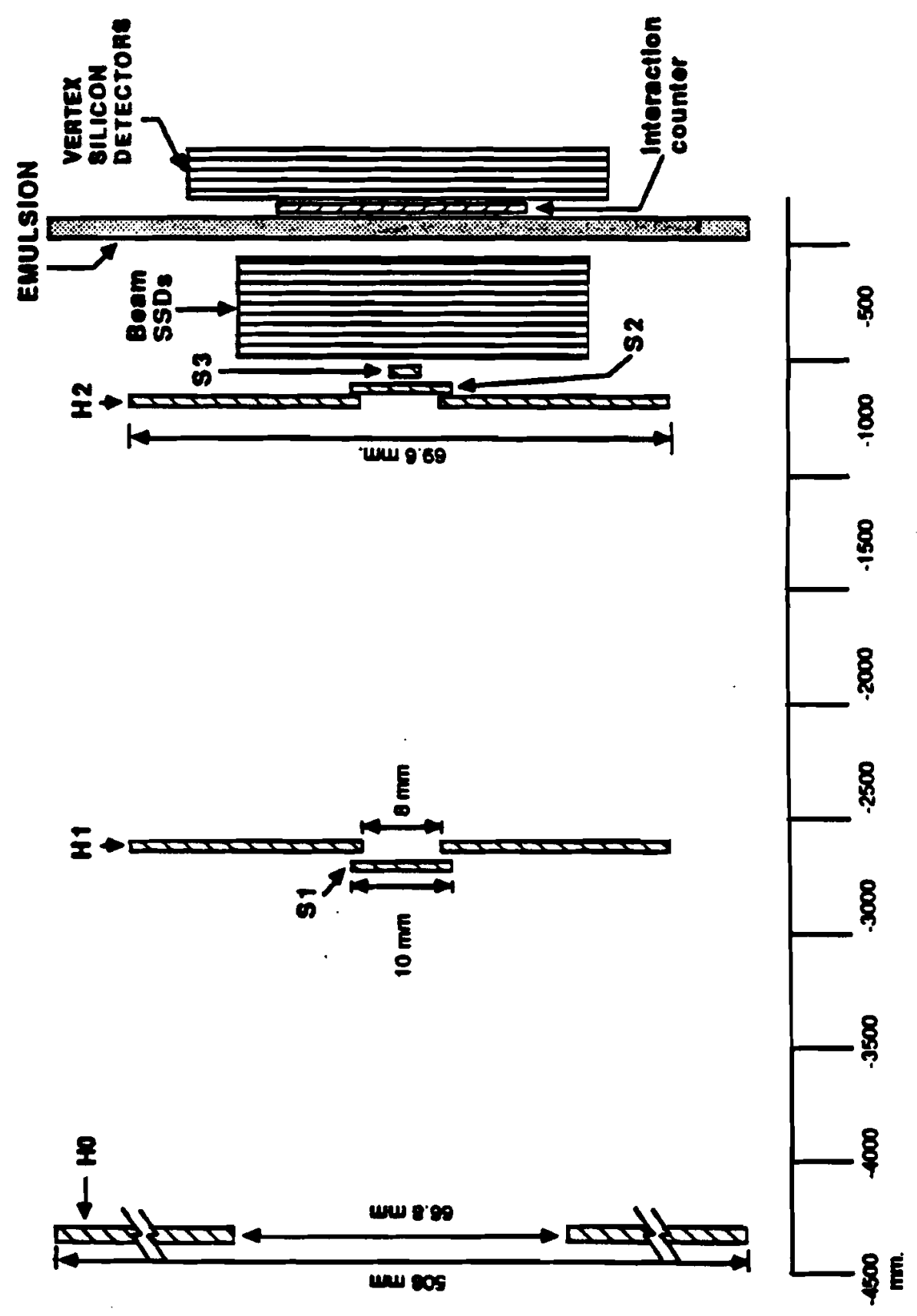

Figure 24: The beam and interactions counters of the E653 trigger system. 


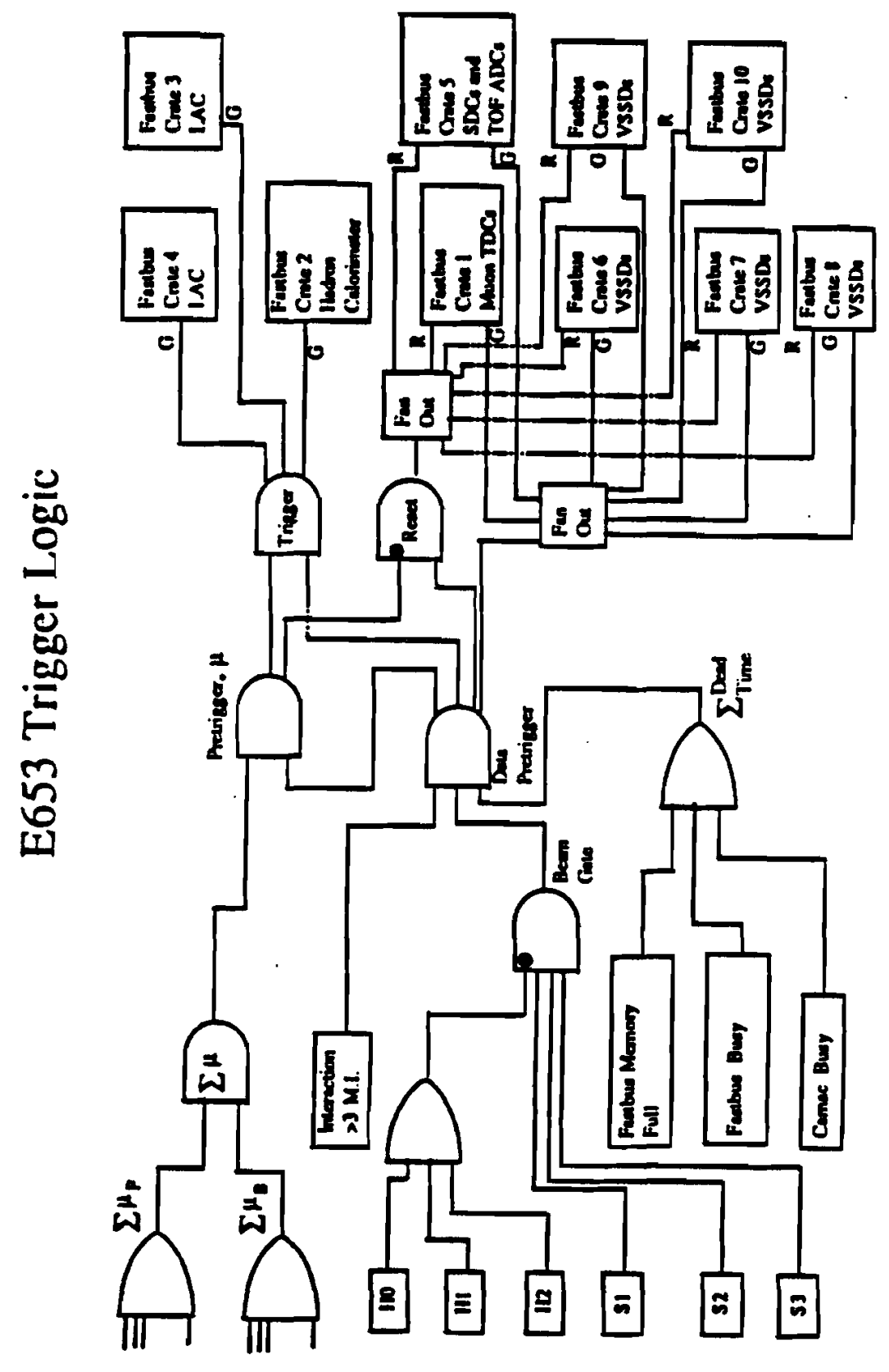

Figure 25: Logical schematic of the E653 trigger. 
system used by E653 was the new FASTBUS data acquisition system.

E653 was the first experiment to use the new FASTBUS data acquisition system. Being the first meant that the majority of the devices had to be developed and debugged on the experimental floor. For example: a direct path connecting the FASTBUS system to a VAX computer architecture did not exist at the time of the first data run (1985). For this reason a rather convoluted FASTBUS to LSI 11/83 to VAX 11/750 data path had to be used. This data path and the inclusion of some CAMAC modules, because the FASTBUS equivalents were unavailable at the time, slowed the data rate to almost the same rate as the older CAMAC system. The compactness of FASTBUS modules compared to the older CAMAC modules made up for lack of a significant improvement in the data rate. This section will briefly review the data acquisition system (DA) and the ONLINE programs used to monitor the performance of the equipment during data taking.

\subsubsection{Fastbus Hardware}

The number of lines of information per experiment grows as the cost of associated electronics becomes tolerable. The problem becomes one of increasing the density of information. With this in mind the FASTBUS data acquisition system was developed. The FASTBUS system is a modular and crate system. Each crate would hold a programmable unit that controlled the crate and the data acquisition modules. Each data acquisition module had 96 channels of input and each crate could hold up to 22 data acquisition modules. The FASTBUS modules used were the first generation modules produced by Lecroy Research Services.

The Lecroy 1885 anolog to digital converter ( $\mathrm{ADC}$ 's) used by the silicon strip detectors 
and calorimeters had bi-level readout capability. In the low range the sensitivity was 50 femtocoulombs/count and the high range sensitivity was 400 fentocoulombs/count. The total range of the ADC's was 200 picocoulombs for the low range and 1600 picocoulombs for the ligh range. Each $A D C$ received a gate from the crate Calibration and Trigger Module (CAT). The timing and width of the gate was set by the experimenter.

The Lecroy 1879 time to digital converters (TDC's) were used by all the drift chamber systems to convert the time difference between the start pulse from trigger counter $S_{3}$ and the leading and trailing edges of the signal pulse from the drift chambers. The resolution of the TDC's depended on the clock frequency. The clock frequency was provided by the CAT module and was set at $334 \mathrm{MHz}$ and had a bin width of 3 nanoseconds.

In addition to performing the digitization of the event information each FASTBUS crate had a CAT module which provided the ADC's and TDC's with the timing pulses (gates, and start and stop times). Each crate also had a programmable processor module (Lecroy 1821) that enabled online pedestal subtraction of the ADC signals. The digitized information from each crate was sent to its memory module, the Lecroy 1891. The memory module had multiple record capalilities and could hold up to one megabyte of information (two of the 1891's had four megalyyte capabilities).

Once the event made it into the FASTBUS memory buffer the information was slipped to a LSI $11 / 73$ via the DR11W interface. From there the information was shipped to the online computer, a VAX $11 / 750$, and then onto magnetic tape. The online data acquisition system ruming on the VAX skimmed some of the events onto disk for online monitoring of the apparatus. The complete online data acquisition system data path is shown in Figure 
26. With this configuration $\mathbf{E} 653$ was able to achieve a data rate of 122 Kilobytes/sec.

\subsection{The 1985 Run and Overall Performance}

The first rum of the spectrometer just described started in May 1985 and was over by August of that year. The spectrometer had its quirks during this rumning period but for the most part the problems in keeping the hardware rumning were inconsequential to its final performance. Figure 27 shows the number of B-I. $\mu$ triggers written to tape during the 1985 rum. Periods of down time were due to lack of beam to the experiment, not equipement failure.

After the data taking run the individual groups responsible for building the detectors attacked the problem of writing the software needed to do the event reconstruction. Various calibration data were taken during the run:

- High momentum $\mu$ tracks were used to calibrate and align the muon spectrometer, spectrometer drift chambers and vertex silicon strip detectors.

- Low momentum electron beams were used to calibrate the liquid argon calorimeter.

- Low momentum hadrons and ligh momentum nuons were used to calibrate the hadron calorimeter.

- Conversion electrons from a parasitic $\gamma$ beam were used to provide low momentum electrons used for additional LAC calibration.

- $800 \mathrm{GeV} / \mathrm{c}$ protons, no interaction target, were used to align the beam system, VSSD's, 


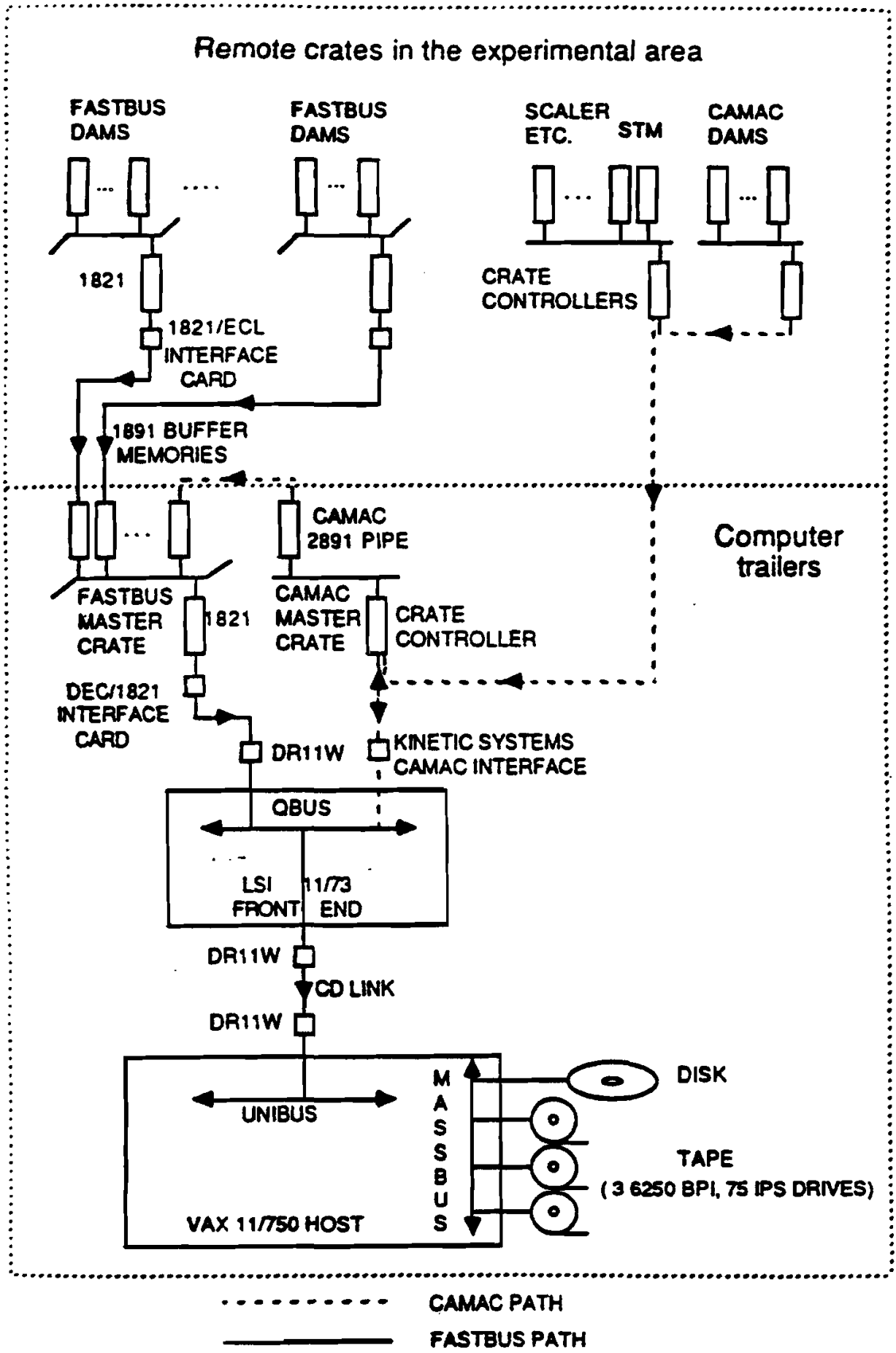

Figure 26: The event data path from the the FASTBUS crates to the magnetic tape. 


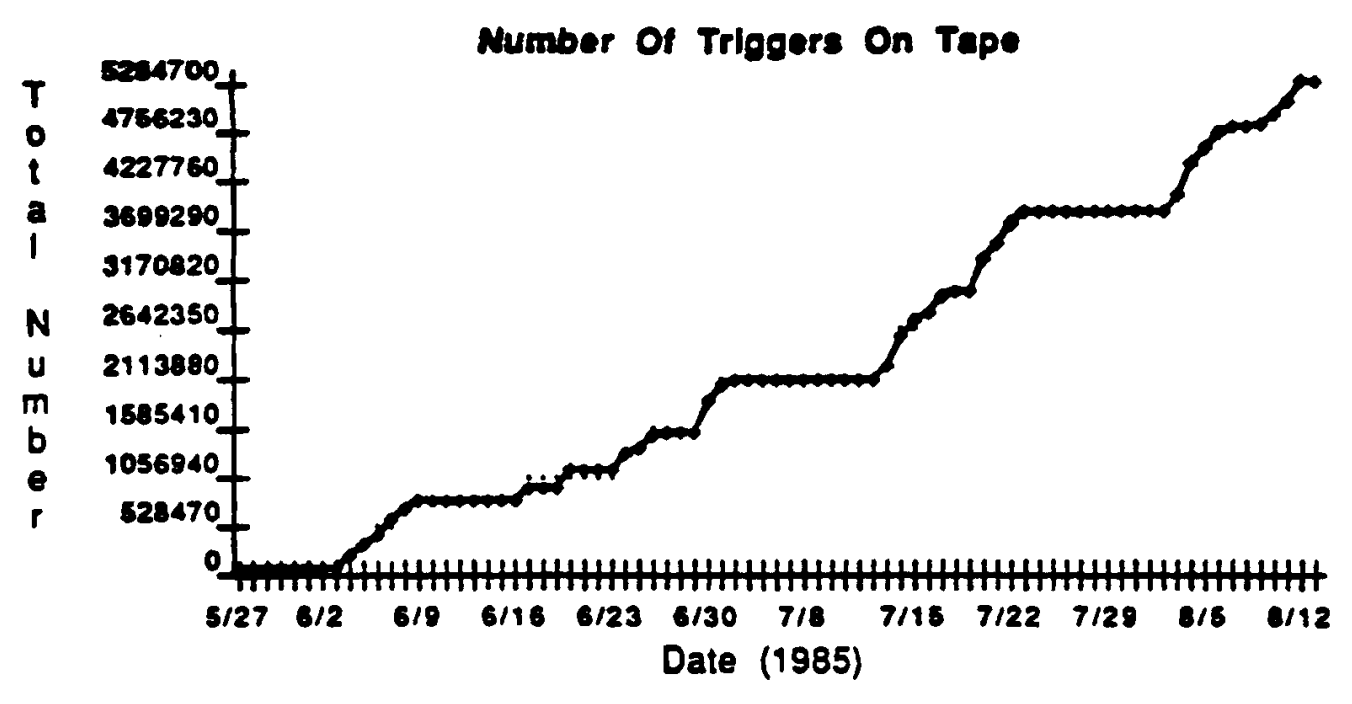

Figure 27: The integrated number of data triggers written to tape during the 1985 run of E653.

and SDC's.

$B \cdot I \cdot \mu$ data triggers were also used by the system for additional alignment and calibration.

The resolution achieved by the individual systems is listed in Table 5 . 


\begin{tabular}{|c|c|c|c|}
\hline System & Quantity & Units & Resolution \\
\hline $\begin{array}{l}\text { Beam Drift } \\
\text { Chambers }\end{array}$ & Position/plane & $\mu \mathrm{mm}$ & 150 \\
\hline $\begin{array}{l}\text { Beam Silicon } \\
\text { Strips }\end{array}$ & Position/plane & $\mu \mathbf{m}$ & $17-35$ \\
\hline Combined & Position at $\mathrm{z}=0$ & $\mu \mathrm{m}$ & 10 \\
\hline Beam System & Angular & $\mu \mathrm{rad}$ & 20 \\
\hline Ennulsion & $\begin{array}{l}\text { Position } \\
\text { Angular }\end{array}$ & $\begin{array}{c}\mu \mathrm{m} \\
\text { milliradiains }\end{array}$ & $\begin{array}{l}1-10 \\
1-10\end{array}$ \\
\hline Vertex SSD & $\begin{array}{c}\text { Position/plane } \\
\text { Position at } z=0 \\
\text { Angular }\end{array}$ & $\begin{array}{c}\mu \mathrm{m} \\
\mu \mathrm{m} \\
\mu \mathrm{rad}\end{array}$ & $\begin{array}{c}8-24 \\
10 \\
70 \\
\end{array}$ \\
\hline $\begin{array}{l}\text { Spectrometer } \\
\text { Drift Chambers }\end{array}$ & $\begin{array}{c}\text { Position/plane } \\
\text { Angular }\end{array}$ & $\begin{array}{c}\mu \mathrm{m} \\
\mu \mathrm{rad}\end{array}$ & $\begin{array}{c}50-60 \\
35\end{array}$ \\
\hline $\begin{array}{l}\text { Primary Vertex } \\
\text { Secondary Vertex }\end{array}$ & $\begin{array}{c}\text { Position(z) } \\
\text { Position }(x, y) \\
\text { Position }(z) \\
\text { Position }(x, y)\end{array}$ & $\begin{array}{l}\mu \mathrm{mm} \\
\mu \mathrm{m} \\
\mu \mathrm{m} \\
\mu \mathrm{m}\end{array}$ & $\begin{array}{c}300-400 \\
6-10 \\
550-750 \\
11-18\end{array}$ \\
\hline$\overline{\mathrm{LAC}}$ & $\begin{array}{l}\text { Position(x,y) } \\
\text { Energy }(\delta E / E)\end{array}$ & $\underset{\%}{\mathrm{~mm}}$ & $\begin{array}{c}1.2 \\
(12 / \sqrt{E})+2.5\end{array}$ \\
\hline $\begin{array}{l}\text { Muon Drift } \\
\text { Chambers }\end{array}$ & Position $(x, y)$ & $\mu \mathrm{m}$ & $250-600$ \\
\hline $\begin{array}{l}\text { Charged Particle } \\
\text { Spectrometer }\end{array}$ & Momentum & $\begin{array}{c}p \text { in } \\
\mathrm{GeV} / \mathrm{c}\end{array}$ & $\sqrt{(0.01)^{2}+(0.00023 p)^{2}}$ \\
\hline Muon Spectrometer & Momentum & $\mathrm{p}$ in $\mathrm{GeV} / \mathrm{c}$ & $\sqrt{(0.19)^{2}+(0.007 p)^{2}}$ \\
\hline
\end{tabular}

Table 5: The individual detector resolutions for the E653 spectrometer. 


\section{Chapter 4}

\section{Charm Selection}

The motivation behind the E653 spectrometer was to collect a large number of unbiased heavy quark decays located in an emulsion target, by triggering on the semi-muonic decay of the partner quark. Emulsion scanning candidates were selected via offline spectrometer reconstruction. These events were visually scanned in the emulsion and decays with a topology consistent with charm were saved as charm candidates. The topological candidates went through a second pass of spectrometer reconstruction and decays consistent with charm decays were used for production analysis. This chapter describes the complete analysis chain used to obtain the charm pair sample, starting with the initial muon trigger.

\subsection{E653 Trigger}

Semi-leptonic decay rates of heavy quarks were predicted by theory to be lai 0 ? at the time E653 was being designed. Measurements just being made of this important decay mode 
for charm mesons agreed with the predicted rates $[25,63]$. Hadronic interactions produce a charm event for every 1000 interactions, the muonic trigger enhanced the charm yield by eliminating background interactions that did not produce a high momentum muon.

E653 used a muon hodoscope positioned behind $5-\mathrm{GeV}$ of ranging steel to trigger the data acquisition system (see Figure 22). The trigger also required a well defined beam particle and at least three charged particles leaving the emulsion (see Figure 24). These three requirements together formed (in E653 jargon) the B.I. $\mu$ trigger where the B stands for beam, I for interaction and $\mu$ for $\mu$-hodoscope. The results for this work are from the $5 * 10^{6}$ B.I. $\mu$ triggers written to tape and the 35 liters of exposed emulsion during the 1985 run.

\subsection{Event Reconstruction and Emulsion Predictions}

The first stage of the offline analysis used the tracking information in the VSSD, SDC, and MDC to select charm events and to determine the location of the events in the emulsion block. Charm selection utilized the properties of the reconstructed trigger muon track; event location was determined by the reconstructed primary vertex position.

The trigger muon track had to be reconstructed in the muon drift chambers and it had to be linked to a track in the upstream tracking detectors. The linking was done at the mid-plane of the material between the SDC and the MDC systems. At this mid plane $(Z=$ $710 \mathrm{~cm}$ ) multiple scattering effects are mininuzed. The momentum measurement of the muon track in the MDC system and the upstream spectrometer had to agree in sign and 


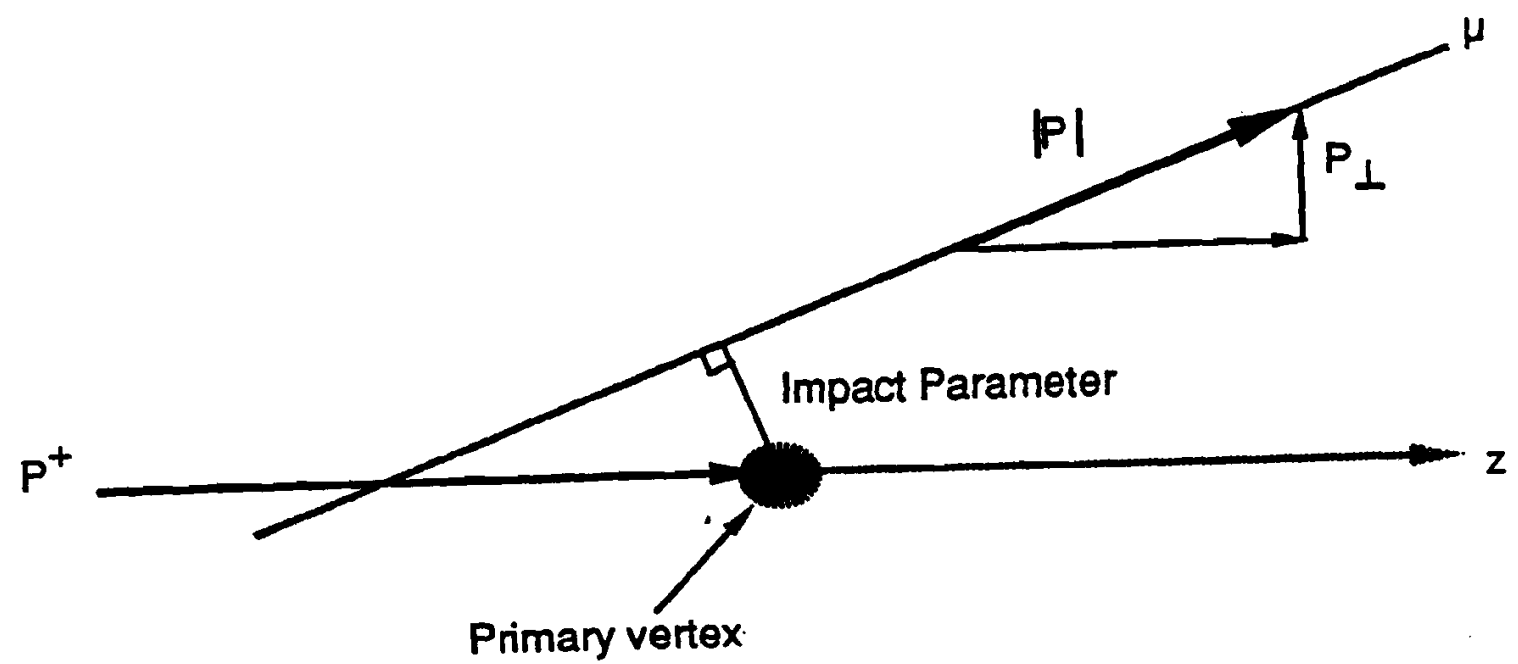

Figure 28: Cut variables applied the muon track to select events for emulsion scanning.

value. Muon tracks were required to have a $\chi^{2}<3.0$ for the link between the MDC and the SDC, and $\chi^{2}<5.0$ for the link between the SDC and VSSD. The linking efficiency between the MDC and SDC was $35 \%$. The major source of the linking inefficiency was due to $\mathrm{K}$ or $\pi$ decay into muons and the unexpectedly large amount of haclron punch through in the first six muon drift chambers. The linking requirements insured that the event contained a high quality reconstructed trigger muon and allowed the properties of the muon track to be used for event selection. Approximately, 35\% of the events had a completely reconstructed trigger muon track.

The reconstructed tracks in the VSSD's that linked to the SDC's (so they had a monnentum measurement) were used to form primary and secondary vertices. If a primary vertex was reconstructed inside the main enulsion block, the impact parameter of the muon with respect to the primary vertex position was deternined (see Fig. 28). 
The charm selection cuts used in this analysis are listed in Table 6 . There were four sets of cuts involving several variables, but the most significant cuts involved the impact parameter of the $\mu\left(b_{\mu}\right)$, the transverse momentum $\left(P_{\perp}\right)$ of the $\mu$ with respect to the beam direction and a momentum cut on the muon $\left(P_{\mu}\right)$. The momentum cut $\left(P_{\mu}>8.0 \mathrm{GeV} / \mathrm{c}\right)$ on the muon track insured a high momentum muon which reduced the effects of multiple scattering in the emulsion and the in the ranging steel. The $\mathrm{P}_{\perp}$ cut reduced the background from $\mathrm{K}$ or $\pi$ decay into muons, only $5 \%$ of muons from prompt $\pi$ 's decays survived the cut while $40 \%$ of muonic charm decays survived [63]. This is due to the combination of a larger $P_{t}$ available in charm decay and the fact that charm is produced with a higher average $P_{\perp}$. Kaons and pions from charm decay which subsequently decay into muons were not eliminated by this cut. This sample of mis-identified muonic charm decays represents about $14 \%$ of the muonic sample [62]. The impact parameter cut insured that the muon was not associated with primary interaction.

The three different classes of cuts were established with the nuon cut variables $\left(\mathrm{P}_{\mu}\right.$, $\left.b_{\mu}, P_{\perp_{\mu}}\right)$. These cuts are listed in Table 6. Type I events required a clean separation of the muon track from the primary vertex and a modest $P_{\perp}$ of the muon. Type III events represented the subset of Type I events in which the muon track was associated with a secondary decay vertex. The reconstructed muonic vertex allowed the cut values on $\mathrm{P}_{\perp \mu}$ and $b_{\mu}$ to be reduced slightly to increase the yield. Possible beauty events, Type II events, were selected on the basis of the larger $P_{\perp}$ spectrum seen in Monte Carlo simulations of beauty decays. Type IV events were selected because of a reconstructed decay vertex outside the emulsion. These Type IV events were used for $K_{g}^{\circ}$ momentum analysis and 


\begin{tabular}{|l|c|c|c|c|}
\hline Cut Variable & Class 3 & Class 2 & Class 1 & Class 4 \\
\hline \hline $\mathbf{P}_{\mu}$ & 8.00 & 8.00 & 8.00 & 0.00 \\
\hline $\begin{array}{l}\mathrm{P}_{\perp \mu} \text { wrt Beam } \\
(\mathrm{GeV} / \mathrm{c})\end{array}$ & 0.20 & 0.80 & 0.250 & 0.00 \\
\hline $\begin{array}{l}\text { Impact Parameter of } \\
\text { Muon (microns) }\end{array}$ & 50 & 100 & 100 & 0 \\
\hline P $_{\text {hadrons (GeV/c) }}$ & 5.0 & 0.0 & 0.0 & 0.0 \\
\hline $\begin{array}{l}\text { Impact Parameter of } \\
\text { Hadrons (microns) }\end{array}$ & 0.050 & 0.0 & 0.0 & 0.0 \\
\hline Decay Length (mm) & 2.0 & 0.0 & 0.0 & 0.0 \\
\hline $\begin{array}{l}\text { Invariant Mass } \\
\left(G e V / c^{2}\right)\end{array}$ & 0.250 & 0.0 & 0.0 & 0.0 \\
\hline
\end{tabular}

Table 6: Listing of the four different classes of cuts used in selecting events for emulsion scamning.

some preliminary charm analysis (done without emulsion information $[28,29,30,31,33]$ ). If an event passed Type I, II, and III cuts, the tracking and vertex information was sent to the emulsion scanners in Japan to scan the event.

Event loss during offline scan selection for a typical vertical emulsion module is listed in Table 7 taken from [30].

\subsection{Emulsion Scanning}

The two different emulsion modules, horizontal and vertical, had two different scanning techuiques. The horizontal scanning was done mainly by human eye whereas the vertical scanning incorporated a sophisticated automated emulsion scanner. The automation of the vertical scanning increased the scanning fate. Since only 3 out of the 37 charm pairs came from the horizontal modules, the scanning technique used for the vertical modules will be 


\begin{tabular}{|l|c|c|}
\hline $\begin{array}{l}\text { Number of } \\
\text { Events } \\
\text { Before Cut(s) }\end{array}$ & Cut(s) description & $\begin{array}{c}\text { Percent } \\
\text { of Events } \\
\text { Cut }\end{array}$ \\
\hline 17450 & $\begin{array}{c}\text { Event is readable from tape and } \\
\text { is within the fiducial volume }\end{array}$ & 30.1 \\
\hline 12093 & Fully reconstructed muon & 71.3 \\
\hline 3469 & No primary vertex reconstructed & 1.0 \\
\hline 3331 & Z of primary upstream of the & \\
& emulsion block & 1.9 \\
\hline 3268 & Z of the primary downstream of the & 12.8 \\
\hline \hline & emulsion block & \# of events \\
\hline 2849 & Class 1 cuts & 116 \\
& Class 2 cuts & 23 \\
& Class 3 cuts & 105 \\
& Class 4 cuts & 113 \\
\hline
\end{tabular}

Table 7: Typical emulsion scanning predictions for a vertical emulsion module.

discussed briefly.

\subsubsection{Automated Scanning}

The last plate of the vertical emulsion stack, the analysis plate, was measured by the automatic scanner. This scanner consists of a video image microscope with a computer driven stage. The movable stage had a position read-out with a $1 \mu \mathrm{m}$ accuracy. The focal length of the microscope is also controlled by the computer and was varied in $10 \mu \mathrm{m}$ intervals. The last emulsion plate has two $80 \mu \mathrm{m}$ thick layers of emulsion, so there were 16 measurements corresponding to 16 different focal lengths. This allowed measurement of the track angle and position at exit of the emulsion. These angles and intercepts were then matched to tracks reconstructed by the VSSD's. Tracks that have matches were then projected onto 


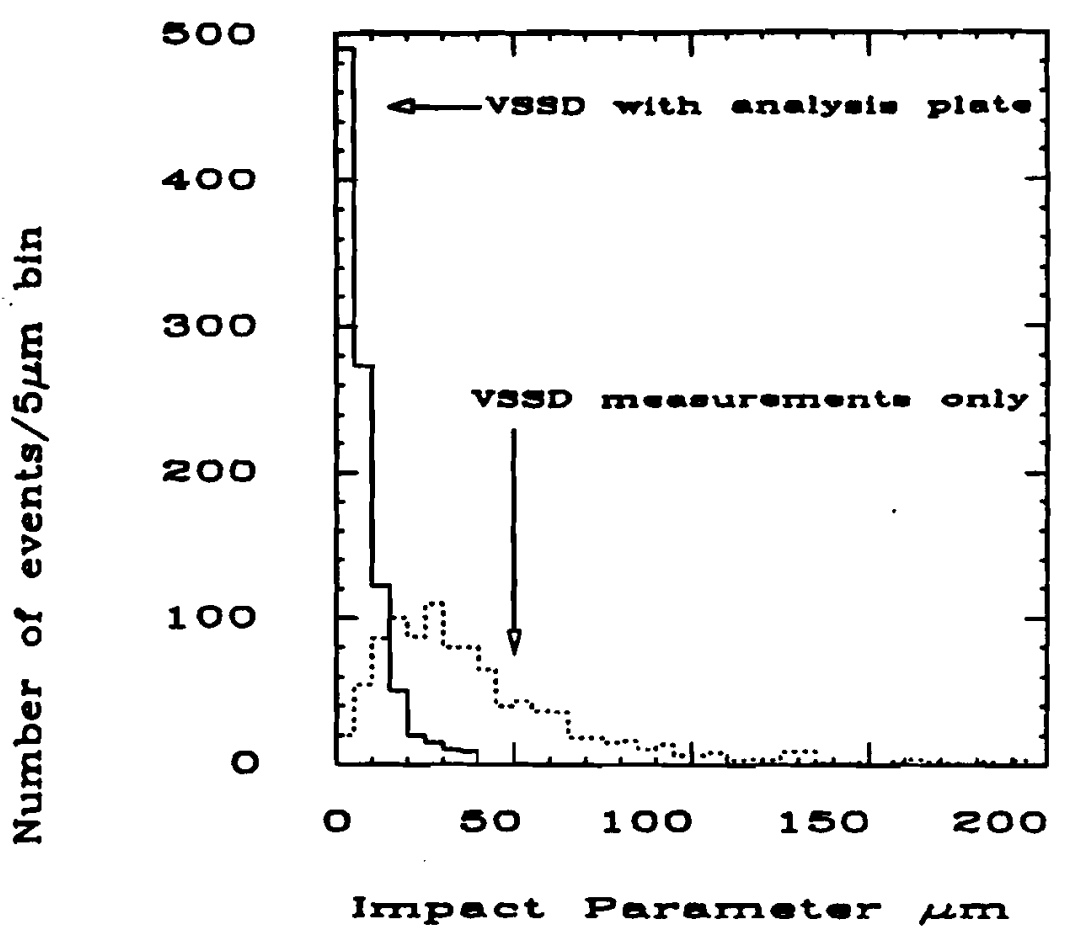

Figure 29: Impact paramter for tracks coming from the primary vertex.

the last emulsion pellicle of the main emulsion block.

The analysis plate information with its $1 \mu \mathrm{m}$ resolution allows a more precise measurement of the impact parameter of the tracks with respect to the primary position. Figure 29 shows the impact parameter for VSSD tracks confirmed to be coming from the primary vertex, the dotted line is for the impact parameter calculated using VSSD information only and the solid line is the recalculated impact parameter using the analysis plate information. Willh this improved impact parameter resolution events with the $\mu$ coming from the primary interaction were eliminated. 


\subsubsection{Graphic Analysis}

Graphic analysis refers to using the primary vertex position and analysis plate track information to quickly eliminate uninteresting tracks from the primary vertex. A computer generated image of the analysis plate track segments and VSSD reconstructed tracks. Tracks were humanly matched between the emulsion and VSSD. Any tracks that were reconstructed by the VSSD's but not attached to the primary vertex were possible decay daughters. Events with a low $P_{t}$ muon with respect to its parent, as measured in the emulsion, direction were rejected at this stage as $K \rightarrow \mu$ decays. If the event passed this graphic analysis the event was saved for complete emulsion scanning.

\subsubsection{Decay Search}

The decay search started by scanning back all tracks that had a match at the analysis plate but were not linked to the primary interaction. This search path ended at the source of the daughter track, either a decay vertex or a secondary interaction vertex. Tracks that originated at the primary but had no match at the analysis plate were scanned starting from the primary vertex until the track led to a decay vertex or the downstream edge of the emulsion. In this search charged decays that had failed reconstruction could be located. Table 8 lists the fraction of events that survived the different stages of emulsion scanning and the time needed to do the measurements. 


\begin{tabular}{|l|c|c|}
\hline $\begin{array}{l}\text { Emulsion } \\
\text { analysis }\end{array}$ & $\begin{array}{c}\text { Percent that } \\
\text { survived (\%) }\end{array}$ & $\begin{array}{c}\text { Length of time } \\
\text { (minutes) }\end{array}$ \\
\hline Fiducial volume cuts & 80 & 0 \\
\hline $\begin{array}{l}\text { Locate Primary } \\
\text { Vertex }\end{array}$ & 95 & 5 \\
\hline $\begin{array}{l}\text { Analysis plate measurements } \\
\text { reject if } \mu \text { coming from primary }\end{array}$ & 33 & $5-10$ \\
\hline $\begin{array}{l}\text { Grapluic analysis } \\
\text { reject low } P_{t} \mu \text { 's }\end{array}$ & 50 & 10 \\
\hline $\begin{array}{l}\text { Decay search } \\
\text { reject if no decay is found }\end{array}$ & 20 & 60 \\
\hline \hline Totals & 3.1 & $80-85$ \\
\hline
\end{tabular}

Table 8: Event through put for the different emulsion scanning stages.

\subsubsection{Interaction Background}

The emulsion technique also rejected secondary interactions in the emulsion block. The presence of heavily ionizing tracks indicative of nuclear breakup were clear indications of non-diffractive secondary interactions. All the events that were considered charm events went through a second pass of scanning. One of the objectives of this second scan was to search for the presence of 'blobs' that would indicate de-excitation of the nucleus after a diffractive interaction. This powerful teclınique provided a sample of charm decays with small backgrounds from interactions in the emulsion block.

\subsection{Emulsion Charm Selection}

Once a decay vertex was located in the emulsion the event had to pass several criteria for it to be saved as a charm candidate event. The muon track reconstructed by the spectrometer must not be linked to the primary vertex. Events with the reconstructed muon linked 
to the primary vertex or linked to a secondary interaction vertex were rejected as charm candidates. The emulsion scanners must find at least one decay vertex in the emulsion. A vertex was a candidate decay vertex if the enulsion scanners did not see any heavily ionizing tracks from nuclear break up coming from the vertex.

If a decay candidate was found the event was completely measured by the enulsion scanners. The emulsion measurements started by matching spectrometer tracks to the primary vertex tracks. Unmatched primary tracks (emulsion tracks without a spectrometer match) were then scanned to the end of the emulsion block or until the track ended in a secondary vertex. Unmatched spectrometer tracks (spectrometer tracks without an emulsion match) were located at the downstream end of the emulsion block and scanned back towards the primary vertex until the source (neutral decay vertex) of the track was located. This process of following tracks down from the primary and scaming back towards the primary provided a way to pick up both charged and neutral decays. Upon completion of emulsion scanning, the topology of the enulsion decay vertices was known.

\subsubsection{Decay Topologies}

The decays were classified by the following topologies. First the decays were broken into two groups depending on whether the muon track was attached to the decay. Decays with attached $\mu$ 's were called muonic decays since they involved the trigger $\mu$. Decays without attached $\mu$ 's were called unbiased or hadronic decays. The hadronic name was somewhat erroneous since this decay sample also had electron daughters or low momentum muon daughters about $20 \%$ of the time. In addition to the muon tag, the events were tagged by 
the number of charged tracks or prong count and charge of the parent. Charged decays into one charged track were called C-1's and three charged tracks were called C-3's, five tracks were called C-5's. Neutral decays into two prongs were called V-2's and four prongs V-4's.

The notation that evolved in the E653 classification was to place a $\mu$ after the prong count if the decay had a muon daughter - a neutral V-2 decay with a tagged muon was written $\mathrm{V}-2 \mu$. If there was no $\mu$ assignment, the decay was assumed to be hadronic - V-2 implies a hadronic V-2 decay.

At this point, the event was selected as a charm event by topology alone with some cuts on the muon track. The decays found in the emulsion were tagged visually and nondiffractive secondary interactions were eliminated by the presence of heavily ionizing tracks. The next stage in the analysis was to use the emulsion information to help the spectrometer tracking and to reconstruct the decays outside the emulsion block.

\subsection{Refitting}

If at least one decay was found in the emulsion, complete measurements were made by the emulsion scanners of all vertex positions in the emulsion and emulsion track slopes for tracks that did not have a spectrometer match. The emulsion scanners did not measure the track slope if the emulsion track had a spectrometer match since the spectrometer track slope resolution was 3 orders of magnitude better than the emulsion (See Table 5). Emulsion measurements were then used to guide the spertrometer track reconstruction. Specifically, emulsion track information was used to predict the track path through the VSSD's allowing 
the VSSD's to use a more relaxed criterion to reconstruct tracks. Once all the emulsion tracks had been matched to spectrometer tracks, the reconstruction program then used the left over hits in the VSSD's to reconstruct tracks not matched to the emulsion tracks.

The unmatched spectrometer tracks were then used to form decay vertices outside the emulsion block. These vertices were called counter or computer vertices and formed another distinct topology of decays. Once a counter vertex was reconstructed, the ummatched emulsion tracks were checked to see if they pointed to the counter vertex position. A counter vertex was considered a good charm decay candidate if the sum of the charge tracks was 0 or \pm 1 (there were some \pm 2 or \pm 3 vertices) and if it had (had not) an emulsion parent it was a charged (neutral) decay. The complete event topology was established at this point and the next step in charm selection was to use the momentum information of the linked tracks to determine if the decay was consistent with a charm decay. The emulsion scanners found 1205 events that contained a decay with a topology consistent with charm decay.

\subsection{Final Charm Pair Sample}

A progression of cuts was applied to the 1205 charm candidates found by the emulsion scanners to obtain a sample of charm pairs. These cuts are listed in Table 11 and are described in the following sections. Without particle identification, a set of cuts was developed that utilized the topological properties of charm pair events. The kinematic quantities of the decays were also used in the final cut to ensure that the dec?' $\checkmark$ were consistent with charm decays. 


\subsubsection{Level 1: A Muonic Vertex}

The trigger muon was used not only to trigger the data acquisition system but was also used offline to select events for emulsion scanning. The understanding of the efficiencies for the analysis required that one of the charm decays had the trigger muon as a daughter. This requirement removed the serendipitous charm found by the emulsion scanners. These serendipitous events were initially kept by the emulsion scanners since there was a decay (non-muonic) found in the emulsion and it was hoped that the refitting procedure would reconstruct a partner muonic decay outside the emulsion. There were 344 events that failed this cut.

\subsubsection{Level 2: Two or more decay vertices}

A complete charn event must have two charm decays reconstructed. The level 2 cut checked the vertex list and saved only those events that had two or more decay vertices. At least one of the decays must reside in the main emulsion block, this allowed the analysis to use the visual power of the enulsion to check the decay vertex for nuclear break up indicative of a secondary interaction. A good "counter" vertex was required to have 2 or more charged daughters and to be located outside the main emulsion block. The counter vertex was also required to be a muonic vertex; the number of counter hadronic vertices reconstructed with a charge sum of \pm 2 for V-2's and V-4's or \pm 3 for C-3's demonstrated that the hadronic counter vertices were consistent with being all background (See section 5.4 for details). One of the decays must also be the muonic decay required in the previous cut level. There were 409 events that had at least two vertices that passed these cuts. 


\subsubsection{Level 3: Complete tracking}

The decay daughters must be fully linked through the spectrometer to determine the momentum for the charged daughters. This tracking requirement allowed more sophisticated charm selection than just topological identification and allowed a momentum estimate (see Chapter 6) for the charm parent to be obtained. In addition to requiring complete tracking of the decay daughters, the cuts of the previous levels were still enforced, the event now consisted of at least two decay vertices which were fully reconstructed. At least one of the decays must be in the emulsion, and at least one of the decays must be a muonic decay. The number of fully reconstructed events with at least two vertices was 273 .

\subsubsection{Level 4: $M_{\min }$ Cuts}

Without extensive particle identification the task of determining if a decay was consistent with a charm meson decay was not easy, but by relying on the strengths of the spectrometer (excellent vertex positions, momentum resolution, and muon identification) a parameter called $M_{\min }$ was developed which enabled charm event selection and background determination [24].

\section{WHAT IS $\mathrm{M}_{\min }$ ?}

The high resolution of the vertex positions $(2 \mu \mathrm{m})$ enabled the transverse momentum imbalance with respect to the charm parent to be well measured. This imbalance was called the missing $P_{t}$ of the vertex and was the transverse momentum carried by the neutral daughters. The minimum mass associated with the decay was calculated by 
assuming that the charged and neutral systems, in the rest frame of the $D$, were produced transverse to the boost direction and were assigned particles masses using the mass assignments described in Section 6.2.1. $M_{v i}$ is the effective mass for the charged tracks and $M_{\text {reut }}$ is the mass assignment for the neutral particle. The value $M_{\min }$ in equation 26 is identical to the value obtained by solving for the mass that yields only one solution from the $0-\mathrm{C}$ equation for the decay hypothesis and for this historical reason it is call $M_{\min }$.

$$
M_{\min }=\left(M_{v i s}^{2}+P_{t}^{2}\right)^{\frac{1}{2}}+\left(M_{\text {neut }}^{2}+P_{t}^{2}\right)^{\frac{1}{2}}
$$

The distributions for $M_{\min }$ depended on the topology in question and how many missing neutrals were involved. Single neutral decays had a $M_{\min }$ distribution that peaked at the charm mass whereas multiple neutral modes had a rounded distribution that peaked well below the nominal charm mass. For this analysis the decay branching ratios in Table 23 were used. Figure 30 shows the $\mathrm{M}_{\min }$ distribution for $\mathrm{C}-1 \mu \mathrm{V}-2 \mu$ and $\mathrm{C}-3 \mu$ charm decays, the solid curve shows the Monte Carlo distribution, and the dashed curve is for the data before any cuts were applied. Figure 31 shows the $M_{\min }$ distribution from the hadronic modes, C-1, V-2 and C-3. The $M_{\min }$ for each decay was required to be within topologically dependent bounds described below, the error $(\sigma)$ on the measured value of $M_{\min }$ was used to allow all properly reconstructed charm decays to be accepted by these $M_{\min }$ cuts. The M.C. $M_{\min }$ curves are compared to the data $M_{\min }$ distributions in the following sections. 
MUONIC C-1

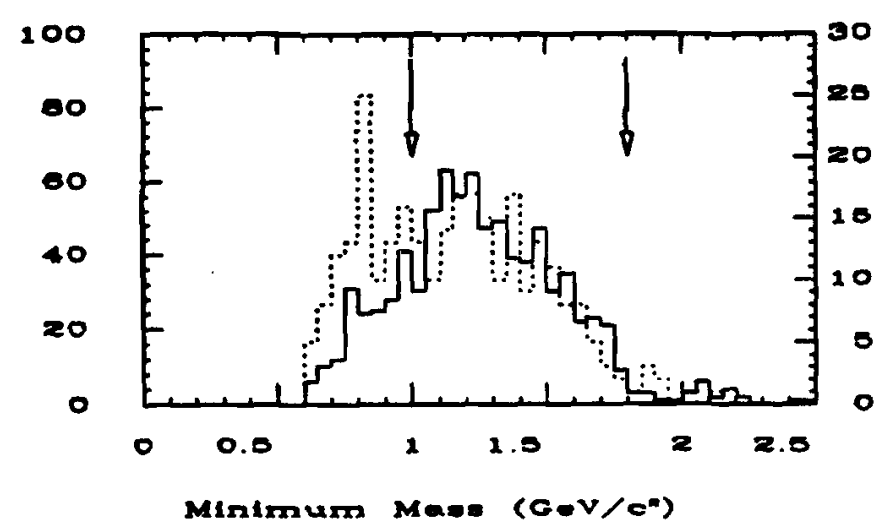

MUONIC $v-2$

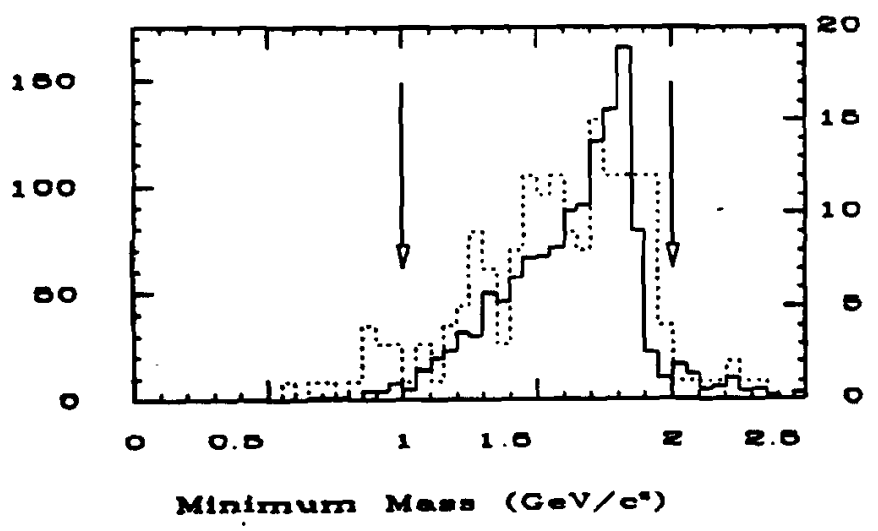

MUONIC C-3

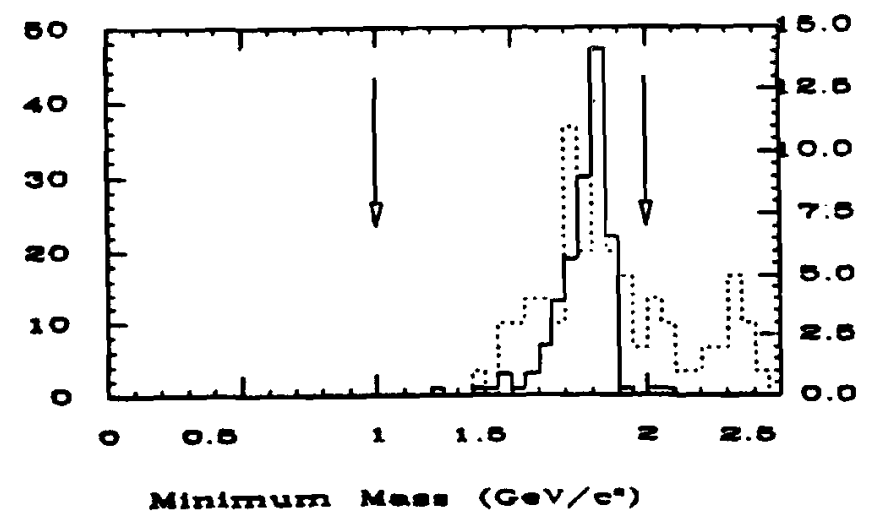

Figure 30: $M_{\min }$ distributions for muonic decays of $D^{\circ}$ and $D^{+}$mesons. The solid curve is for Monte Carlo and the dashed represents the data before any cuts. The arrows point to the upper and lower bounds on $M_{\min }$ required for a decay to be considered charm. 
MADRONIC C-1

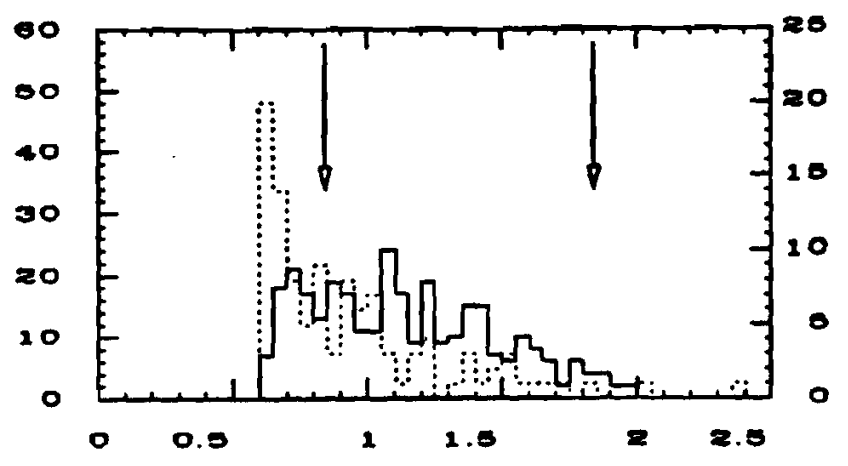

Minimum Maga (cov/on)

HADRONIC $v-\varepsilon$

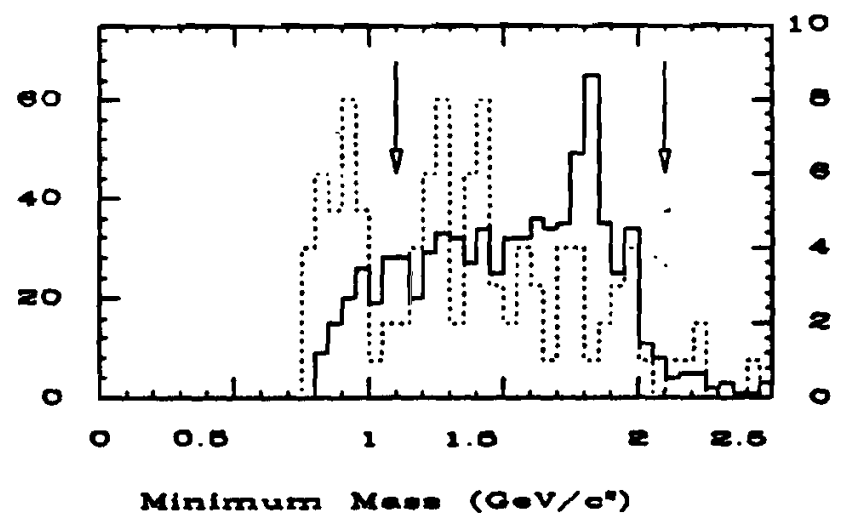

HADRONIC C-3

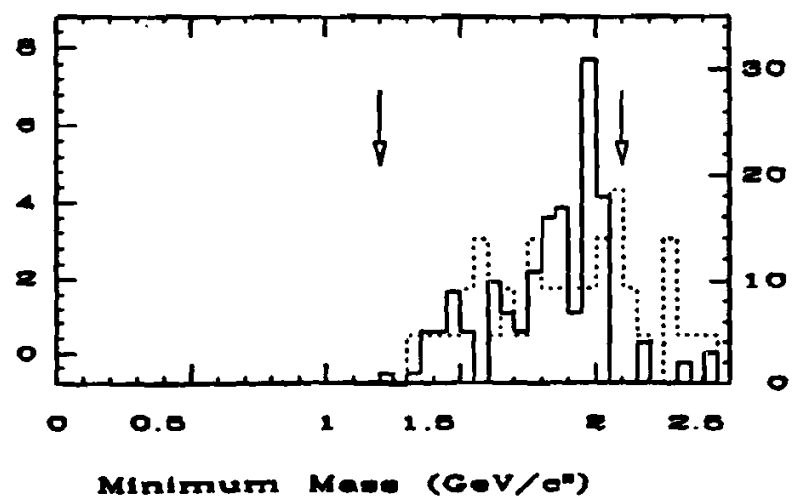

Figure 31: $M_{\min }$ distributions for non-muonic decays of $D^{\circ}$ and $D^{+}$mesons. The solid curve is for Monte Carlo and the dashed represents the data before any cuts. The arrows point to the upper and lower bounds on $M_{\text {mir }}$ required for a decay to be considered charm. 


\section{Muonic Kink Decays (C-1 $\mu$ 's)}

The $M_{\min }$ for the muonic kink decays in the complete data sample agreed well with the Monte Carlo $M_{\min }$ at high $M_{\min }$. The discrepancy at low $M_{\min }$ was due to $K^{- \pm} \rightarrow \mu \nu$ decay. This strange background was eliminated by placing the low $M_{\min }$ cut at $(1.0-1 \sigma)$ $\mathrm{GeV} / \mathrm{c}^{2}$ and by requiring the $\mathrm{P}_{t}$ of the $\mu$ with respect to the parent direction to be greater $\operatorname{than} 0.250 \mathrm{GeV} / \mathrm{c}$.

\section{Muonic V-2 Decays}

The raw $M_{\min }$ distribution for the V-2 $\mu$ 's agreed very well with the Monte Carlo. The small bump at low $M_{\min }\left(\right.$ at $0.9 \mathrm{GeV} / \mathrm{c}^{2}$ ) was due to $K_{s} \rightarrow \pi \pi$ decays where one of the $\pi$ 's then decayed into a $\mu$ which triggered the apparatus. These strange decays were eliminated by placing the lower $M_{\min }$ cut at $(1.0-1 \sigma) \mathrm{GeV} / \mathrm{c}^{2}$ and requiring one of the decay daughters to have a $P_{t}$ with respect to the parent direction of greater then 0.250 $\mathrm{GeV} / \mathrm{c}^{2}$ and by also explicitly cutting on the $\pi \pi$ mass of the vertex (See Table 9 ).

\section{Muonic C-3 Decays}

The high $M_{\min }$ tail in the C-3 $\mu M_{\min }$ data distribution was due to two possible sources:

- Charm background: Hadronic feed through where one of the $K$ 's or $\pi$ 's from a $D^{ \pm}$ decay subsequently decayed into a $\mu$ which triggered the apparatus and was reconstructed by the spectrometer. 
- Non-charm background: Diffractive interactions which can not be flagged as secondary interactions by the emulsion scanners.

These two sources of background are explained in more detail in the following chapter on background determination in the charm sample. Muonic tridents with high $M_{\text {min }}$ can be attributed to mis-identified hadronic charm decays and were included in the sample by placing the upper $M_{\min }$ at $(2.0+2 \sigma) \mathrm{GeV} / \mathrm{c}^{2}$.

\section{Hadronic C-1's}

The $M_{\min }$ distribution for the hadronic C-1's in the complete data sample showed a strong peaking at low $M_{\min }$. The peak at low $M_{\min }$ was due to small angle scattering in the emulsion. The low $M_{\min }$ kinks were eliminated by placing the lower cut on $M_{\min }$ at $(0.80-1 \sigma) \mathrm{GeV} / \mathrm{c}^{2}$, and a $P_{t}>0.250 \mathrm{GeV} / \mathrm{c}$ cut on the missing $P_{t}$ of the vertex.

\section{Hadronic V-2's}

The hadronic V-2's were contaminated predominantly by three sources of background: $\mathrm{K}_{s}^{0}$ and $\Lambda^{\circ}$ decays and $\gamma$ conversions. These sources of background appeared at the low end of $M_{\min }$ distribution and were eliminated from the charm pair sample by placing the $M_{\min }$ cut at $(1.1-\sigma) \mathrm{GeV} / \mathrm{c}^{2}$ and by explicitly cutting on $M_{\pi \pi}$ of the decay (see Table 9 ).

\section{Hadronic C-3 Decays}

The $M_{\min }$ distribution for the hadronic C-3's had the interesting attribute of peaking at $2.0 \mathrm{GeV} / \mathrm{c}^{2}$ instead of at the nominal charm mass $\left(1.867 \mathrm{GeV} / \mathrm{c}^{2}\right)$. This over shoot of the 


\begin{tabular}{|ccc|}
\hline $\begin{array}{c}\text { Lower Bound } \\
\mathrm{GeV} / \mathrm{c}^{2}\end{array}$ & $\begin{array}{c}\text { Upper Bound } \\
\mathrm{GeV} / \mathrm{c}^{2}\end{array}$ \\
\hline 0.310 & $<M_{\pi \pi}<$ & 0.490 \\
\hline 0.504 & $<M_{\pi \pi}$ \\
\hline
\end{tabular}

Table 9: Allowed range for $M_{\pi \pi}$ of neutral V-2 decays.

charm mass was due to the all charged mode of $D^{+} \rightarrow K^{-} \pi^{+} \pi^{+}$. For this reason the upper $M_{\min }$ cut was placed at $(2.1+2 \sigma) \mathrm{GeV} / \mathrm{c}^{2}$ to include as many of the high $M_{\min }$ decays as possible.

\section{Hadronic V-4 Decays}

There were no cuts on the $M_{\min }$ for the neutral V-4 decays found in the emulsion. The hadronic V-4's represented a very clean sample since there is no source for strange contamination and the neutral diffractive background was very low.

Table 10 liststhe allowed ranges for $M_{\min }$ by topology - if a decay had an acceptable $M_{\min }$ it was considered a charm candidate.

\subsubsection{Level 5: Multiprong-Multiprong Charm pairs}

The momentum estimator, to be described in Chapter 6, worked for all topologies but the resolution of the estimate became poor when most of the parent momentum was carried away by neutral daughters. The study of the kinematic distributions for the charm pair sample required the momentum of the two charm parents to be known with some 


\begin{tabular}{|l|c|c|}
\hline Topology & $\begin{array}{c}\text { Lower Bound } \\
\text { on } M_{\min }\left(\mathrm{GeV} / \mathrm{c}^{2}\right)\end{array}$ & $\begin{array}{c}\text { Upper Bound } \\
\text { on } M_{\min }\left(\mathrm{GeV} / \mathrm{c}^{2}\right)\end{array}$ \\
\hline \hline C-1 Hadronic & $0.80-1 \sigma$ & $1.84+2 \sigma$ \\
\hline C-1 Muonic & $1.0-1 \sigma$ & $1.80+2 \sigma$ \\
\hline V-2 Hadronic & $1.1-1 \sigma$ & $1.95+2 \sigma$ \\
\hline V-2 Muonic & $1.0-1 \sigma$ & $1.87+2 \sigma$ \\
\hline C-3 Hadronic & $1.2-1 \sigma$ & $2.10+2 \sigma$ \\
\hline C-3 Muonic & $1.0-1 \sigma$ & $2.00+2 \sigma$ \\
\hline V-4 Hadronic & No cuts & No cuts \\
\hline
\end{tabular}

Table 10: $M_{\min }$ cut values for the different topologies. The sigma is the measured error on the $M_{\min }$ for the decay.

\begin{tabular}{|c|l|c|}
\hline Cut Level & Type of Cut & Number of Events that passed \\
\hline \hline-1 & $\begin{array}{l}\text { Type 1,2,3 events selected } \\
\text { for emulsion scanning }\end{array}$ & 56283 \\
\hline 0 & $\begin{array}{l}\text { Events with charm decay candidates } \\
\text { found by emulsion scanners }\end{array}$ & 1205 \\
\hline 1 & $\begin{array}{l}\mu \text { track attached to a } \\
\text { decay vertex }\end{array}$ & 861 \\
\hline 2 & $\begin{array}{l}\text { At least two decay vertices / } \\
\text { at least one in the emulsion } \\
\text { at least one muonic } \\
\text { uo counter C-1's, C-1 } \mu, \text { V-2's, C-3's }\end{array}$ & 409 \\
\hline 3 & $\begin{array}{l}\text { At least two decay vertices with } \\
\text { complete tracking / at least } \\
\text { one muonic / at least one in } \\
\text { the emulsion }\end{array}$ & $\begin{array}{l}\text { M Cuts } \\
\text { C-1's,C-1 } \mu \text { 's } \\
\text { allowed in sample }\end{array}$ \\
\hline 4 & $\begin{array}{l}\text { Fully reconstructed multiprong-multiprong } \\
\text { charm pairs }\end{array}$ & 953 \\
\hline
\end{tabular}

Table 11: Succession of cuts applied to the charm candidate sample. 


\begin{tabular}{|l|c|c|c|}
\hline $\begin{array}{l}\text { Hadronic } \rightarrow \\
\text { Muonic } \downarrow\end{array}$ & V-2 & C-3 & V-4 \\
\hline V-2 $\mu$ & 12 & 3 & 4 \\
\hline C-3 $\mu$ & 3 & 2 & 1 \\
\hline
\end{tabular}

Table 12: Emulsion-emulsion charm pair topologies in the final charm pair sample.

‘

\begin{tabular}{|l|c|c|c|c|c|}
\hline $\begin{array}{l}\text { Enulsion } \rightarrow \\
\text { Counter } \downarrow\end{array}$ & V-2 & V-2 & C-3 & C-3 $\mu$ & V-4 \\
\hline V-2 $\mu$ & 1 & 1 & 5 & & 3 \\
\hline $\mathrm{C}-3 \mu$ & 1 & & 1 & & \\
\hline
\end{tabular}

Table 13: Emulsion-counter charm pair topologies in the final charm pair sample.

accuracy for the momentum of the charm pair to be determined. For this reason the final charm pair sample used for kinematic study involved only those events with both charm mesons decaying into two or more charged particles. This final cut leaves 37 multiprong-multiprong charm pair events. Table 12 lists the topology of the 25 emulsion-enulsion events in the final 37 charm pairs and Table 13 lists the topology for the 12 emulsion-counter charm pairs. 


\section{Chapter 5}

\section{Backgrounds}

\subsection{Introduction}

The charm pair event selection described in the previous chapter eliminated vertices if they were not fully reconstructed or if their $M_{\min }$ was inconsistent with charm decay. The cuts did not eliminate reconstructed background that had an acceptable value of $M_{\text {min }}$. This chapter will study how many of the decays and events in the charm pair sample were attributed to non-charm vertices and reconstruction errors. A source of non-charm vertices was diffractive interactions in the emulsion that can not be tagged by the presence of heavily ionizing tracks in the emulsion. Reconstruction errors were from two sources, track matching between the emulsion and spectrometer, and errors in the counter vertex reconstruction. The background from these two sources will be calculated in this chapter and shown to be small. 


\begin{tabular}{|l|c|c|}
\hline Topology & $\begin{array}{c}\text { Found } \\
\text { Non-Diffractive }\end{array}$ & $\begin{array}{c}\text { Scaled } \\
\text { Diffractive }\end{array}$ \\
\hline \hline $\mathrm{C}-1$ & 18 & $5.0 \pm 1.2$ \\
\hline $\mathrm{C}-1 \mu$ & 16 & $4.5 \pm 1.1$ \\
\hline $\mathrm{V}-2$ & 6 & $1.68 \pm 0.69$ \\
\hline $\mathrm{V}-2 \mu$ & 5 & $1.40 \pm 0.63$ \\
\hline $\mathrm{C}-3$ & 2 & $0.56 \pm 0.39$ \\
\hline $\mathrm{C}-3 \mu$ & 6 & $1.68 \pm 0.69$ \\
\hline V-4 & 4 & $1.12 \pm 0.56$ \\
\hline TOTAL & 57 & $15.9 \pm 2.11$ \\
\hline
\end{tabular}

Table 14: The number of non-diffractive vertices with all light shower tracks match to the spectrometer, scaled to yield an estimate of the diffractive background in the complete data set.

\subsection{Diffractive Background}

The amount of diffractive interaction background was estimated by requesting from the emulsion scanners the number of non-diffractive neutral interactions in which light shower tracks were produced in addition to the heavily ionizing nuclear break up tracks indicative of non-diffractive interactions. Non-diffractive interactions that have the light shower tracks matched to the spectrometer were taken to reflect the number of diffractive interactions matched. The non-diffractive interactions was scaled by the ratio of diffractive to non-diffractive interactions seen in the emulsion (using white stars), i.e. 10\%. Table 14 list the number of non-diffractive interactions found in 10,711 emulsion scan events by the topology reflected by the light shower tracks. The found non-diffractive events must be scaled up by 2.8 since the information listed does not include the complete date set. The scaled diffractive numbers represent the total number of diffractive events in the entire data set. The final amount of diffractive background was calculated assuming a 


\begin{tabular}{|l|c|c|c|c|c|c|}
\hline Topology & $\begin{array}{c}\text { Total } \\
\text { Number } \\
\text { of Decays }\end{array}$ & $\begin{array}{c}\text { Total } \\
\text { Diffractive } \\
\text { Background }\end{array}$ & $\begin{array}{c}\text { Decays } \\
\text { at } \\
\text { Level 4 }\end{array}$ & $\begin{array}{c}\text { Diffractive } \\
\text { Background } \\
\text { at Level 4 }\end{array}$ & $\begin{array}{c}\text { Decays } \\
\text { at } \\
\text { Level 5 }\end{array}$ & $\begin{array}{c}\text { Diffractive } \\
\text { Background } \\
\text { at Level 5 }\end{array}$ \\
\hline C-1 & 140 & $5.04 \pm 1.19$ & 30 & $1.08 \pm 0.26$ & X & X \\
\hline C-1 $\mu$ & 273 & $4.48 \pm 1.12$ & 33 & $0.54 \pm 0.14$ & X & X \\
\hline V-2 & 92 & $1.68 \pm 0.69$ & 29 & $0.53 \pm 0.22$ & 17 & $0.31 \pm 0.13$ \\
\hline V-2 $\mu$ & 179 & $1.40 \pm 0.63$ & 36 & $0.28 \pm 0.13$ & 20 & $0.16 \pm 0.07$ \\
\hline C-3 & 44 & $0.56 \pm 0.39$ & 17 & $0.22 \pm 0.15$ & 11 & $0.14 \pm 0.10$ \\
\hline C-3 $\mu$ & 100 & $1.68 \pm 0.69$ & 9 & $0.15 \pm 0.06$ & 6 & $0.10 \pm 0.04$ \\
\hline V-4 & 23 & $1.12 \pm 0.56$ & 13 & $0.63 \pm 0.32$ & 7 & $0.43 \pm 0.22$ \\
\hline TOTAL & 851 & $15.9 \pm 2.11$ & 167 & $3.43 \pm 0.53$ & 61 & $1.14 \pm 0.29$ \\
\hline
\end{tabular}

Table 15: Diffractive background estimates in the complete data set and the charm pair data set at the last two stages of charm pair selection.

constant percentage of diffractive background. This assumption leads to a very conservative estimate for the diffractive background.

The emulsion scanners have checked each decay vertex in the decay sample for associated 'blobs' at the decays. These 'blobs' indicate de-excitation of a nucleus after a diffractive interaction. Decays with 'blobs' attached to the decay vertex were removed from the charm candidate list. However the efficiency for finding these diffractive interactions was not determined and we are forced to use the best esitmate quoted in the Table 15. Assuming a constant percentage of background throughout the charm selection process implies that the charm selection process does nothing to enhance the charm signal.

Discussion of sign correlations in the next chapter will show that this is not true. Table 15 lists the number of decays for each emulsion topology for the entire data set and for the events in the charm pair sample at level 4 and 5 of the selection process.

The diffractive background in the final data set is on the order of one ev rnt. The study of the charge correlations in the charm pair events is another way to study the diffractive 
background in the charged decays.

\subsection{Wrong Sign Charged Background}

The reconstructed muon track tagged the charm species produced in the event.

Comparing the sign of the charged decays with the charm species deternnined by the $\mu$ sign in the event allowed the background from the non-charm vertices to be estimated. Figure 32 shows pictorially the definitions described below. Although decays with only one charged daughter (kinks) were cut from the final data set, they provided some insight as to the performance of the charm cuts in reducing background and are described in detail. There are three possible sources for these wrong sign decays.

- The track matching or vertex reconstruction problems.

- Hadronic charm decay where the $\mathrm{K}$ subsequently decays via, $\mathrm{K} \rightarrow \mu \nu$, and is reconstructed by the spectrometer.

- Non-charm vertices, diffractive background.

\subsubsection{Hadronic Kinks (C-1's)}

A hadronic kink (C-1) was consistent with charm decay if it had the opposite charge to the muon in the event. The hadronic kinks were divided into right sign/wrong sign, emulsion vertex/counter vertex and kinks with a $P_{t}<0.250 \mathrm{GeV} / \mathrm{c}$ or $P_{t}>0.250 \mathrm{Ge}: / \mathrm{c}$. Evidence that low $P_{t}$ kinks were inconsistent with a charm hypothesis was seen by 


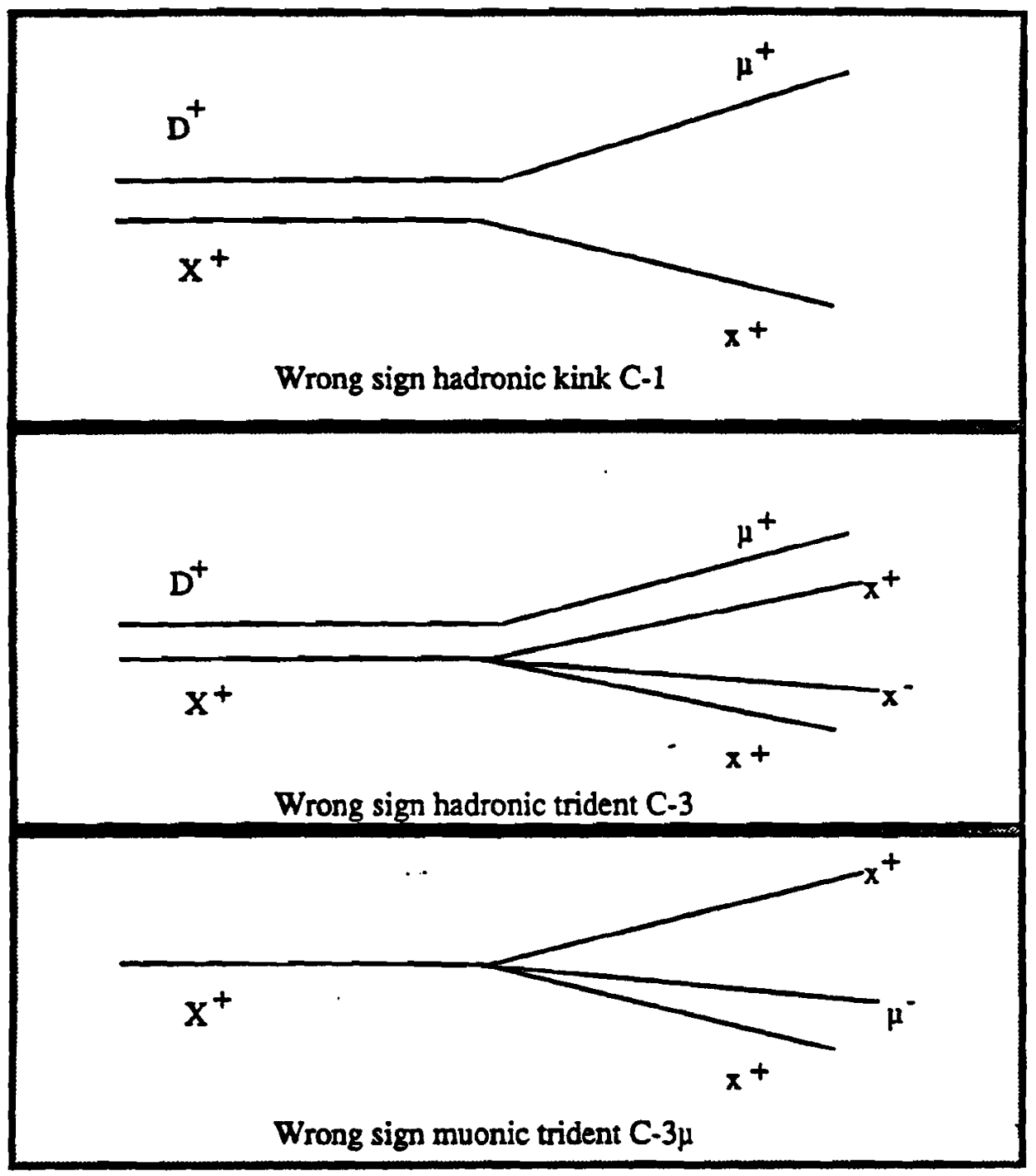

Figure 32: Wrong sign $\mu$ events used to estimate charge background. 


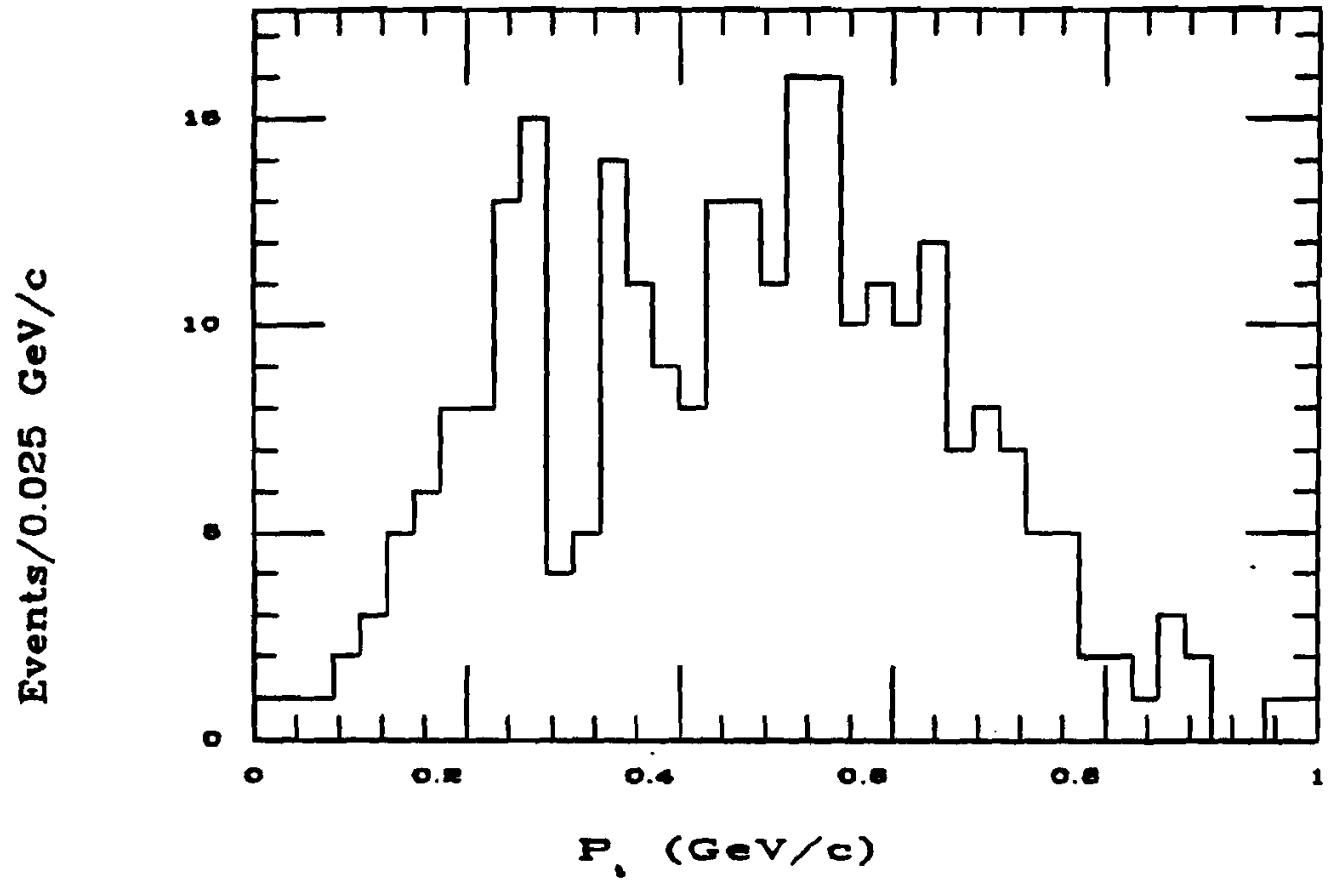

Figure 33: $P_{t}$ distribution for right sign C-1's found in the emulsion.

comparing the $P_{t}$ spectrum for the right sign and wrong sign hadronic C-1's found in the emulsion (Figure 33 and 34). The wrong sign spectrum had a much lower $P_{t}$ distribution than the right sign kinks indicative of small angle scattering in the emulsion. The difference between the two $P_{t}$ distributions also gave one confidence that the $\mu$ in the event was associated with charm decay. Any kinks, hadronic or muonic, had to have a $P_{t}>0.250 \mathrm{GeV} / \mathrm{c}$ for it to be counted as a charm candidate.

The background in the right sign kinks was taken to be the number of wrong sign kinks for the type of kink in question. Table 16 contains the results from the complete data set. From Table 16 we see that all the counter hadronic kinks, regardless of $P_{t}$, were consistent with being all background. All counter kinks were rejected as charm candidates. Emulsion kinks with a $P t>0.250 \mathrm{GeV} / \mathrm{c}$ with the right sign have about $24 \%$ background. The number of wrong sign emulsion kinks at Level 4 is 3 events out of 33 decays, thus the 


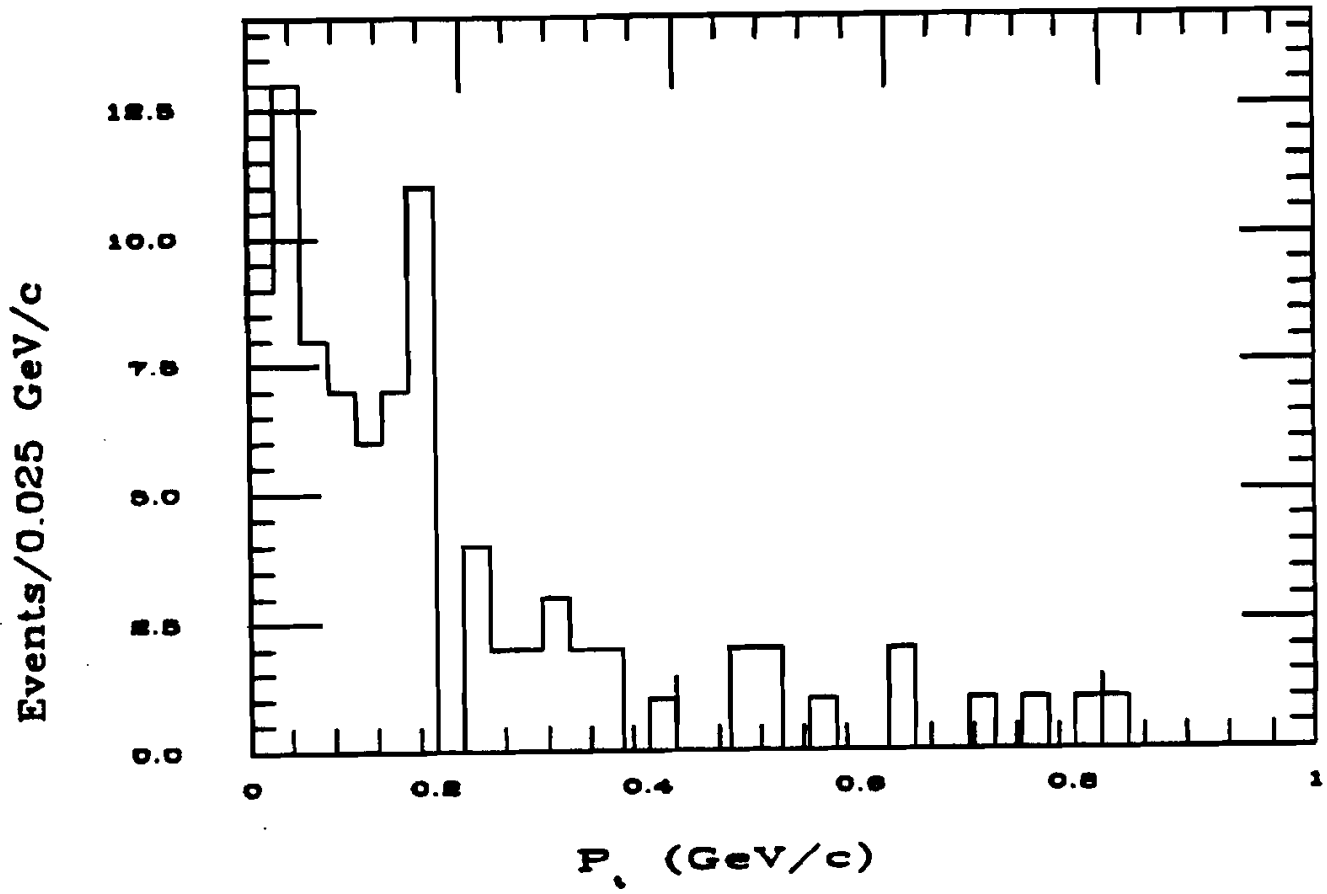

Figure 34: $P_{t}$ distribution for wrong sign C-1's found in the emulsion.

background in the hadronic kinks was reduced by more than a factor of two in the charm selection process.

\subsubsection{Hadronic Tridents (C-3's)}

The sign of a hadronic trident was also required to be opposite the sign of the $\mu$ for the event to be consistent with a charm pair event. Table 17 shows the right sign/wrong sign break down for the hadronic tridents for the entire charm sample. Hadronic C-3's found by the emulsion scanners were consistent with charm decay. The hadronic counter tridents show the same level of background as the hadronic kinks. It is for this reason that counter hadronic tridents were eliminated from the charm sample. The final charm sample contained 11 right sign hadronic tridents located in the emulsion with no wrong sign tridents passing the selection cuts. 


\begin{tabular}{|l|c|c|c|}
\hline Kink & Right Sign & Wrong Sign & Background \\
\hline $\begin{array}{l}\text { Emulsion kinks } \\
\text { with } P t<0.250 \mathrm{GeV}\end{array}$ & 63 & 67 & $106 \%$ \\
\hline $\begin{array}{l}\text { Enulsion kinks } \\
\text { with } P t>0.250 \mathrm{GeV}\end{array}$ & 46 & 11 & $24 \%$ \\
\hline $\begin{array}{l}\text { Counter kinks } \\
\text { with } P t<0.250 \mathrm{GeV}\end{array}$ & 10 & 10 & $100 \%$ \\
\hline $\begin{array}{l}\text { Counter kinks } \\
\text { with } P t>0.250 \mathrm{GeV}\end{array}$ & 6 & 4 & $67 \%$ \\
\hline
\end{tabular}

Table 16: Sign correlations in hadronic kinks with respect to the $\mu$ in the event. Right sign kinks had the opposite sign of the muon sign in the event.

\begin{tabular}{|l|c|c|c|c|}
\hline Vertex Type & Total & Right Sign & Wrong Sign & Background \\
\hline C-3 enulsion & 36 & 33 & 3 & $11 \%$ \\
\hline C-3 counter & 5 & 3 & 2 & $66 \%$ \\
\hline
\end{tabular}

Table 17: Sigu correlation for hadronic tridents (C-3's) with respect to the $\mu$ in the event. Right sign tridents have the opposite charge sum of the muon charge in the event.

\subsubsection{Muonic Tridents (C-3 $\mu$ 's)}

A trident with a muon daughter (C-3 $\mu$ ) had the same sign as the daughter muon if it was from charm decay. Table 18 is a listing of the number of wrong sign/right sign C-3 $\mu$ 's in the complete data sample. The emulsion decays had a higher background than the counter decays due to the amount of charged secondary interactions that occurred in the emulsion. The final charm pair sample did not contain any wrong sign muonic tridents. The Level 4 charm pair sample had one emulsion $\mathrm{C}-3 \mu$.

All the charged topologies ( $\mathrm{C}-1, \mathrm{C}-3$ and $\mathrm{C}-3 \mu$ ) showed a reduction in the number of wrong sign vertices to right sign vertices as the charm selection cuts became more stringent. 


\begin{tabular}{|l|c|c|c|c|}
\hline Vertex Type & Total & Riglit Sign & Wrong Sign & Background \\
\hline C-3 $\mu$ enrulsion & 86 & 65 & 21 & $32 \%$ \\
\hline C-3 $\mu$ counter & 12 & 12 & 0 & $0 \%$ \\
\hline
\end{tabular}

Table 18: Sign correlations for muonic tridents. Right sign means the sign of the muon equals the charge sum of the vertex.

\begin{tabular}{|l|c|c|c|c|}
\hline Topology & $Q= \pm 0$ & $Q= \pm 1$ & $Q= \pm 2$ & $Q= \pm 3$ \\
\hline V-2 emulsion & 76 & & 6 & \\
\hline V-2 $\mu$ emulsion & 157 & & 7 & \\
\hline C-3 emulsion & & 33 & & 2 \\
\hline C-3 $\mu$ emulsion & & 65 & & 3 \\
\hline V-4 emulsion & 19 & & 2 & \\
\hline V-2 counter & 36 & & 31 & \\
\hline V-2 $\mu$ counter & 41 & & 16 & \\
\hline C-3 counter & & 3 & & 3 \\
\hline C-3 $\mu$ counter & & 12 & & 2 \\
\hline
\end{tabular}

Table 19: Wrong charge vertices in the complete data set.

\subsection{Wrong charge vertices}

The track mismatch errors and vertex reconstruction error exhibit themselves in vertices that have a charge sum greater than one. The number of $Q= \pm 2 \mathrm{~V}-2$ 's, $Q= \pm 3 \mathrm{C}-3$ 's and $Q= \pm 2$ V-4's reflects the number of background vertices in the charm sample that had an acceptable charge sum. Table 19 lists the number of wrong charge vertices for the different topologies in the complete data set. The hadronic counter vertices show an unacceptable amount of background and were eliminated from the charm pair analysis at cut Level 2. The Level 4 charm pair sample had 9 wrong charge vertices and 124 vertices with a $Q=0$ or \pm 1 . The $Q= \pm 1$ vertices had to have a sign consistent with the muon sign in the event. This $8 \%$ background was reduced to one decay out of 74 decays in the funal sample. 


\begin{tabular}{|l|c|c|c|c|}
\hline Topology & $\mathrm{Q}= \pm 0$ & $\mathrm{Q}= \pm 1$ & $\mathrm{Q}= \pm 2$ & $\mathrm{Q}= \pm 3$ \\
\hline $\mathrm{V}-2$ emulsion & 28 & & 1 & \\
\hline $\mathrm{V}-2 \mu$ emulsion & 37 & & 2 & \\
\hline $\mathrm{C}-3$ emulsion & & 17 & & 0 \\
\hline $\mathrm{C}-3 \mu$ emulsion & & 9 & & 0 \\
\hline $\mathrm{V}-4$ emulsion & 13 & & 2 & \\
\hline $\mathrm{V}-2 \mu$ counter & 16 & & 4 & \\
\hline $\mathrm{C}-3 \mu$ counter & & 4 & & 0 \\
\hline
\end{tabular}

Table 20: Wrong charge vertices in the Level 4 charm pairs.

\begin{tabular}{|l|c|c|c|c|}
\hline Topology & $Q= \pm 0$ & $Q= \pm 1$ & $Q= \pm 2$ & $Q= \pm 3$ \\
\hline V-2 emulsion & 21 & & 1 & \\
\hline V-2 $\mu$ emulsion & 20 & & 0 & \\
\hline C-3 emulsion & & 11 & & 0 \\
\hline C-3 $\mu$ emulsion & & 6 & & 0 \\
\hline V-4 emulsion & 7 & & 0 & \\
\hline V-2 $\mu$ counter & 10 & & 0 & \\
\hline $\mathrm{C}-3 \mu$ counter & & 2 & & 0 \\
\hline
\end{tabular}

Table 21: Wrong charge vertices in the Level 5 charm pairs. 


\begin{tabular}{|l|c|c|c|c|c|c|}
\hline Background & \multicolumn{2}{|c|}{$\begin{array}{c}\text { Complete } \\
\text { Data Set }\end{array}$} & \multicolumn{2}{c|}{ Level 4 } & \multicolumn{2}{c|}{ Level 5 } \\
\hline & $\begin{array}{c}\text { Signal + } \\
\text { Background }\end{array}$ & Bockgrumnd & $\begin{array}{c}\text { Signal + } \\
\text { Background }\end{array}$ & Background & $\begin{array}{c}\text { Signal + } \\
\text { Background }\end{array}$ & Background \\
\hline $\begin{array}{l}\text { Diffractive } \\
\text { Interactions } \\
\text { in Emulsion }\end{array}$ & 851 & $15.9 \pm 2.11$ & 167 & $3.43 \pm 0.53$ & 61 & $1.14 \pm 0.28$ \\
\hline $\begin{array}{l}\text { Wroug sign } \\
\text { analysis }\end{array}$ & 165 & $41 \pm 6.4$ & 63 & $4 \pm 2.0$ & 19 & $0.0_{-0.0}^{+1.0}$ \\
\hline $\begin{array}{l}\text { Wrong charge } \\
\text { analygis }\end{array}$ & 442 & $83 \pm 9.1$ & 124 & $9 \pm 3$ & 62 & $1.0 \pm 1.0$ \\
\hline \hline Total & & & 198 & $16.4 \pm 3.6$ & 74 & $2.1 \pm 1.0$ \\
\hline
\end{tabular}

Table 22: Total Background for the charm pairs. The diffractive interaction background is lower than measured due to additional emulsion measurements.

\subsection{Final Background Determination}

The previous sections described how the backgrounds were determined for the different topologies and gave results based on looking at the entire data sample and the charm pair samples of Level 4 and 5 . Table 22 shows the the final total background for the three different sources just described. The total non-charm background is estimated to be $2.1 \pm 1.0$ events, this estimate is conservative since it scales the diffractive background from the complete data set. Improvement of signal to background ratios at different cut levels demonstrates that the charm pair selection reduced the background to signal ratio, but no quantitative means were available to account for this reduction. The value of $2.1 \pm 1.0$ background events is taken to be an upper limit and is low enough to permit data analysis on the complet " charm pair data set without background subtraction. 


\section{Chapter 6}

\section{Momentum Estimation}

\subsection{Introduction}

The study of the production characteristics of charm pairs requires that the momentum of each of the two charm particles be known. Adding the two momentum vectors of the individual charm, yields the momentum for the charm pair system so the production kinematics and dynamics of the charm pair can be measured. The $\mu$ trigger used in E653 forced at least one of the two charm decays in an event to be semi-leptonic and therefore to have an undetected neutrino. The partner decay was unbiased, other than by dynamic production correlations, and had a neutral daughter $\sim 90 \%$ of the time. Although hadronic neutrals ( $\left.\pi^{\circ} s, K^{\circ} s . ..\right)$ could be detected using calorimeters, the efficiency for detecting these neutrals strongly limited the number of unbiased decays that could be used to complete a charm pair event.

A technique that estimated the charm momentum without measuring the momentum of 
the neutral daughters allowed production studies on a large sample of charm decays from the E653 data. The background in the charm sample must be small in order for the momentum estimator to work well. This chapter will describe the philosophy, development, and performance of the moinentum estimator used for production analysis of clarm singles and charm pairs.

\subsection{Kinematics}

The momentum estimator developed for the production studies was based on modeling the charm decay as a two body decay. One body was the vector sum of the visible (charged) momentum and the other body was the neutral momentum [24]. The neutral system was assigned enough momentum to balance the momentum perpendicular to the charm direction (P-perp, $P_{t}$ ). Figure 35 depicts the momentum vectors in the laboratory frame for a neutral decay and Figure 36 shows the momentum vectors in the center of mass frame. The model assumed that the daughter particles are produced having only transverse momentum in the COM frame with respect to the parent direction.

\subsubsection{Mass Assignments}

The boost that transformed the COM frame to the LAB frame was defined by $\gamma_{v i s} . \gamma_{v i s}$ was calculated by making mass assignments to the charged tracks, and calculating the $\gamma$ that boosted the visible momentum to the frame where the visible momentum along the part it direction, $P_{L v i s}$, equals zero. The mass assignments depended on whether the vertex in question had a muon attached (muonic vertex) or if it was an unbiased vertex 


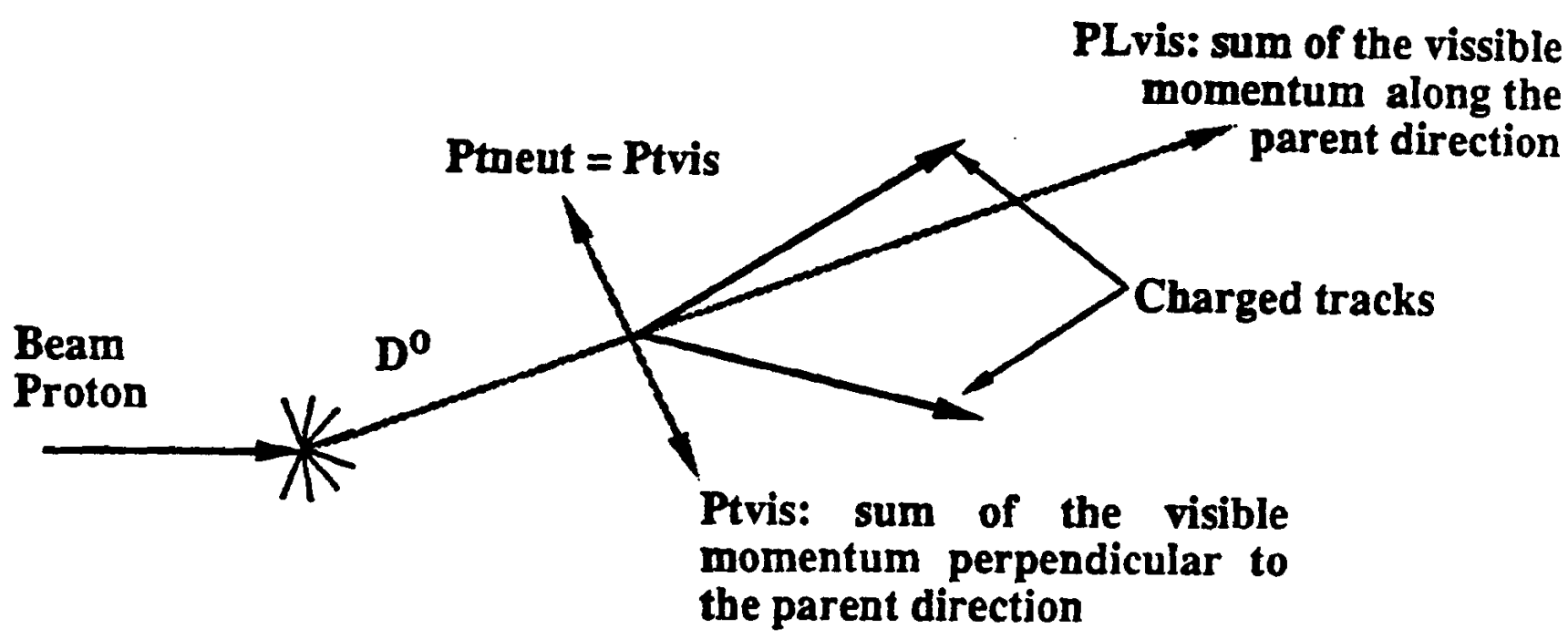

Figure 35: Momentum vectors in the laboratory frame for a neutral decay.

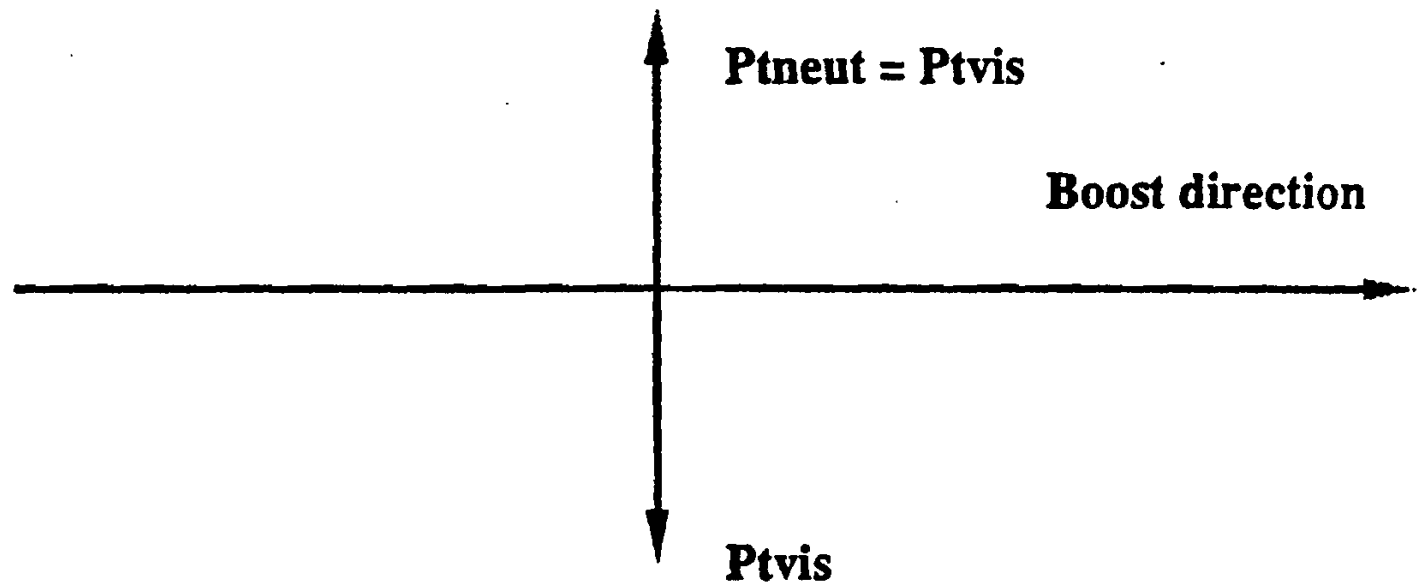

Figure 36: Momentum vectors in the center-of-mass (COM) frame for a neutral decay. Note that the om; momentum in the center-of-mass frame in perpendicular to the parent. direction in the LAB frame. 

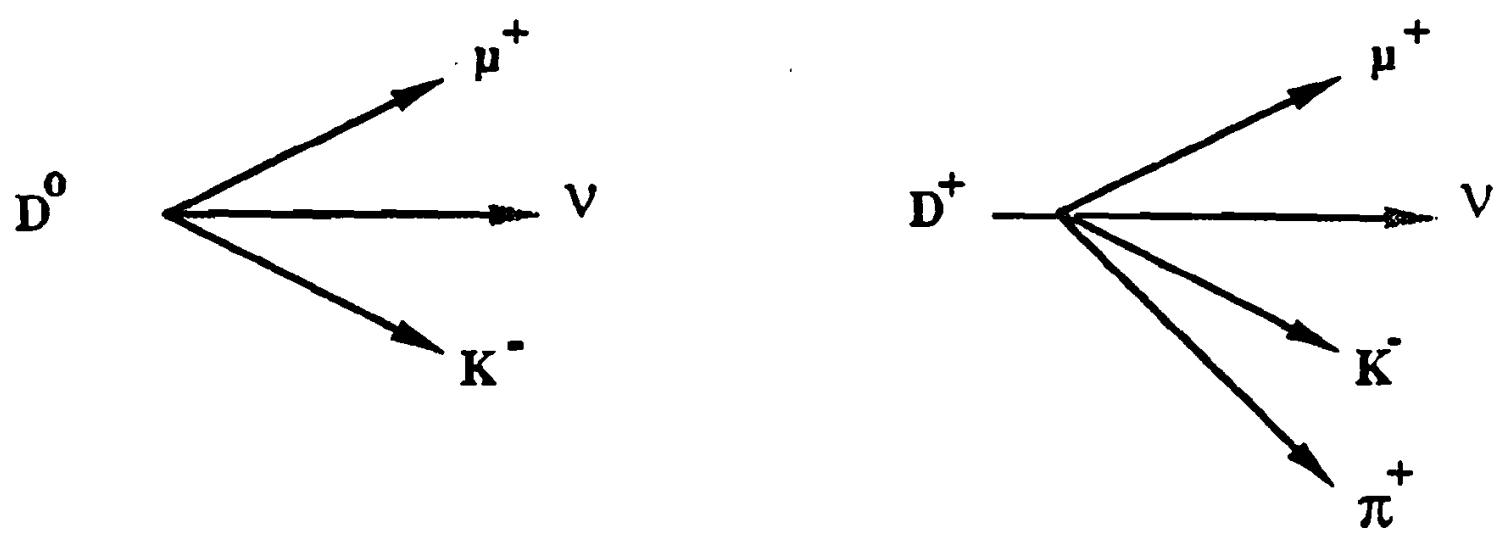

Figure 37: Mass assignments for decays with a tagged muon.

(hadronic vertex). For decays with a tagged muonic daughter, the muon track was assigned the muon mass and the track with charge opposite to that of the muon was assigned a kaon mass. For muonic tridents, the track with the same charge of the muon was assigned a $\pi$ mass. The missing neutral for muonic vertices was assumed to be a neutrino with zero mass. Figure 37 shows the mass assignments for neutral and charged muonic decays. Unbiased decays were assumed to be hadronic and the mass assignments depend on the the sign of the muon in the partner decay (Figure 38). The track with the same charge as the muon in the event was assigned a kaon mass and tracks opposite in charge with respect to the muon were assigned a $\pi$ mass. The missing neutral was E.sumed to be a $\pi^{\circ}$. For neuti.l decays with four charged tracks (V-4's) all the charged tracks were assumed to be $\pi^{\prime}$ s and the neutral track was a particle with zero mass. 

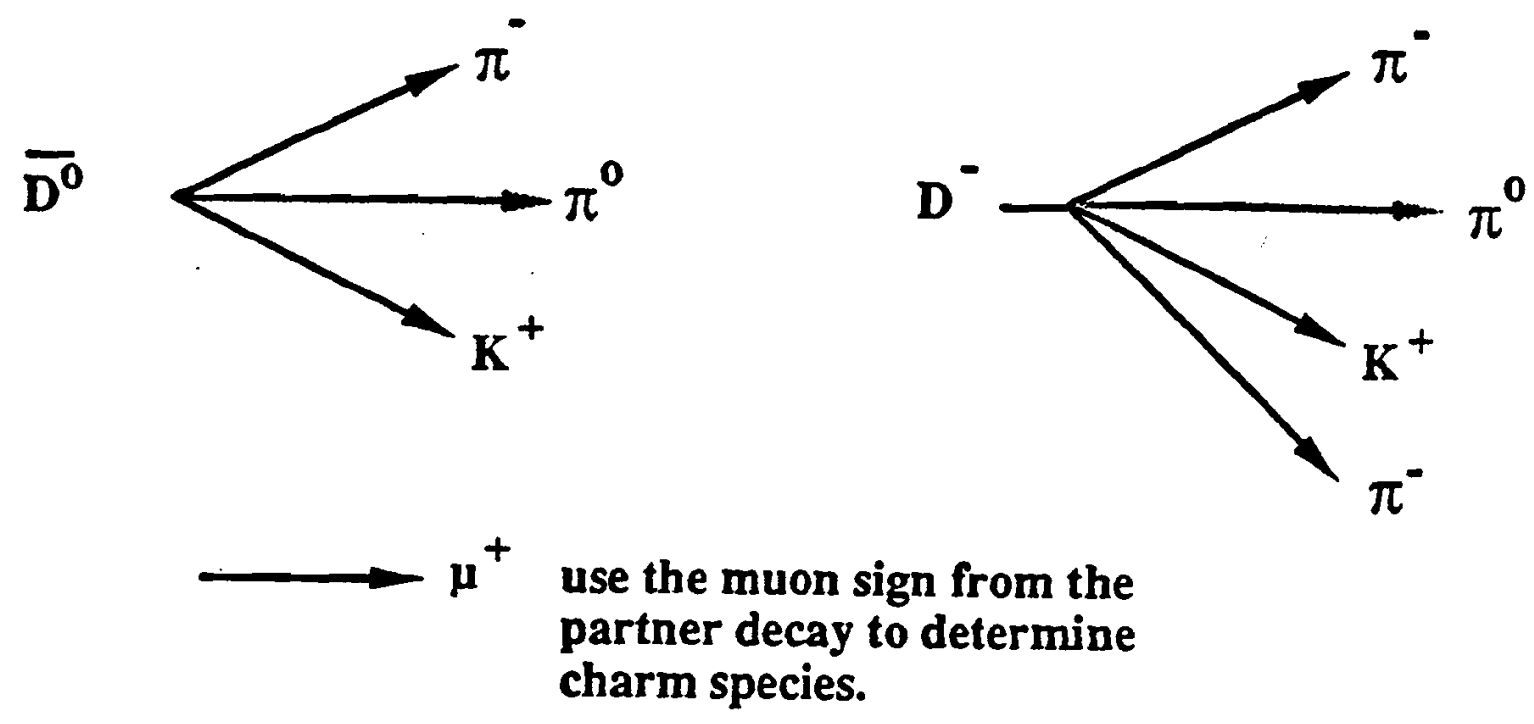

Figure 38: Mass assignments for decays without a tagged muon.

Once the mass assignments were given, the calculation for the momentum estimate was straight forward.

$$
\begin{aligned}
\gamma_{\text {vis }} & =E_{\text {Lvis }} / M_{\text {vis }} \\
E_{\text {Lvis }} & =\sqrt{P_{\text {Lvis }}^{2}+M_{v i s}^{2}} \\
M_{\text {vis }} & =\sqrt{E_{\text {vis }}^{2}-P_{v i s}^{2}} \\
P_{\text {tneut }} & =P_{\text {tvis }} \\
P_{\text {min }} & =M_{D} * \gamma_{v i s}
\end{aligned}
$$




$$
M_{\min }=\sqrt{P_{\text {tvis }}^{2}+M_{v i s}^{2}}+\sqrt{P_{\text {treut }}^{2}+M_{\text {neut }}^{2}}
$$

$M_{\min }$ was the transverse mass for the decay and the closer $M_{\min }$ was to the actual value of $M_{D}$, the more accurate the momentum estimate $\left(P_{\min }\right)$. Using Monte Carlo generated charm decays the resolution function for the momentum estimate was parameterized as a function of $M_{\min }$.

\subsection{The Resolution Function and Monte Carlo Studies}

Moute Carlo decays of charmed meson $\left(D^{\circ}, D^{+}\right)$into Caḅbibo favored modes were generated to develop the momentun estimator. The decays in the E653 data sample were divided into distinct topologies by the prong count and muon tag. The dominant and measured decay modes were installed into the Monte Carlo so that a specific topology could be studied. The decay modes for the topologies used in production studies are shown in Table 23 with the branching ratios that were used in this analysis. 


\begin{tabular}{|c|c|c|}
\hline Mode & Reference & Branching Ratio (percent) \\
\hline \multicolumn{3}{|l|}{ Hadronic Kinks } \\
\hline$D^{+} \rightarrow K^{\circ} \pi^{+}$ & [38] & $2.8 \pm 0.4$ \\
\hline$D^{+} \rightarrow K^{0} \pi^{+} \pi^{0}$ & 38 & $8.3 \pm 1.8$ \\
\hline$D^{+} \rightarrow K^{0} \pi^{+} \pi^{0} \pi^{0}$ & [61] & 2.8 \\
\hline \multicolumn{3}{|l|}{ Muonic Kinks } \\
\hline$D^{+} \rightarrow K^{\circ} \mu^{+} \nu$ & [60] & $10.2 \pm 3.1 \pm 1.7$ \\
\hline$D^{+} \rightarrow K^{\circ} \pi^{\circ} \mu^{+} \nu$ & [60] & $3.8 \pm_{1 \tau}^{5.3} \pm 0.6$ \\
\hline \multicolumn{3}{|l|}{ Hadronic V-2's } \\
\hline$D^{\circ} \rightarrow K^{-} \pi^{+}$ & [38] & $3.77 \pm_{032}^{0.37}$ \\
\hline$D^{\circ} \rightarrow K^{-} \pi^{+} \pi^{o}$ & 38 & $12.5 \pm_{14}^{1.5}$ \\
\hline$D^{\circ} \rightarrow K^{\circ} \pi^{+} \pi^{+}$ & [38] & $5.6 \pm_{0 .}^{0 . t}$ \\
\hline$D^{\circ} \rightarrow K^{-} \pi^{+} \pi^{0} \pi^{0}$ & 38 & $15 \pm 5$ \\
\hline$D^{\circ} \rightarrow K^{\circ} \pi^{+} \pi^{-} \pi^{0}$ & [37] & $11.5 \pm 2.2 \pm 2.8$ \\
\hline \multicolumn{3}{|l|}{ Muonic V-2's } \\
\hline$D^{\circ} \rightarrow K^{-} \mu^{+} \nu$ & [37] & $4.1 \pm 0.1 \pm 1.2$ \\
\hline$D^{\circ} \rightarrow K^{\circ} \pi^{-} \mu^{+} \nu$ & [37] & $2.7 \pm 1.1 \pm 1.6$ \\
\hline$D^{0} \rightarrow K^{-} \pi^{0} \mu^{+} \nu$ & [37] & $1.7 \pm 0.9 \pm 0.6$ \\
\hline \multicolumn{3}{|l|}{ Hadronic C-3's } \\
\hline$D^{+} \rightarrow K^{-} \pi^{+} \pi^{+}$ & [38] & $7.8 \pm_{0.8}^{1.1}$ \\
\hline$D^{+} \rightarrow K^{-} \pi^{+} \pi^{+} \pi^{0}$ & [38] & $3.7 \pm_{0.8}^{1.5}$ \\
\hline$D^{+} \rightarrow K^{-0} \pi^{-} \pi^{+} \pi^{+}$ & [38] & $7.0 \pm \pm_{21}^{2.3}$ \\
\hline$D^{+} \rightarrow K^{-} \pi^{+} \pi^{+} \pi^{0} \pi^{0}$ & [54] & $2.2 \pm \frac{5.0}{5.0}$ \\
\hline$D^{+} \rightarrow K^{\circ} \pi^{-} \pi^{+} \pi^{+} \pi^{\circ}$ & [54] & $4.4 \pm_{n 7}^{5.0}$ \\
\hline \multicolumn{3}{|l|}{ Muonic C-3's } \\
\hline$D^{+} \rightarrow K^{-} \pi^{+} \mu^{+} \nu$ & [37] & $3.9 \pm 0.9 \pm 0.7$ \\
\hline$D^{+} \rightarrow K^{0} \pi^{+} \pi^{-} \mu^{+} \nu$ & [38] & $2.2 \pm_{07}^{5.0}$ \\
\hline$D^{+} \rightarrow K^{-} \pi^{+} \pi^{0} \mu^{+} \nu$ & 38 & $4.4 \pm_{1.5}^{5.2}$ \\
\hline \multicolumn{3}{|l|}{ Hadronic V-4's } \\
\hline$D^{\circ} \rightarrow K^{-} \pi^{-} \pi^{+} \pi^{+}$ & [38] & $7.9 \pm 0.1 .0$ \\
\hline$D^{0} \rightarrow K^{0} \pi^{-} \pi^{-} \pi^{+} \pi^{+}$ & [61] & 4.3 \\
\hline$D^{\circ} \rightarrow K^{-} \pi^{-} \pi^{+} \pi^{+} \pi^{\circ}$ & [61] & 7.9 \\
\hline
\end{tabular}

Table 23: Branching Ratios used in the Monte Carlo development of the momentum estimator and charm selection. 
The generated momentum was then compared to the momentum estimate of $P_{\min }$ as a function of $M_{\min }$. Figure 40 shows the scatter plot of $P_{\min } / P_{\text {generated }}$ versus $M_{\min }$ for $D^{\circ}$ two prong decays. An important feature of Figure 40 is that the $M_{\min }$ distribution is concentrated at the value for the D mass. This means that the estimator performed well for the majority of the decays. The resolution dependence on $M_{\min }$ is also quite evident from Figure 40 and the projections onto the $P_{\min } / P_{\text {gen }}$ axis for different slices in $M_{\min }$ shown in Figure 41.

$M_{\min }$ was a measured quantity and had measurement errors (assumed to be gaussian). The resolution distribution that corresponded to a measured value for $M_{\min }$ and its error was the sum of the resolution function distributions weighted by the amount defined by the value for $M_{\min }$ and its error (Equation 34). (Error in $M_{\min }$ depends on the error in the $P_{t}$ of the decay.)

$$
\begin{aligned}
M_{\min } & =M_{\min } \pm \delta M_{\min } \\
\text { Resolution Function } & =\sum R_{j} \int_{m_{0 j}}^{m_{f j}}\left(\sqrt{2 \pi} \delta M_{\min }\right)^{-1} e^{\left(m_{j}-M_{\min }\right)^{2} / \delta M_{\min }{ }^{2} d m_{j}} \\
R_{j} & =P_{\min } / P_{\text {gen }} \text { distribution for } m_{0 j}<m_{j}<m_{f j}
\end{aligned}
$$

For example: If $M_{\min }$ and its error were entirely within a $100 \mathrm{MeV} / \mathrm{c}^{2}$ interval in $M_{\min }$, the resolution function representing that value of $M_{\min }$ would be assigned to the decay. If $M_{\min }$ and its error were situated $50 \%$ in $\mathrm{R}_{j}$ and $: 0 \%$ in $\mathrm{R}_{i}$, the resolution function, $\mathrm{R}$, would be the average of the two resolution functions, $R=\left(R_{j}+R_{i}\right) / 2$. The integral in 
Equation 34 represents the weight assigned to the $100 \mathrm{MeV} / \mathrm{c}^{2}$ binned resolution functions. The resolution function for the decay was then shifted so the average value for $P_{\min } / P_{\text {generated }}$ is equal to one. Figures 42 and 43 are the projected resolution distributions for hadronic $D^{\circ}$ 2-prong decays and Figures 44 and 45 are for muonic $D^{+}$ decays into three prongs. Figures 46 and 47 show the resolution distributions for hadronic three prong $D^{+}$decays and Figures 48 and 49 are for four prong hadrouic $D^{\circ}$ decays.

\subsection{The 0-C Analogy}

The kinematics described in the previous section are analogous to those used for a $0-\mathrm{C}$ (unconstrained) fit to the decay. Using the same particle identification as in the previous section, Formulas 36 through 42 for the neutral momentum assuming a known parent mass are obtained. Equation 36 is a quadratic and will yield two solutions for the neutral momentum, except in the degenerate case. The two solutions represent the ambiguity in the longitudinal momentum in the COM frame. The degenerate solution was called the solution at the minimum since it represented the minimum of the curve shown in Figure 39 and it also represented the case where $P_{L}$ was equal to zero in the COM frame (This is the same assumption that was made in the previous section).

When this minimum was at the $\mathrm{D}$ mass the decay had only one momentum solution for the charm parent. For most charm decays the $\mathrm{D}$ mass was above the minimum mass and the solution at the minimum now represents the momentum for a parent particle with a mass equal to the minimum mass. The curve in Figure 39 shows the relation between the 
parent mass for the decay and its quadratic solutions for the neutral momentum. The closer the minimum mass was to the $\mathrm{D}$ mass the closer the momentun at the minimum was to the actual charm momentum.

Since the momentum estimator was first developed using the $0-\mathrm{C}$ techuique, the names for the momentum and mass variables were taken from this analogy. Hence $P_{\min }$ was the momentum for a particle of mass $M_{\text {min }}$ which decayed into the hypothesis chosen for this decay, or $M_{\min }$ and $P_{\min }$ were the mass and momentum for the decay if its daughters had only transverse momentum in the COM frame.

$$
\begin{aligned}
P_{\text {lneut }} & =-b / 2 a \pm \sqrt{b^{2}-4 a c} / 2 a \\
a & =P_{\text {lvis }}^{2} / E_{\text {vis }}^{2}-1 \\
b & =\left(M_{D}^{2}-M_{\text {vis }}^{2}-M_{\text {neut }}^{2}-2 P_{\text {tvis }}^{2}\right) P_{\text {lvis }} / E_{\text {vis }}^{2} \\
c & =\left(M_{D}^{2}-M_{\text {vis }}^{2}-M_{\text {neut }}^{2}-2 P_{\text {tvis }}^{2}\right)^{2} / 4 E_{\text {vis }}^{2}-P_{\text {tvis }}^{2}-M_{\text {neut }}^{2} \\
P_{D} & =P_{\text {lvis }}+P_{\text {lneut }} \\
M_{\min }^{2} & =\sqrt{4\left(P_{\text {tvis }}^{2}+M_{\text {neut }}^{2}\right) *\left(P_{\text {tvis }}^{2}+M_{v i s}^{2}\right)}+M_{\text {vis }}^{2}+M_{\text {neut }}^{2}+2 P_{\text {tvis }}^{2} \\
P_{\text {min }} & =-b / 2 a \text { with } M_{D}=M_{\text {min }} \\
P_{\min } & =P_{l v i s}+P_{l \text { min }}
\end{aligned}
$$




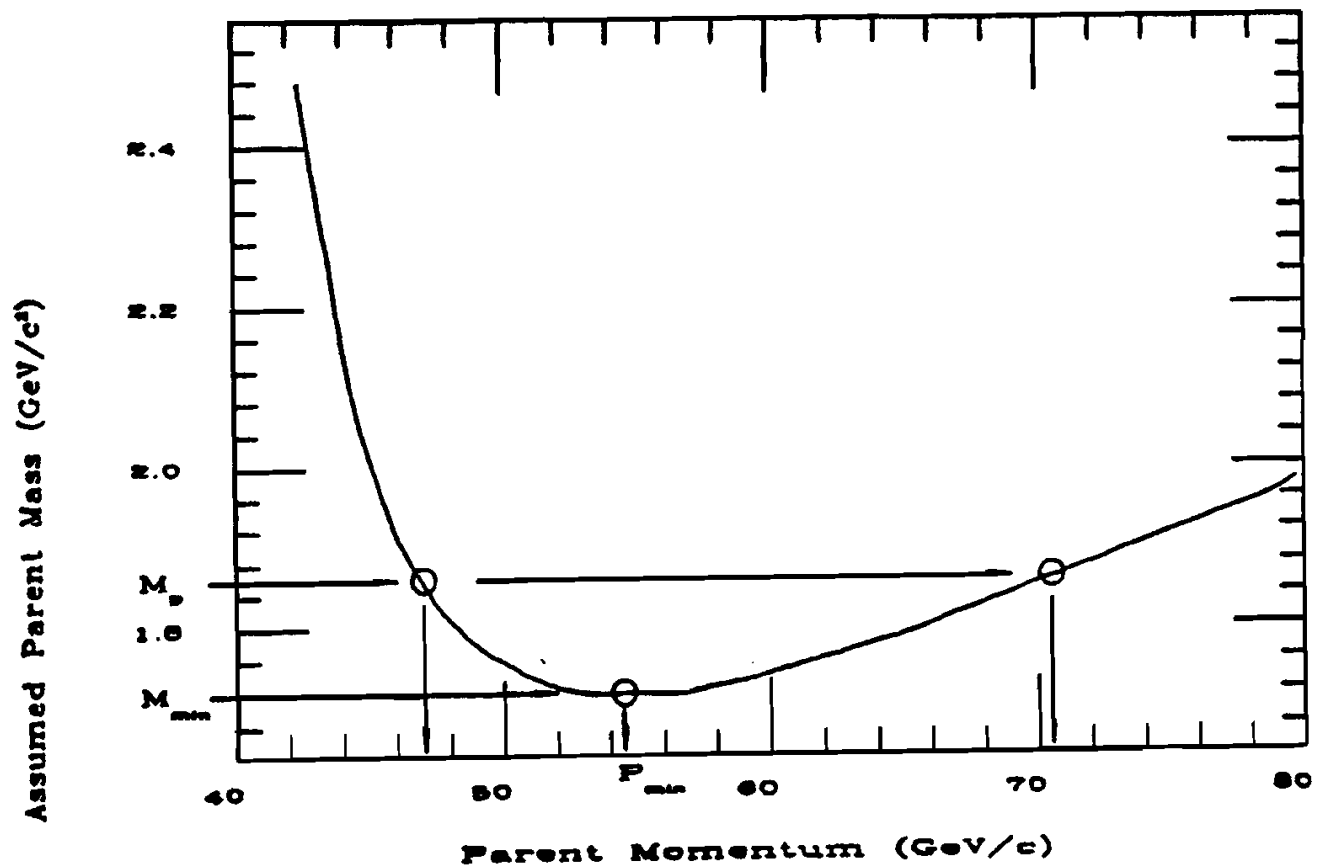

Figure 39: The momentum solutions for the $0-C$ equation as a function of parent mass.

\subsection{Other Charm Decay Modes}

The decay modes listed in Table 23 are not complete. However the performance of the estimator will not be strongly effected by the decay modes that are not accounted for. The resolution of the momentum estimator depends mainly on how many neutrals are involved in the decay, it does not depend heavily on the masses of the particles involved. This was seen by looking at neutral hadronic decays and studying the difference between the decays which involve $K^{\circ}$ 's and $\pi^{\circ}$ 's. The plots of the resolution function versus $M_{\min }$ are quite similar for the two sets of decays, and the fact that the kaon was mis-identified in the $K^{-0}$ decays has not resulted in any aignificant difference in the resolution functions. The momentum estimator will be tested at the end of the next section by comparing the results of maximum likelihooa fits on Monte Carlo data to the known generated values. 


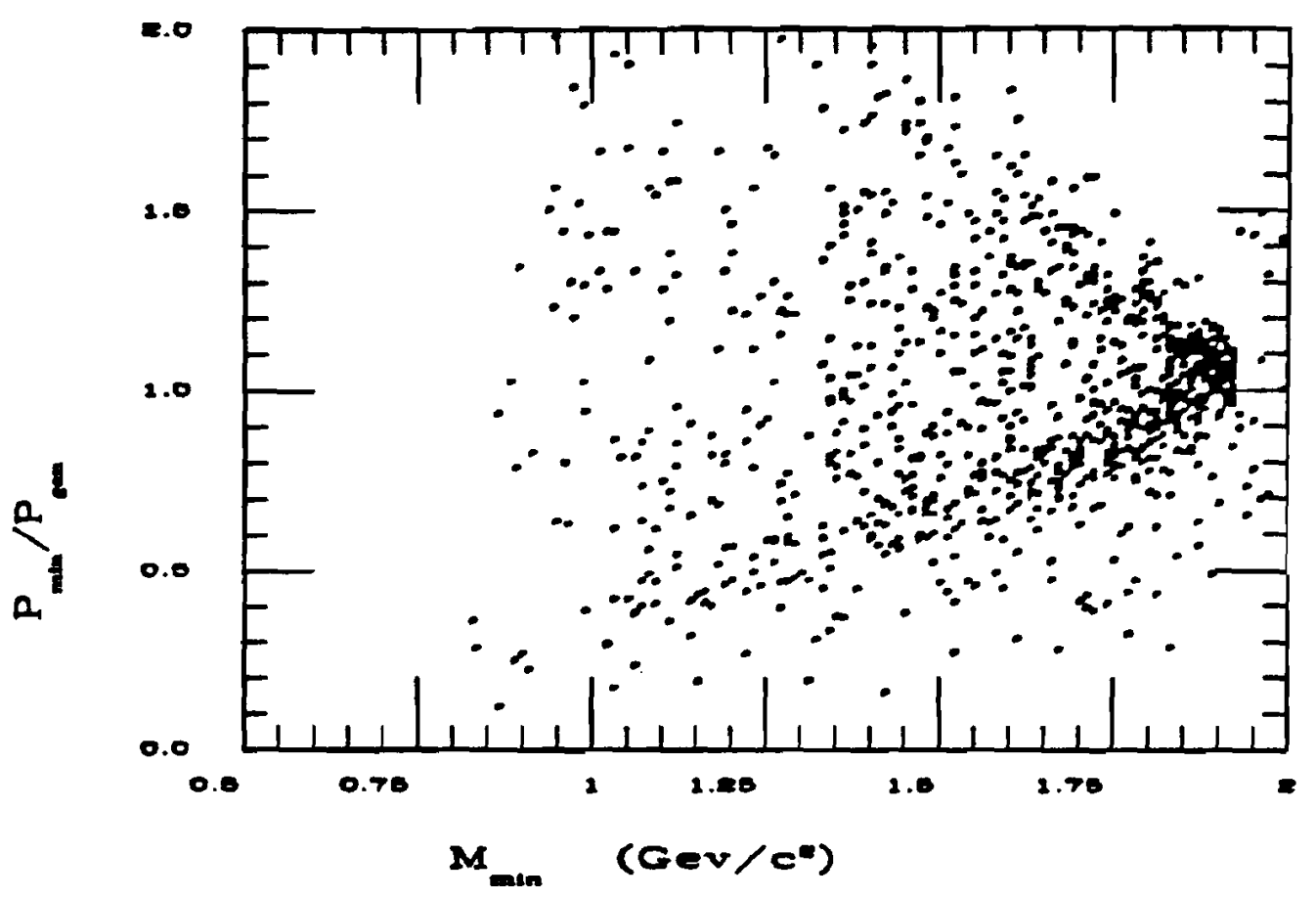

Figure 40: $P_{\min } / P_{\text {penerated }}$ versus $M_{\min }$ for two prong muonic $D^{\circ}$ decays. 

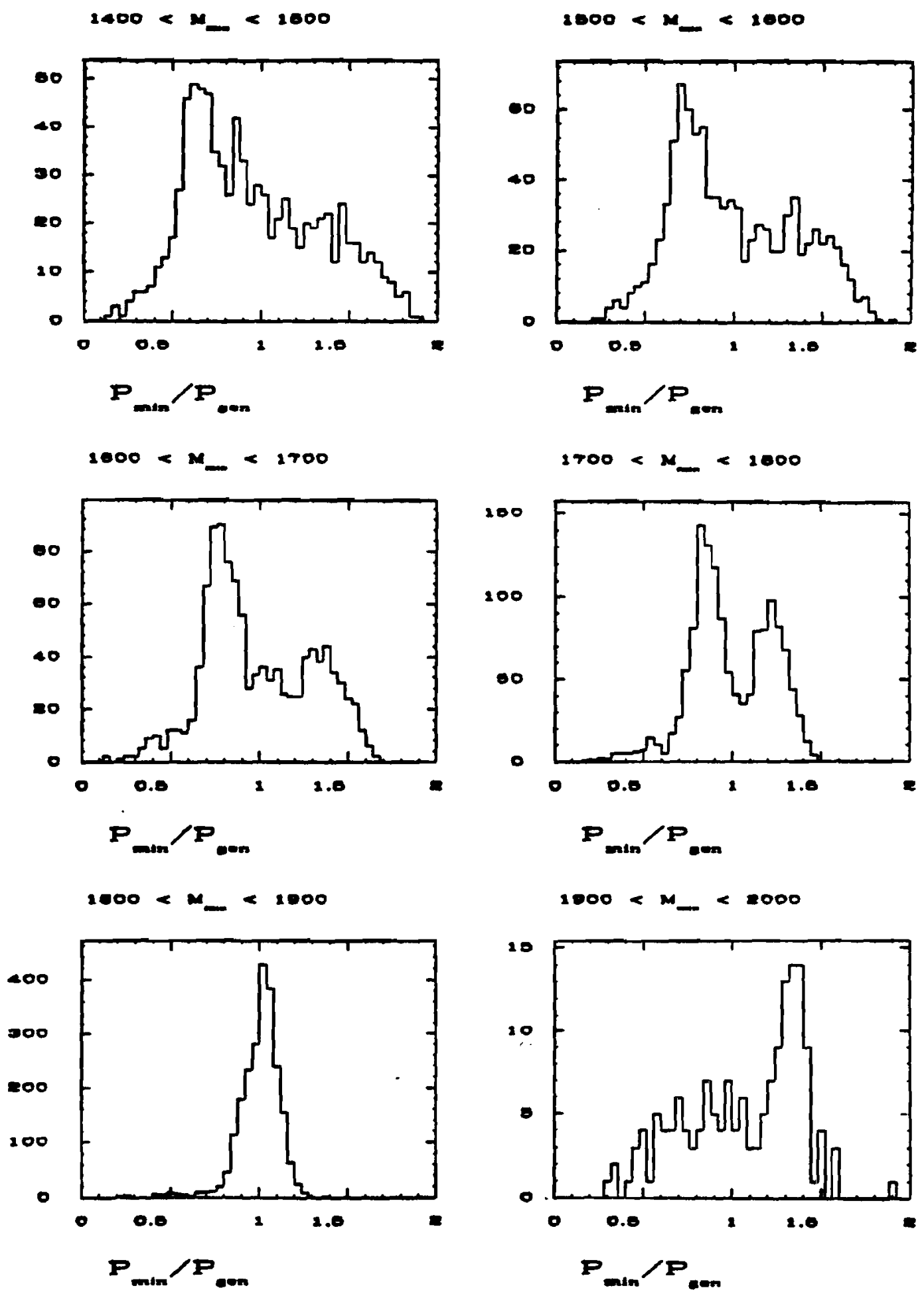

Figure 41: $P_{\min } / P_{\text {generated }}$ projections in $100 \mathrm{MeV} / \mathrm{c}^{2}$ slices of $M_{\min }$ for two prong muonic $D^{\circ}$ decays. 


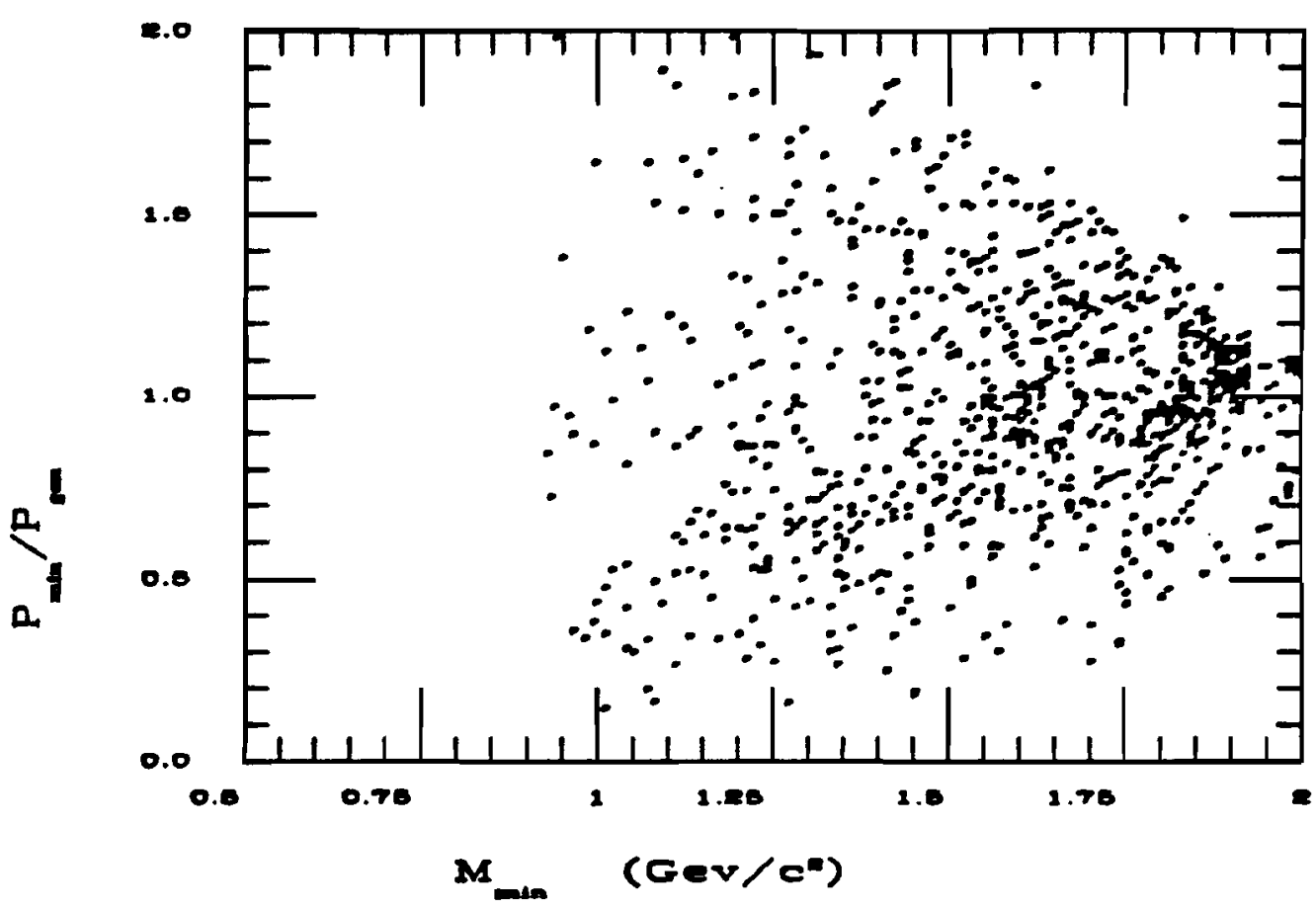

Figure 42: $P_{\min } / P_{\text {oenerated versus }} M_{\min }$ for two prong hadronic $D^{\circ}$ decays. 

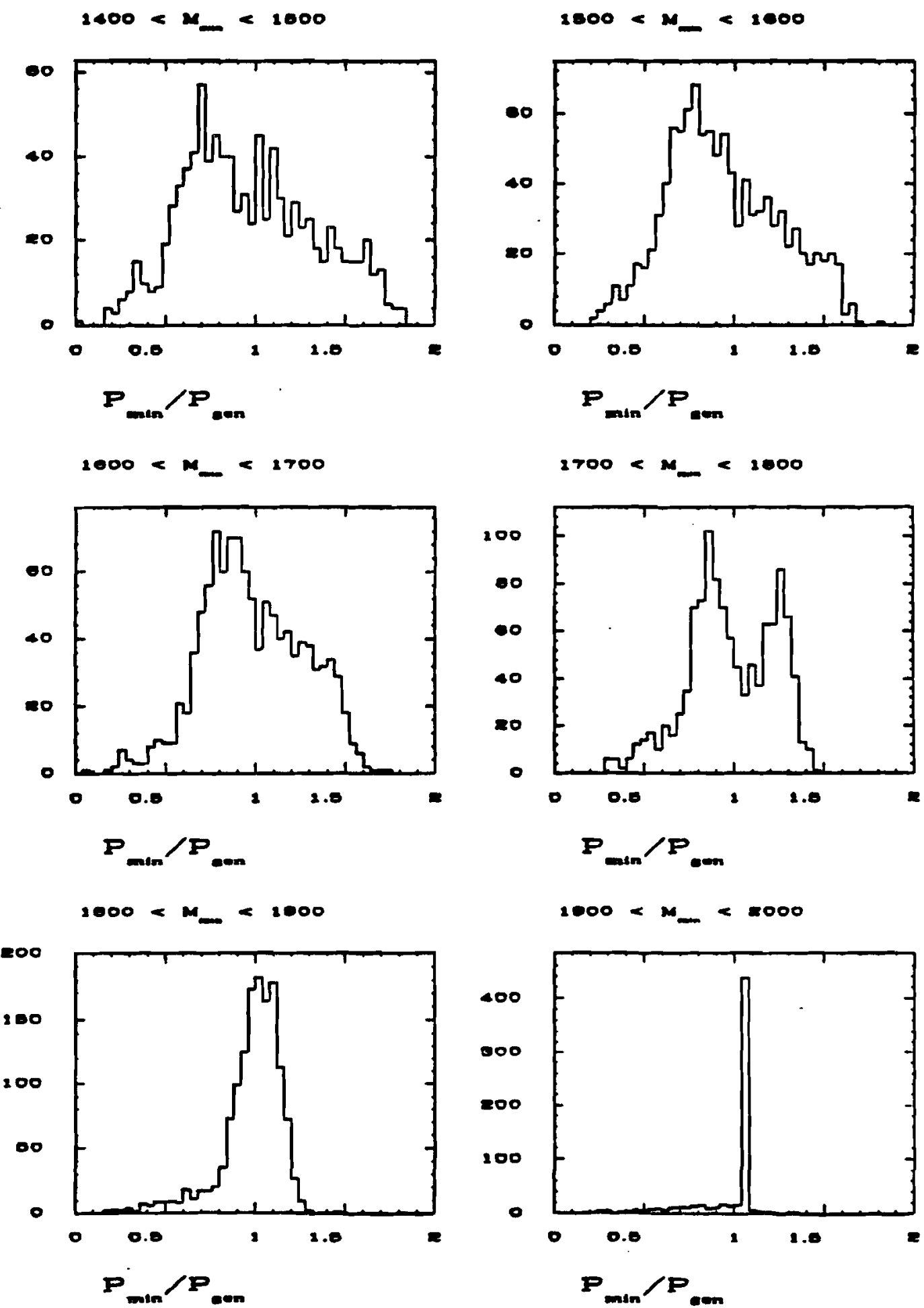

Figure 43: $P_{\min } / P_{\text {generated }}$ projections $100 \mathrm{MeV} / \mathrm{c}^{2}$ slices of $M_{\min }$ for two proug hadronic $D^{\circ}$ decays. 


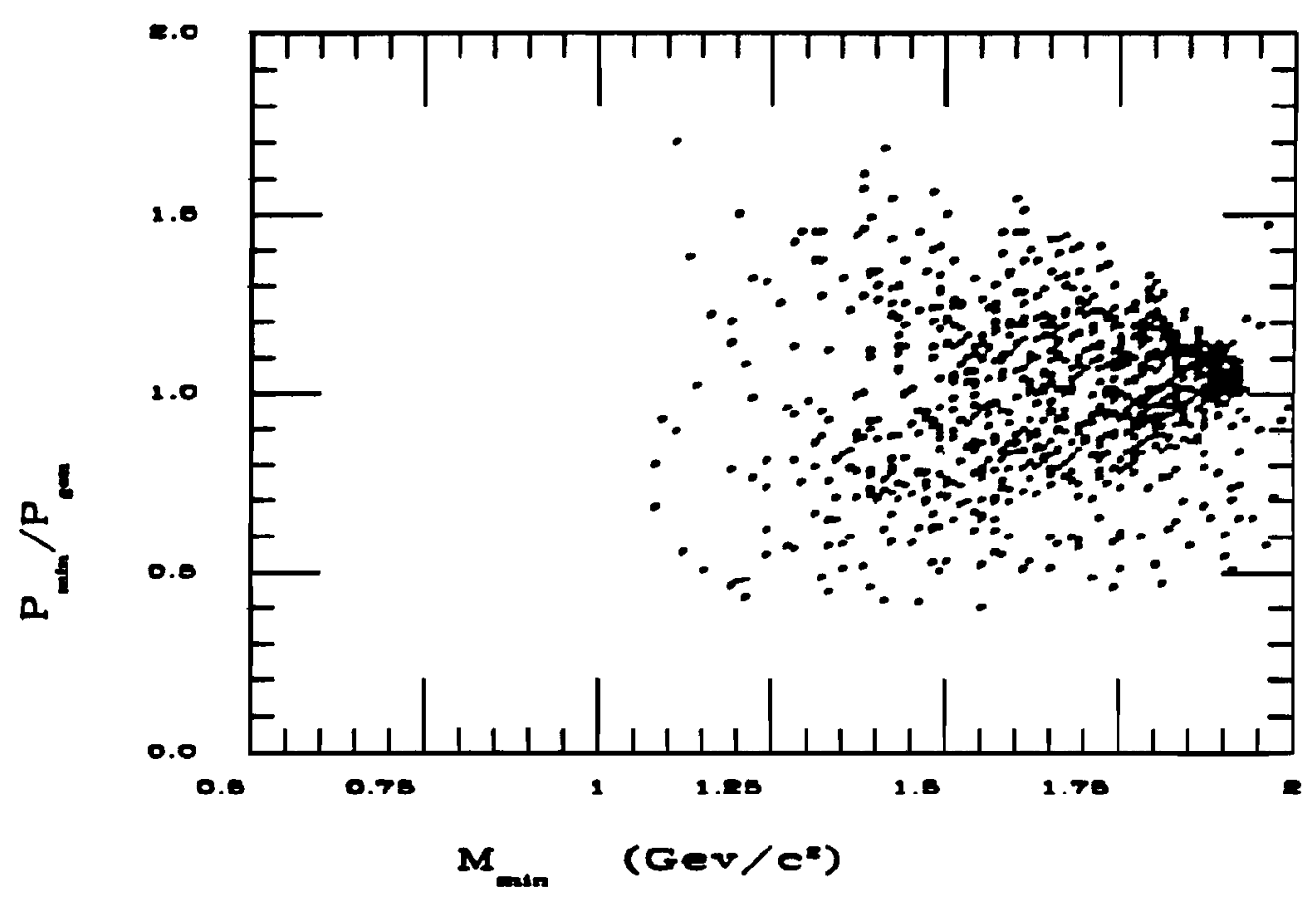

Figure 44: $P_{\min } / P_{\text {generated }}$ versus $M_{\min }$ for three prong muonic $D^{+}$decays. 

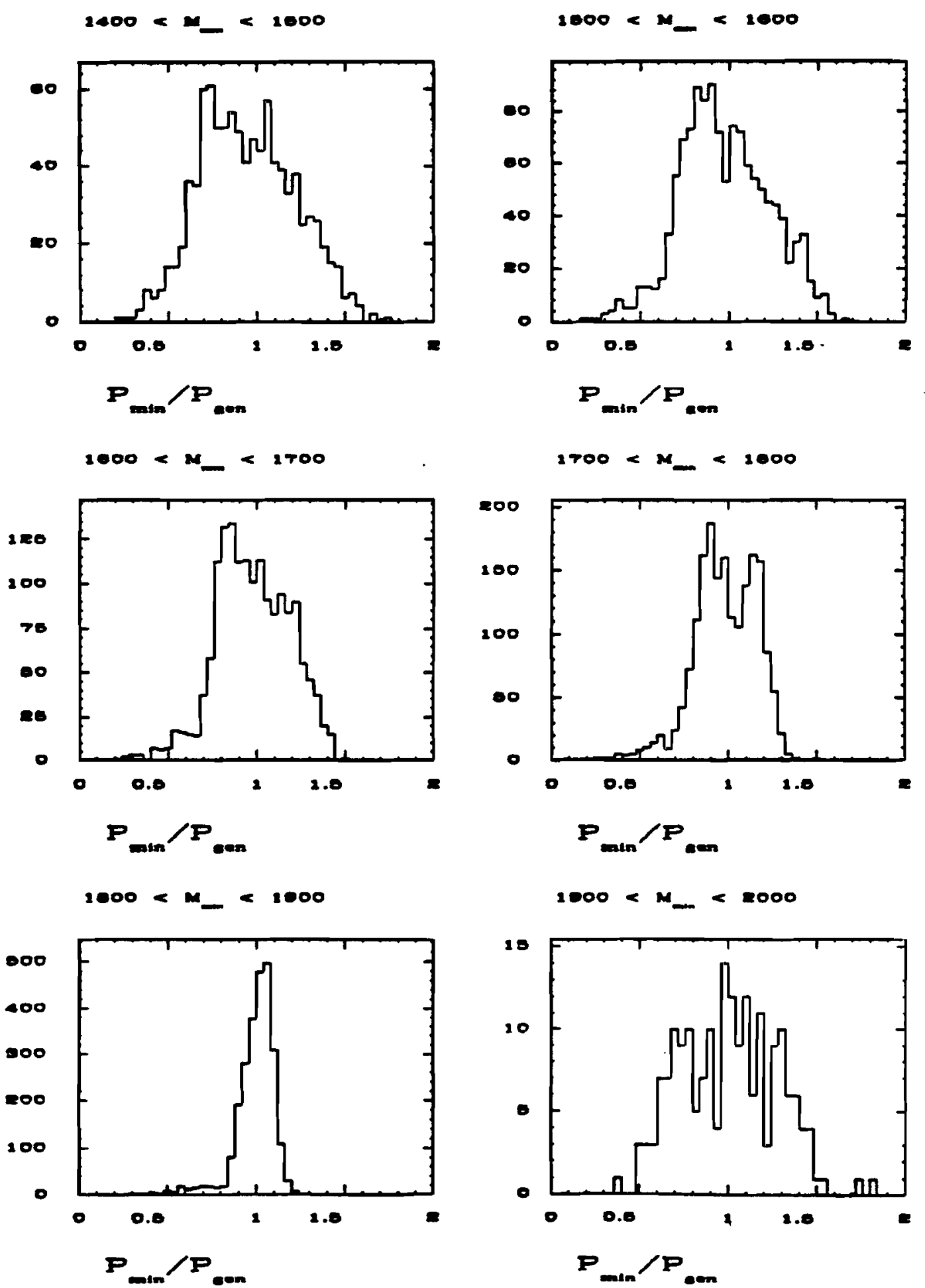

Figure 45: $P_{\min } / P_{\text {penerated }}$ projections in $100 \mathrm{MeV} / \mathrm{C}^{2}$ slices of $M_{\min }$ for three prong muonic $D^{+}$decays. 


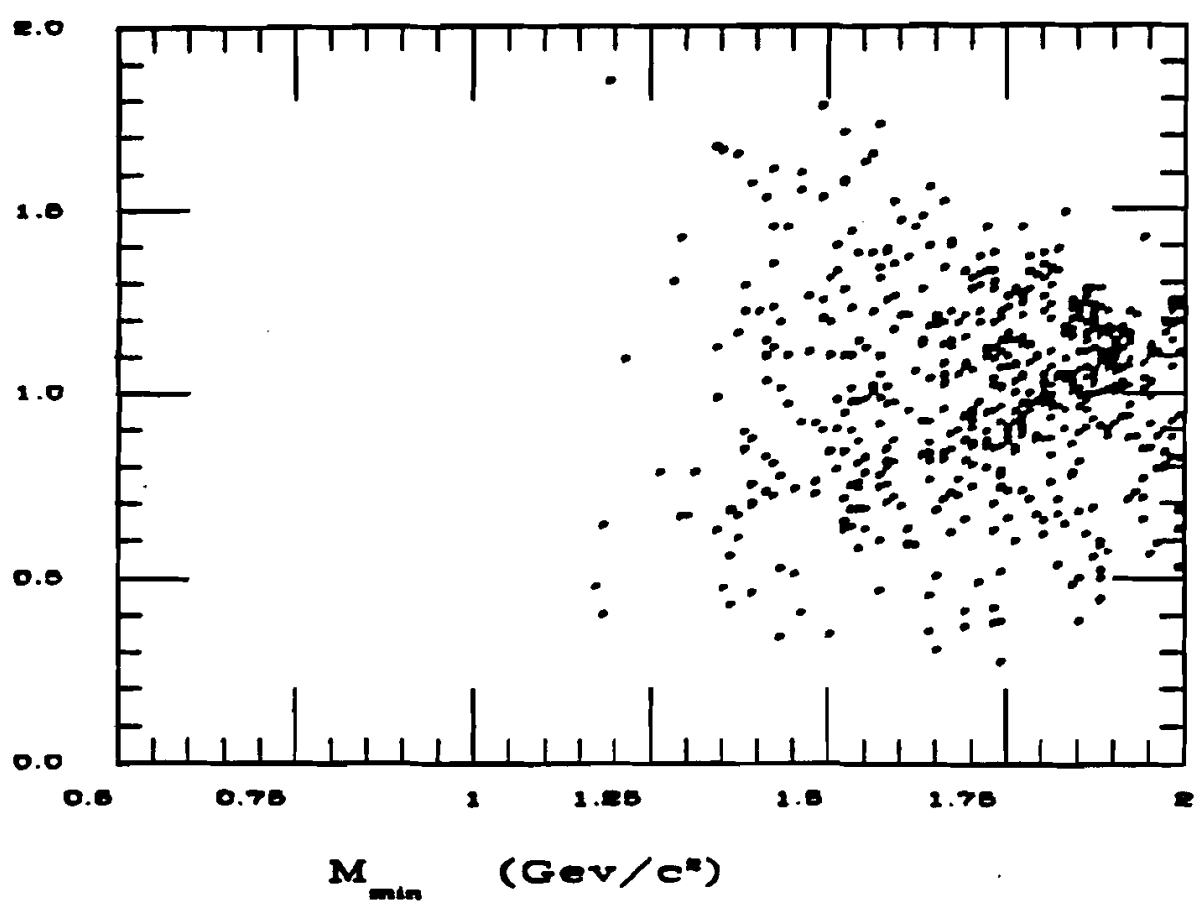

Figure 46: $P_{\min } / P_{\text {generated }}$ versus $M_{\min }$ for three prong hadrouic $D^{+}$decays. 

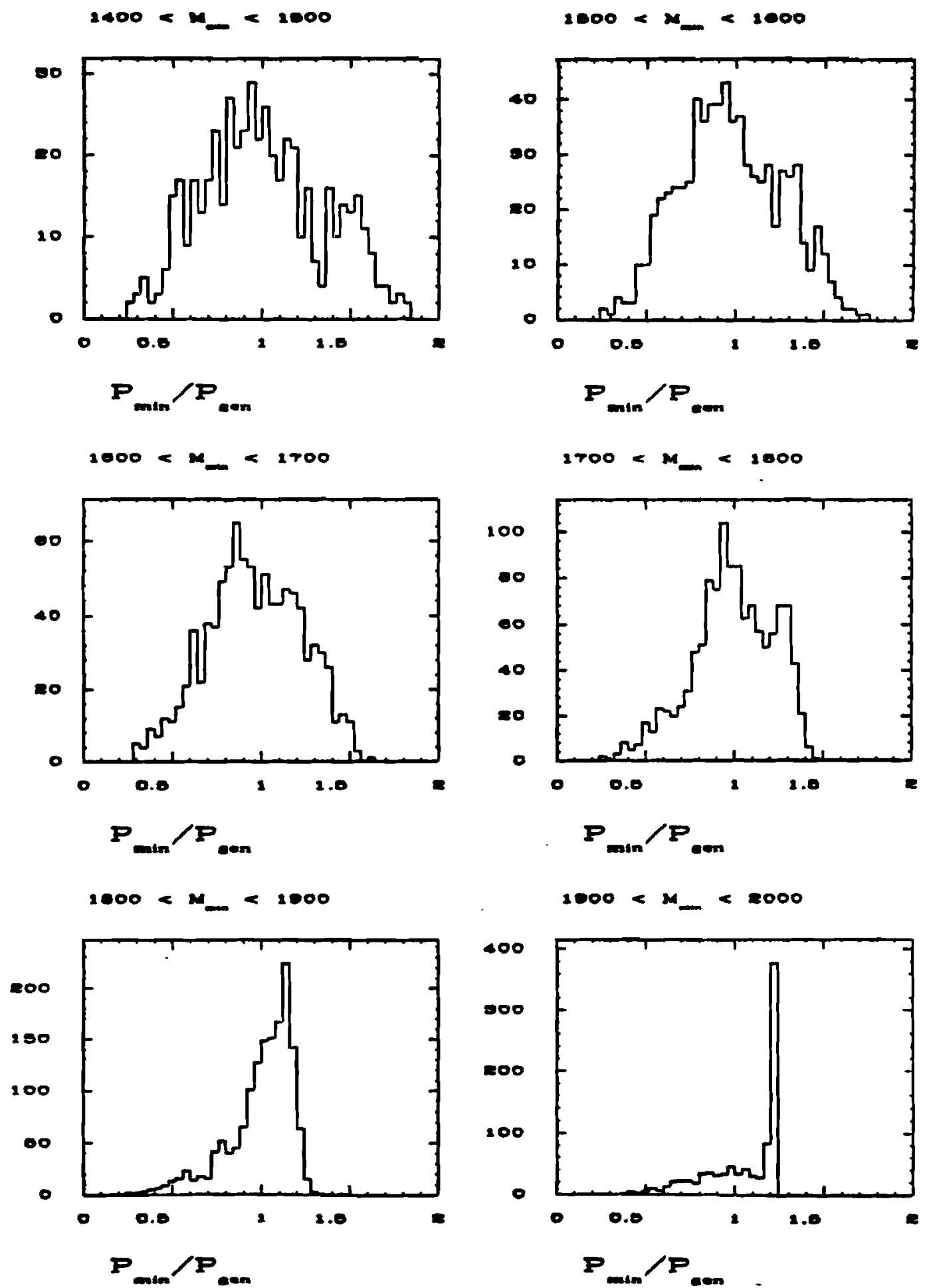

Figire 47: $P_{\min } / P_{\text {penerated }}$ projections in $100 \mathrm{MeV} / \mathrm{c}^{2}$ slices of $M_{\min }$ for three prong hadronic $D^{+}$decays. 


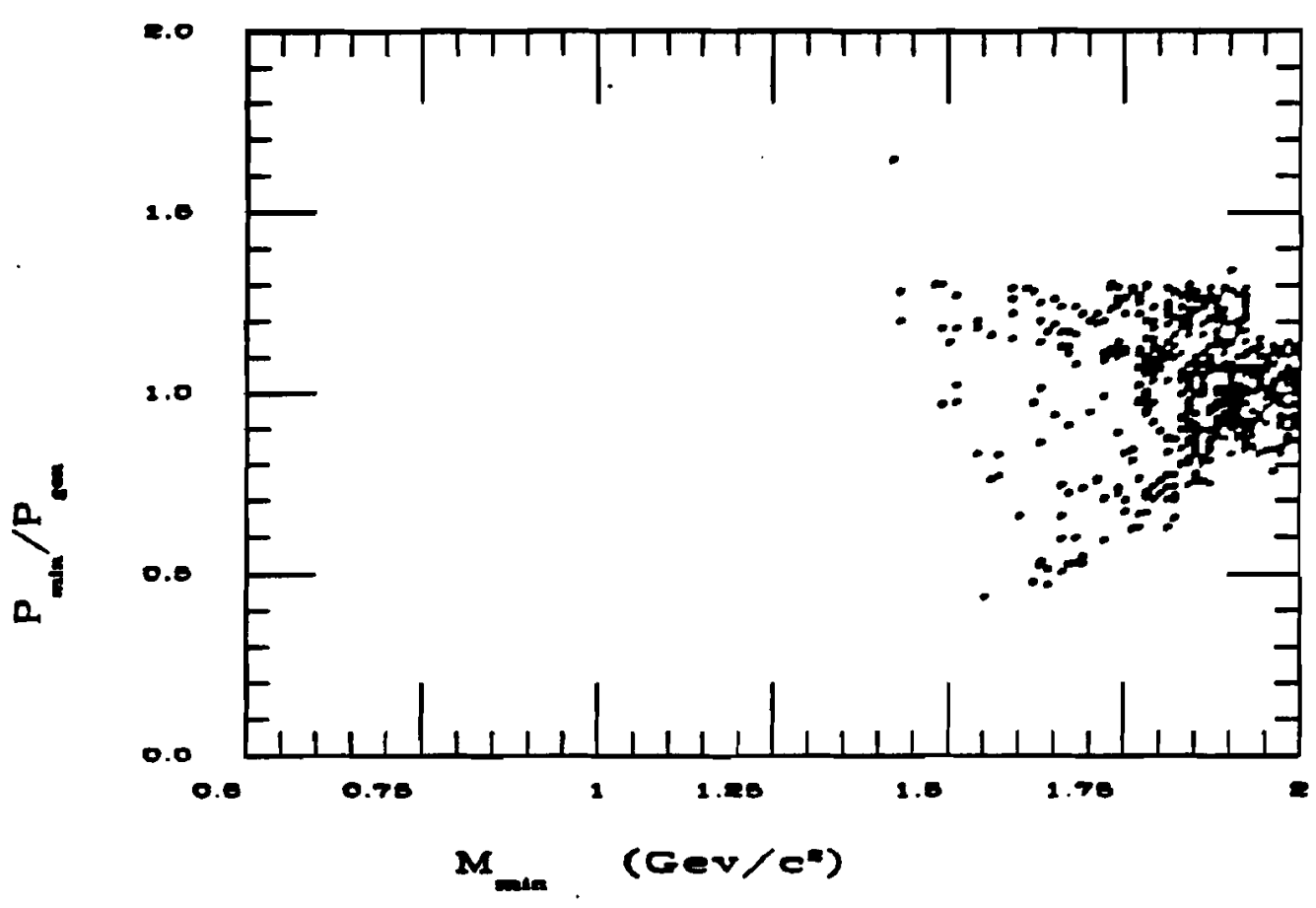

Figure 48: $P_{\min } / P_{\text {generated }}$ versus $M_{\min }$ for four prong hadronic $D^{\circ}$ decays. 

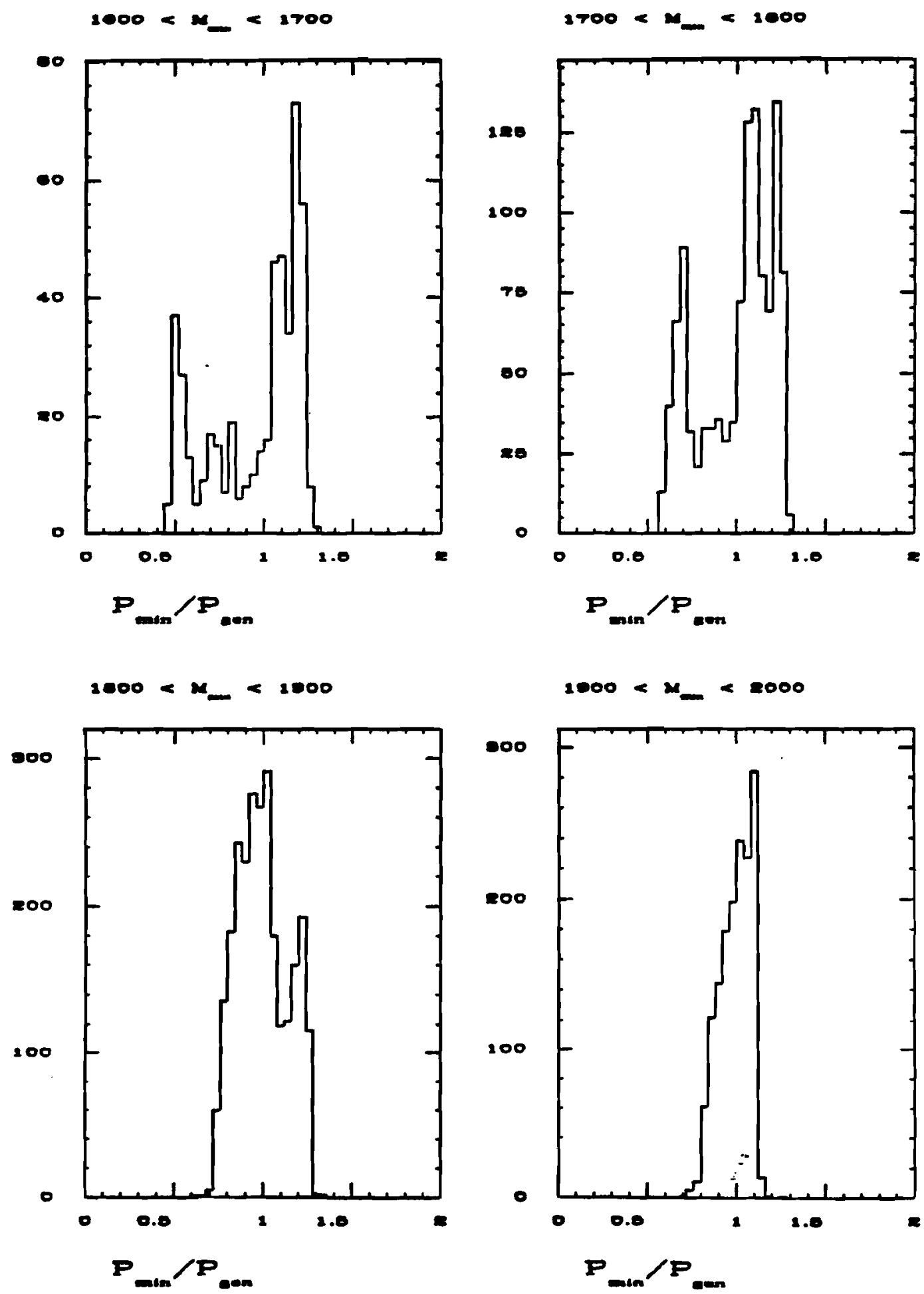

Figure 49: $P_{\min } / P_{\text {oenerated }}$ projections in $100 \mathrm{MeV} / \mathrm{c}^{2}$ slices of $M_{\min }$ for four proug hadronic $D^{\circ}$ decays. 


\section{Chapter 7}

\section{Maximum Likelihood Technique}

\section{and Efficiencies}

\subsection{Introduction}

The non-gaussian resolution function for the charm parent momentum introduced in the preceding chapter must be incorporated into the fitting analysis. Maximum likelihood fitting provided a natural method of incorporating generic resolution functions into the fitting procedure. This chapter will describe the maximum likelihood fitting technique used in E653 analysis. The first section will present a short review of the method, and the following sections will be devoted to its use in this analysis. 


\subsection{Probabilistic Motivation}

The maximum likelihood technique has been used in situations where due to low statistics least squares fitting was not applicable $[23,59]$. Since the technique relies on maximizing probabilities, incorporating a resolution function (or in other words a probability distribution) is straight forward.

The simplest situation for maximum likelihood fitting (Maxlike) is one where the resolution function is a $\delta$ function and the efficiency distribution is a constant. For this case, the probability of observing event $i$ as a function of $f\left(x_{i}, n\right)$ is:

$$
P_{i}\left(x_{i}, n\right)=N(n) f\left(x_{i}, n\right)
$$

where $x_{i}$ is the measured quantity and $n$ is the parameter to be determined. The probability distribution must be properly normalized such that:

$$
\int P_{i}\left(x_{i}, n\right) d x=1
$$

The likelihood distribution for the set of $i=(1,2, \ldots, m)$ events is

$$
\mathcal{L}(n)=\prod_{i=1}^{m} P_{i}\left(x_{i}, n\right)
$$

By maximizing $\mathcal{L}(n)$ with respect to $n$ the value for the $n$ that has the highest probability of describing the distribution is obtained. 


\subsubsection{Variable Efficiencies}

The real world usually does not provide experimenters with flat (constant) efficiencies. The measured quantity $x$ is defined to have efficiency $\epsilon(x)$. The efficiency $\epsilon(x)$ can be thought of as the relative probability of reconstructing the event at the measured value of $x$. Thus the total probability for the event is:

$$
\begin{aligned}
& P\left(x_{i}, n\right)=N(n) \epsilon\left(x_{i}\right) f\left(x_{i}, n\right) \\
& N(n)=\left(\int \epsilon(x) f(x, n) d x\right)^{-1}
\end{aligned}
$$

Once the probability function and its normalization have been determined the MaxLike procedure for determining the value of $n$ is the same as the case with uniform efficiencies. The probability distribution must be normalized and the efficiencies need to be well known for the entire range of $x$ even though the events might be clustered in a small range of $x$. With the introduction of variable efficiency the integration usually has to be done numerically.

\subsubsection{Resolution Functions}

The measured variable $x_{i}$ is measured with a known resolution, $R\left(x_{i}, x_{g}\right)$, where $x_{i}$ is the measured value and $x_{g}$ is the generated (true) value. $R\left(x_{i}, x_{g}\right)$ can have any functional shape (See Figure 50).

The measured quantity is $x_{i}$ and it is the generated value $x_{g}$ that is unknown, and 


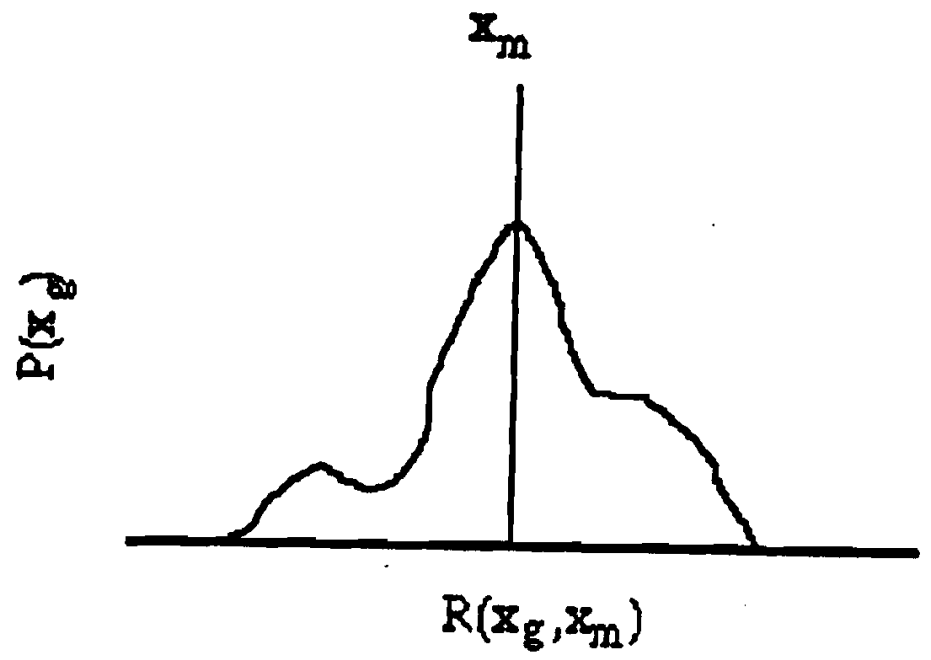

Figure 50: Generic resolution function $R\left(x_{i}, x_{g}\right)$ as function of $x_{q}$

$R\left(x_{i}, x_{g}\right)$ represents the probability that the generated value was $x_{0}$ given the measured value of $x_{i}$. The probability for the event to have a measured value of $x_{i}$ is:

$$
\begin{aligned}
& P\left(x_{i}, n\right)=\int N(n) \epsilon\left(x_{g}\right) f\left(x_{\theta}, n\right) R\left(x_{i}, x_{g}\right) d x_{\theta} \\
& N(n)=\left(\iint \epsilon\left(x_{\theta}\right) f\left(x_{\theta}, n\right) R\left(x, x_{g}\right) d x_{g} d x\right)^{-1}
\end{aligned}
$$

Note that the probability integral is over all generated values of $x_{0}$ whereas the normalization integral is over both $x_{g}$ and the measured variable $x_{i}$. This distinction is important since the measured variable $\left(x_{i}\right)$ may be linited to a small range but the generated variable $\left(x_{g}\right)$ can have all allowed values. The efficiencies must be determined for the full range of $x_{g}$. 


\subsubsection{Maxinum Likelihood Implementation in E653}

As described in the previous chapter the momentum estimation for the charm parent depends on the topology and visible characteristics of the decay. A decay dependent resolution function is also constructed for each decay. Thus the resolution function changes with each decay and forces the maximum likelihood normalization (Equation 50)to be calculated for each decay. Once the normalization has been determined the probability (eq. 49) for observing the decay is calculated and then this probability is combined with all the other probabilities in the event sample to form the maximum likelihood distribution (eq. 46).

All the elements to perforn the maximum likelihood calculation have been described except for the efficiencies. The following sections describe the efficiency distributions used in the fitting procedure and how they were obtained.

\subsection{Topological Efficiencies}

The E653 charm decay sample was divided into distinct topological categories. These decay topologies had different momentum resolution functions due to the differing number of missing neutrals in the topologies. The efficiencies for reconstructing individual decays are also different for the different topologies. This is clearly the case for the muonic decays, since the muon daughter had to pass special scanning selection criteria that the hadroni " daughters did not.

The efficiencies must also be determined as a function of the variable of interest. For 
single charm production studies the traditional kinematic variables are $X_{f}$ and $P_{t}^{2}$. These single charm efficiency distributions are then used to construct efficiency distributions for the charm pair.

\subsubsection{Single Charm Efficiencies}

The efficiencies for reconstructing charm decays were determined by using the GEANT Monte Carlo package to sinulate the spectrometer performance. Events were generated at the hit level and were processed through the complete spectrometer reconstruction code. The emulsion efficiencies were assumed to be flat for this analysis.

Charm mesons were produced in uncorrelated pairs and were allowed to decay via the known branching ratios. The charm mesons were produced using the differential cross section parameterization:

$$
\frac{d \sigma}{d X_{f} d P_{t}^{2}} \sim\left(1-X_{f}\right)^{n} \cdot e^{-b P_{t}^{2}}
$$

The user was allowed to define the $n$ and $b$ values used for charm generation. Correlated biases in the efficiencies were accounted for by requiring the $P_{t}^{2}$ distribution to reflect that seen in the data when determining the $X_{f}$ efficiencies (and vis versa for the $P_{t}^{2}$ efficiencies). Complete details of the single charm efficiencies can found elsewhere [35]. The $X_{f}$ efficiencies for muonic topologies in the enulsion are shown in Figure 51. and for hadronic decays in the emulsion $X_{f}$ efficiencies are shown in Figure 52. The efficiency for the hadronic decays - tend further into the the negative $X_{f}$ region than the muonic decays due to the selection cuts placed on the muonic tracks. The efficiency for all 
topologies peaks near the positive side of zero than falls rapidly zero in the negative $\mathrm{X}_{f}$ hemisphere. The loss of efficiency at negative $\mathrm{X}_{f}$ is due to the wide angle low momentum daughter tracks. The efficiencies also show a slight decline at $\mathrm{X}_{f}>0.5$. These fast forward particles will have the daughter tracks in the densely populated forward cone and will fail reconstruction due to pattern recognition failures in this region.

The $\mathrm{P}_{t}^{2}$ efficiencies for the muonic topologies in the main emulsion block are shown if Figure 53. The slight dip in the muonic efficiencies at small $P_{t}^{2}$ is due to the cliarm selection cuts placed on the $\mu$ track. The hadronic topologies are shown in Figure 54. The $P_{t}^{2}$ efficiencies are relatively flat, because there were no selectio cuts applied to these decays.

The precision of the efficiencies was limited by three colnerent sources: CPU hours, disk space and patience.

\subsection{Charm Pair Efficiencies}

Efficiencies for the charm pair distributions were determined by two different methods.

For the fitted kinematic quantities, $\mathrm{X}_{f}$ and $\mathrm{P}_{t}^{2}$, the efficiencies were calculated on an event by event basis, integrating the individual single charm efficiencies to obtain the overall efficiency for the charm pair.

For the pair $\boldsymbol{X}_{\boldsymbol{f}}$ efficiencies the integration was as follows:

$$
\epsilon\left(X_{f P A I R}\right)=\int_{-1}^{1} \int_{-1}^{1} N \cdot \epsilon\left(X_{f}(D)\right) \cdot \epsilon\left(X_{f}(\bar{D})\right) \cdot\left(1-X_{f}(D)\right)^{n} \cdot\left(1-X_{f}(D)\right)^{n} d X_{f}(D) d X_{f}(\bar{D})
$$



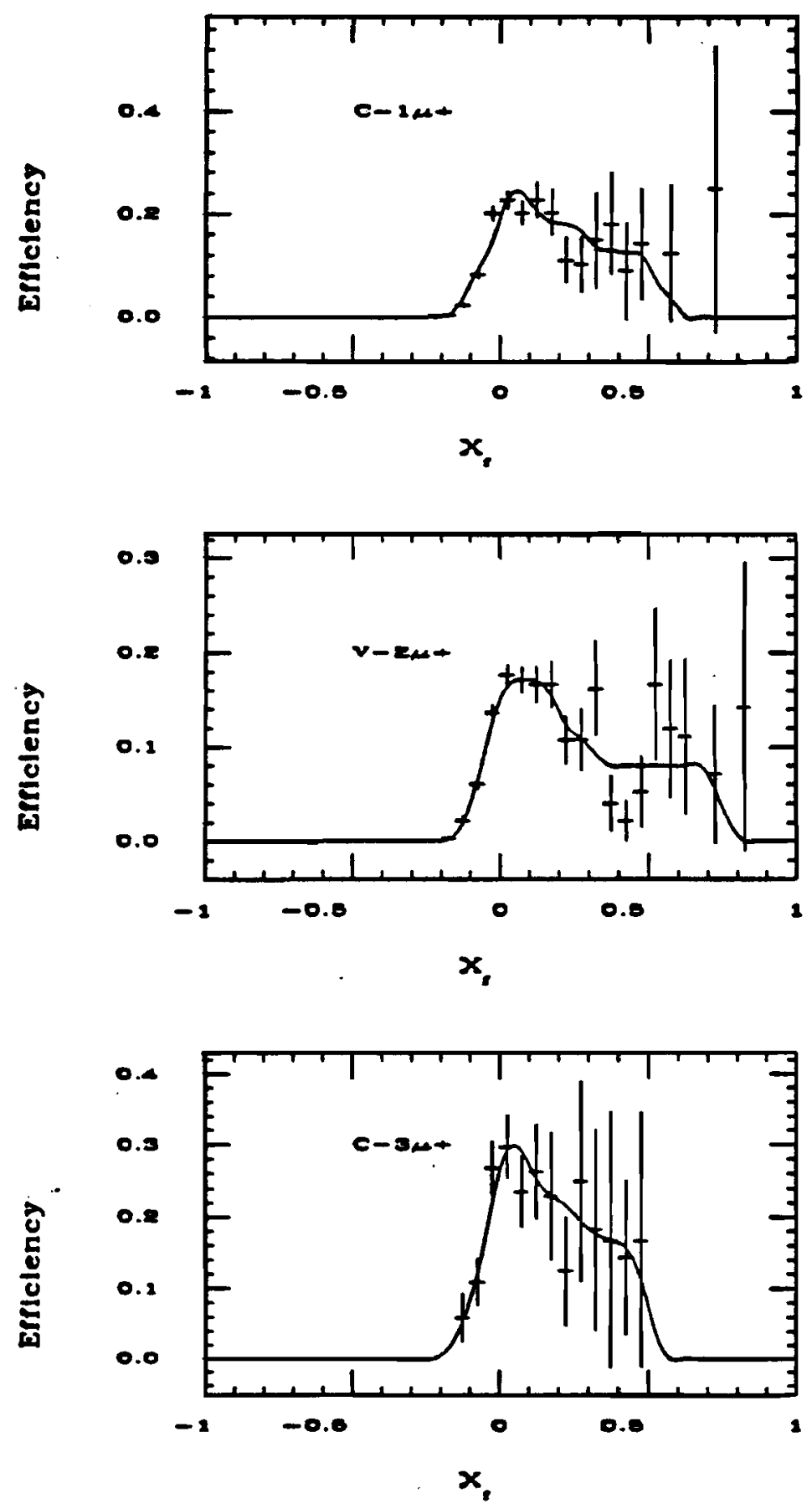

Figure 51: $X_{f}$ efficiencies for muonic decays in the emulsion block. 

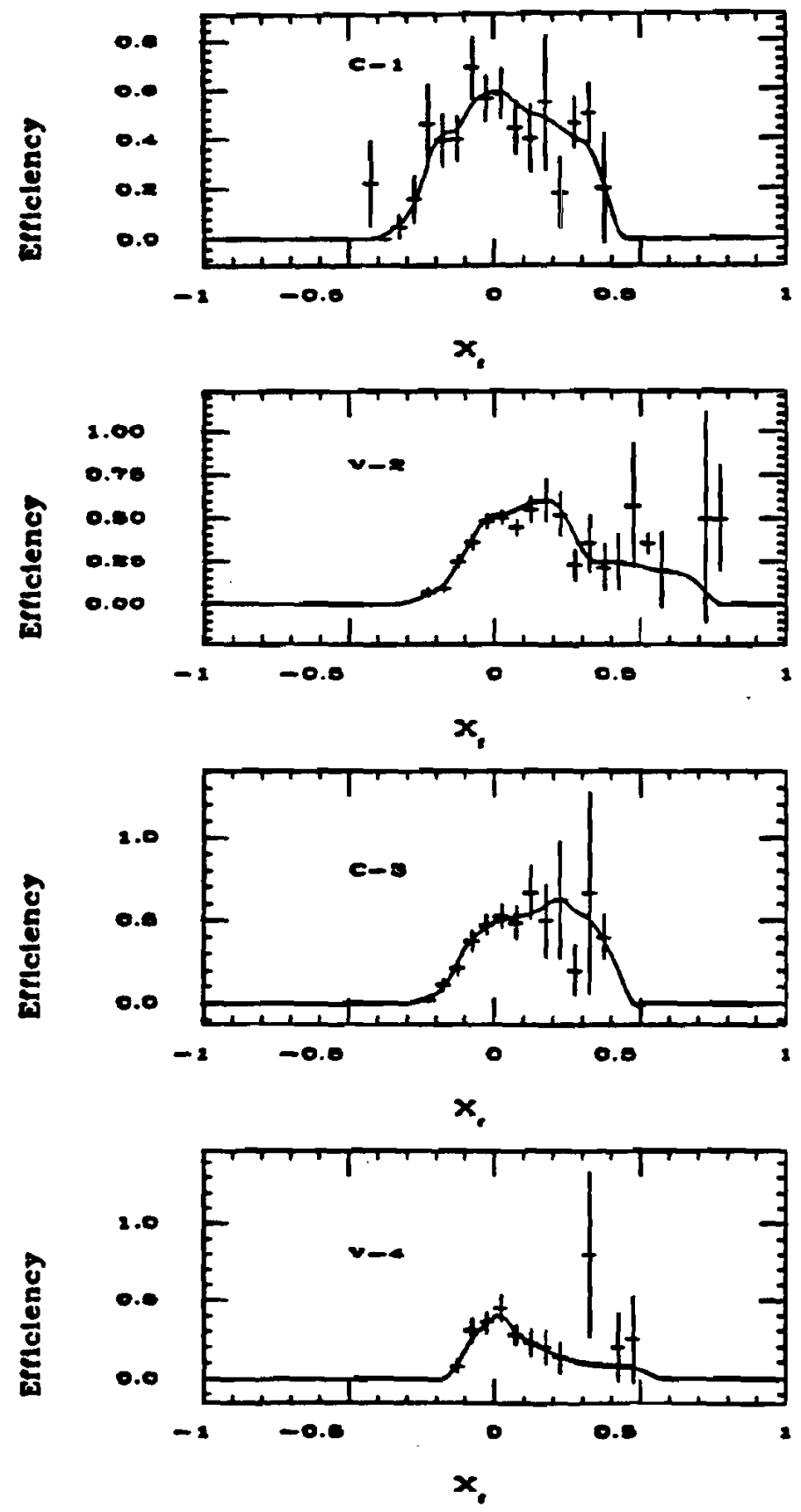

Figure 52: $\boldsymbol{X}_{f}$ efficiencies for hadronic decays in the emulsion block. 

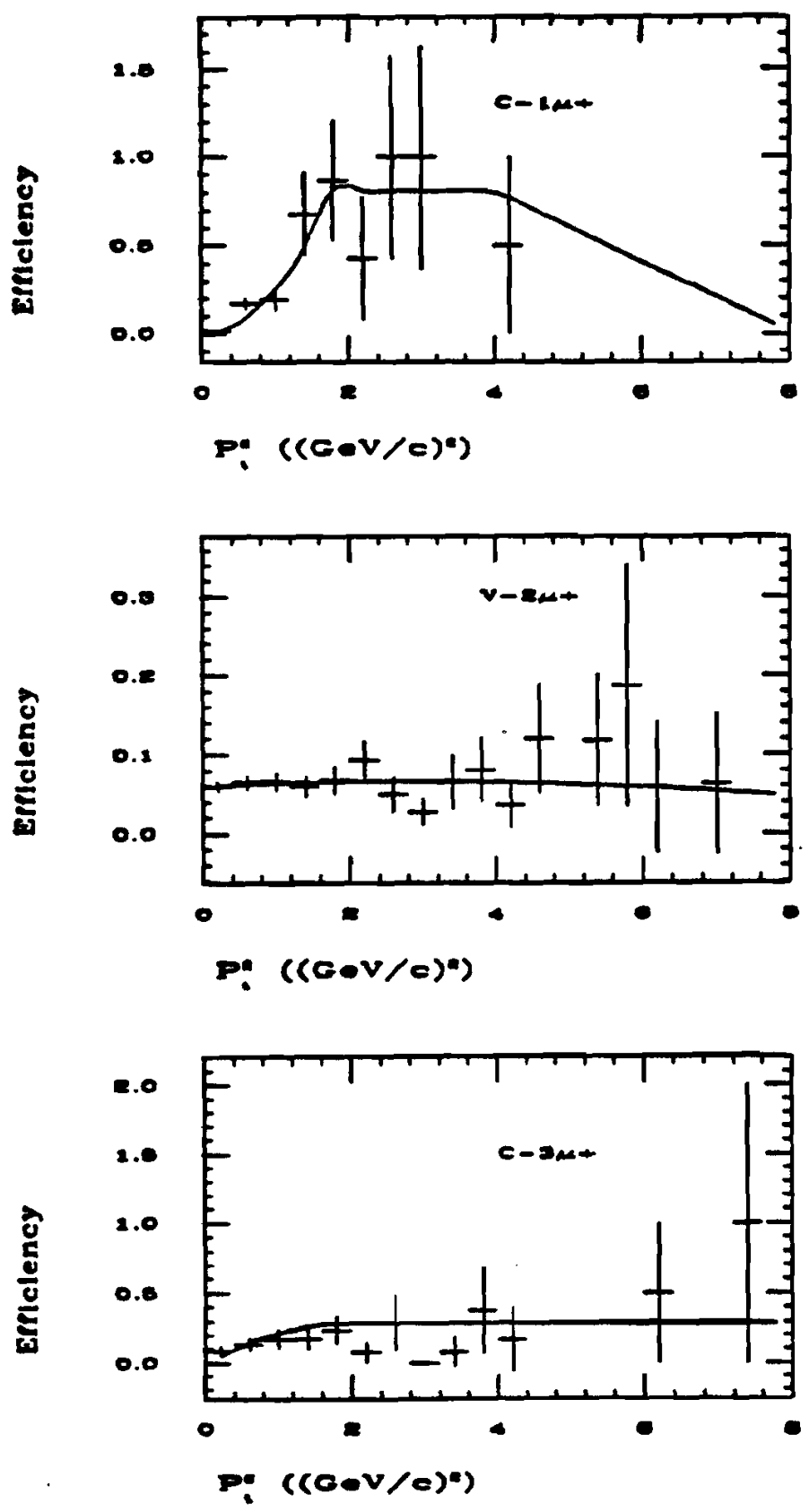

Figure 53: $P_{t}^{2}$ efficiencies for muonic decays in the enulsion block. 

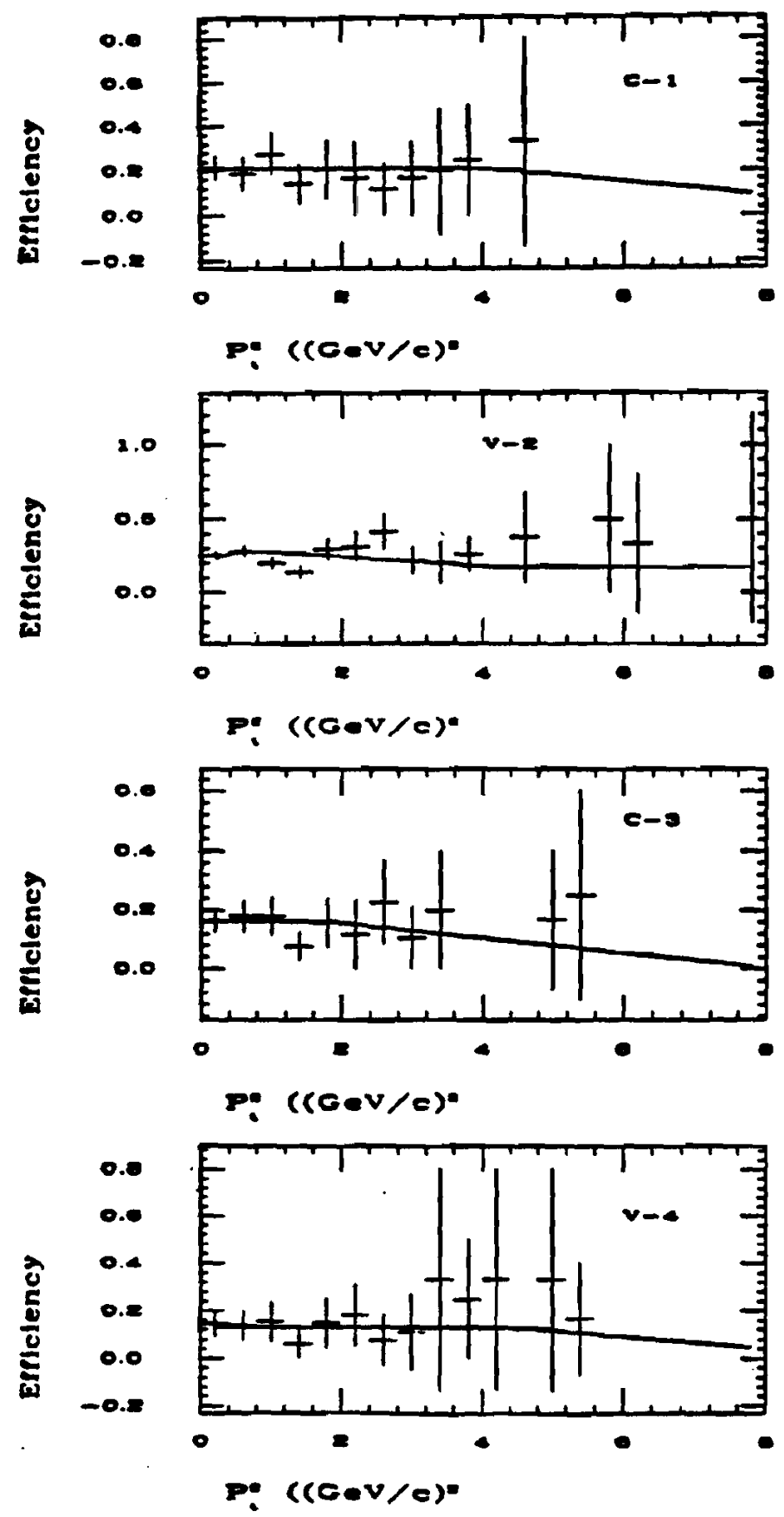

Figure 54: $P_{t}^{2}$ efficiencies for hachrouic decays in the emulsion block. 


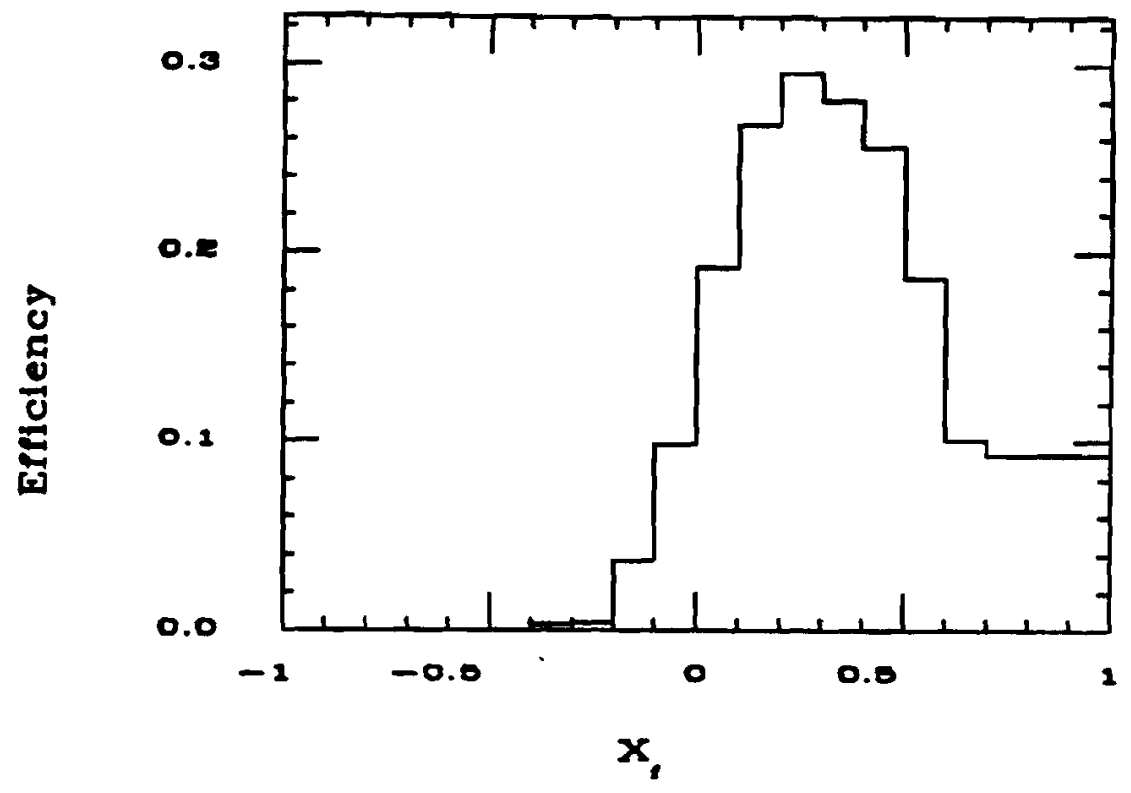

Figure 55: Average efficiency as a function of $X_{f}$ for the 37 charm pairs.

with:

$$
N^{-1}=\int_{-1}^{1} \int_{-1}^{1}\left(1-X_{f}(D)\right)^{n} \cdot\left(1-X_{f}(\tilde{D})\right)^{n} d X_{f}(D) d X_{f}(\tilde{D})
$$

and:

$$
X_{f P A I R}=f\left(X_{f}(D), X_{f}(\bar{D})\right)
$$

The $n$ value was set to the measured $\mathbf{n}$ from the single charm production studies [35]. The average efficiency distribution, weighted by charm pair topology, for all 37 charm pairs is shown in Figure 55. The charm pair $\mathrm{X}_{f}$ efficiency distribution is similar to that seen in the single charm distribution. The loss of efficiency for large $\mathrm{X}_{f}$ charm pairs is due to the daughter tracks occupying the densely populated forward cone of the event.

The same approach was used for the efficiency as a function of $P_{t}^{2}$. 


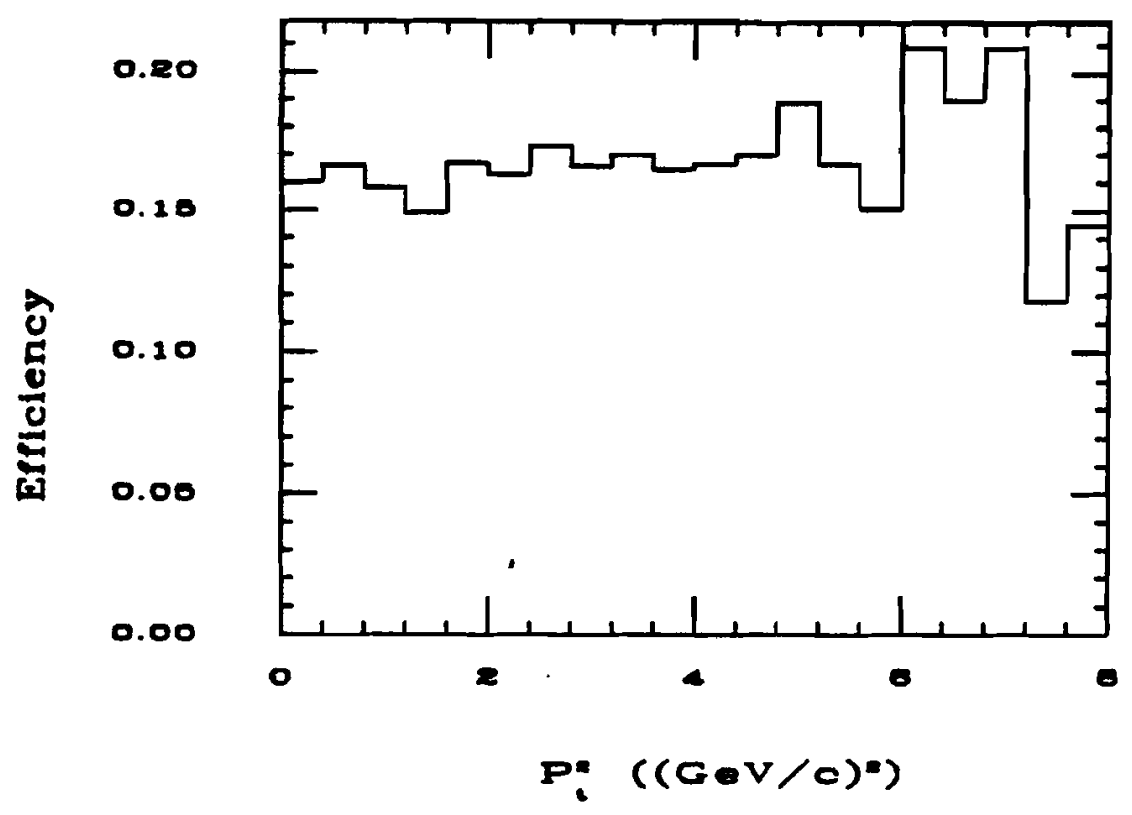

Figure 56: Average efficiency as a function of $P_{t}^{2}$ for the 37 charm pairs.

$$
\epsilon\left(P_{t P A I R}^{2}\right)=\int_{0}^{P_{t \text { mee }}^{2}} \int_{0}^{P_{t \text { mes }}^{2}} N \cdot \epsilon\left(P_{t}^{2}(D)\right) \cdot \epsilon\left(P_{t}^{2}(D)\right) \cdot e^{-b P_{t}^{2}(D)} \cdot e^{-b P_{t}^{2}(D)} d P_{t}^{2}(D) d P_{t}^{2}(D)
$$

with:

$$
N=\int_{0}^{P_{\text {tmese }}^{2}} \int_{0}^{P_{t \text { max }}^{2}} e^{-b P_{t}^{2}(D)} \cdot e^{-6 P_{t}^{2}(\bar{D})} d P_{t}^{2}(D) d P_{t}^{2}(\bar{D})
$$

and:

$$
P_{t P A I R}^{2}=f\left(P_{t}^{2}(D), P_{t}^{2}(D)\right)
$$

The value of $b$ in the exponential was set to $1.0(\mathrm{GeV} / \mathrm{c})^{-2}$ measured in the single charm distributions. The average efficiency as a function of $P_{t}^{2}$ is shown in Figure 56. The charn pair $P_{t}^{2}$ efficiency was flat for $P_{t}^{2}<(6.0 \mathrm{GeV} / \mathrm{c})^{2}$.

For quantities that were exclusively charm pair distributions the efficiencies were 
determined via Monte Carlo generated charm pairs. Individual charm mesons were generated with the appropriate differential cross section distribution. The production distributions were then trimmed with the known single charm reconstruction efficiencies in $X_{f}$ and $P_{t}^{2}$. These uncorrelated accepted mesons were than used to form charm pair events. The flat $\Phi_{t}$ distribution of these uncorrelated pairs was trim to reproduce the $\Phi_{t}$ distribution seen in our data. Figure 57 shows the efficiency as a function of charm pair mass, averaged to reflect the topologies represented in the data. The same uncorrelated MC was used to obtain the rapidity gap efficiencies shown in Figure 58. The rapidity gap and charm pair mass efficiency distributions look simular because these two quantities are obtain from the same variables, $\mathrm{X}_{f}$ and $\mathrm{P}_{t}^{2}$ of the individual charm. The efficiencies fall at large mass and rapidity gap because these events require a large difference in the individual $\mathrm{X}_{f}$ values and the magnitude of this gap $\left(\mathrm{X}_{f G A P}\right)$ is restricted by the individual charm $\mathrm{X}_{f}$ efficiency and production distribution. ${ }^{1}$

\subsection{Validity of the Maximum Likelihood Technique}

With the efficiencies understood the full maximum likelihood technique must be tested with Monte Carlo events to study any biases from the momentum estimator or efficiencies. The single charn studies are described in detail elsewhere [35]. Nichols compares the generated Monte Carlo value to the value determined via the maximum likelihood tecluique for the $X_{f}$ and $P_{t}^{2}$ single charm differential cross section distributions. T ${ }^{1}$ e

\footnotetext{
${ }^{1}$ The $\mathrm{X}_{f G A P}$ is defined as $\mathrm{X}_{\text {fGAP }}(D D)=\left|\mathrm{X}_{f}(D)-\mathrm{X}_{f}(D)\right|$. The charm pair mass and rapidity gap are strongly correlated to the $\mathrm{X}_{f G A P}$ and the efficiency distribution of the $\mathrm{X}_{f O A P}$ decreases at large $\mathrm{X}_{f O A P}$.
} 


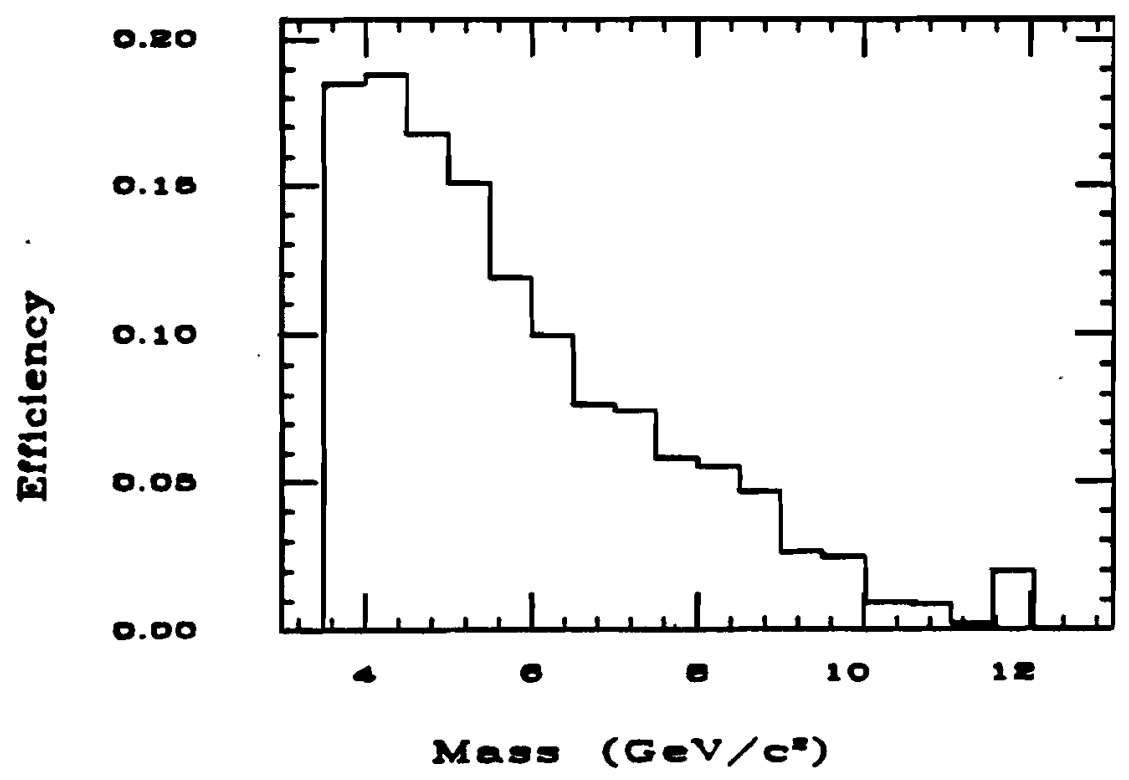

Figure 57: Average efficiency distribution as a function of charm pair mass.

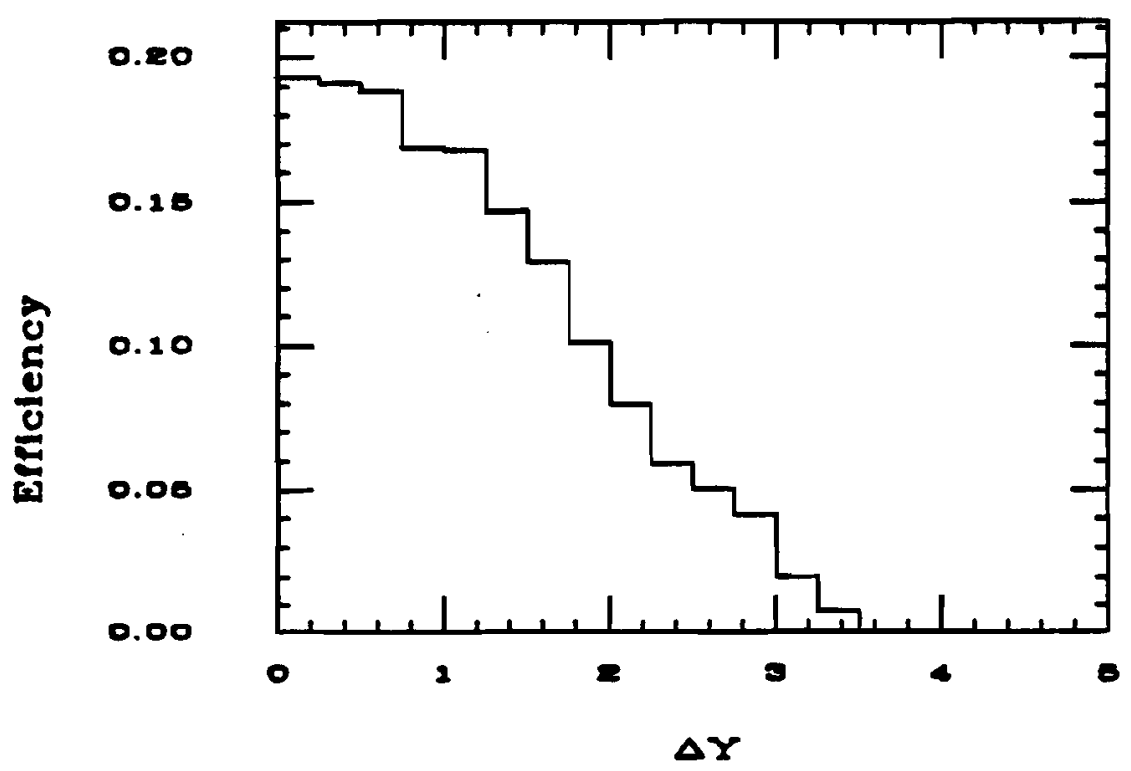

Figure 58: Average efficiency distribution as a function of charm pair rapidity gap. 
comparison is quite good for the region around the values measured in the data $n=10.0$ and $b=1.0(\mathrm{GeV} / \mathrm{c})^{-2}$.

For the charm pair distributions the test is not as simple as comparing the input parameter to the output value since charm pairs are generated by combining individual charm mesons produced with a known production distribution. The generated production distribution for the charm pairs must also be determined by $\chi^{2}$ fitting the generated distribution. The vector addition of the individual charm momentum, generated with an exponential distribution in $\mathrm{P}_{t}^{2}$, does not return an exponential distribution for the pair $\mathrm{P}_{t}^{2}$. It was observed that the introduction of the $\Phi_{t}$ distribution caused the $P_{t}^{2}$ distribution to become more non-exponential. For purposes of this test the correlated $\Phi_{t}$ distribution was removed to allow testing of the maximum likelihood technique on the charm pair distributions. The generated pair $P_{t}^{2}$ distribution with a flat $\Phi_{t}$ distribution did not return a good fit to a single exponential and this is refelected in the plot. The fact that the Monte Carlo single charm $\mathbf{P}_{t}^{2}$ distributions return acceptable fits gives one confidence that the method works and can be trusted for the cliarm pair distributions. Figures 59 and 60 compare the generated Monte Carlo production paraneters with the values returned by the maximum likelihood method. The fact that the fit for pairs generated with a flat $\mathrm{X}_{f}$ distribution did not return the proper $n$ value can be attributed to the fact that the efficiencies at large $\mathrm{X}_{f}$ are not very well known. 


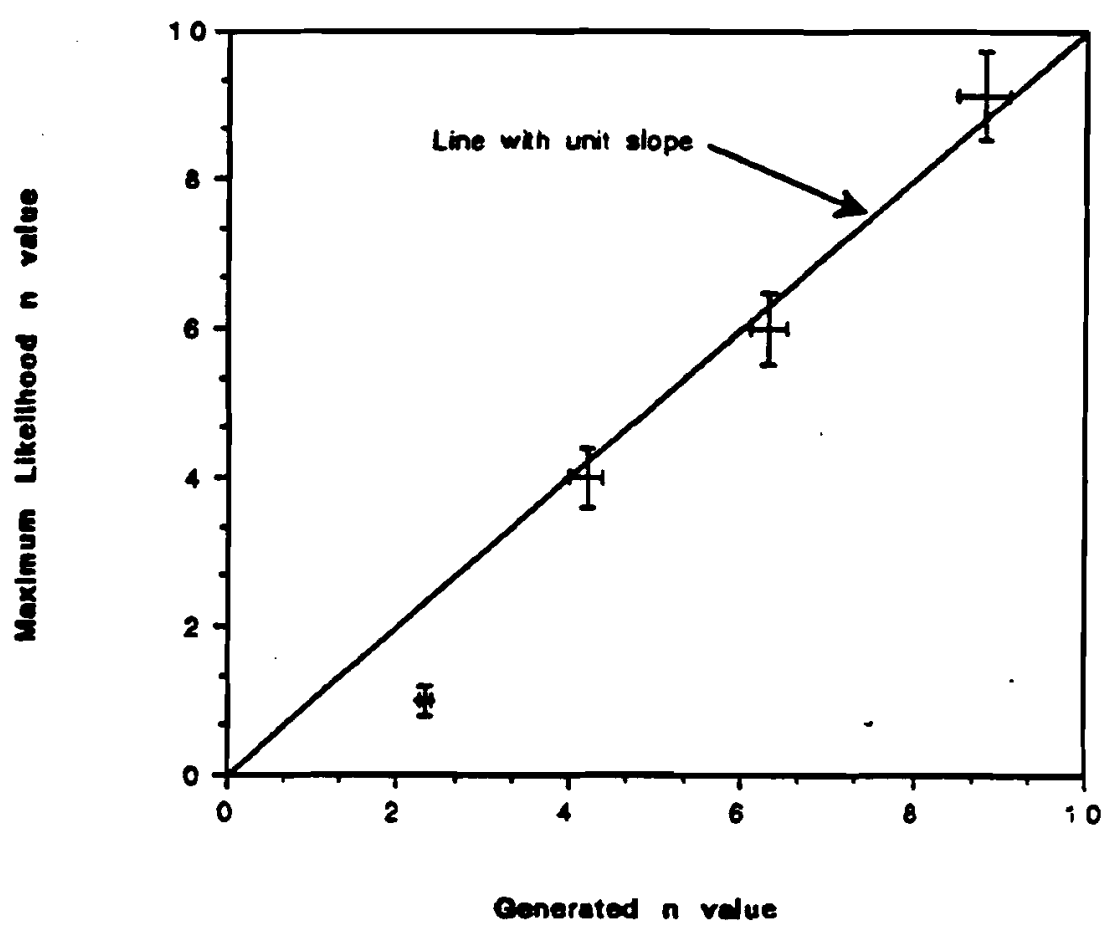

Figure 59: Comparison of the input Monte Carlo n value with that retumed by the maximum likelihood method for chann pairs.

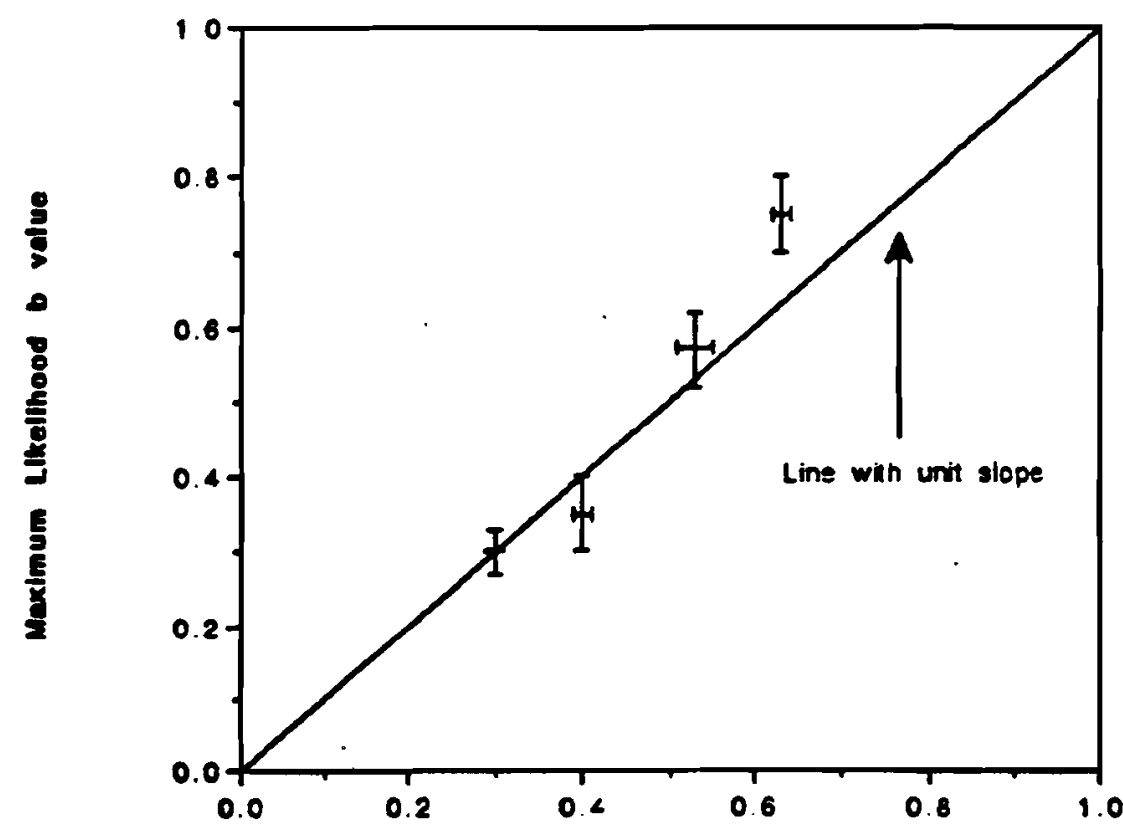

Cenerated velue

Figure in: Comparison of the input Monte Carlo b value with that returned by the maximun likelihood method for charm pairs. 


\section{Chapter 8}

\section{Charm Pair Production and}

\section{Correlation Results}

\subsection{Introduction}

The study of charm pair production characteristics allows one to measure the kinematic quantities involved in charm production and to study production correlations within the charm pair system. The kinematic quantities of the charm pair reflect the kinematic distribution of the parton (gluon) momentum responsible for charm production. Previous experiments (LEBC- $\pi$ P had 12 charm pairs and LEBC-PP had 17 pairs) were strongly linited by statistics and presented weighted distributions and their averages. The 37 charm pair events in this sample permit fits to be performed to the $\mathrm{X}_{f}$ and $\mathrm{P}_{t}^{2}$ distributions of the charm pair system for the first time. In addition to fitting the differential pair cross section in $\mathrm{X}_{f}, \mathrm{P}_{t}^{2}$ and pair mass, the kinematic distributions are 
compared to $\mathrm{MC}$ produced charm pairs. The averages for the measured quantities in the data are compared to those measured previously and with averages returned from the Monte Carlo generated charm pairs. A complete listing of the 37 charm pair events and the associated kinematic quantities can be found in Appendix B.

\subsection{Charm Pair Monte Carlo}

The data are compared to charm pairs produced by two different Monte Carlos, the LUND MC and a $\Phi_{t}$ correlated Monte Carlo developed by the author. The LUND MC generates charm pairs via the leading order production diagrams and is described in more detailed in Appendix A. The LUND MC uses only the leading order diagrams in the production process which account for less than a half of the cross section $[9,12]$. There are a number of user definable parameters in the LUND MC that allow the user to mold the charm pair distributions. With the large number of adjustable parameters and using only leading order production diagrams it is impossible to determine if agreement or disagreemant between the LUND MC and the data is just a fortuitous combination of input parameters or real physics. The LUND MC was used exhaustively by the LEBC collaboration in their cham pair analysis and the comparison of LUND MC to this data is done for completeness.

In addition to the LUND MC, the data is compared to an uncorrelated pair Monte Carlo. This Monte Carlo consisted of single charm particles generated using the measured differential production distributions in $\mathrm{X}_{f}$ and $\mathrm{P}_{t}^{2}$ [35]. The two single charm particles 
were then combined to form charm pairs. The azimuthal opening angle distribution, $\Phi_{t}$, for these uncorrelated charm pairs was flat. The $\Phi_{t}$ distribution seen in our data and in the LEBC data was peaked at $180^{\circ}$. This $\Phi_{t}$ correlation was installed in the Monte Carlo, by "trimming" the $\Phi_{t}$ of the uncorrelated charm pairs. This "trimming" forced the charm pairs generated by this Monte Carlo to have the same $\Phi_{t}$ distribution that was observed in our data. This Monte Carlo will be referred to as the $\Phi_{t}$ correlated Monte Carlo throughout this section. It is important to emphasize that the correlation in $\Phi_{t}$ is the only correlation in the charm pair system installed in this Monte Carlo.

\subsection{Pair $\mathrm{X}_{f}$ Distribution}

Figure 61 shows the weighted $\mathrm{X}_{f}$ distribution for the charm pairs. The maximum likelihood fit to $\left(1-X_{f}\right)^{n}$ yields $n=5.0 \pm 1.5$, and this curve is represented by the solid line. Charm pairs generated with the $\Phi_{t}$ correlated $\mathrm{MC}$ yield the $\mathrm{X}_{f}$ distribution represented by the dashed histogram. A fit to the $\Phi_{t}$ correlated $\mathrm{MC} \mathrm{X}_{f}$ distribution yields $n=6.0 \pm 1.0$. The pair $\mathrm{X}_{f}$ distribution is indistinguishable from the distribution obtained from $\Phi_{t}$ correlated $M C$ charm pairs. The $\mathrm{X}_{f}$ distribution for the charm pairs is uncorrelated at this level of statistics. The $\mathrm{X}_{f}$ distribution is also consistent with being symmetric about $\mathrm{X}_{f}=0$. 


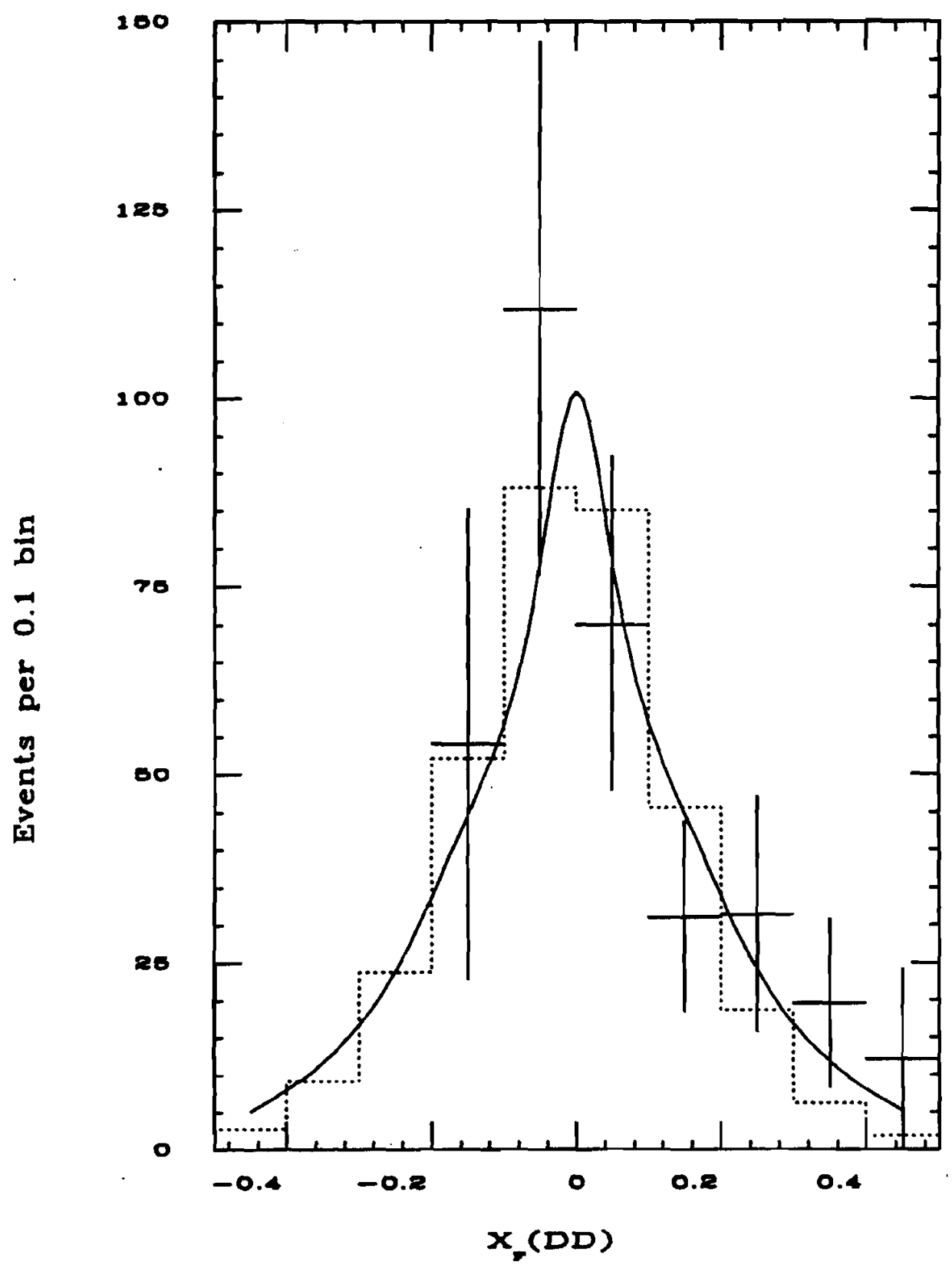

Figure 61: The $\mathrm{X}_{f}$ distribution for the 37 charm pairs. The solid line is the result of a maximum likeliliood lit to $\left(1-\mathrm{X}_{f}\right)^{n}$. The dashed histogram is from $M C \Phi_{t}$ correlated charm pairs. 


\subsection{Pair $\mathbf{P}_{t}^{2}$ Distribution}

The $P_{t}^{2}$ distribution for the charm pair was assumed to be represented by $e^{-6 P_{t}^{2}}$ even though MC studies showed that correlations in the $\Phi_{t}$ distribution will affect the $P_{t}^{2}$ parameterization. Monte Carlo chann pairs generated uncorrelated kinematically but with the $\Phi_{t}$ angular correlation did not yield a pair $P_{t}^{2}$ distribution that could be represented by $e^{-b P_{t}^{2}}$. Figure 62 shows the weighted $P_{t}^{2}$ distribution for the 37 pairs and the solid line is the result of the fit which yielded $b=0.65_{-0.08}^{+0.10}(G e V / c)^{-2}$. The dashed histogram is the $\mathrm{P}_{t}^{2}$ distribution for $\mathrm{MC} \Phi_{t}$ correlated charm pairs. The $\mathrm{MC} \mathrm{P}_{t}^{2}$ distribution does not agree with the data $P_{t}^{2}$ distribution at low and high $P_{t}^{2}$. The $P_{t}^{2}$ distribution shows evidence for a tail at large $P_{t}^{2}$. This tail could be from higher order charm production diagrams like $g g \rightarrow g q \bar{q}$ where the charm pair is produced with larger $\mathrm{P}_{t}^{2}$ than the leading order $2 \rightarrow 2$ diagrams. A single exponential parameterization of the $P_{t}^{2}$ distribution is not valid for charm pairs with large $\mathrm{P}_{t}^{2}$.

The average of the efficiency corrected $P_{t}^{2}$ distribution is $2.32 \pm 0.36(\mathrm{GeV} / \mathrm{c})^{2}$. Figure 63 shows the $\left\langle\mathrm{P}_{t}^{2}\right\rangle$ versus $s$ for this experiment and the LEBC-PP [51] measurement obtained at a lower value of s. In Drell-Yan di-lepton production the average $P_{t}^{2}$ is expected to rise linearly with $s$. The intercept at $s=0$ yields the average intrinsic $k_{t}^{2}$ of the partons involved in the process. Di-lepton studies measured the $\mathbf{k}_{t}^{2}$ of quarks and similar measurements in charm pair analysis will measure the $\mathbf{k}_{t}^{2}$ of the gluons inside the incident and target particle. Using the $t=0$ measured points the intrinsic $k_{t}^{2}$ of the gluons involved in charm production is $0.70 \pm 0.70(\mathrm{GeV} / \mathrm{c})^{2}$. This value is consistent with the value 


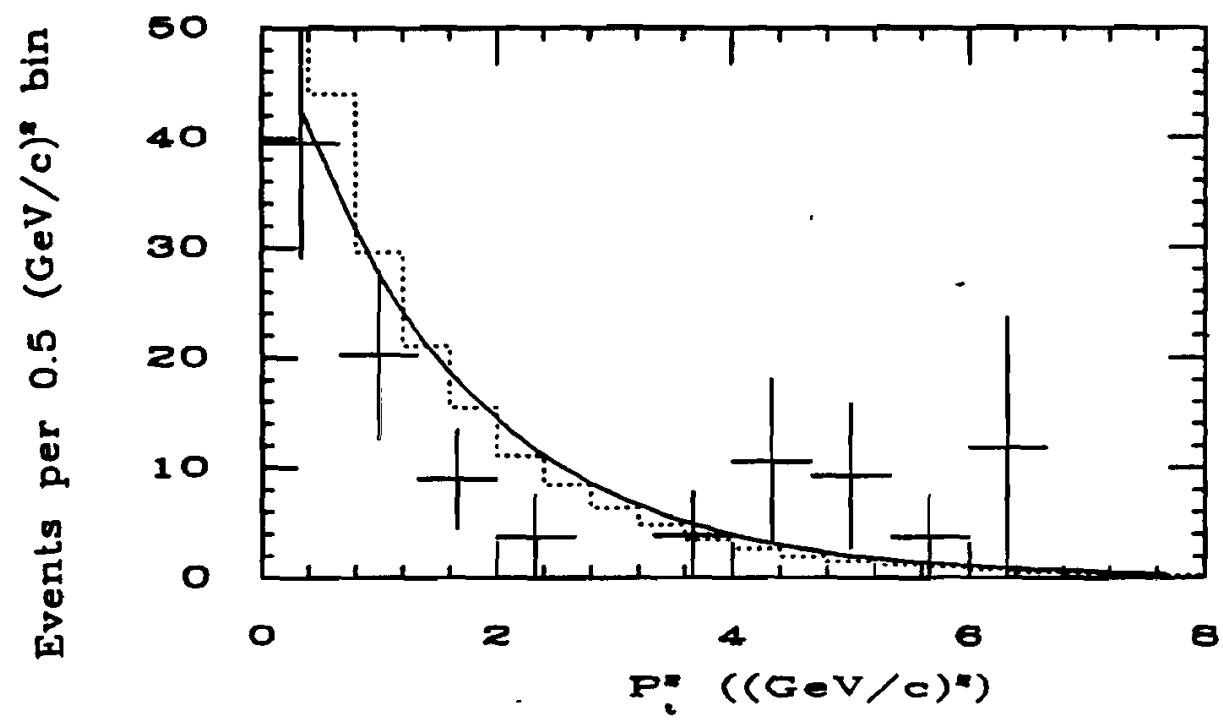

Figure 62: The weighted $P_{t}^{2}$ distribution for the charm pairs. The solid line is the result of a maximum likelihood fit to $e^{-b P_{t}^{2}}$. The dashed histogram is for $\Phi_{t}$ correlated cliarm pairs.

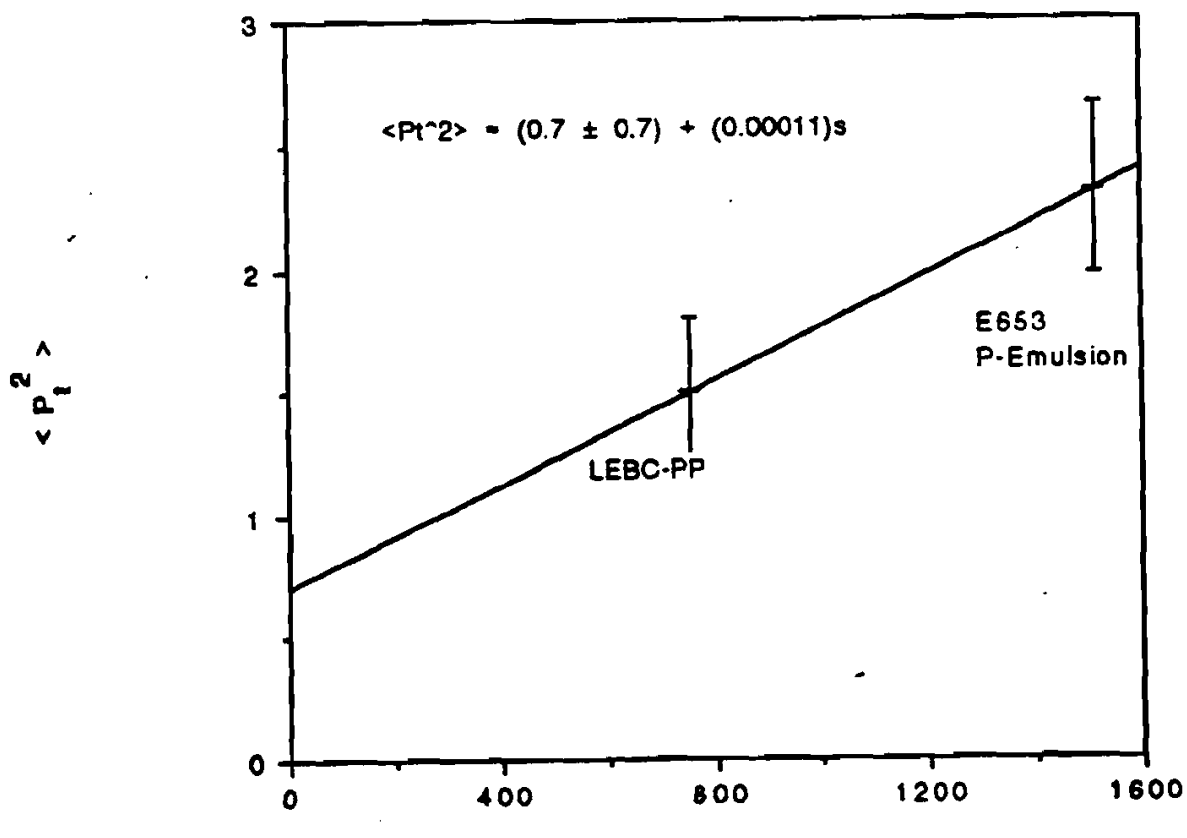

Figure 63: The $\left\langle\mathrm{P}_{t}^{2}\right\rangle$ versus 3 for E653 and LEBC-PP charm pairs. The curve is a least squares fit to $\left\langle\mathrm{P}_{t}^{2}\right\rangle=\mathrm{k}_{t}^{2}+\mathrm{s} \cdot$ constant. 
measured in Drell-Yan di-lepton production [21].

\subsection{Pair Azimuthal Opening Angle $\left(\Phi_{t}\right)$}

The azimuthal opening angle $\left(\Phi_{t}\right)$ of the charm pair is shown in Figure 64 . The $\Phi_{t}$ distribution peaks at $180^{\circ}$ - reflecting a back-to-back nature in charm production. The average of the distribution is $113.2^{\circ} \pm 9.0^{\circ}$. This value is consistent with those measured previously by LEBC (See Table 24).

\subsection{Charm Pair Mass}

The mass of the charm pair reflects the " $\mathrm{q}^{2}$ " of the production process. The weighted mass distribution is shown in Figure 65. The dashed histogram is for $\Phi_{t}$ correlated charm pairs. The solid line is the result of a maximum likelihood fit to $e^{-a M(D D)}$ without taking into account the mass resolution function. The fit yielded $a=0.75 \pm 0.15\left(\mathrm{GeV} / \mathrm{c}^{2}\right)^{-1}$. The parameterzation $e^{-\alpha M}$ was motivated by Drell-Yan di-lepton production. Di-lepton mass plots were done at constant di-lepton rapidity, due to the lack of statistics and relatively small range in rapidity for the charm pairs, all 37 charm pair events in this sample were used in the fit. By converting the exponential to $e^{-c \sqrt{\tau}}$ values for c obtained at different COM energies can be compared. It will be interesting to see how well this mass scaling holds between experiments and for large $\sqrt{\tau}$ or $\mathrm{M}(\mathrm{D} \bar{D})$.

The average of the weighted distribution is $4.94 \pm 0.17 \mathrm{Gev} / \mathrm{c}^{2}$ which is slightly higher than for previous measurements. E653 with a higher COM energy has probed a higher 


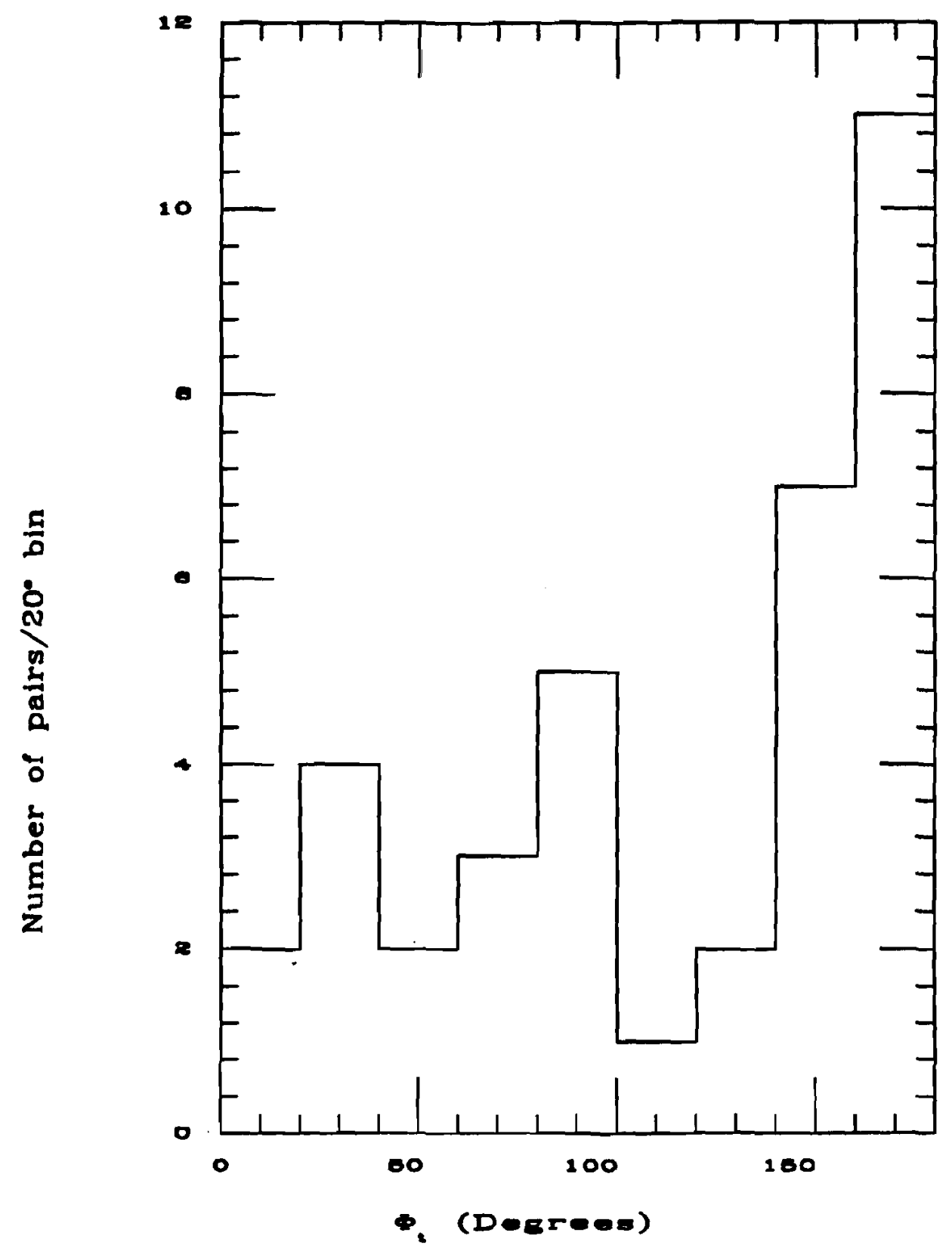

Figure 64: $\Phi_{t}$; Azimuthal opening angle of the charm pair) distribution isr the 37 charm pairs. 


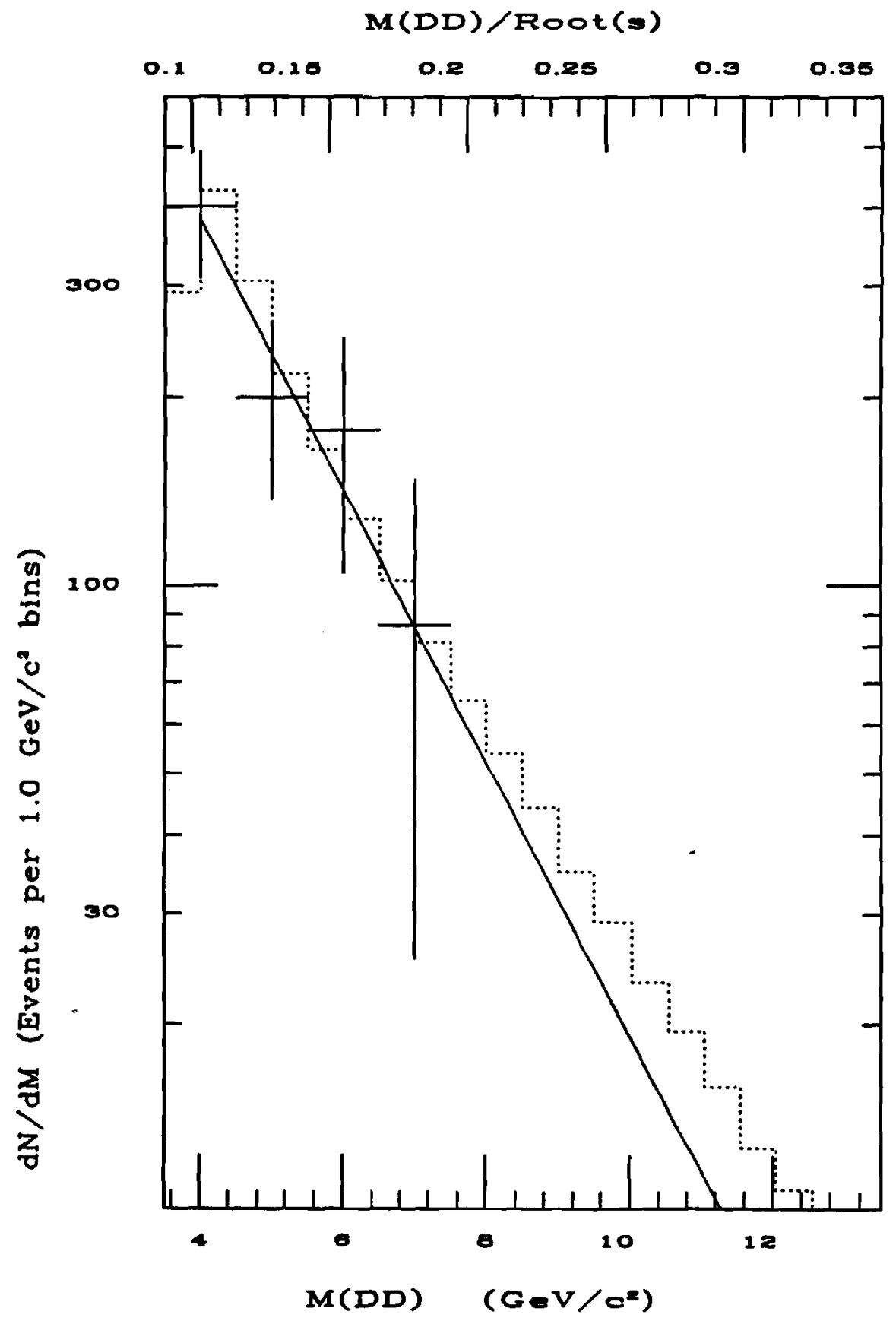

Figure 65: Charm pair mass distribution. The solid line is the result of a fit to $e^{-a M}$. The dotted listogram is the mass distribution obtained from the $\Phi_{t}$ corrrlal ed MC. 
mass range than the $L E B C$ data.

\subsection{Charm Pair Rapidity Gap $(\Delta Y)$}

The weighted rapidity gap distribution is shown in Figure 66. Monte Carlo $\Phi_{t}$ correlated charm pairs do not reproduce the $\Delta \mathrm{Y}$ distribution. The LEBC- $\pi \mathrm{P}$ charm pair $\Delta \mathrm{Y}$ distribution was slightly wider than that obtained from uncorrelated charm pairs [52]. The $\Phi_{t}$ correlated charm pairs have some momentum balance in the plane transverse to the beam direction due to the $\Phi_{t}$ correlation installed in the MC. Momentum balancing along the beam direction is not taken into account. By balancing momentum along the beam direction the rapidity gap would by broadened. Berger has pointed out that the shape of the rapidity gap depends distribution on whether the production was through $q \bar{q}$ anniluilation or $g g$ fusion, but performed the calculations only for beauty quarks [12].

\subsection{Comparison to previous results}

Comparison of the LEBC $\pi-P$ and P-P charm pair production results with these results is shown in Table 24 [51,52]. Table 24 lists the weighted averages for the pair kinematic variables $\left(\mathrm{X}_{f}, \mathrm{P}_{t}, \Phi_{t}\right.$, mass and $\left.\Delta \mathrm{Y}\right)$.

The averages of the $\Phi_{t}$ distributions for the three experiments agree quite well. All three averages are lower than the average returned by the Lund MC.

The average rapidity gap measured at $\sqrt{s}=38.8 \mathrm{GeV}$ has increased slightly compared to the LEBC-PP result at $\sqrt{s}=27.4 \mathrm{GeV}$. The measurements agree within statistics. The 


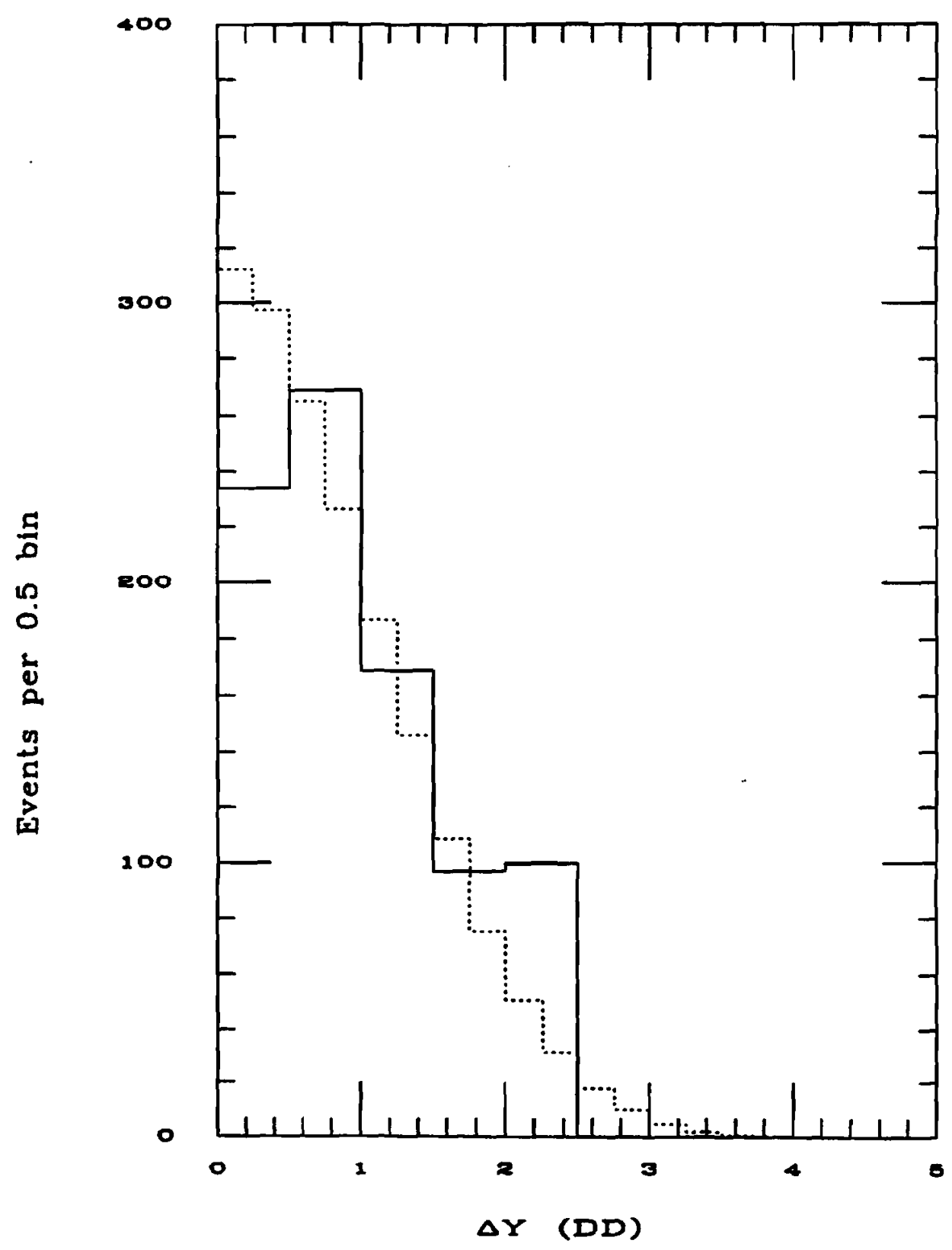

Figure 66: The rapidity gap distribution of the charm pairs. The solicl listogram is for the data. The dashed histogram is for $\Phi_{t}$ correlated MC charm pairs. 


\begin{tabular}{|c|c|c|c|c|c|c|c|}
\hline Experiment & $\begin{array}{l}\sqrt{s} \\
\mathrm{GeV}\end{array}$ & $\begin{array}{l}\text { Number } \\
\text { of Events }\end{array}$ & $\begin{array}{l}<M(D D)> \\
\mathrm{GeV} / \mathrm{c}^{2}\end{array}$ & $\begin{array}{l}\left\langle X_{f}(D D)\right\rangle \\
\left(X_{f}>0\right)\end{array}$ & $\begin{array}{l}\left\langle P_{l}^{2}(D D)>\right. \\
(\mathrm{GeV} / \mathrm{c})^{2}\end{array}$ & $<\Delta Y\rangle$ & $\begin{array}{l}\left\langle\Phi_{t}>\right. \\
\text { Degrees }\end{array}$ \\
\hline E653 & 38.8 & 37 & $4.94 \pm 0.17$ & $0.172 \pm 0.022$ & $2.32 \pm 0.36$ & $1.00 \pm 0.11$ & $113.2 \pm 9.0$ \\
\hline $\begin{array}{l}\text { P-Emulsion } \\
\Phi_{t} \\
\text { Correlated MC }\end{array}$ & & & 4.71 & 0.13 & 1.52 & 0.87 & 113.5 \\
\hline LUND & & & 5.67 & 0.18 & 1.17 & 1.37 & 121 \\
\hline LEBC-PP & 27.4 & 17 & $4.65 \pm 0.13$ & $0.18 \pm 0.03$ & $1.50 \pm 0.30$ & $1.02 \pm 0.12$ & $\begin{array}{l}105 \pm 5 \\
105 \text { events }\end{array}$ \\
\hline LUND & & & 4.82 & 0.19 & 0.88 & 0.98 & 119 \\
\hline$\overline{\mathrm{LEBC}}-\pi \mathrm{P}$ & 26.0 & 12 & $4.50 \pm 0.16$ & $0.25 \pm 0.07$ & $1.65 \pm 0.40$ & $0.80 \pm 0.14$ & $\begin{array}{l}115 \pm 8 \\
57 \text { events }\end{array}$ \\
\hline LUND & & & 4.60 & 0.25 & 0.75 & 0.77 & 126 \\
\hline
\end{tabular}

Table 24: Comparison of weighted average values measured in two previous charm pair experiments and the values presented in this thesis.

$\left\langle\mathrm{P}_{t}^{2}>\right.$ reported here is larger than for the LEBC-PP measurement and this can be attributed to a Drell-Yan like scaling of the average $\mathrm{P}_{t}^{2}$ with s. The $\left\langle\mathrm{P}_{t}^{2}\right\rangle$ value returned from the LUND MC does not agree with any of the charm pair measurements.

The average $\left\langle\mathrm{X}_{f}>\right.$ measured at $\sqrt{s}=38.8 \mathrm{GeV}$ and $27.4 \mathrm{GeV}$ agree remarkably well. This is especially surprising since the single charm production results from E653 and LEBC-PP do not agree $[35,50]$.

The $\langle M\rangle$ for the charm pairs is expected to rise with larger values of $\sqrt{s}$. This rise is seen when comparing the $\langle M\rangle$ measured by the three charm pair experiments. Charm production at high $<M>$ or high $q^{2}$ can be more easily treated theoretically by using perturbative methods. A high statistics charm pair experiment would allow the study of chann production in the purely perturbative region (high charm pair mass) and connect to the non-perturbative region (low charm pair mass). E653 has insufficient statistics to do this analysis, however, the increase in $\langle M\rangle$ gives one hope that such an experiment 
CHAPTER 8. CHARM PAIR PRODUCTION AND CORRELATION RESULTS 148

is possible (E653-RUNII). 


\section{Chapter 9}

\section{Conclusions}

A complete analysis of hadrouic production of 37 charm pairs has been presented. The differential cross section for the charm pairs was fit to the following empirical formula:

$$
\frac{d \sigma}{d X_{f} d P_{t}^{2}} \sim\left(1-\left|X_{f}\right|\right)^{n} e^{-6 P_{t}^{2}}
$$

and the values for $\mathrm{n}$ and $\mathrm{b}$ were determined to be:

- $n=5.0 \pm 1.5$

$b=0.65_{-0.08}^{+0.10}(\mathrm{GeV} / \mathrm{c})^{-2}$

The $\mathrm{X}_{f}$ distribution for the charm pairs is consistent with the distribution obtained from MC $\Phi_{t}$ correlated generated charm pairs. The mearured value of $\left\langle\mathrm{X}_{f}\right\rangle \mid x_{f}>0$ was consistent with previously measured values done at lower $\sqrt{s}$. This is in contrast to single charm production distributions, which showed a much more central production at 
$\sqrt{s}=38.8 \mathrm{GeV}$ than the distribution observed at $\sqrt{s}=27.4 \mathrm{GeV}$. The $\mathrm{n}$ value measured for the pair $\mathrm{X}_{f}$ distribution is consistent with that obtained from the $\Phi_{t}$ correlated $\mathrm{MC}$, which used single charm produced with the central production distribution measured in the data. The $\mathrm{P}_{t}^{2}$ distribution for the charm pairs was seen to have a tail at high $\left(\mathrm{P}_{t}^{2}>\mathbf{3 . 0}\right.$ $(\mathrm{GeV} / \mathrm{c}))^{2}$, that was not reproduced by the Monte Carlo. The high $\mathrm{P}_{t}^{2}$ tail could be an indication of next to leading order two to three processes in charm production, $g g \rightarrow g q \bar{q}$. This could be confirmed by the presence of hadronic jets recoiling against the charm pair system. The data did not present enough statistics to search for such jets. The $\left\langle\mathrm{P}_{t}^{2}\right\rangle$ was consistent with the measured value from Drell-Yan di-lepton production with 800 $\mathrm{GeV} / \mathrm{c}$ protons, $\left\langle\mathrm{P}_{t}\right\rangle=1.61 \pm 0.16 \mathrm{GeV} / \mathrm{c}[64]$. The $\left\langle\mathrm{P}_{t}^{2}\right\rangle$ from this experiment was compared to the previously measured value of the LEBC-PP experiment as a function of the center of mass energy squared. This comparison was motivated by the Drell-Yan model and allowed the $\left\langle\mathrm{k}_{t}^{2}\right\rangle$ of charm production to be measured [21]:

- $\left\langle\mathrm{k}_{\mathrm{t}}^{2}>\sim 0.70 \pm 0.70(\mathrm{GeV} / \mathrm{c})^{2}\right.$.

This intrinsic $<k_{t}^{2}>$ measurement reflects the transverse momentum of the partons responsible for charm production and the transverse momentum associated with the fragmentation of the charm quarks into mesons. This value is in agreement with the $<k_{t}^{2}>$ used by LEBC in the LUND MC for consistency between the data and the MC. The LUND MC does not reproduce the same slope when the $\left\langle\mathrm{P}_{t}^{2}\right\rangle$ is represented by $\left\langle\mathrm{r}_{\mathrm{t}}\right\rangle=\left\langle\mathrm{k}_{t}^{2}\right\rangle+\mathrm{C} \cdot \mathrm{s}$. 
The charm pair mass distribution has a slightly higher $\langle M(D \bar{D})>$ than the average pair mass measured at lower $\sqrt{s}$. This trend is important for future experiments that hope to explore the perturbative regime of charm production. The mass distribution was seen to fit an exponential distribution:

$$
\frac{d \sigma}{d M} \sim e^{-\alpha M}
$$

with:

- $\mathrm{a}=0.75 \pm 0.15\left(\mathrm{GeV} / \mathrm{c}^{2}\right)^{-1}$

or:

$$
\frac{d \sigma}{d \sqrt{\tau}} \sim e^{-c \sqrt{\tau}}
$$

with:

- $c=29.1 \pm 5.8$.

This mass scaling will allow charm pair experiments at different energies to compare results. The LEBC-PP data is consistent with this result, within the limited statistics. The value is also consistent with that found in di-lepton production in proton-platimum interactions, $c=27.02 \pm 0.16$ at $\sqrt{s}=27.4 \mathrm{GeV}[22]$. The variable $\tau=\frac{M(D D)^{2}}{b}=\mathrm{x}_{a} \mathrm{x}_{b}$ allows one to study the momentum fraction, $x_{a}$ and $x_{b}$, of the partons involved in the reaction. At this level of statistics the gluon distribution appears to be equivalent to that measured for the quarks in di-lepton production.

The azimuthal opening angle $\Phi_{t}$ peaks as expected at $180^{\circ}$. However the $\left\langle\Phi_{t}\right\rangle$ is lower than expected from first order production diagrams (LUND). All three charm pair 
production experiments have measured $\left\langle\Phi_{t}\right\rangle$ to be lower than the LUND MC value. The $\Phi_{t}$ distribution is dependent on the production diagrams and the intrinsic $k_{t}^{2}$ of the partons responsible for charm production. The lower measured $\left\langle\Phi_{t}\right\rangle$ would indicate either a larger intrinsic $<\mathbf{k}_{t}^{2}>$ for the gluons involved in leavy quark production than that used in the LUND MC or contributions from the next to leading order preduction diagrams not installed in the LUND MC.

The statistics limited the strength of the analysis. The observation of charm pair mass and $\left\langle\mathrm{P}_{t}^{2}>\right.$ scaling will aid in future comparisons of charm pair data, if this scaling holds. The analogy to Drell-Yan di-lepton production provides some hope that charm pair plyysics will allow access to the structure functions and intrinsic transverse momentum of the partons (gluons) involved in the charm production process. To do such analysis one would need a large sample of charm pairs, the data from the second run of E653 should have an order of maguitude more charm pairs produced with an incident $\pi$ beam. In . addition the angular distributions center of mass of the charn pair with respect to the target or beam direction can be determined. These angular distributions will provide some insight as to the underlying diagrams responsible for charm production. 


\section{Appendix A}

\section{The Lund Monte Carlo}

The LUND Monte Carlo (MC) is an all purpose high energy physics Monte Carlo simulation. The LUND MC allows the user to define the initial conditions, incident particles, experimental configuration (colliding beams or fixed target) and the reaction of interest (charm production). The LUND MC generates charm via the leading order diagrams and in light of the cross section calculation of Nason, Dawson and Ellis [9] the LUND MC results for charm production are not reliable.

For charm production the user has several switches and initial values that can be change to test their effect on charm production. The important switches are:

- Structure functions of the partons in the incident beam and target. The user can define his/her own structure functions or use one of the seven sets installed in the MC.

- Fragmentation functions used to from mesons and hadrons from the bare quarks. 
- The intrinsic $k_{t}$ distribution and width of the partons.

The LEBC- $\pi$ P and LEBC-PP results were compared to the distributions generated by the LUND MC. The LEBC results agreed best with the LUND MC when the using the EHLQ set 1 structure functions [16], lund fragmentation and an intrinsic $k_{t}^{2}$ parameterized by:

$$
\frac{d N}{d k_{t}^{2}} \sim e^{-\frac{k_{t}^{2}}{0.61(0 . V / c)^{2}}}
$$

The following set of histograms compares the distributions of the 37 charm pairs to those obtained by running the LUND MC in the same configuration used for the LEBC analysis, with the one exception that the intrinsic $\left\langle k_{t}^{2}>\right.$ of the partons was set to $0.70(\mathrm{GeV} / \mathrm{c})^{2}$. Figures 67 through 71 show good agreement between our data for the 37 charm pairs and the distributions from the LUND MC. The one possible difference is the tail in the $P_{t}^{2}$ distribution for the data. LEBC-PP data also was inconsistent with LUND MC at large $P_{t}^{2}$. However with error bars on their plots it hard to determine the statistical significance of their tail. The averages from these distributions were tabulated in Table 24. 


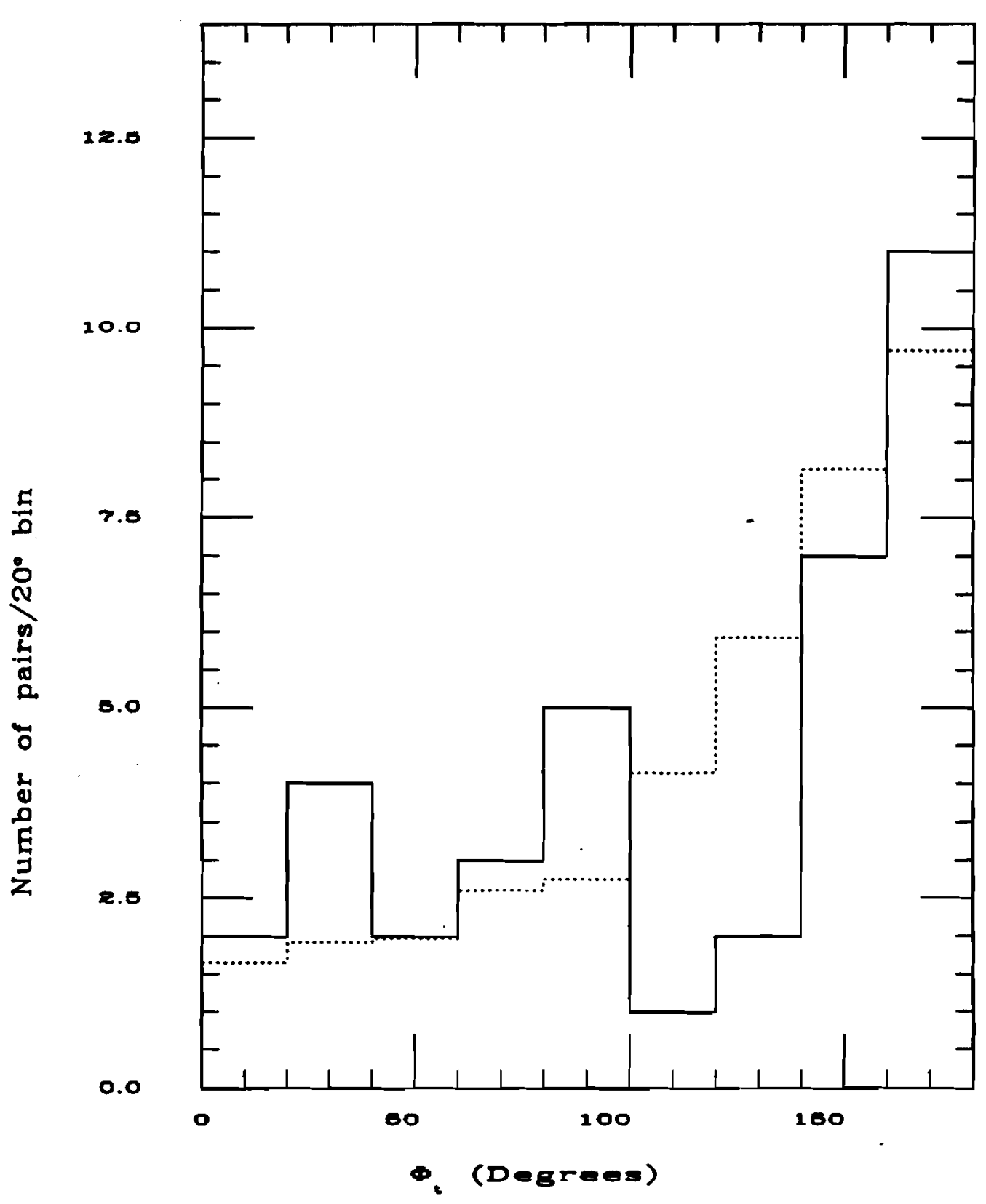

Figure 67: The $\Phi_{t}$ distribution for the 37 charm pairs (solid) and the LUND MC (dots). 


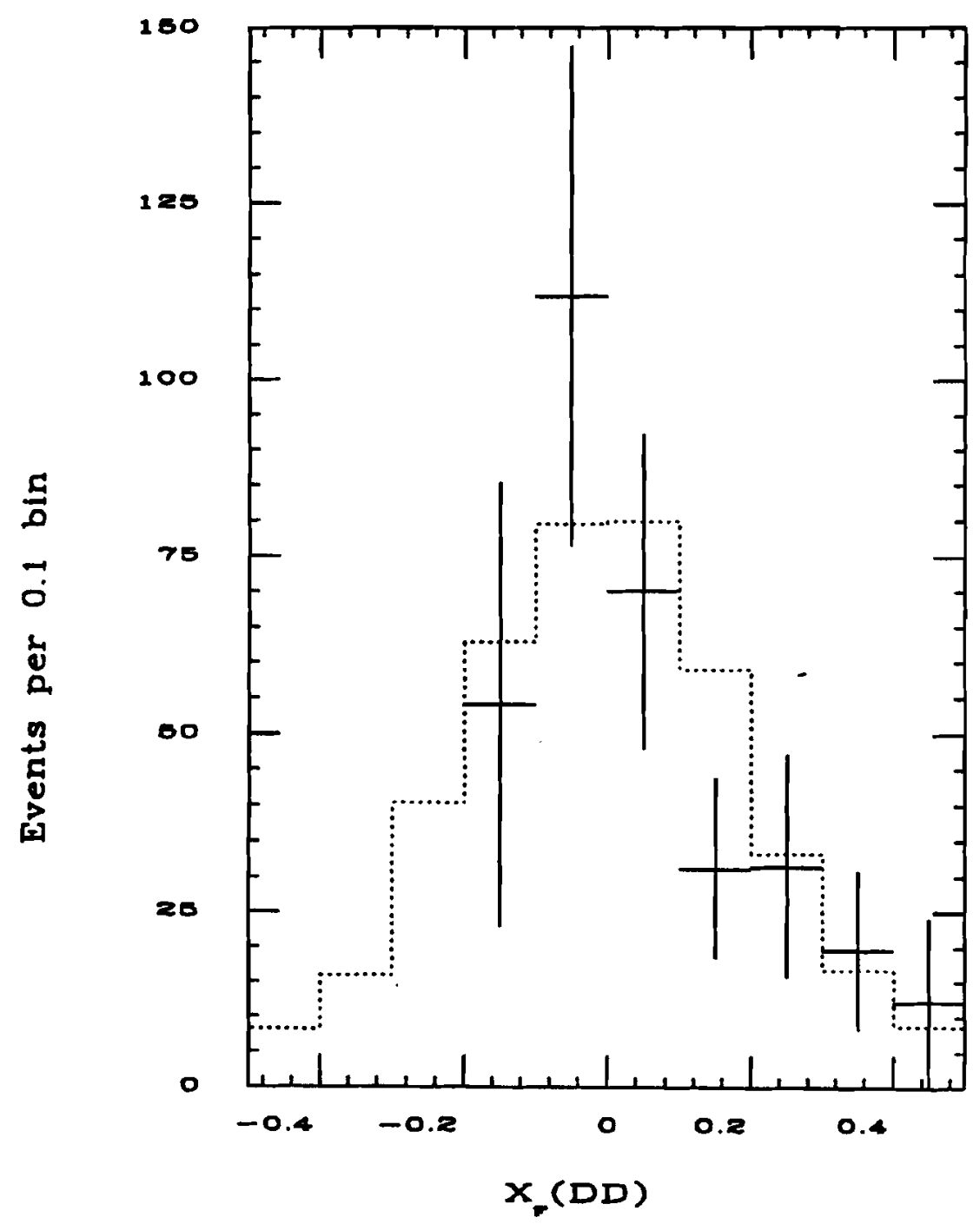

Figure 68: The weighted $\mathrm{X}_{f}$ distribution for the 37 charm pairs (solid) and the LUND MC (dots). 


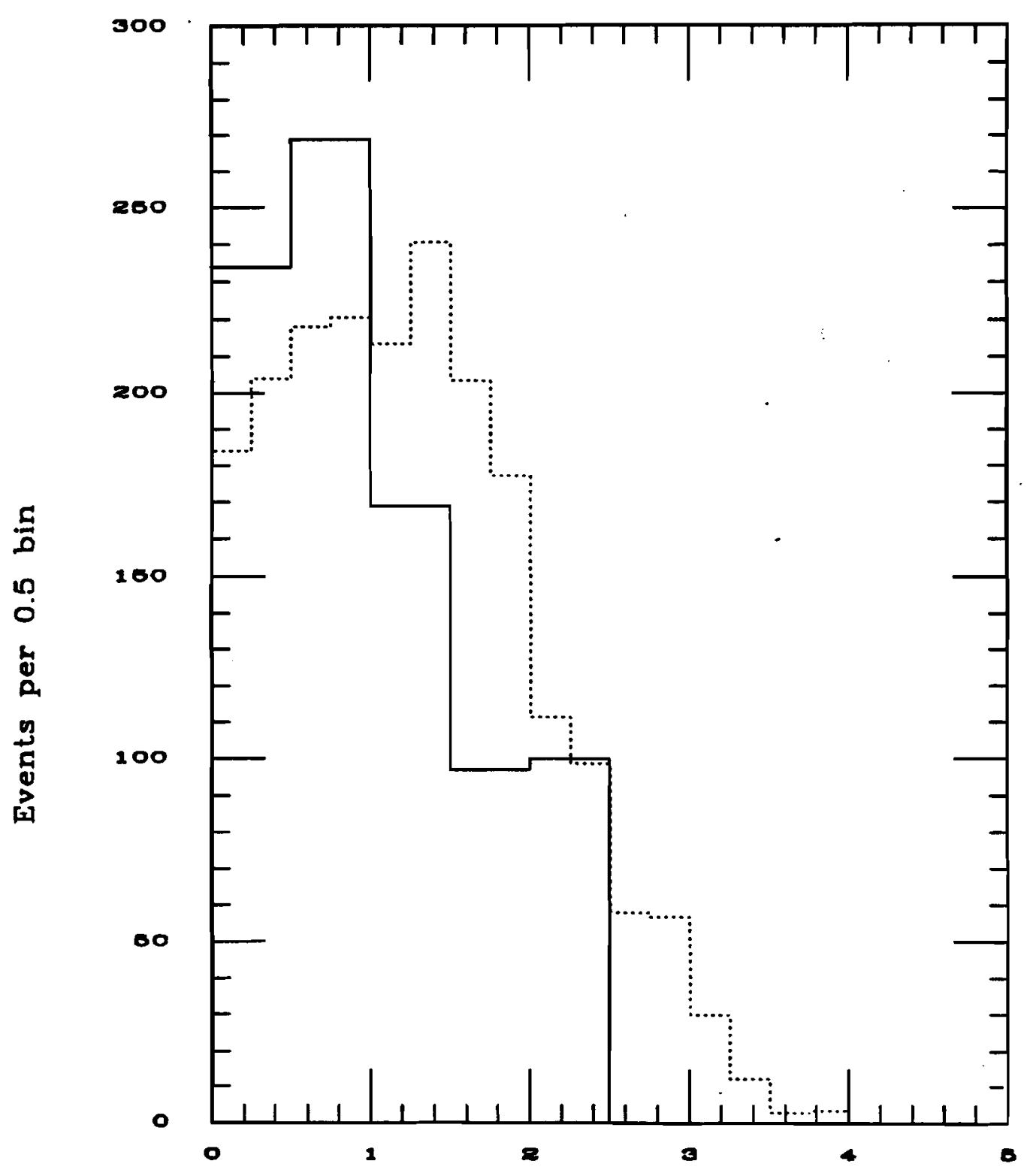

Figure 70: The weiglited rapidity gap distribution for the 37 charm pairs (solid) and that obtained from the LUND MC (dots). 


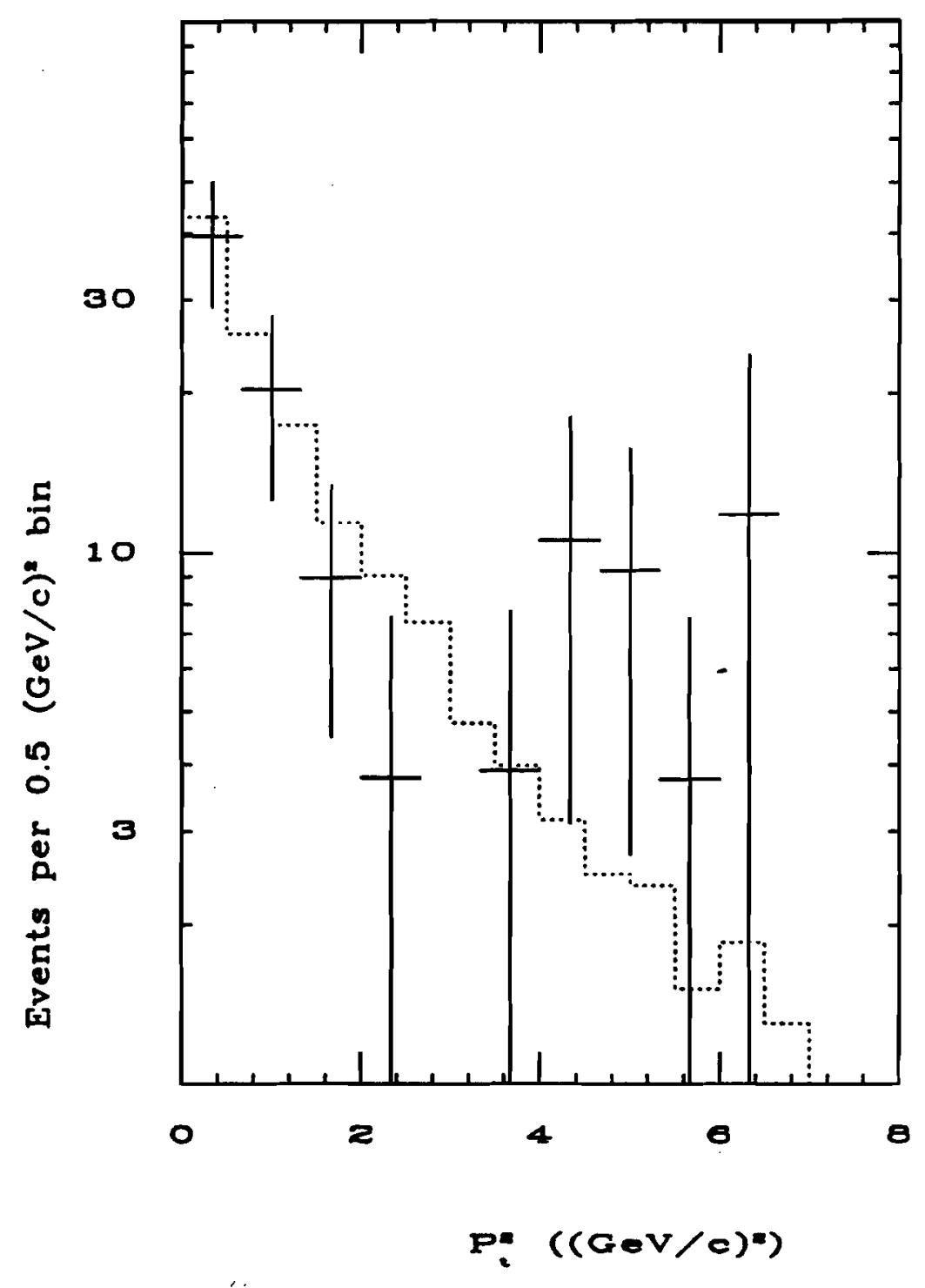

Figure 71: The weighted $P_{t}^{2}$ distribution for the 37 charm pairs (solid) and the LUND MC charm pairs (duts). 


\section{Appendix B}

\section{Event listings}

The kinematic quantities for the 37 charm pair events are listed in the following table.

The table is deciphered as follows: The first row is the accounting row. The first number is the rumning sum of pair events, it starts at one and ends at 37 . The next number is the run number of the event and corresponds to the raw data tape number the event was recorded on. This run number is followed by the event number of the pair.

The next row contains the kinematic information on one of the charm that compose the charm pair. The first number is the decay topology:

- $3 \rightarrow \mathrm{V}-2$

- $4 \rightarrow \mathrm{V}-2 \mu$

- $5 \rightarrow \mathrm{C}-3$

- $6 \rightarrow \mathrm{C}-3 \mu$ 


\section{- $7 \rightarrow V-4$}

The second number is the minimum parent mass of the vertex followed by its error $(\mathrm{GeV} / \mathrm{c2})$. The fourth and fifth numbers are the momentum estimate and "error" for the decay $(\mathrm{GeV} / \mathrm{c})$. The error is listed as a guide and one should remember that it is a non-gaussian error distribution. The sixth number is the $\mathrm{X}_{f}$ of the parent. The seventh number is the $\mathrm{P}_{t}^{2}$ of parent and the eigth number is the rapidity, $y$. The next row contains the same information for the second charm decay in the charm pair. The final row contains the charm pair quantities. The first number is the azimuthal opening angle of the pair, $\Phi_{t}$ (degrees). The pair mass is the second number in the row $\left(\mathrm{GeV} / \mathrm{c}^{2}\right)$. The third number is the charm pair momentum $(\mathrm{GeV} / \mathrm{c})$. The forth is the $\mathrm{X}_{f}$ of the charm pair and the fifth number is $\mathrm{P}_{t}^{2}$ of the charm pair $\left(\mathrm{GeV} / \mathrm{c}^{2}\right)$. The sixth number is the $|\Delta Y|$ of the charm pair. 


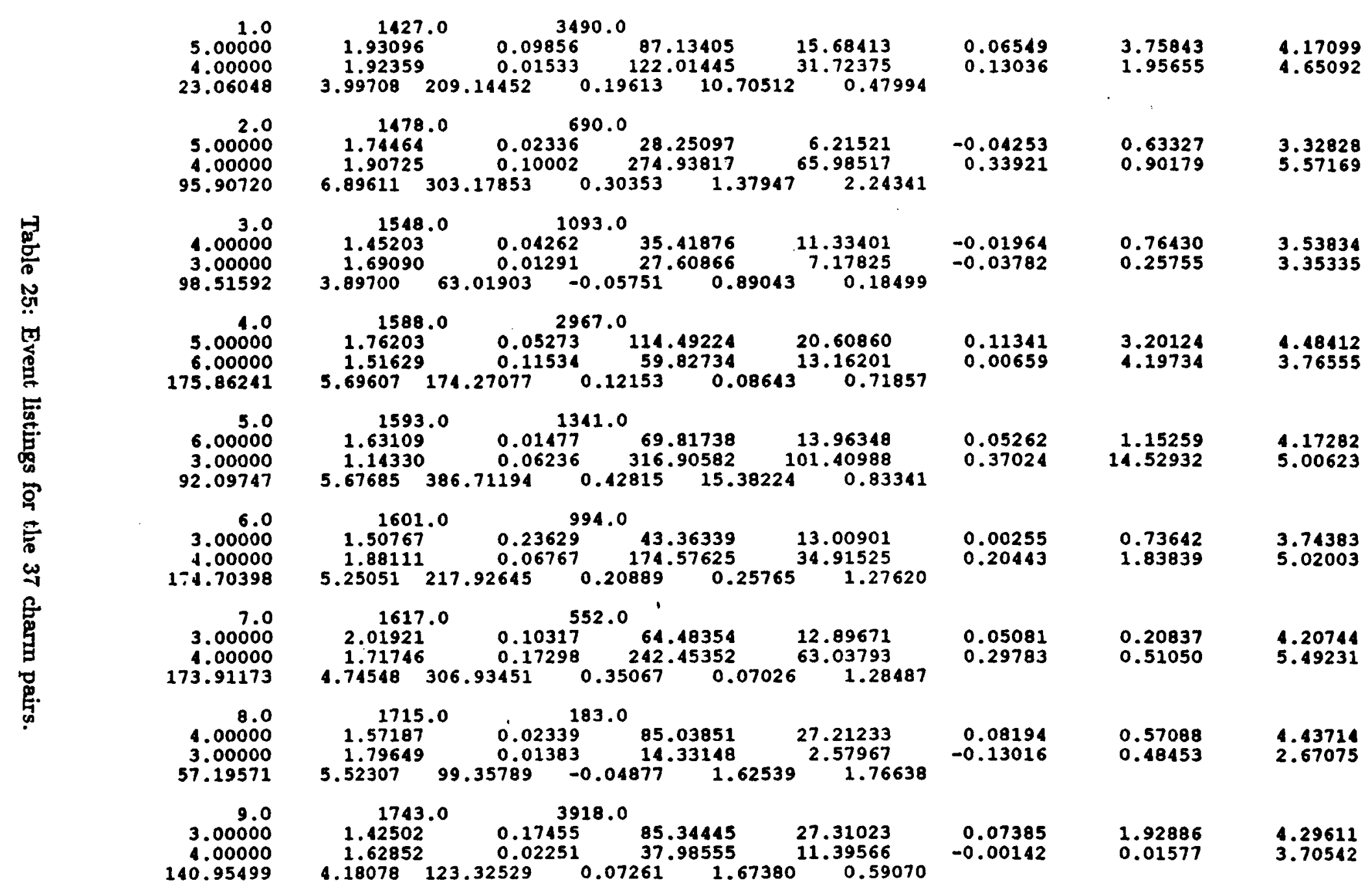




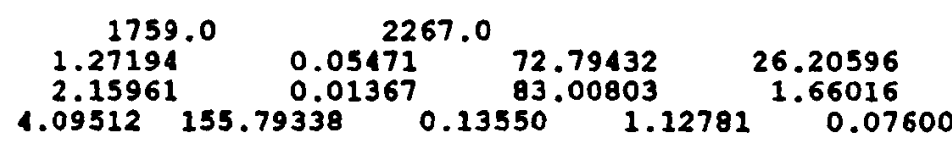

4.98846

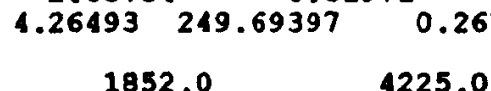

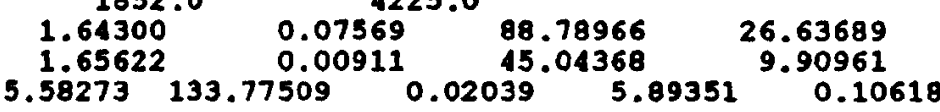

\subsection{7}

11.62501

1.33337

3.82170 $1860.0 \quad 2048.0$

18416

0.17750

40.16079
43.24200

0.10618

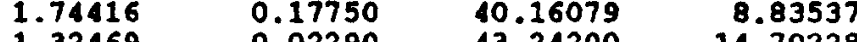

$3.77240 \quad 83.40100 \quad 0.012490 .19080^{14.70228} 0.04755$

0.00380

0.03090

0.22032

3.75888

0.00869

0.22032

3.80643 $1_{1.35189}^{1864.0} \quad 0.24541^{255.0} 1$ 1.35189

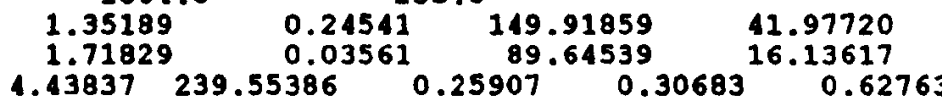

\subsection{2}

0.56509

1.59276

5.00486

4.37723

\section{$2864.0 \quad 2992.0$}

$\begin{array}{lll}54.32148 & 9.77787 & 0.03295\end{array}$

$\begin{array}{llll}1.81510 & 0.02875 & 22.03082 & 3.08432\end{array}$

0.03295
-0.07101

0.13311

0.57511

4.04632

3.08732 
5.61987
0.10212

3.99958

3.90538

\section{$2044.0 \quad 0.04147 .0$}

1.2578600 .00625016 .40056 \begin{tabular}{lllll}
1.74032 & 0.02341 & 106.27325 & 17.00372 & -0.09356 \\
\hline
\end{tabular}

0.30985

3.59192

$2.11479 \quad 3.32616$

$\begin{array}{lllll}2059.0 & { }^{4569.0} & 1 & & \\ 1.75257 & 0.02990 & 61.72466 & 13.57943 & 0.03540\end{array}$

$\begin{array}{llll}1.62288 & 0.02522 & 20.69181 & 5.79371\end{array}$

$-0.14490$

1.41598

3.09731

4.02191 $\begin{array}{lllll}5.64963 & 82.33492 & -0.11086 & 0.80849 & 1.24048\end{array}$

2081.060 .0

1.58747
1.53971

0.20903

66.53959

15.96951

0.05159

0.54937

2.78142

$\begin{array}{llllll}3.92799 & 117.50457 & 0.07727 & 0.17480 & 0.22180\end{array}$

0.02559

0.20530

19454

\section{$2103.0 \quad 0.03889 .0$}

$\begin{array}{llll}1.16193 & 0.03786 & 57.25332 & 20.61119 \\ 1.58068 & 0.02795 & 78.65269 & 23.59581\end{array}$

$\begin{array}{lllll}1.29946 & 135.89049 & 0.09447 & 1.14282 & 0.34538\end{array}$

0.02638

0.06779

1.41949

1.15360

3.94639

4.29178 


\begin{tabular}{|c|}
\hline $\begin{array}{r}28.0 \\
1.00000 \\
3.00000 \\
167.31583\end{array}$ \\
\hline $\begin{array}{r}29.0 \\
3.00000 \\
1.00000 \\
166.87434\end{array}$ \\
\hline $\begin{array}{r}30.0 \\
5.00000 \\
1.00000 \\
10.65471\end{array}$ \\
\hline $\begin{array}{r}31.0 \\
3.00000 \\
1.00000 \\
123.81406\end{array}$ \\
\hline $\begin{array}{r}32.0 \\
5.00000 \\
1.00000 \\
151.46968\end{array}$ \\
\hline $\begin{array}{r}33.0 \\
1.00000 \\
3.00000 \\
20.22398\end{array}$ \\
\hline $\begin{array}{r}34.0 \\
5.00000 \\
1.00000 \\
153.76818\end{array}$ \\
\hline $\begin{array}{r}35.0 \\
6.00000 \\
3.00000 \\
92.62807\end{array}$ \\
\hline $\begin{array}{r}36.0 \\
4.00000 \\
1.00000 \\
174.22079\end{array}$ \\
\hline $\begin{array}{r}37.0 \\
3.00000 \\
6.00000 \\
61.83765\end{array}$ \\
\hline
\end{tabular}

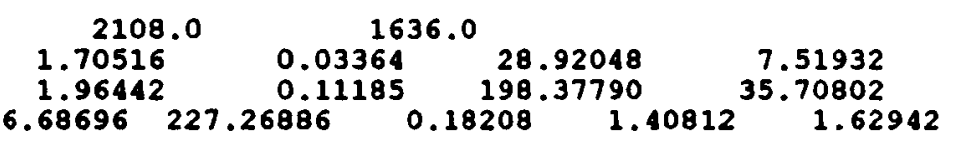

4.43909

0.09310

3.58191

$2250.0 \quad 423.0$

1.80002

0.03440

$15.62780 \quad 2.50045$

$\begin{array}{cccc}1.38666 & 0.03144 & 63.17276 & 20.21528 \\ 4.68678 & 78.79971^{20} & -0.07823 & 4.03560 \\ 1.16356\end{array}$

$-0.10760$

0.23266

.65598

0.02981

2.10044

2.78987 $2250.0 \quad 3759.0$.

1.414750 .01742

$\begin{array}{ll}1.41475 .0 & 0.01742 \\ 31.96967 & 7.67272\end{array}$ $1.90312151 .17607^{1.46139} 0.10597^{0.04696} 0.11769^{35.76508} 1.30906$

$-0.02717$

0.13242

0.54028

0.59216

2282.0 4153.0

$\begin{array}{llll}1.71205 & 0.04597 & 38.94454 & 7.01002\end{array}$

$\begin{array}{llll}1.72791 & 0.04417 & 26.60236 & 6.38457\end{array}$

$-0.01897$

1.45919

3.79272

3.55719

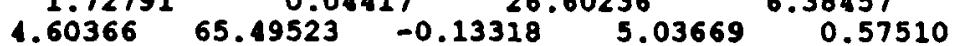

2.98209

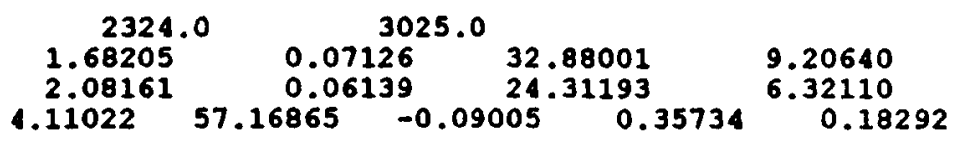

$-0.03690$

31842

3.40254 $\begin{array}{lllll}1.11022 & 57.16865 & -0.09005 & 0.35734 & 0.18292\end{array}$

$-0.05298$

0.30913

3.21962

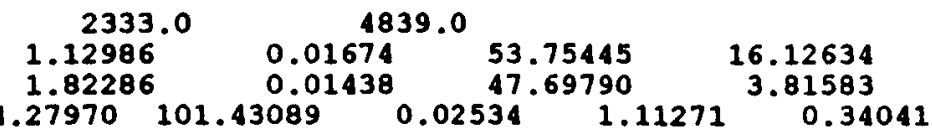

0.03056 $-0.00530$ 
Aw Mama can this really be the end...[65] 


\section{Bibliography}

[1] G. Arnison et.al., Phys. Lett. 122B 103 (1983).

[2] M. Banner et.al., Phys. Lett. 122B 476 (1983).

[3] F. Abe et.al., Phys. Rev. Lett. 63720 (1989).

[4] G.S. Abrams et.al., Phys. Rev. Lett. 63724 (1989).

[5] D.H. Perkins. Introduction to High Energy Physics, Addison-Wesley Publishing Company, pg. 15 (1982).

[6] D. Decamp et.al., Phys. Lett. 235B 399 (1990).

[7] B. Aveda et.al., Phys. Lett. 237B 136 (1990).

[8] F. Alse et.al., Phys. Rev. Lett. 64142 (1990).

[9] P.Nason,S.Dawson and R.K. Ellis., Nuclear Physics B303 607 (1988).

[10] S.J. Brodsky, J.F. Gunion and D.E. Soper, Phys. Rev. D. 36 ?710 (1988)

[11] J.C. Collins, D.E. Soper and G. Sterman, Nuclear Physics B263 37 (1986). 
[12] E.L. Berger, Phys. Rev. D37 1810 (1988).

[13] E.L. Berger, Heavy Flavor Production. Proceedings of the Advanced Workshop on QCD Hard Processes, St. Croix (1988).

[14] E.L. Berger, Heavy Quark Production in Hadron Collisions. Proceedings of the XXII Rencontre de Moriond, Les Arcs, France (1987).

[15] D.W. Duke and J.F. Owens, Phys. Rev. D30 49 (1984).

[16] E. Eichen et. al., Rev. Mod. Phys. 56667 (1984).

[1T] J.P. Rutherford, Hadronic Production of Real and Virual Phons. Proceedings of the 1985 International Symposium on Lepton and Photon Interactions at High Energies, Kyoto, Japan (1985).

[18] S. Faciano et.al., Z. Phys. C 31513 (1986).

[19] S.D. Drell and T.M. Yan, Phys. Rev. Lett. 25316 (1970).

[20] I.R. Kenyon, Rep. Prog. Phys. 451261 (1982).

[21] B. Cox and P.K. Malhotra, Phys. Rev D 2963 (1984).

[22] D.M. Kaplan et. al., Pliys. Rev. Lett. 40435 (1978).

[23] J. Orear, UCRL-8417, Aug. 13 1958. Notes on Statistice for Physicists.

[24] D.B. Gibaut, A Study of the Hadro-Production and Weak Decay of Charm Particles in $360 \mathrm{Gev} / \mathrm{c} \pi^{-}$and $400 \mathrm{GeV} / \mathrm{c} p$ Interactions $\mathrm{Ph} . \mathrm{D}$. Thesis, Oxford University, (1986) 
[25] J. Kirkby, Review of $e^{+} e^{-}$Reactions in the Energy Range of 3 to $9 \mathrm{GeV}$. Proceedings of the 1979 Lepton Photon Conference, Fermilab (1979).

[26] R.M. Edelstein et. al.. The Lead-Liquid Argon Calorimeter for FNAL E653. Proceedings of the IEEE Couference on Nuclear Science, Washington, D.C. Oct. 1988.

[27] N. Stanton et.al., Design and Pèrformance of Large Multisampling Drift. Chambers for Fermilab Experiment 653. Proceedings of the XXIII International Conference on High Energy Physics (1986).

[28] James M. Dunlea, $A$ Study of the $X_{f}$ and $P_{t}$ Distributions in the Production of $D^{ \pm}$ Charmed Mesons. Ph.D. Thesis, Ohio State University (1987).

[29] A. Mokhtarani, Production Characteristics of Neutral and Charged D Mesons in 800 Gev p-Emulsion Interactions Ph.D. Thesis, University of California at Davis (1988).

[30] G. A. Oleynik, Inclusive Charged D Meson Production Cross Section Measurements in 800 GeV p-Emulsion Interactions. Ph.D. Thesis, Ohio State University (1987).

[31] Y-L, Zhang, A Study of $D^{* \pm}$ Hadro-Production and $D^{o}$ Semi-Leptonic Decay. Ph.D. Thesis, Carnegie Mellon University (1989).

[32] T.S. Jaffery, Resistive Plastic, Proportional Tube, Gas Hadron Calorimeter. M.S. Thesis, The University of Oklahoma, (1988).

[33] V. Paolone, Semi-Leptonic Charged D Meson Decays in $800 \mathrm{GeV}$ p-Emulsion Internctions Ph.D. Thesis, University of California at Davis (1990). 
[34] S. Aoki, M.S. Thesis, University of Nagoya (1986).

[35] W. Nichols,Ph.D. Thesis, Carnegie Mellon University (1990)

[36] Kodama et.al., Nucl. Instr. \& Meth. A289 146 (1990)

[37] D.G. Hitlin, CalTedh Pre-print CALT-68-1473 (Nov. 1987) Weak Decays of Chamed Quarks and 5 Leptons.

[38] M. Aguilar-Benitez et. al., Particle Properties Data Booklet (April 1988).

[39] S.P.K. Tavernier, Rep. Prog. Phys. 501439 (198i).

[40] A. Kernan and G.VanDalen, Plyysics Reports 106297 (1984)

[41] P. Weilhammer, CERN-EP/88-08 (Jan. 1988). Review of Recent Experimental Results on Hadroproduction of Heavy Quarks.

[42] M.E. Duffy et. al., Phys. Rev. Lett. 551816 (1985).

[43] H. Cobbaert et. al., Phys. Lett. 191B 456 (1987).

[44] H. Cobbaert et.al., Phys. Lett. 206B 546 (1988).

[45] M.D. Sokoloff et. al., Phys. Rev. Lett. 573003 (1986).

[46] L. Leuking, Proceedings of the $X V^{t h}$ Recontres de Moriond, Les Arcs, France. (1990).

[47] S. Barlag et. al., Z. Phys. C 39451 (1988).

[48] R.P. Feymman, Plyss. Rev. Lett. 231415 (1969). 
[49] R. Anmar et. al. Phys. Rev. Lett. 612185 (1988).

[50] M. Aguilar-Benitez et. al., Physics Letters 189B 476 (1987).

[51] M. Aguilar-Benitez et. al., Z. Phys. C 40321 (1988).

[52] M. Aguilar-Benitez et. al., Physics Letters 164B 404 (1985).

[53] M. Aguilar-Benitez et. al., Physics Letters 161B 404 (1985).

[54] M. Aguilar-Benitez et. al., Z. Phys. C 36559 (1987).

[55] M.E. Duffy et. al., Phys. Rev. Lett. 551816 (1985).

[56] H. Cobbaert et. al., Phys. Lett. 191B 456 (1987).

[57] H. Cobbaert et. al., Phys. Lett. 206B 546 (1988).

[58] M.I. Adamovich et. al. International Symposium on Heavy Quark Physics, Cornell University, Ithaca,N.Y. (1989).

[59] L.Lyons and D.H. Saxon, Oxford pre-print OUNP-89-1 (1988). Techniques for the Study of the Lifetimes of Short-Lived Particles.

[60] J.M. Izen, Slac Pre-print SLAC-PUB-4753 (1988). Semi Leptonic Charm. Decay. Present at the 1988 Meeting of the Divison of Particles and Fields of the American Physical Society, Storrs, Comm.

[61] J.D. Bjorken, Mass of Charm and Strange Baryons (1986) 
[62] K. Kodama et. al. Measurement of the Relative Branching Fraction $\Gamma\left(D^{\circ} \rightarrow K^{\circ} \mu \nu\right) / \Gamma\left(D^{\circ} \rightarrow \mu X\right)$ to be published.

[63] N. Ushida, et. al. E653 proposal (1981)

[64] T. Yoshida et. al., Phys. Rev. D 393516 (1989)

[65] B. Dylan Blonde on Blonde Stuck Inside a Mobile with the Memphis Blues Again (1968). 